\title{
Highly Selective and Switchable Access to Tetrasubstituted Alkenyl Sulfones and Naphthyl Sulfones: 1,4-Aryl Migration versus Cyclization
}

Fei Meng, Honglin Zhang, Jie Li, Jianlin Chun, Yun Shi, Han He, Bin Chen, Zhenbo Gao, and Yingguang Zhu*

Jiangsu Key Laboratory of Pesticide Science and Department of Chemistry, College of Sciences, Nanjing Agricultural University, Nanjing 210095, P. R. China

E-mail: ygzhu@njau.edu.cn

\section{Supporting Information}

\section{Table of Contents}

General information.............................................. S2

Optimization of reaction conditions................................ S2

General procedure for the synthesis of tetrasubstituted alkenyl sulfones........ S3

General procedure for the synthesis of naphthyl sulfones................. S4

Radical-trapping experiments.................................... S4

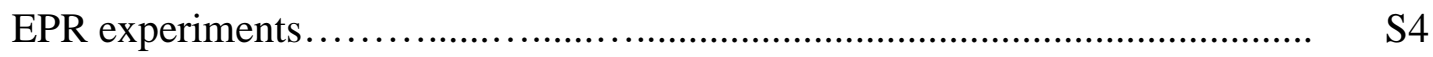

Further demonstration of the reaction pathway to the synthesis of naphthyl

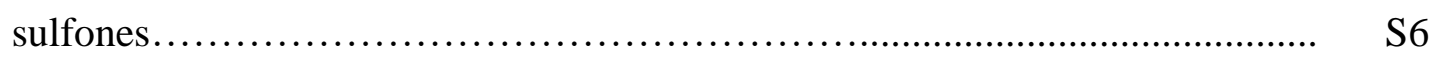

Characterization data................................................... S7

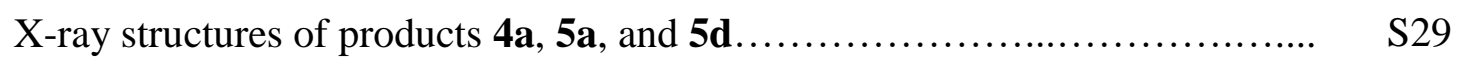

References........................................................ S33

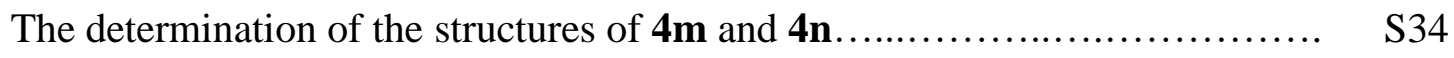

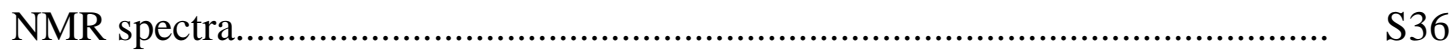


General information. All reagents were purchased from chemical suppliers and used directly without further purification. Column chromatography was carried out on silica gel (200-300 mesh). ${ }^{1} \mathrm{H}$ NMR (400 MHz) and ${ }^{13} \mathrm{C}$ NMR (100 MHz) spectra were recorded on a $400 \mathrm{MHz}$ spectrometer. Chemical shifts $(\delta)$ were reported in ppm, and coupling constants $(J)$ were given in Hertz $(\mathrm{Hz})$. Data were reported as s $=$ singlet, $\mathrm{d}=$ doublet, $\mathrm{t}=$ triplet, $\mathrm{q}=$ quartet, $\mathrm{dd}=$ doublet of doublets, $\mathrm{td}=$ triplet of doublets, ddd $=$ doublet of double doublets, $\mathrm{m}=$ multiplet. High-resolution mass spectra (HRMS) were recorded on a Micromass CI-TOF mass spectrometer. Melting points were uncorrected. Homopropargylic alcohol substrates $\mathbf{1 a - 1 n}, \mathbf{1 p}, \mathbf{2 a - 2 o}, \mathbf{2 q}$, and $\mathbf{2 s -}$ $\mathbf{2 v}$ were prepared according to the literature procedure. ${ }^{1}$ Substrates $\mathbf{1 0}$ and $\mathbf{2 p}$ were synthesized following the literature methods. ${ }^{1,2}$ Substrates $\mathbf{1 q}$ and $\mathbf{2 r}$ were prepared following the literature methods. ${ }^{3,4}$ Sodium $p$-toluenethiosulfonate was obtained according to the reported method. ${ }^{5}$

Table S1. Optimization of Reaction Conditions ${ }^{a}$

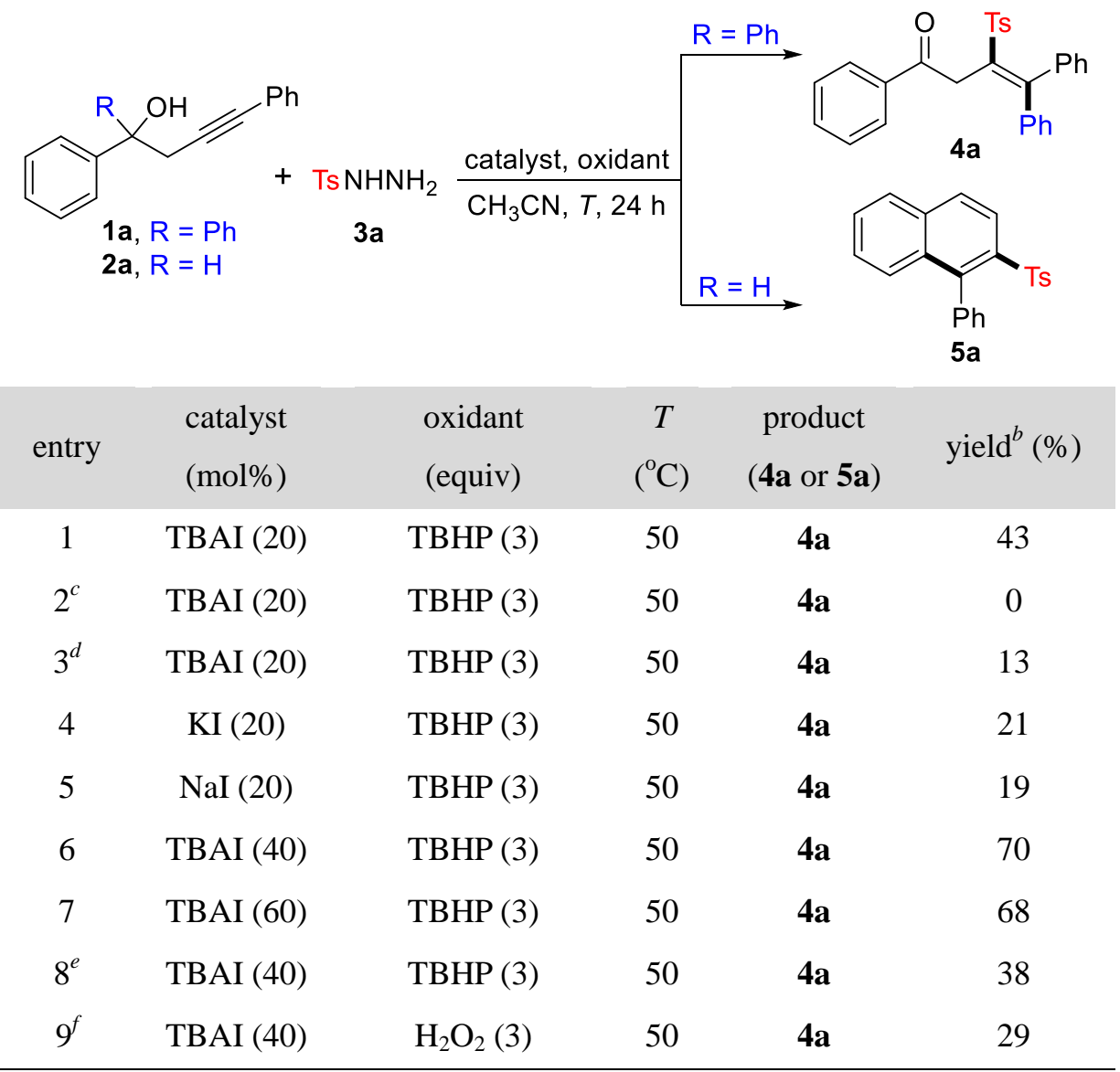




\begin{tabular}{|c|c|c|c|c|c|}
\hline 10 & TBAI (40) & ТВРВ (3) & 50 & $4 a$ & 35 \\
\hline 11 & TBAI (40) & DTBP (3) & 50 & $4 a$ & 16 \\
\hline 12 & TBAI (40) & TBHP (5) & 50 & $4 a$ & 85 \\
\hline 13 & TBAI (40) & TBHP (5) & 80 & $4 a$ & 15 \\
\hline 14 & TBAI (40) & TBHP (5) & 80 & $5 \mathbf{a}$ & 65 \\
\hline $15^{g}$ & TBAI (40) & TBHP (5) & 80 & $5 a$ & 0 \\
\hline $16^{h}$ & TBAI (40) & TBHP (5) & 80 & $5 \mathbf{a}$ & trace \\
\hline 17 & TBAI (20) & TBHP (5) & 80 & $5 \mathbf{a}$ & 76 \\
\hline 18 & TBAI (60) & TBHP (5) & 80 & $5 \mathbf{a}$ & 44 \\
\hline $19^{e}$ & TBAI (20) & TBHP (5) & 80 & $5 a$ & 53 \\
\hline $20^{f}$ & TBAI (20) & $\mathrm{H}_{2} \mathrm{O}_{2}(5)$ & 80 & $5 \mathbf{a}$ & 48 \\
\hline 21 & TBAI (20) & ТВPB (5) & 80 & $5 \mathbf{a}$ & 49 \\
\hline 22 & TBAI (20) & DTBP (5) & 80 & $5 \mathbf{a}$ & trace \\
\hline 23 & TBAI (20) & TBHP (5) & 100 & $5 \mathbf{a}$ & 53 \\
\hline 24 & TBAI (20) & TBHP (5) & 50 & $5 a$ & 29 \\
\hline
\end{tabular}

${ }^{a}$ All reactions were performed with homopropargylic alcohol 1a or $\mathbf{2 a}(0.20 \mathrm{mmol}), \mathrm{TsNHNH}_{2}$, catalyst, and oxidant in $\mathrm{CH}_{3} \mathrm{CN}(2.0 \mathrm{~mL})$ for $24 \mathrm{~h}$ unless otherwise stated. For entry 1 and entries 4-13, $\mathrm{TsNHNH}_{2}$ (3 equiv) was used. For entry 14 and entries $17-24, \mathrm{TsNHNH}_{2}$ (5 equiv) was used. For entries 1-7, entries $12-18$ and entries $23-24$, TBHP (5.5 $\mathrm{M}$ in decane) was used. ${ }^{b}$ Isolated yields based on 1a or $\mathbf{2 a} .{ }^{c}$ Sodium $p$-toluenesulfinate ( 3 equiv) was used. ${ }^{d}$ Sodium $p$-toluenethiosulfonate (3 equiv) was used. ${ }^{e}$ TBHP (70\% in water). ${ }^{f} \mathrm{H}_{2} \mathrm{O}_{2}$ (30\% in water). ${ }^{g}$ Sodium $p$-toluenesulfinate (5 equiv) was used. ${ }^{h}$ Sodium $p$-toluenethiosulfonate (5 equiv) was used. $\mathrm{TsNHNH}_{2}=p$-toluenesulfonyl hydrazide, TBAI $=$ tetra- $n$-butylammonium iodide, TBHP $=$ tert-butyl hydroperoxide, TBPB = tert-butyl peroxybenzoate, $\mathrm{DTBP}=$ di-tert-butylperoxide .

General procedure for the synthesis of tetrasubstituted alkenyl sulfones 4 via sulfonylation/1,4-aryl migration (Scheme 2). To a reaction tube equipped with a magnetic stir bar were added homopropargylic alcohol 1 (0.20 mmol), sulfonyl hydrazide 3 (0.60 mmol), TBAI (29.5 mg, $0.080 \mathrm{mmol})$, TBHP (5.5 M in decane, 182 $\mu \mathrm{L}, 1.0 \mathrm{mmol})$, and $\mathrm{CH}_{3} \mathrm{CN}(2 \mathrm{~mL})$. The reaction mixture was vigorously stirred at 50 ${ }^{\circ} \mathrm{C}$ under nitrogen atmosphere for $24 \mathrm{~h}$ and concentrated under reduced pressure. The residue was purified by column chromatography on silica gel (eluent: petroleum ether/ethyl acetate $=5: 1)$ to give product 4 . 
General procedure for the synthesis of naphthyl sulfones 5 via sulfonylation/cyclization/dehydration (Scheme 3). To a reaction tube equipped with a magnetic stir bar were added homopropargylic alcohol $2(0.20 \mathrm{mmol})$, sulfonyl hydrazide 3 (1.0 mmol), TBAI (14.8 mg, $0.040 \mathrm{mmol})$, TBHP (182 $\mu \mathrm{L}, 1.0 \mathrm{mmol})$, and $\mathrm{CH}_{3} \mathrm{CN}(2 \mathrm{~mL})$. The reaction mixture was stirred at $80{ }^{\circ} \mathrm{C}$ under $\mathrm{N}_{2}$ for $24 \mathrm{~h}$ and concentrated under reduced pressure. The residue was purified by column chromatography on silica gel (eluent: petroleum ether/ethyl acetate $=20: 1$ ) to afford product 5 .

\section{The experiment of the addition of radical scavenger TEMPO (Schemes $4 \mathrm{~b}$ and} 4c). To a reaction tube equipped with a magnetic stir bar were added homopropargylic alcohol 1a $(59.7 \mathrm{mg}, 0.20 \mathrm{mmol})$ or $\mathbf{2 a}(44.5 \mathrm{mg}, 0.20 \mathrm{mmol})$, $\mathrm{TsNHNH}_{2}(111.7 \mathrm{mg}, 0.60 \mathrm{mmol}$ or $186.2 \mathrm{mg}, 1.0 \mathrm{mmol})$, TBAI $(29.5 \mathrm{mg}, 0.080$ mmol or $14.8 \mathrm{mg}, 0.040 \mathrm{mmol})$, TBHP (182 $\mu \mathrm{L}, 1.0 \mathrm{mmol})$, TEMPO (93.7 mg, 0.60 mmol), and $\mathrm{CH}_{3} \mathrm{CN}(2 \mathrm{~mL})$. The reaction mixture was stirred at $50{ }^{\circ} \mathrm{C}$ or $80{ }^{\circ} \mathrm{C}$ under $\mathrm{N}_{2}$ for $24 \mathrm{~h}$. Thin-layer chromatography (TLC) analysis indicated that the formation of the product $\mathbf{4 a}$ or $\mathbf{5 a}$ was not observed.

\section{Electron paramagnetic resonance (EPR) experiments}

In order to further investigate whether or not the radical species are involved in the switchable reaction processes, electron paramagnetic resonance (EPR) experiments were carried out using 5,5-dimethyl-1-pyrroline $N$-oxide (DMPO) as the radical spin trapping agent. For the sulfonylation/1,4-aryl migration reaction, when DMPO was added under standard reaction conditions in the absence of $\mathrm{TsNHNH}_{2}$, an EPR signal was observed (Figure S1-A), indicating that tert-butoxyl radical $^{6}$ could be generated. When DMPO was added under standard reaction conditions, a different EPR signal was detected, suggesting that a new radical species was formed (Figure S1-B). For the sulfonylation/cyclization/dehydration reaction, EPR experiments (with or without $\mathrm{TsNHNH}_{2}$ ) gave the similar results (Figure S2). The EPR studies and the above radical-trapping experiments with TEMPO suggested that the switchable 
reaction processes might proceed via a radical pathway.

(A) $1 \mathrm{a}+\mathrm{TBAI}+\mathrm{TBHP}+\mathrm{DMPO}$

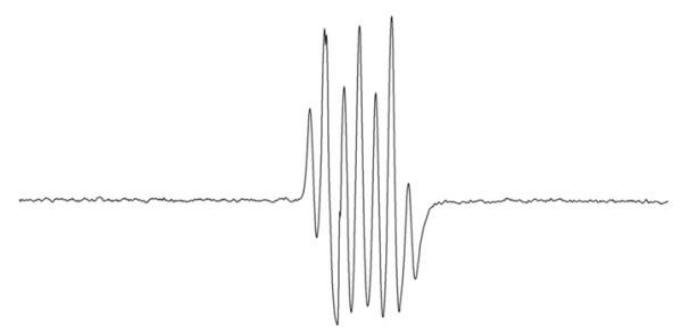

(B) $1 \mathrm{a}+\mathrm{TBAI}+\mathrm{TBHP}+\mathrm{TsNHNH}_{2}+\mathrm{DMPO}$

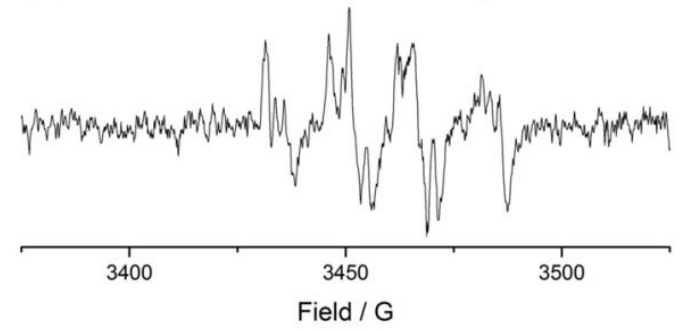

Figure S1. The EPR spectra ( $\mathrm{X}$ band, $10.5 \mathrm{GHz}$, room temperature) of the reaction mixture. (A) 1a, TBAI, TBHP, and DMPO in $\mathrm{CH}_{3} \mathrm{CN}$ at $50{ }^{\circ} \mathrm{C}$; (B) 1a, TBAI, TBHP, $\mathrm{TsNHNH}_{2}$, and DMPO in $\mathrm{CH}_{3} \mathrm{CN}$ at $50{ }^{\circ} \mathrm{C}$.

(A) $2 a+T B A I+T B H P+D M P O$

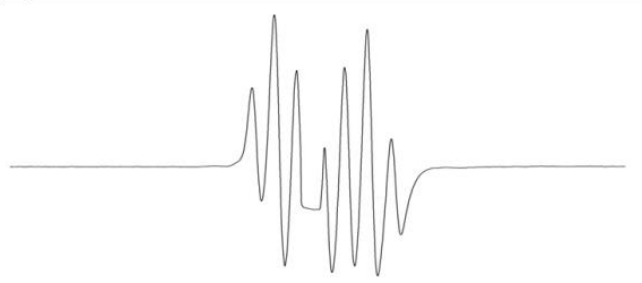

(B) $2 \mathrm{a}+\mathrm{TBAI}+\mathrm{TBHP}+\mathrm{TsNHNH}_{2}+\mathrm{DMPO}$

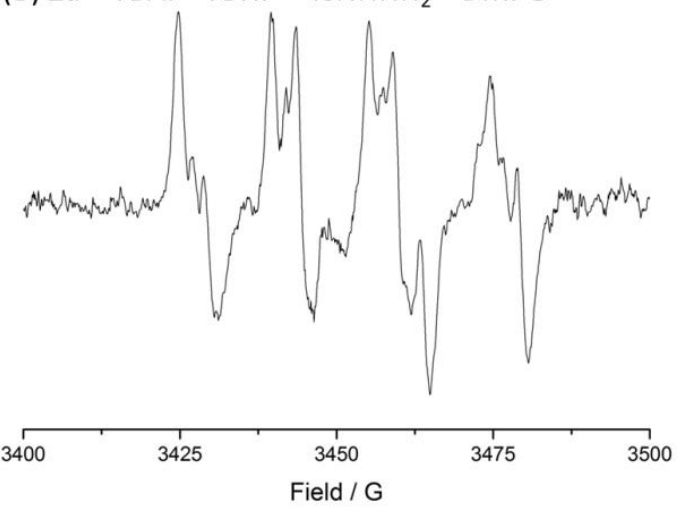

Figure S2. The EPR spectra ( $\mathrm{X}$ band, $10.5 \mathrm{GHz}$, room temperature) of the reaction mixture. (A) 2a, TBAI, TBHP, and DMPO in $\mathrm{CH}_{3} \mathrm{CN}$ at $80{ }^{\circ} \mathrm{C}$; (B) 2aa, TBAI, TBHP, TsNHNH 2 , and DMPO in $\mathrm{CH}_{3} \mathrm{CN}$ at $80{ }^{\circ} \mathrm{C}$. 


\section{The procedure for the EPR experiments of sulfonylation/1,4-aryl migration}

Figure S1-A: To a reaction tube equipped with a magnetic stir bar were added homopropargylic alcohol 1a $(0.050 \mathrm{mmol})$, TBAI $(7.38 \mathrm{mg}, 0.020 \mathrm{mmol})$, TBHP (45.5 $\mu \mathrm{L}, 0.25 \mathrm{mmol})$, DMPO (17.0 mg, $0.15 \mathrm{mmol})$ and $\mathrm{CH}_{3} \mathrm{CN}(0.25 \mathrm{~mL})$. The reaction mixture was stirred at $50{ }^{\circ} \mathrm{C}$ under nitrogen atmosphere for $2 \mathrm{~min}$. Then the reaction mixture was immediately subjected to EPR analysis on a Bruker microESR spectrometer ( $\mathrm{X}$ band, $10.5 \mathrm{GHz}$, room temperature).

Figure S1-B: To a reaction tube equipped with a magnetic stir bar were added $1 \mathrm{a}(0.050 \mathrm{mmol}), \mathrm{TsNHNH}_{2}(27.9 \mathrm{mg}, 0.15 \mathrm{mmol}), \mathrm{TBAI}(7.38 \mathrm{mg}, 0.020 \mathrm{mmol})$, TBHP (45.5 $\mu \mathrm{L}, 0.25 \mathrm{mmol})$, DMPO (17.0 mg, $0.15 \mathrm{mmol}$ ), and $\mathrm{CH}_{3} \mathrm{CN}(0.25 \mathrm{~mL})$. The reaction mixture was stirred at $50{ }^{\circ} \mathrm{C}$ under $\mathrm{N}_{2}$ for $2 \mathrm{~min}$. The reaction mixture was immediately subjected to EPR analysis ( $\mathrm{X}$ band, $10.5 \mathrm{GHz}$, room temperature).

\section{The procedure for the EPR experiments of sulfonylation/cyclization/dehydration}

Figure S2-A: To a reaction tube equipped with a magnetic stir bar were added homopropargylic alcohol 2a $(0.050 \mathrm{mmol})$, TBAI $(3.69 \mathrm{mg}, 0.010 \mathrm{mmol})$, TBHP (45.5 $\mu \mathrm{L}, 0.25 \mathrm{mmol})$, DMPO (17.0 mg, $0.15 \mathrm{mmol})$, and $\mathrm{CH}_{3} \mathrm{CN}(0.25 \mathrm{~mL})$. The reaction mixture was stirred at $80{ }^{\circ} \mathrm{C}$ under nitrogen atmosphere for $30 \mathrm{~s}$. Then the reaction mixture was immediately subjected to EPR analysis (X band, $10.5 \mathrm{GHz}$, room temperature).

Figure S2-A: To a reaction tube equipped with a magnetic stir bar were added 2a $(0.050 \mathrm{mmol}), \mathrm{TsNHNH}_{2}(46.6 \mathrm{mg}, 0.25 \mathrm{mmol}), \mathrm{TBAI}(3.69 \mathrm{mg}, 0.010 \mathrm{mmol})$, TBHP (45.5 $\mu \mathrm{L}, 0.25 \mathrm{mmol})$, DMPO (17.0 mg, $0.15 \mathrm{mmol}$ ), and $\mathrm{CH}_{3} \mathrm{CN}(0.25 \mathrm{~mL})$. The reaction mixture was stirred at $80{ }^{\circ} \mathrm{C}$ under $\mathrm{N}_{2}$ for $2 \mathrm{~min}$. The reaction mixture was immediately subjected to EPR analysis ( $\mathrm{X}$ band, $10.5 \mathrm{GHz}$, room temperature).

\section{Further demonstration of the reaction pathway to the synthesis of naphthyl} sulfones. It was proposed that the reaction of homopropargylic alcohols with sulfonyl hydrazides for the synthesis of naphthyl sulfones proceeds through sulfonyl radical addition, 5-ipso cyclization, 1,2-migration, oxidation, and dehydration 
(Scheme S1, path a), which is supported by the results that the single regioisomeric product 5d (confirmed by single-crystal X-ray analysis) was obtained from the para-chlorophenyl-substituted homopropargylic alcohol $\mathbf{2 d}$. The formation of the other regioisomer $\mathbf{5} \mathbf{d}^{\prime}$ was not observed in this reaction, thus suggesting that the possible reaction pathway, involving sulfonyl radical addition, 6-endo-trig cyclization, oxidation, and dehydration (Scheme S1, path b), can be ruled out.

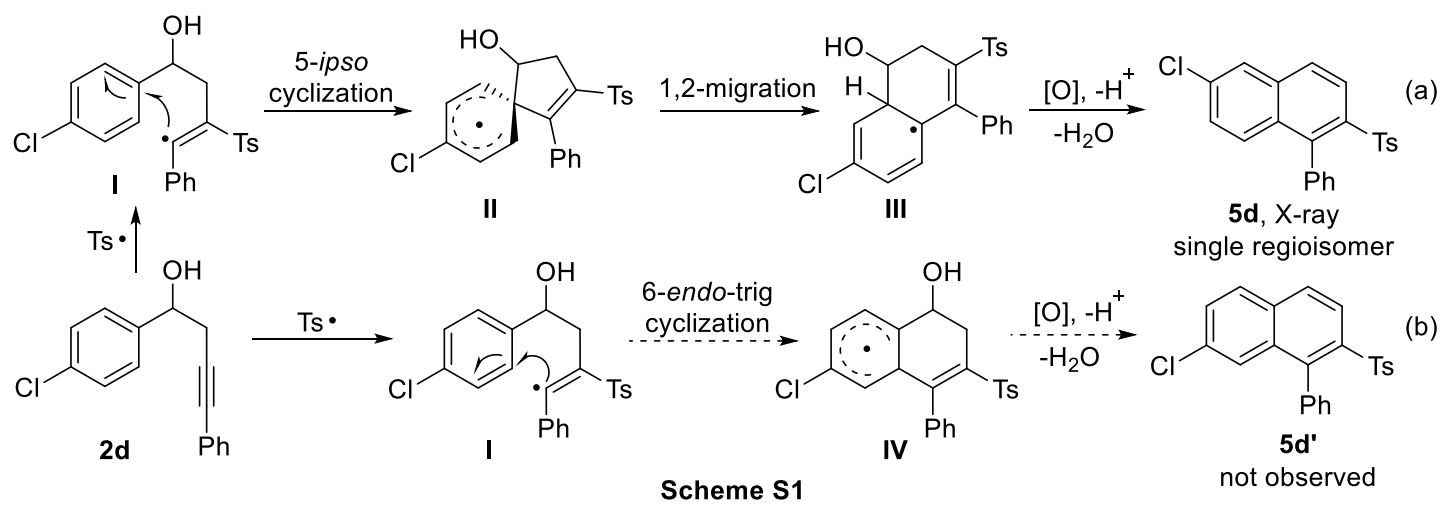

\section{Characterization data}<smiles>CC(CC(=O)c1ccccc1)=C(c1ccccc1)c1ccccc1</smiles>

White solid (77.1 mg, 85\%); mp 142-144 ${ }^{\circ} \mathrm{C} ;{ }^{1} \mathrm{H}$ NMR (400 MHz, $\left.\mathrm{CDCl}_{3}\right) \delta 8.03-$ $7.98(\mathrm{~m}, 2 \mathrm{H}), 7.60-7.55(\mathrm{~m}, 1 \mathrm{H}), 7.47$ (t, $J=7.6 \mathrm{~Hz}, 2 \mathrm{H}), 7.42(\mathrm{~d}, J=8.3 \mathrm{~Hz}, 2 \mathrm{H})$, $7.21(\mathrm{~s}, 5 \mathrm{H}), 7.17-7.12(\mathrm{~m}, 1 \mathrm{H}), 7.05(\mathrm{t}, J=7.6 \mathrm{~Hz}, 2 \mathrm{H}), 7.01-6.93(\mathrm{~m}, 4 \mathrm{H}), 4.33$ (s, 2H), 2.31 (s, 3H); $\left.{ }^{13} \mathrm{C} \mathrm{NMR} \mathrm{(100} \mathrm{MHz,} \mathrm{CDCl}_{3}\right) \delta 197.4,154.6,142.9,140.6,138.3$, $137.5,136.6,133.3,129.5,128.7,128.6,128.53,128.49,128.3,128.1,127.7,127.5$, 127.4, 40.8, 21.5; HRMS (CI-TOF) m/z: $[\mathrm{M}+\mathrm{H}]^{+}$calcd for $\mathrm{C}_{29} \mathrm{H}_{25} \mathrm{O}_{3} \mathrm{~S}$ 453.1519, found 453.1530 . 


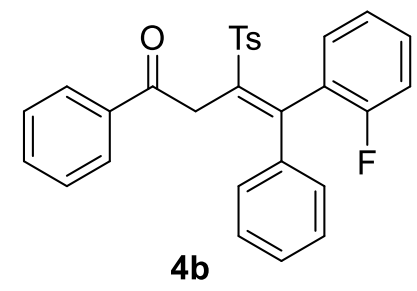

White solid (71.3 mg, 76\%); mp 123-125 ${ }^{\circ} \mathrm{C} ;{ }^{1} \mathrm{H}$ NMR $\left(400 \mathrm{MHz}, \mathrm{CDCl}_{3}\right) \delta 7.99(\mathrm{~d}, J$ $=7.5 \mathrm{~Hz}, 2 \mathrm{H}), 7.57(\mathrm{t}, J=7.3 \mathrm{~Hz}, 1 \mathrm{H}), 7.52-7.43(\mathrm{~m}, 4 \mathrm{H}), 7.37(\mathrm{t}, J=6.9 \mathrm{~Hz}, 1 \mathrm{H})$, $7.23(\mathrm{dd}, J=12.9,4.3 \mathrm{~Hz}, 5 \mathrm{H}), 7.18-7.12(\mathrm{~m}, 1 \mathrm{H}), 7.10-7.00(\mathrm{~m}, 3 \mathrm{H}), 6.56(\mathrm{t}, J=$

$8.9 \mathrm{~Hz}, 1 \mathrm{H}), 4.60(\mathrm{~d}, J=17.6 \mathrm{~Hz}, 1 \mathrm{H}), 4.08(\mathrm{~d}, J=17.6 \mathrm{~Hz}, 1 \mathrm{H}), 2.35(\mathrm{~s}, 3 \mathrm{H}) ;{ }^{13} \mathrm{C}$ NMR (100 MHz, $\left.\mathrm{CDCl}_{3}\right) \delta 196.7,158.6(\mathrm{~d}, J=247.8 \mathrm{~Hz}), 148.7,143.3,140.4,139.0$, 137.0, 136.4, 133.3, 131.7 (d, $J=2.6 \mathrm{~Hz}), 130.3$ (d, $J=8 \mathrm{~Hz}), 128.8,128.62,128.58$, 128.4, 128.2, 127.9, 127.3, 126.1 (d, $J=15.6 \mathrm{~Hz}), 123.3$ (d, $J=3.4 \mathrm{~Hz}), 115.0$ (d, $J=$ $2.1 \mathrm{~Hz}$ ), 40.4, 21.5; HRMS (CI-TOF) m/z: $[\mathrm{M}+\mathrm{H}]^{+}$calcd for $\mathrm{C}_{29} \mathrm{H}_{24} \mathrm{FO}_{3} \mathrm{~S} 471.1425$, found 471.1425 .<smiles>O=C(C/C([13I])=C(/c1ccccc1)c1cccc(F)c1)c1ccccc1</smiles>

White solid (73.5 mg, 78\%); mp 145-146 ${ }^{\circ} \mathrm{C} ;{ }^{1} \mathrm{H}$ NMR $\left(400 \mathrm{MHz}, \mathrm{CDCl}_{3}\right) \delta 7.99(\mathrm{~d}, J$ $=7.4 \mathrm{~Hz}, 2 \mathrm{H}), 7.56(\mathrm{t}, J=7.3 \mathrm{~Hz}, 1 \mathrm{H}), 7.47(\mathrm{dd}, J=14.8,7.7 \mathrm{~Hz}, 4 \mathrm{H}), 7.21(\mathrm{~s}, 5 \mathrm{H})$, $7.09(\mathrm{dd}, J=13.9,7.9 \mathrm{~Hz}, 1 \mathrm{H}), 7.04(\mathrm{~d}, J=8.1 \mathrm{~Hz}, 2 \mathrm{H}), 6.93(\mathrm{~d}, J=7.6 \mathrm{~Hz}, 1 \mathrm{H})$, $6.84(\mathrm{td}, J=8.4,1.8 \mathrm{~Hz}, 1 \mathrm{H}), 6.45(\mathrm{~d}, J=9.4 \mathrm{~Hz}, 1 \mathrm{H}), 4.32(\mathrm{~s}, 2 \mathrm{H}), 2.33(\mathrm{~s}, 3 \mathrm{H}) ;{ }^{13} \mathrm{C}$ NMR $\left(100 \mathrm{MHz}, \mathrm{CDCl}_{3}\right) \delta 197.0,162.0(\mathrm{~d}, J=245.4 \mathrm{~Hz}), 152.7(\mathrm{~d}, J=1.8 \mathrm{~Hz})$, 143.4, 140.1 (d, $J=7.8 \mathrm{~Hz}), 139.8,139.2,137.2,136.4,133.3,129.0$ (d, $J=8.2 \mathrm{~Hz})$, 128.7, 128.6, 128.2, 128.0, 127.2, $125.4(\mathrm{~d}, J=2.8 \mathrm{~Hz}), 116.1,115.9,114.8,114.6$, 40.7, 21.4; HRMS (CI-TOF) m/z: $[\mathrm{M}+\mathrm{H}]^{+}$calcd for $\mathrm{C}_{29} \mathrm{H}_{24} \mathrm{FO}_{3} \mathrm{~S} 471.1425$, found 471.1444 . 
<smiles>CC(CC(=O)c1ccccc1)=C(c1ccccc1)c1ccc(Cl)cc1</smiles>

White solid (82.5 mg, 85\%); mp 142-144 ${ }^{\circ} \mathrm{C} ;{ }^{1} \mathrm{H}$ NMR (400 MHz, $\left.\mathrm{CDCl}_{3}\right) \delta 8.03-$ $7.97(\mathrm{~m}, 2 \mathrm{H}), 7.61-7.55(\mathrm{~m}, 1 \mathrm{H}), 7.48(\mathrm{t}, J=7.4 \mathrm{~Hz}, 4 \mathrm{H}), 7.25-7.16(\mathrm{~m}, 5 \mathrm{H}), 7.04$ $(\mathrm{dd}, J=12.3,8.4 \mathrm{~Hz}, 4 \mathrm{H}), 6.89(\mathrm{~d}, J=8.3 \mathrm{~Hz}, 2 \mathrm{H}), 4.32(\mathrm{~s}, 2 \mathrm{H}), 2.35(\mathrm{~s}, 3 \mathrm{H}) ;{ }^{13} \mathrm{C}$ NMR $\left(100 \mathrm{MHz}, \mathrm{CDCl}_{3}\right) \delta 197.2,153.0,143.4,140.0,139.2,137.4,136.7,136.4$, $134.0,133.3,130.9,128.8,128.7,128.62$, 128.60, 128.3, 128.1, 127.7, 127.4, 40.8, 21.5; HRMS (CI-TOF) m/z: $[\mathrm{M}+\mathrm{H}]^{+}$calcd for $\mathrm{C}_{29} \mathrm{H}_{24} \mathrm{ClO}_{3} \mathrm{~S}$ 487.1129, found 487.1153.<smiles>CC(=O)c1ccc(C(=C([12F])CC(=O)c2ccccc2)c2ccccc2)cc1</smiles>

White solid (82.9 mg, 81\%); mp 162-164 ${ }^{\circ} \mathrm{C} ;{ }^{1} \mathrm{H}$ NMR (400 MHz, $\left.\mathrm{CDCl}_{3}\right) \delta 7.99(\mathrm{~d}, J$ $=7.4 \mathrm{~Hz}, 2 \mathrm{H}), 7.73(\mathrm{~d}, J=8.3 \mathrm{~Hz}, 2 \mathrm{H}), 7.56(\mathrm{t}, J=7.3 \mathrm{~Hz}, 1 \mathrm{H}), 7.49-7.41(\mathrm{~m}, 4 \mathrm{H})$, $7.20(\mathrm{~s}, 5 \mathrm{H}), 7.06(\mathrm{~d}, J=8.1 \mathrm{~Hz}, 2 \mathrm{H}), 6.98(\mathrm{~d}, J=8.1 \mathrm{~Hz}, 2 \mathrm{H}), 4.33(\mathrm{~s}, 2 \mathrm{H}), 3.91$ (s, 3H), 2.31 (s, 3H); $\left.{ }^{13} \mathrm{C} \mathrm{NMR} \mathrm{(100} \mathrm{MHz,} \mathrm{CDCl}_{3}\right) \delta 196.9,166.5,153.1,143.4,142.9$, 139.7, 139.1, 137.2, 136.3, 133.3, 129.4, 129.2, 128.7, 128.62, 128.59, 128.57, 128.2, 128.1, 127.3, 52.1, 40.7, 21.4; HRMS (CI-TOF) m/z: $[\mathrm{M}+\mathrm{H}]^{+}$calcd for $\mathrm{C}_{31} \mathrm{H}_{27} \mathrm{O}_{5} \mathrm{~S}$ 511.1574 , found 511.1590 .<smiles>CC(CC(=O)c1ccccc1)=C(c1ccccc1)c1ccc(-c2ccccc2)cc1</smiles>

White solid (84.4 mg, 80\%); mp 144-146 ${ }^{\circ} \mathrm{C} ;{ }^{1} \mathrm{H}$ NMR (400 MHz, $\left.\mathrm{CDCl}_{3}\right) \delta 8.02$ (d, $J$ $=7.5 \mathrm{~Hz}, 2 \mathrm{H}), 7.58(\mathrm{t}, J=7.3 \mathrm{~Hz}, 1 \mathrm{H}), 7.54-7.43(\mathrm{~m}, 8 \mathrm{H}), 7.37(\mathrm{t}, J=7.0 \mathrm{~Hz}, 1 \mathrm{H})$, 
$7.29-7.21(\mathrm{~m}, 7 \mathrm{H}), 7.03(\mathrm{~d}, J=7.7 \mathrm{~Hz}, 2 \mathrm{H}), 6.97(\mathrm{~d}, J=8.0 \mathrm{~Hz}, 2 \mathrm{H}), 4.35(\mathrm{~s}, 2 \mathrm{H})$, $2.31(\mathrm{~s}, 3 \mathrm{H}) ;{ }^{13} \mathrm{C} \mathrm{NMR}\left(100 \mathrm{MHz}, \mathrm{CDCl}_{3}\right) \delta 197.3,154.1,142.9,140.7,140.6,140.4$, 138.7, 137.5, 137.3, 136.6, 133.3, 129.9, 128.8, 128.61, 128.57, 128.5, 128.3, 128.1, 127.52, 127.45, 126.9, 126.1, 40.8, 21.5; HRMS (CI-TOF) m/z: $[\mathrm{M}+\mathrm{H}]^{+}$calcd for $\mathrm{C}_{35} \mathrm{H}_{29} \mathrm{O}_{3} \mathrm{~S}$ 529.1832, found 529.1827.<smiles>[3H]C(CC(=O)c1ccccc1)=C(c1ccccc1)c1ccc(OC)cc1</smiles>

White solid (61.0 mg, 63\%); mp 175-177 ${ }^{\circ} \mathrm{C} ;{ }^{1} \mathrm{H} \mathrm{NMR}\left(400 \mathrm{MHz}, \mathrm{CDCl}_{3}\right) \delta 8.00$ (d, $J$ $=7.3 \mathrm{~Hz}, 2 \mathrm{H}), 7.56(\mathrm{t}, J=7.3 \mathrm{~Hz}, 1 \mathrm{H}), 7.51-7.42(\mathrm{~m}, 4 \mathrm{H}), 7.20(\mathrm{~s}, 5 \mathrm{H}), 7.02(\mathrm{~d}, J=$ $8.1 \mathrm{~Hz}, 2 \mathrm{H}), 6.89(\mathrm{~d}, J=8.5 \mathrm{~Hz}, 2 \mathrm{H}), 6.59(\mathrm{~d}, J=8.7 \mathrm{~Hz}, 2 \mathrm{H}), 4.31(\mathrm{~s}, 2 \mathrm{H}), 3.77$ (s, 3H), 2.32 (s, 3H); ${ }^{13} \mathrm{C}$ NMR (100 MHz, $\left.\mathrm{CDCl}_{3}\right) \delta 197.5,159.4,154.5,142.8,140.8$, 138.0, 137.7, 136.6, 133.2, 131.0, 130.8, 128.6, 128.5, 128.4, 128.3, 128.0, 127.5, 112.9, 55.2, 40.9, 21.5; HRMS (CI-TOF) m/z: $[\mathrm{M}+\mathrm{H}]^{+}$calcd for $\mathrm{C}_{30} \mathrm{H}_{27} \mathrm{O}_{4} \mathrm{~S} 483.1625$, found 483.1642 .<smiles>O=C(CC([135I])=C(c1ccccc1)c1cccs1)c1ccccc1</smiles>

White solid (0.20 mmol scale, $85.4 \mathrm{mg}$, 93\%; $4.0 \mathrm{mmol}$ scale: $1.67 \mathrm{~g}, 91 \%)$; mp 64-66 ${ }^{\circ} \mathrm{C} ;{ }^{1} \mathrm{H} \mathrm{NMR}\left(400 \mathrm{MHz}, \mathrm{CDCl}_{3}\right) \delta 7.99(\mathrm{~d}, J=7.3 \mathrm{~Hz}, 2 \mathrm{H}), 7.63(\mathrm{~d}, J=8.2 \mathrm{~Hz}, 2 \mathrm{H})$, $7.56(\mathrm{t}, J=7.3 \mathrm{~Hz}, 1 \mathrm{H}), 7.51-7.43(\mathrm{~m}, 3 \mathrm{H}), 7.24(\mathrm{~s}, 5 \mathrm{H}), 7.19(\mathrm{dd}, J=5.0,0.9 \mathrm{~Hz}$, $1 \mathrm{H}), 7.08(\mathrm{~d}, J=8.2 \mathrm{~Hz}, 2 \mathrm{H}), 6.90(\mathrm{dd}, J=5.0,3.7 \mathrm{~Hz}, 1 \mathrm{H}), 4.33(\mathrm{~s}, 2 \mathrm{H}), 2.32(\mathrm{~s}, 3 \mathrm{H})$; ${ }^{13} \mathrm{C} \mathrm{NMR}\left(100 \mathrm{MHz}, \mathrm{CDCl}_{3}\right) \delta 197.2,147.4,143.1,140.5,139.7,139.5,136.9,136.5$, 133.3, 132.1, 129.7, 129.0, 128.7, 128.6, 128.5, 128.3, 128.2, 127.4, 126.0, 41.2, 21.5; HRMS (CI-TOF) m/z: [M+H] ${ }^{+}$calcd for $\mathrm{C}_{27} \mathrm{H}_{23} \mathrm{O}_{3} \mathrm{~S}_{2} 459.1083$, found 459.1096. 
<smiles>COc1ccc(C(=O)CC([125I])=C(c2ccccc2)c2ccc(OC)cc2)cc1</smiles>

Light yellow solid (91.3 mg, 89\%); mp 62-64 ${ }^{\circ} \mathrm{C} ;{ }^{1} \mathrm{H}$ NMR (400 MHz, $\left.\mathrm{CDCl}_{3}\right) \delta 8.02$ $(\mathrm{d}, J=8.7 \mathrm{~Hz}, 2 \mathrm{H}), 7.42(\mathrm{~d}, J=8.1 \mathrm{~Hz}, 2 \mathrm{H}), 7.13(\mathrm{~d}, J=8.5 \mathrm{~Hz}, 3 \mathrm{H}), 7.04(\mathrm{t}, J=7.5$ Hz, 2H), $6.94(\mathrm{dd}, J=17.0,8.0 \mathrm{~Hz}, 6 \mathrm{H}), 6.71(\mathrm{~d}, J=8.6 \mathrm{~Hz}, 2 \mathrm{H}), 4.35$ (s, 2H), 3.84 (s, 3H), $3.68(\mathrm{~s}, 3 \mathrm{H}), 2.29$ (s, 3H); ${ }^{13} \mathrm{C}$ NMR (100 MHz, $\left.\mathrm{CDCl}_{3}\right) \delta$ 195.9, 163.6, 159.7, $154.1,142.7,138.7,137.6,137.5,132.9,130.6,129.6,129.4,129.1,128.5,127.9$, 127.6, 127.3, 113.7, 113.6, 55.4, 55.1, 40.6, 21.4; HRMS (CI-TOF) m/z: $[\mathrm{M}+\mathrm{H}]^{+}$ calcd for $\mathrm{C}_{31} \mathrm{H}_{29} \mathrm{O}_{5} \mathrm{~S} 513.1730$, found 513.1733.<smiles>Cc1ccc(C(=O)C/C([13I])=C(/c2ccccc2)c2ccc(C)cc2)cc1</smiles>

White solid (76.1 mg, 79\%); $\mathrm{mp} 68-70{ }^{\circ} \mathrm{C} ;{ }^{1} \mathrm{H} \mathrm{NMR}\left(400 \mathrm{MHz}, \mathrm{CDCl}_{3}\right) \delta 7.91(\mathrm{~d}, J=$ $8.1 \mathrm{~Hz}, 2 \mathrm{H}), 7.42(\mathrm{~d}, J=8.2 \mathrm{~Hz}, 2 \mathrm{H}), 7.26(\mathrm{~d}, J=7.9 \mathrm{~Hz}, 2 \mathrm{H}), 7.15-6.92(\mathrm{~m}, 11 \mathrm{H})$, $4.32(\mathrm{~s}, 2 \mathrm{H}), 2.41(\mathrm{~s}, 3 \mathrm{H}), 2.30(\mathrm{~s}, 3 \mathrm{H}), 2.22(\mathrm{~s}, 3 \mathrm{H}) ;{ }^{13} \mathrm{C} \mathrm{NMR}\left(100 \mathrm{MHz}, \mathrm{CDCl}_{3}\right) \delta$ 197.0, 154.5, 144.1, 142.8, 138.6, 138.5, 138.0, 137.8, 137.6, 134.1, 129.5, 129.3, 129.1, 128.6, 128.4, 128.0, 127.6, 127.4, 40.8, 21.6, 21.5, 21.1; HRMS (CI-TOF) m/z: $[\mathrm{M}+\mathrm{H}]^{+}$calcd for $\mathrm{C}_{31} \mathrm{H}_{29} \mathrm{O}_{3} \mathrm{~S} 481.1832$, found 481.1844 .<smiles>[13CH3]C(CC(=O)c1ccc(F)cc1)=C(c1ccccc1)c1ccc(F)cc1</smiles> 
White solid (91.8 mg, 94\%); mp 53-55 ${ }^{\circ} \mathrm{C} ;{ }^{1} \mathrm{H}$ NMR (400 MHz, $\left.\mathrm{CDCl}_{3}\right) \delta 8.06(\mathrm{dd}, J$ $=8.3,5.5 \mathrm{~Hz}, 2 \mathrm{H}), 7.38(\mathrm{~d}, J=8.1 \mathrm{~Hz}, 2 \mathrm{H}), 7.23-7.12(\mathrm{~m}, 5 \mathrm{H}), 7.06(\mathrm{t}, J=7.5 \mathrm{~Hz}$, $2 \mathrm{H}), 6.98(\mathrm{~d}, J=8.1 \mathrm{~Hz}, 2 \mathrm{H}), 6.95-6.86(\mathrm{~m}, 4 \mathrm{H}), 4.29(\mathrm{~s}, 2 \mathrm{H}), 2.30(\mathrm{~s}, 3 \mathrm{H}) ;{ }^{13} \mathrm{C}$ NMR (100 MHz, $\left.\mathrm{CDCl}_{3}\right) \delta 195.8,165.9(\mathrm{~d}, J=253.8 \mathrm{~Hz}), 162.7(\mathrm{~d}, J=247.7 \mathrm{~Hz})$, 153.6, 143.0, 138.4, 138.1, 137.2, 136.4 (d, $J=3.4 \mathrm{~Hz}), 132.8$ (d, $J=3 \mathrm{~Hz}), 131.0$ (d, $J=9.3 \mathrm{~Hz}), 129.52,129.45,128.7,128.5,127.92,127.86,127.5,127.4,115.9,115.6$, 115.4, 40.6, 21.4; HRMS (CI-TOF) m/z: $[\mathrm{M}+\mathrm{H}]^{+}$calcd for $\mathrm{C}_{29} \mathrm{H}_{23} \mathrm{~F}_{2} \mathrm{O}_{3} \mathrm{~S}$ 489.1330, found 489.1314 .

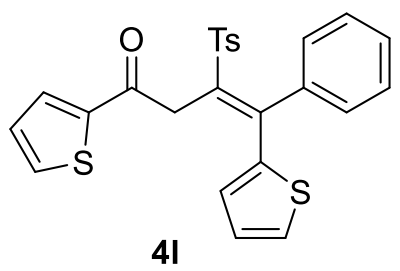

Yellow solid (43.5 mg, 47\%); mp 157-159 ${ }^{\circ} \mathrm{C} ;{ }^{1} \mathrm{H}$ NMR (400 MHz, $\left.\mathrm{CDCl}_{3}\right) \delta 7.87$ (d, $J=3.5 \mathrm{~Hz}, 1 \mathrm{H}), 7.71(\mathrm{~d}, J=4.8 \mathrm{~Hz}, 1 \mathrm{H}), 7.35(\mathrm{~d}, J=8.2 \mathrm{~Hz}, 2 \mathrm{H}), 7.31(\mathrm{~d}, J=5.0 \mathrm{~Hz}$, 1H), $7.22-7.17(\mathrm{~m}, 2 \mathrm{H}), 7.06(\mathrm{t}, J=7.7 \mathrm{~Hz}, 2 \mathrm{H}), 7.03(\mathrm{~d}, J=3.5 \mathrm{~Hz}, 1 \mathrm{H}), 6.95(\mathrm{t}, J$ $=8.6 \mathrm{~Hz}, 4 \mathrm{H}), 6.91-6.87(\mathrm{~m}, 1 \mathrm{H}), 4.63(\mathrm{~s}, 2 \mathrm{H}), 2.31(\mathrm{~s}, 3 \mathrm{H}) ;{ }^{13} \mathrm{C} \mathrm{NMR}(100 \mathrm{MHz}$, $\left.\mathrm{CDCl}_{3}\right) \delta 189.5,147.6,143.1,143.0,142.7,138.3,137.5,136.8,134.1,132.6,130.1$, 129.3, 129.1, 128.7, 128.31, 128.25, 128.0, 127.4, 127.2, 41.8, 21.5; HRMS (CI-TOF) $\mathrm{m} / \mathrm{z}:[\mathrm{M}+\mathrm{H}]^{+}$calcd for $\mathrm{C}_{25} \mathrm{H}_{21} \mathrm{O}_{3} \mathrm{~S}_{3} 465.0647$, found 465.0655 .<smiles>[3H]C(CC(=O)c1ccc(F)cc1)=C(c1ccccc1)c1ccc(OC)cc1</smiles>

White solid (62.1 mg, 62\%); mp 57-59 ${ }^{\circ} \mathrm{C} ;{ }^{1} \mathrm{H}$ NMR (400 MHz, $\left.\mathrm{CDCl}_{3}\right) \delta 8.11-8.03$ (m, 2H), $7.38(\mathrm{~d}, J=8.3 \mathrm{~Hz}, 2 \mathrm{H}), 7.18-7.10(\mathrm{~m}, 5 \mathrm{H}), 7.04(\mathrm{t}, J=7.6 \mathrm{~Hz}, 2 \mathrm{H}), 6.97$ (d, $J=8.1 \mathrm{~Hz}, 2 \mathrm{H}), 6.91(\mathrm{~d}, J=7.4 \mathrm{~Hz}, 2 \mathrm{H}), 6.72(\mathrm{~d}, J=8.8 \mathrm{~Hz}, 2 \mathrm{H}), 4.34(\mathrm{~s}, 2 \mathrm{H})$, $3.71(\mathrm{~s}, 3 \mathrm{H}), 2.31(\mathrm{~s}, 3 \mathrm{H}) ;{ }^{13} \mathrm{C}$ NMR $\left(100 \mathrm{MHz}, \mathrm{CDCl}_{3}\right) \delta 196.2,165.8(\mathrm{~d}, J=253.5$ 
Hz), 159.9, 154.5, 142.8, 138.6, 137.5, 137.3, 133.0 (d, $J=2.9 \mathrm{~Hz}), 132.8,131.0$ (d, $J$ $=9.3 \mathrm{~Hz}), 129.7,129.2,128.6,127.9,127.7,127.4,115.7(\mathrm{~d}, J=21.8 \mathrm{~Hz}), 113.8$, 55.2, 40.9, 21.5; HRMS (CI-TOF) m/z: $[\mathrm{M}+\mathrm{H}]^{+}$calcd for $\mathrm{C}_{30} \mathrm{H}_{26} \mathrm{FO}_{4} \mathrm{~S}$ 501.1530, found 501.1538 .<smiles>COc1ccc(C(=O)CC([123I])=C(c2ccccc2)c2ccc(F)cc2)cc1</smiles>

White solid (19.0 mg, 19\%); mp 58-59 ${ }^{\circ} \mathrm{C} ;{ }^{1} \mathrm{H}$ NMR (400 MHz, $\left.\mathrm{CDCl}_{3}\right) \delta 8.00(\mathrm{~d}, J=$ $8.9 \mathrm{~Hz}, 2 \mathrm{H}), 7.41(\mathrm{~d}, J=8.3 \mathrm{~Hz}, 2 \mathrm{H}), 7.23-7.18(\mathrm{~m}, 2 \mathrm{H}), 7.15(\mathrm{t}, J=7.4 \mathrm{~Hz}, 1 \mathrm{H})$, $7.05(\mathrm{t}, J=7.6 \mathrm{~Hz}, 2 \mathrm{H}), 7.00-6.86(\mathrm{~m}, 8 \mathrm{H}), 4.28(\mathrm{~s}, 2 \mathrm{H}), 3.87(\mathrm{~s}, 3 \mathrm{H}), 2.31(\mathrm{~s}, 3 \mathrm{H})$; ${ }^{13} \mathrm{C} \mathrm{NMR}\left(100 \mathrm{MHz}, \mathrm{CDCl}_{3}\right) \delta 195.7,163.7,162.6(\mathrm{~d}, J=247.5 \mathrm{~Hz}), 153.3,142.9$, 138.8, 138.3, 137.4, $136.6(\mathrm{~d}, J=3.3 \mathrm{~Hz}), 130.6,129.51,129.48(\mathrm{~d}, J=21.2 \mathrm{~Hz})$, 128.6, 128.0, 127.8, 127.5, 115.5 (d, $J=21.5 \mathrm{~Hz}), 113.8,55.5,40.5,21.5$; HRMS (CI-TOF) m/z: $[\mathrm{M}+\mathrm{H}]^{+}$calcd for $\mathrm{C}_{30} \mathrm{H}_{26} \mathrm{FO}_{4} \mathrm{~S}$ 501.1530, found 501.1535.<smiles>[3H]C(CC(=O)c1ccc(C(F)(F)F)cc1)=C(c1ccccc1)c1ccc(OC)cc1</smiles>

White solid (42.0 mg, 38\%); mp 82-84 ${ }^{\circ} \mathrm{C} ;{ }^{1} \mathrm{H}$ NMR (400 MHz, $\left.\mathrm{CDCl}_{3}\right) \delta 8.14$ (d, $J=$ $8.1 \mathrm{~Hz}, 2 \mathrm{H}), 7.75(\mathrm{~d}, J=8.2 \mathrm{~Hz}, 2 \mathrm{H}), 7.37(\mathrm{~d}, J=8.2 \mathrm{~Hz}, 2 \mathrm{H}), 7.19-7.10(\mathrm{~m}, 3 \mathrm{H})$, $7.05(\mathrm{t}, J=7.6 \mathrm{~Hz}, 2 \mathrm{H}), 6.98(\mathrm{~d}, J=8.1 \mathrm{~Hz}, 2 \mathrm{H}), 6.93(\mathrm{~d}, J=7.4 \mathrm{~Hz}, 2 \mathrm{H}), 6.73(\mathrm{~d}, J=$ $8.8 \mathrm{~Hz}, 2 \mathrm{H}), 4.38$ (s, 2H), $3.70(\mathrm{~s}, 3 \mathrm{H}), 2.30$ (s, 3H); ${ }^{13} \mathrm{C} \mathrm{NMR}\left(100 \mathrm{MHz}, \mathrm{CDCl}_{3}\right) \delta$ 197.0, 159.9, 154.8, 142.9, 139.4, 138.4, 137.3, 137.0, 134.4 (q, $J=32.8$ Hz), 132.7, 129.6, 129.1, 128.6, 127.9, 127.8, 127.4, 125.6 (q, $J=3.7 \mathrm{~Hz}), 123.5$ (q, $J=272.8$ $\mathrm{Hz}$ ), 113.8, 55.1, 41.2, 21.4; HRMS (CI-TOF) m/z: $[\mathrm{M}+\mathrm{H}]^{+}$calcd for $\mathrm{C}_{31} \mathrm{H}_{26} \mathrm{~F}_{3} \mathrm{O}_{4} \mathrm{~S}$ 


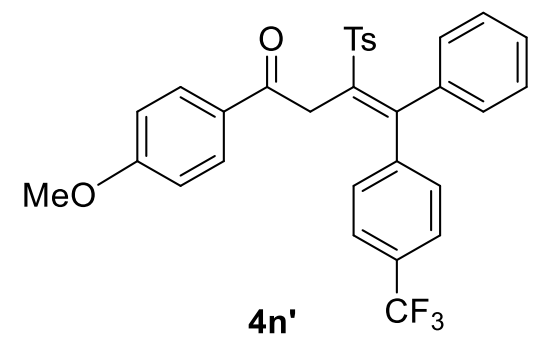

White solid (36.6 mg, 33\%); mp 78-80 ${ }^{\circ} \mathrm{C} ;{ }^{1} \mathrm{H}$ NMR (400 MHz, $\left.\mathrm{CDCl}_{3}\right) \delta 8.02-7.96$ (m, 2H), 7.47 (d, $J=8.1 \mathrm{~Hz}, 2 \mathrm{H}), 7.42(\mathrm{~d}, J=8.3 \mathrm{~Hz}, 2 \mathrm{H}), 7.37(\mathrm{~d}, J=8.1 \mathrm{~Hz}, 2 \mathrm{H})$, $7.16(\mathrm{t}, J=7.4 \mathrm{~Hz}, 1 \mathrm{H}), 7.06(\mathrm{t}, J=7.6 \mathrm{~Hz}, 2 \mathrm{H}), 7.01-6.91(\mathrm{~m}, 6 \mathrm{H}), 4.25(\mathrm{~s}, 2 \mathrm{H})$, $3.83(\mathrm{~s}, 3 \mathrm{H}), 2.30(\mathrm{~s}, 3 \mathrm{H}) ;{ }^{13} \mathrm{C} \mathrm{NMR}\left(100 \mathrm{MHz}, \mathrm{CDCl}_{3}\right) \delta 195.2,163.8,152.6,144.0$, 143.1, 139.7, 137.5, 137.1, 130.6, 130.3 (q, $J=32.6 \mathrm{~Hz}$ ), 129.3, 129.1, 128.6, 127.97, 127.94, 127.8, 127.6, $125.4(\mathrm{q}, J=3.7 \mathrm{~Hz}), 123.6(\mathrm{q}, J=270.6 \mathrm{~Hz}), 113.8,55.4,40.2$, 21.4; HRMS (CI-TOF) m/z: $[\mathrm{M}+\mathrm{H}]^{+}$calcd for $\mathrm{C}_{31} \mathrm{H}_{26} \mathrm{~F}_{3} \mathrm{O}_{4} \mathrm{~S}$ 551.1498, found 551.1511 .<smiles>CC(CC(=O)c1ccccc1)=C(C)c1ccccc1</smiles>

White solid (33.4 mg, 43\%); mp 116-117 ${ }^{\circ} \mathrm{C} ;{ }^{1} \mathrm{H}$ NMR (400 MHz, $\left.\mathrm{CDCl}_{3}\right) \delta 8.05$ (d, $J$ $=8.3 \mathrm{~Hz}, 2 \mathrm{H}), 7.88-7.82(\mathrm{~m}, 2 \mathrm{H}), 7.52(\mathrm{t}, J=7.4 \mathrm{~Hz}, 1 \mathrm{H}), 7.43-7.33(\mathrm{~m}, 4 \mathrm{H}), 7.30$ $-7.21(\mathrm{~m}, 3 \mathrm{H}), 7.20-7.14(\mathrm{~m}, 2 \mathrm{H}), 4.12(\mathrm{~s}, 2 \mathrm{H}), 2.44(\mathrm{~s}, 3 \mathrm{H}), 2.28(\mathrm{~s}, 3 \mathrm{H}) ;{ }^{13} \mathrm{C}$ NMR $\left(100 \mathrm{MHz}, \mathrm{CDCl}_{3}\right) \delta 196.8,153.3,143.9,142.0,138.9,136.3,134.8,133.1,129.5$, 128.6, 128.5, 128.2, 128.1, 127.9, 126.1, 40.9, 23.1, 21.6; HRMS (CI-TOF) m/z: $[\mathrm{M}+\mathrm{H}]^{+}$calcd for $\mathrm{C}_{24} \mathrm{H}_{23} \mathrm{O}_{3} \mathrm{~S} 391.1362$, found 391.1353 . 


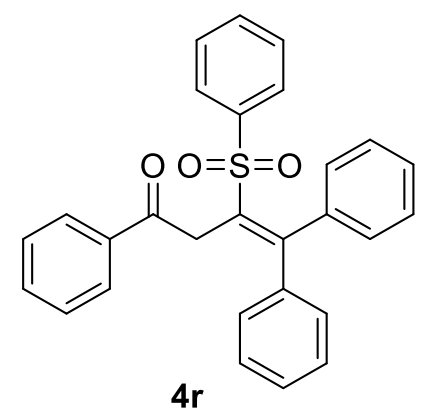

White solid (78.8 mg, 80\%); mp 199-201 ${ }^{\circ} \mathrm{C} ;{ }^{1} \mathrm{H}$ NMR (400 MHz, $\left.\mathrm{CDCl}_{3}\right) \delta 8.02(\mathrm{~d}, J$ $=7.5 \mathrm{~Hz}, 2 \mathrm{H}), 7.58(\mathrm{~d}, J=7.4 \mathrm{~Hz}, 3 \mathrm{H}), 7.48(\mathrm{t}, J=7.5 \mathrm{~Hz}, 2 \mathrm{H}), 7.35(\mathrm{t}, J=7.2 \mathrm{~Hz}$, 1H), $7.13(\mathrm{t}, J=7.2 \mathrm{~Hz}, 7 \mathrm{H}), 7.15-7.11(\mathrm{~m}, 1 \mathrm{H}), 7.04(\mathrm{t}, J=7.4 \mathrm{~Hz}, 2 \mathrm{H}), 6.95(\mathrm{~d}, J$ $=7.3 \mathrm{~Hz}, 2 \mathrm{H}), 4.36(\mathrm{~s}, 2 \mathrm{H}) ;{ }^{13} \mathrm{C} \mathrm{NMR}\left(100 \mathrm{MHz}, \mathrm{CDCl}_{3}\right) \delta 197.4,154.8,140.5,140.4$, 138.13, 138.07, 136.5, 133.3, 132.1, 129.5, 128.6, 128.5, 128.3, 128.02, 127.97, 127.9, 127.7, 127.6, 127.4, 40.7; HRMS (CI-TOF) m/z: $[\mathrm{M}+\mathrm{H}]^{+}$calcd for $\mathrm{C}_{28} \mathrm{H}_{23} \mathrm{O}_{3} \mathrm{~S}$ 439.1362, found 439.1352 .<smiles>COc1ccc(S(=O)(=O)C(CC(=O)c2ccccc2)=C(c2ccccc2)c2ccccc2)cc1</smiles>

White solid (73.3 mg, 78\%); mp 135-137 ${ }^{\circ} \mathrm{C} ;{ }^{1} \mathrm{H}$ NMR (400 MHz, $\left.\mathrm{CDCl}_{3}\right) \delta 8.01(\mathrm{~d}, J$ $=7.4 \mathrm{~Hz}, 2 \mathrm{H}), 7.57(\mathrm{t}, J=7.3 \mathrm{~Hz}, 1 \mathrm{H}), 7.51-7.43(\mathrm{~m}, 4 \mathrm{H}), 7.20(\mathrm{~s}, 5 \mathrm{H}), 7.15(\mathrm{~d}, J=$ $7.2 \mathrm{~Hz}, 1 \mathrm{H}), 7.09$ (t, $J=7.3 \mathrm{~Hz}, 2 \mathrm{H}), 6.98(\mathrm{~d}, J=7.1 \mathrm{~Hz}, 2 \mathrm{H}), 6.66$ (d, $J=9.0 \mathrm{~Hz}$, 2H), 4.33 (s, 2H), 3.79 (s, 3H); ${ }^{13} \mathrm{C}$ NMR (100 MHz, $\left.\mathrm{CDCl}_{3}\right) \delta$ 197.4, 162.6, 154.3, $140.6,138.5,138.4,136.5,133.3,132.0,130.2,129.4,128.6,128.50,128.47,128.3$, 127.7, 127.6, 127.4, 113.3, 55.5, 41.0; HRMS (CI-TOF) m/z: $[\mathrm{M}+\mathrm{H}]^{+}$calcd for $\mathrm{C}_{29} \mathrm{H}_{25} \mathrm{O}_{4} \mathrm{~S} 468.1468$, found 468.1479 . 
<smiles>O=C(CC(=C(c1ccccc1)c1ccccc1)S(=O)(=O)c1ccc(Cl)cc1)c1ccccc1</smiles>

Yellow solid (81.6 mg, 86\%); mp 141-143 ${ }^{\circ} \mathrm{C} ;{ }^{1} \mathrm{H}$ NMR (400 MHz, $\left.\mathrm{CDCl}_{3}\right) \delta 8.01$ (d, $J=7.4 \mathrm{~Hz}, 2 \mathrm{H}), 7.59(\mathrm{t}, J=7.2 \mathrm{~Hz}, 1 \mathrm{H}), 7.53-7.46(\mathrm{~m}, 4 \mathrm{H}), 7.24-7.15(\mathrm{~m}, 8 \mathrm{H})$, $7.09(\mathrm{t}, J=7.5 \mathrm{~Hz}, 2 \mathrm{H}), 6.95(\mathrm{~d}, J=7.3 \mathrm{~Hz}, 2 \mathrm{H}), 4.34(\mathrm{~s}, 2 \mathrm{H}) ;{ }^{13} \mathrm{C} \mathrm{NMR}(100 \mathrm{MHz}$, $\left.\mathrm{CDCl}_{3}\right) \delta 197.5,155.1,140.2,139.0,138.8,138.1,138.0,136.4,133.5,129.53$, $129.46,128.8,128.7,128.6,128.31,128.26,128.0,127.7,127.3,40.8$; HRMS (CI-TOF) m/z: $[\mathrm{M}+\mathrm{H}]^{+}$calcd for $\mathrm{C}_{28} \mathrm{H}_{22} \mathrm{ClO}_{3} \mathrm{~S} 473.0973$, found 473.0982 .<smiles>Cc1cccc(S(=O)(=O)C(=C(CC(=O)c2ccccc2)c2ccccc2)c2ccccc2)c1</smiles>

White solid (74.0 mg, 82\%); mp 132-134 ${ }^{\circ} \mathrm{C} ;{ }^{1} \mathrm{H}$ NMR (400 MHz, $\left.\mathrm{CDCl}_{3}\right) \delta 8.02(\mathrm{~d}, J$ $=7.4 \mathrm{~Hz}, 2 \mathrm{H}), 7.58(\mathrm{t}, J=7.9 \mathrm{~Hz}, 2 \mathrm{H}), 7.48(\mathrm{t}, J=7.6 \mathrm{~Hz}, 2 \mathrm{H}), 7.21(\mathrm{~s}, 5 \mathrm{H}), 7.18(\mathrm{~d}$, $J=7.7 \mathrm{~Hz}, 1 \mathrm{H}), 7.16-7.10(\mathrm{~m}, 3 \mathrm{H}), 7.05(\mathrm{t}, J=7.5 \mathrm{~Hz}, 2 \mathrm{H}), 6.95(\mathrm{~d}, J=7.1 \mathrm{~Hz}, 2 \mathrm{H})$, 4.34 (s, 2H), 2.19 (s, 3H); ${ }^{13} \mathrm{C}$ NMR (100 MHz, $\left.\mathrm{CDCl}_{3}\right) \delta 197.3,154.6,140.4,140.2$, $138.3,138.6,137.8,136.5,133.3,133.1,129.5,128.61,128.58,128.5,128.3,128.2$, 127.6, 127.5, 127.4, 125.2, 40.7, 21.0; HRMS (CI-TOF) m/z: $[\mathrm{M}+\mathrm{H}]^{+}$calcd for $\mathrm{C}_{29} \mathrm{H}_{25} \mathrm{O}_{3} \mathrm{~S} 453.1519$, found 453.1512 . 


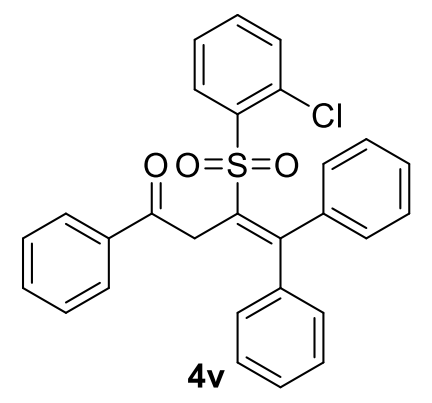

White solid (70.6 mg, 75\%); mp 139-141 ${ }^{\circ} \mathrm{C} ;{ }^{1} \mathrm{H}$ NMR (400 MHz, $\left.\mathrm{CDCl}_{3}\right) \delta 7.92(\mathrm{~d}, J$ $=7.4 \mathrm{~Hz}, 2 \mathrm{H}), 7.52(\mathrm{t}, J=6.9 \mathrm{~Hz}, 1 \mathrm{H}), 7.46-7.34(\mathrm{~m}, 3 \mathrm{H}), 7.31-7.18(\mathrm{~m}, 7 \mathrm{H}), 7.09$ - $6.94(\mathrm{~m}, 5 \mathrm{H}), 6.89(\mathrm{t}, J=7.5 \mathrm{~Hz}, 1 \mathrm{H}), 4.52(\mathrm{~s}, 2 \mathrm{H}) ;{ }^{13} \mathrm{C} \mathrm{NMR}\left(100 \mathrm{MHz}, \mathrm{CDCl}_{3}\right) \delta$ $195.5,154.3,140.4,139.1,137.8,136.8,136.2$, 133.2, 133.1, 131.4, 130.9, 130.7, $128.8,128.6,128.41,128.38,128.3,127.9,127.48,127.45,126.6,40.8$; HRMS (CI-TOF) m/z: $[\mathrm{M}+\mathrm{H}]^{+}$calcd for $\mathrm{C}_{28} \mathrm{H}_{22} \mathrm{ClO}_{3} \mathrm{~S}$ 473.0973, found 473.0962.<smiles>Sc1ccc2ccccc2c1-c1ccccc1</smiles>

$5 a$

White solid (54.2 mg, 76\%); mp 142-144 ${ }^{\circ} \mathrm{C} ;{ }^{1} \mathrm{H}$ NMR (400 MHz, $\left.\mathrm{CDCl}_{3}\right) \delta 8.48$ (d, $J$ $=8.9 \mathrm{~Hz}, 1 \mathrm{H}), 8.03(\mathrm{~d}, J=8.9 \mathrm{~Hz}, 1 \mathrm{H}), 7.90(\mathrm{~d}, J=8.2 \mathrm{~Hz}, 1 \mathrm{H}), 7.55(\mathrm{t}, J=7.4 \mathrm{~Hz}$, 1H), $7.41-7.32(\mathrm{~m}, 2 \mathrm{H}), 7.30-7.24(\mathrm{~m}, 3 \mathrm{H}), 7.18(\mathrm{~d}, J=8.2 \mathrm{~Hz}, 2 \mathrm{H}), 7.01(\mathrm{~d}, J=$ $8.0 \mathrm{~Hz}, 2 \mathrm{H}), 6.93(\mathrm{~d}, J=7.3 \mathrm{~Hz}, 2 \mathrm{H}), 2.32(\mathrm{~s}, 3 \mathrm{H}) ;{ }^{13} \mathrm{C} \mathrm{NMR}\left(100 \mathrm{MHz}, \mathrm{CDCl}_{3}\right) \delta$ 143.2 , 141.1, 138.6, 137.2, 135.1, 134.9, 133.4, 131.0, 128.9, 128.4, 128.1, 127.8, 127.7, 127.63, 127.60, 127.3, 127.0, 123.5, 21.4; HRMS (CI-TOF) m/z: [M+H] $]^{+}$calcd for $\mathrm{C}_{23} \mathrm{H}_{19} \mathrm{O}_{2} \mathrm{~S} 359.1100$, found 359.1110.<smiles>CC(C)(C)c1ccc2c(-c3ccccc3)c(S)ccc2c1</smiles>

$5 b$ 
Yellow solid (64.8 mg, 78\%); mp 181-182 ${ }^{\circ} \mathrm{C} ;{ }^{1} \mathrm{H}$ NMR (400 MHz, $\left.\mathrm{CDCl}_{3}\right) \delta 8.45$ (d, $J=8.9 \mathrm{~Hz}, 1 \mathrm{H}), 8.01(\mathrm{~d}, J=8.9 \mathrm{~Hz}, 1 \mathrm{H}), 7.84(\mathrm{~d}, J=1.8 \mathrm{~Hz}, 1 \mathrm{H}), 7.46(\mathrm{dd}, J=9.1$, $2.0 \mathrm{~Hz}, 1 \mathrm{H}), 7.38$ (t, $J=7.5 \mathrm{~Hz}, 1 \mathrm{H}), 7.29-7.22(\mathrm{~m}, 3 \mathrm{H}), 7.17(\mathrm{~d}, J=8.3 \mathrm{~Hz}, 2 \mathrm{H})$, $7.01(\mathrm{~d}, J=8.1 \mathrm{~Hz}, 2 \mathrm{H}), 6.94-6.91(\mathrm{~m}, 2 \mathrm{H}), 2.34(\mathrm{~s}, 3 \mathrm{H}), 1.38(\mathrm{~s}, 9 \mathrm{H}) ;{ }^{13} \mathrm{C} \mathrm{NMR}$ $\left(100 \mathrm{MHz}, \mathrm{CDCl}_{3}\right) \delta 151.6,143.2,140.8,138.9,136.5,135.3,135.1,131.7,131.0$, $128.9,128.1,127.7,127.4,127.3,126.1,123.7,122.9,34.9,31.0,21.5$; HRMS (CI-TOF) m/z: [M+H] $]^{+}$calcd for $\mathrm{C}_{27} \mathrm{H}_{27} \mathrm{O}_{2} \mathrm{~S}$ 415.1726, found 415.1745.<smiles>Sc1ccc2cc(-c3ccccc3)ccc2c1-c1ccccc1</smiles>

White solid (61.9 mg, 71\%); mp 124-126 ${ }^{\circ} \mathrm{C} ;{ }^{1} \mathrm{H}$ NMR (400 MHz, $\left.\mathrm{CDCl}_{3}\right) \delta 8.52$ (d, $J$ $=8.8 \mathrm{~Hz}, 1 \mathrm{H}), 8.10(\mathrm{~d}, J=10.5 \mathrm{~Hz}, 2 \mathrm{H}), 7.69(\mathrm{~d}, J=7.7 \mathrm{~Hz}, 2 \mathrm{H}), 7.63(\mathrm{~d}, J=8.9 \mathrm{~Hz}$, 1H), $7.49(\mathrm{t}, J=7.4 \mathrm{~Hz}, 2 \mathrm{H}), 7.45-7.36(\mathrm{~m}, 3 \mathrm{H}), 7.31(\mathrm{t}, J=7.5 \mathrm{~Hz}, 2 \mathrm{H}), 7.21(\mathrm{~d}, J$ $=8.0 \mathrm{~Hz}, 2 \mathrm{H}), 7.05(\mathrm{~d}, J=7.9 \mathrm{~Hz}, 2 \mathrm{H}), 6.98(\mathrm{~d}, J=7.5 \mathrm{~Hz}, 2 \mathrm{H}), 2.36(\mathrm{~s}, 3 \mathrm{H}) ;{ }^{13} \mathrm{C}$ NMR (100 MHz, $\left.\mathrm{CDCl}_{3}\right) \delta 143.3,141.10,141.06,139.9,138.7,137.1,135.5,134.9$, 132.6, 131.0, 128.99, 128.97, 128.4, 128.3, 128.0, 127.8, 127.7, 127.4, 126.7, 125.5, 124.1, 21.5; HRMS (CI-TOF) m/z: $[\mathrm{M}+\mathrm{H}]^{+}$calcd for $\mathrm{C}_{29} \mathrm{H}_{23} \mathrm{O}_{2} \mathrm{~S} 435.1413$, found 435.1424 .<smiles>Fc1ccc2ccc(Cl)cc2c1-c1ccc(Br)cc1</smiles>

Light yellow solid (51.2 mg, 65\%); mp 155-157 ${ }^{\circ} \mathrm{C} ;{ }^{1} \mathrm{H}$ NMR (400 MHz, $\left.\mathrm{CDCl}_{3}\right) \delta$ $8.51(\mathrm{~d}, J=8.9 \mathrm{~Hz}, 1 \mathrm{H}), 7.96(\mathrm{~d}, J=8.9 \mathrm{~Hz}, 1 \mathrm{H}), 7.91(\mathrm{~d}, J=2.0 \mathrm{~Hz}, 1 \mathrm{H}), 7.43-$ $7.38(\mathrm{~m}, 1 \mathrm{H}), 7.31-7.25(\mathrm{~m}, 3 \mathrm{H}), 7.22(\mathrm{~d}, J=9.2 \mathrm{~Hz}, 1 \mathrm{H}), 7.17(\mathrm{~d}, J=8.3 \mathrm{~Hz}, 2 \mathrm{H})$, $7.03(\mathrm{~d}, J=8.0 \mathrm{~Hz}, 2 \mathrm{H}), 6.93-6.89(\mathrm{~m}, 2 \mathrm{H}), 2.35(\mathrm{~s}, 3 \mathrm{H}) ;{ }^{13} \mathrm{C} \mathrm{NMR}(100 \mathrm{MHz}$, 
$\left.\mathrm{CDCl}_{3}\right) \delta 143.5,141.2,138.4,137.6,135.8,134.7,134.4,131.8,130.9,129.4,129.0$ 128.0, 127.7, 127.5, 127.2, 126.5, 124.9, 21.5; HRMS (CI-TOF) m/z: $[\mathrm{M}+\mathrm{H}]^{+}$calcd for $\mathrm{C}_{23} \mathrm{H}_{18} \mathrm{ClO}_{2} \mathrm{~S}$ 393.0711, found 393.0712.<smiles>FC(F)(F)c1ccc2c(-c3ccccc3)c(S)ccc2c1</smiles>

$5 e$

White solid (39.1 mg, 46\%); mp 78-80 ${ }^{\circ} \mathrm{C} ;{ }^{1} \mathrm{H}$ NMR (400 MHz, $\left.\mathrm{CDCl}_{3}\right) \delta 8.60$ (d, $J=$ $8.9 \mathrm{~Hz}, 1 \mathrm{H}), 8.23(\mathrm{~s}, 1 \mathrm{H}), 8.16(\mathrm{~d}, J=8.9 \mathrm{~Hz}, 1 \mathrm{H}), 7.51(\mathrm{dd}, J=9.0,1.7 \mathrm{~Hz}, 1 \mathrm{H})$, $7.45-7.40(\mathrm{~m}, 2 \mathrm{H}), 7.29(\mathrm{dd}, J=10.6,4.8 \mathrm{~Hz}, 2 \mathrm{H}), 7.17(\mathrm{~d}, J=8.3 \mathrm{~Hz}, 2 \mathrm{H}), 7.04(\mathrm{~d}$, $J=8.0 \mathrm{~Hz}, 2 \mathrm{H}), 6.94-6.91(\mathrm{~m}, 2 \mathrm{H}), 2.35(\mathrm{~s}, 3 \mathrm{H}) ;{ }^{13} \mathrm{C} \mathrm{NMR}\left(100 \mathrm{MHz}, \mathrm{CDCl}_{3}\right) \delta$ $143.7,141.2,139.5,138.1,134.8,134.2,134.1,130.9,130.1$ (q, $J=32.9 \mathrm{~Hz}), 129.11$, 129.06, 129.0, 128.2, $127.8127 .6,125.5$ (q, $J=4.3 \mathrm{~Hz}), 124.9,123.8$ (q, $J=273.51$ $\mathrm{Hz}), 122.6(\mathrm{q}, J=2.9 \mathrm{~Hz}), 21.5$; HRMS (CI-TOF) $\mathrm{m} / \mathrm{z}:[\mathrm{M}+\mathrm{H}]^{+}$calcd for $\mathrm{C}_{24} \mathrm{H}_{18} \mathrm{~F}_{3} \mathrm{O}_{2} \mathrm{~S} 427.0974$, found 427.0984 .<smiles>Sc1ccc2cc3ccccc3cc2c1-c1ccccc1</smiles>

White solid (57.8 mg, 71\%); mp 212-214 ${ }^{\circ} \mathrm{C} ;{ }^{1} \mathrm{H}$ NMR (400 MHz, $\left.\mathrm{CDCl}_{3}\right) \delta 8.75$ (d, $J$ $=8.6 \mathrm{~Hz}, 1 \mathrm{H}), 8.10(\mathrm{~d}, J=8.6 \mathrm{~Hz}, 1 \mathrm{H}), 7.86-7.75(\mathrm{~m}, 3 \mathrm{H}), 7.40(\mathrm{q}, J=7.7 \mathrm{~Hz}, 2 \mathrm{H})$, $7.23(\mathrm{t}, J=7.6 \mathrm{~Hz}, 2 \mathrm{H}), 7.09(\mathrm{~d}, J=8.1 \mathrm{~Hz}, 2 \mathrm{H}), 7.06(\mathrm{~d}, J=9.0 \mathrm{~Hz}, 1 \mathrm{H}), 7.02-6.95$ (m, 5H), $2.31(\mathrm{~s}, 3 \mathrm{H}) ;{ }^{13} \mathrm{C} \mathrm{NMR}\left(100 \mathrm{MHz}, \mathrm{CDCl}_{3}\right) \delta 142.9,141.1,139.4,138.7$, $137.8,136.5,133.8,131.5,131.0,130.9,130.3,128.9,128.82$, 128.75, 128.2, 128.1, 127.8, 127.2, 126.7, 126.5, 125.9, 125.6, 21.5; HRMS (CI-TOF) m/z: $[\mathrm{M}+\mathrm{H}]^{+}$calcd for $\mathrm{C}_{27} \mathrm{H}_{21} \mathrm{O}_{2} \mathrm{~S}$ 409.1257, found 409.1266. 


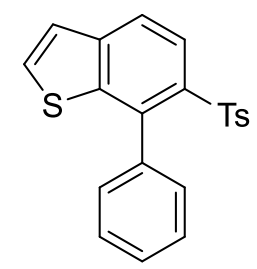

$5 \mathrm{~g}$

Yellow solid (44.6 mg, 61\%); mp 102-104 ${ }^{\circ} \mathrm{C} ;{ }^{1} \mathrm{H}$ NMR (400 MHz, $\left.\mathrm{CDCl}_{3}\right) \delta 8.42(\mathrm{~d}$, $J=8.5 \mathrm{~Hz}, 1 \mathrm{H}), 7.96(\mathrm{~d}, J=8.5 \mathrm{~Hz}, 1 \mathrm{H}), 7.59(\mathrm{~d}, J=5.5 \mathrm{~Hz}, 1 \mathrm{H}), 7.43(\mathrm{~d}, J=5.5 \mathrm{~Hz}$, $1 \mathrm{H}), 7.40(\mathrm{~d}, J=7.5 \mathrm{~Hz}, 1 \mathrm{H}), 7.30(\mathrm{t}, J=7.6 \mathrm{~Hz}, 2 \mathrm{H}), 7.18(\mathrm{~d}, J=8.2 \mathrm{~Hz}, 2 \mathrm{H}), 7.05$ $(\mathrm{d}, J=7.4 \mathrm{~Hz}, 2 \mathrm{H}), 7.01(\mathrm{~d}, J=8.1 \mathrm{~Hz}, 2 \mathrm{H}), 2.33(\mathrm{~s}, 3 \mathrm{H}) ;{ }^{13} \mathrm{C}$ NMR $(100 \mathrm{MHz}$, $\left.\mathrm{CDCl}_{3}\right) \delta 143.9,143.3,142.7,138.7,137.1,136.1,135.1,132.1,129.9,129.0,128.4$, $127.8,127.7,124.5,123.9,122.5,21.5$; HRMS $(\mathrm{CI}-\mathrm{TOF}) \mathrm{m} / \mathrm{z}:[\mathrm{M}+\mathrm{H}]^{+}$calcd for $\mathrm{C}_{21} \mathrm{H}_{17} \mathrm{O}_{2} \mathrm{~S}_{2} 365.0664$, found 365.0659.<smiles>Fc1ccccc1-c1c(F)ccc2ccccc12</smiles>

$5 \mathrm{~h}$

White solid (44.9 mg, 62\%); mp 172-174 ${ }^{\circ} \mathrm{C} ;{ }^{1} \mathrm{H}$ NMR $\left(400 \mathrm{MHz}, \mathrm{CDCl}_{3}\right) \delta 8.47(\mathrm{~d}, J$ $=8.8 \mathrm{~Hz}, 1 \mathrm{H}), 8.08(\mathrm{~d}, J=8.9 \mathrm{~Hz}, 1 \mathrm{H}), 7.94(\mathrm{~d}, J=8.2 \mathrm{~Hz}, 1 \mathrm{H}), 7.59(\mathrm{t}, J=7.4 \mathrm{~Hz}$, 1H), $7.42(\mathrm{dd}, J=16.7,8.7 \mathrm{~Hz}, 2 \mathrm{H}), 7.31-7.25(\mathrm{~m}, 3 \mathrm{H}), 7.19$ (t, $J=7.4 \mathrm{~Hz}, 1 \mathrm{H})$, $7.13(\mathrm{t}, J=7.2 \mathrm{~Hz}, 1 \mathrm{H}), 7.07(\mathrm{~d}, J=7.8 \mathrm{~Hz}, 2 \mathrm{H}), 6.92(\mathrm{t}, J=8.8 \mathrm{~Hz}, 1 \mathrm{H}), 2.35(\mathrm{~s}, 3 \mathrm{H})$; ${ }^{13} \mathrm{C}$ NMR $\left(100 \mathrm{MHz}, \mathrm{CDCl}_{3}\right) \delta 160.0(\mathrm{~d}, J=246.2 \mathrm{~Hz}), 143.6,138.1,137.9,135.1$, $134.8,133.3(\mathrm{~d}, J=2.7 \mathrm{~Hz}), 133.0,130.5(\mathrm{~d}, J=7.9 \mathrm{~Hz}), 129.10,129.05,128.7$, 128.1, 127.6, 127.5, 126.8, 123.8, $123.4(\mathrm{~d}, J=3.6 \mathrm{~Hz}), 122.7(\mathrm{~d}, J=16.7 \mathrm{~Hz}), 115.0$ (d, $J=21.6 \mathrm{~Hz}$ ), 21.5; HRMS (CI-TOF) $\mathrm{m} / \mathrm{z}$ : $[\mathrm{M}+\mathrm{H}]^{+}$calcd for $\mathrm{C}_{23} \mathrm{H}_{18} \mathrm{FO}_{2} \mathrm{~S} 377.1006$, found 377.1006 . 


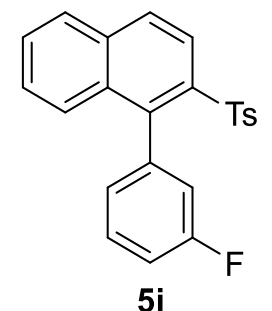

White solid (0.20 mmol scale, $52.9 \mathrm{mg}, 73 \%$; $4.0 \mathrm{mmol} \mathrm{scale,} 1.06 \mathrm{~g}, 70 \%)$; mp 98-100 ${ }^{\circ} \mathrm{C} ;{ }^{1} \mathrm{H}$ NMR (400 MHz, $\left.\mathrm{CDCl}_{3}\right) \delta 8.48(\mathrm{~d}, J=8.9 \mathrm{~Hz}, 1 \mathrm{H}), 8.07(\mathrm{~d}, J=8.9$ $\mathrm{Hz}, 1 \mathrm{H}), 7.93(\mathrm{~d}, J=8.2 \mathrm{~Hz}, 1 \mathrm{H}), 7.61-7.57(\mathrm{~m}, 1 \mathrm{H}), 7.42-7.37(\mathrm{~m}, 1 \mathrm{H}), 7.33-$ $7.26(\mathrm{~m}, 2 \mathrm{H}), 7.23(\mathrm{~d}, J=8.3 \mathrm{~Hz}, 2 \mathrm{H}), 7.11(\mathrm{dd}, J=8.4,2.4 \mathrm{~Hz}, 1 \mathrm{H}), 7.07(\mathrm{~d}, J=8.1$ $\mathrm{Hz}, 2 \mathrm{H}), 6.85(\mathrm{~d}, J=7.6 \mathrm{~Hz}, 1 \mathrm{H}), 6.48(\mathrm{dd}, J=9.2,1.8 \mathrm{~Hz}, 1 \mathrm{H}), 2.36(\mathrm{~s}, 3 \mathrm{H}) ;{ }^{13} \mathrm{C}$ NMR $\left(100 \mathrm{MHz}, \mathrm{CDCl}_{3}\right) \delta 163.1,160.7,143.73,143.72,139.38,139.37,138.54$, $138.48,137.40,137.37,137.1,137.0,136.98,136.96,135.2,133.1,129.1,129.0$, 128.9, 128.6, 128.0, 127.7, 127.3, 127.2, 127.1, 123.5, 118.0, 117.8, 114.9, 114.7, 21.5; HRMS (CI-TOF) m/z: $[\mathrm{M}+\mathrm{H}]^{+}$calcd for $\mathrm{C}_{23} \mathrm{H}_{18} \mathrm{FO}_{2} \mathrm{~S}$ 377.1006, found 377.1009 .

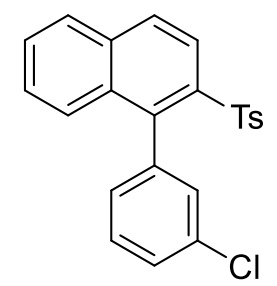

$5 \mathbf{j}$

Yellow solid (46.2 mg, 59\%); mp 136-138 ${ }^{\circ} \mathrm{C} ;{ }^{1} \mathrm{H}$ NMR (400 MHz, $\left.\mathrm{CDCl}_{3}\right) \delta 8.50(\mathrm{~d}$, $J=8.8 \mathrm{~Hz}, 1 \mathrm{H}), 8.07(\mathrm{~d}, J=8.9 \mathrm{~Hz}, 1 \mathrm{H}), 7.92(\mathrm{~d}, J=8.2 \mathrm{~Hz}, 1 \mathrm{H}), 7.58(\mathrm{t}, J=7.5 \mathrm{~Hz}$, 1H), $7.41-7.30(\mathrm{~m}, 3 \mathrm{H}), 7.26(\mathrm{~d}, J=8.7 \mathrm{~Hz}, 1 \mathrm{H}), 7.20(\mathrm{~d}, J=7.9 \mathrm{~Hz}, 2 \mathrm{H}), 7.09$ (t, $J$ $=7.5 \mathrm{~Hz}, 3 \mathrm{H}), 6.55(\mathrm{~s}, 1 \mathrm{H}), 2.37(\mathrm{~s}, 3 \mathrm{H}) ;{ }^{13} \mathrm{C} \mathrm{NMR}\left(100 \mathrm{MHz}, \mathrm{CDCl}_{3}\right) \delta 143.8,139.2$, 138.3, 137.4, 136.6, 135.1, 133.3, 133.0, 130.4, 129.7, 129.2, 128.8, 128.6, 128.0, 127.9, 127.5, 127.3, 127.2, 123.4, 21.5; HRMS (CI-TOF) m/z: $[\mathrm{M}+\mathrm{H}]^{+}$calcd for $\mathrm{C}_{23} \mathrm{H}_{18} \mathrm{ClO}_{2} \mathrm{~S}$ 393.0711, found 393.0706. 
<smiles>Sc1ccc2ccccc2c1-c1ccc(Cl)cc1</smiles>

5k

Yellow solid (47.9 mg, 61\%); mp 140-142 ${ }^{\circ} \mathrm{C} ;{ }^{1} \mathrm{H}$ NMR (400 MHz, $\left.\mathrm{CDCl}_{3}\right) \delta 8.47$ (d, $J=8.9 \mathrm{~Hz}, 1 \mathrm{H}), 8.07(\mathrm{~d}, J=8.8 \mathrm{~Hz}, 1 \mathrm{H}), 7.94(\mathrm{~d}, J=8.2 \mathrm{~Hz}, 1 \mathrm{H}), 7.62-7.57(\mathrm{~m}$, 1H), $7.42-7.37(\mathrm{~m}, 1 \mathrm{H}), 7.30-7.21(\mathrm{~m}, 5 \mathrm{H}), 7.08(\mathrm{~d}, J=8.1 \mathrm{~Hz}, 2 \mathrm{H}), 6.90-6.86$ $(\mathrm{m}, 2 \mathrm{H}), 2.37(\mathrm{~s}, 3 \mathrm{H}) ;{ }^{13} \mathrm{C} \mathrm{NMR}\left(100 \mathrm{MHz}, \mathrm{CDCl}_{3}\right) \delta 143.6,139.7,138.6,137.5$, 135.2, 134.1, 133.4, 133.2, 132.4, 129.1, 128.6, 128.6, 128.0, 127.61, 127.59, 127.3, 127.3, 123.6, 21.5; HRMS (CI-TOF) m/z: $[\mathrm{M}+\mathrm{H}]^{+}$calcd for $\mathrm{C}_{23} \mathrm{H}_{18} \mathrm{ClO}_{2} \mathrm{~S}$ 393.0711, found 393.0709.<smiles>Sc1ccc2ccccc2c1-c1ccc(-c2ccccc2)cc1</smiles>

White solid (54.9 mg, 63\%); mp 161-163 ${ }^{\circ} \mathrm{C} ;{ }^{1} \mathrm{H}$ NMR (400 MHz, $\left.\mathrm{CDCl}_{3}\right) \delta 8.52$ (d, $J$ $=8.9 \mathrm{~Hz}, 1 \mathrm{H}), 8.07(\mathrm{~d}, J=8.9 \mathrm{~Hz}, 1 \mathrm{H}), 7.95(\mathrm{~d}, J=8.2 \mathrm{~Hz}, 1 \mathrm{H}), 7.69(\mathrm{~d}, J=7.5 \mathrm{~Hz}$, 2H), $7.62-7.58(\mathrm{~m}, 1 \mathrm{H}), 7.52(\mathrm{dd}, J=16.1,7.8 \mathrm{~Hz}, 4 \mathrm{H}), 7.42(\mathrm{dd}, J=13.6,5.2 \mathrm{~Hz}$, 3H), $7.22(\mathrm{~d}, J=7.8 \mathrm{~Hz}, 2 \mathrm{H}), 7.01(\mathrm{~d}, J=7.8 \mathrm{~Hz}, 4 \mathrm{H}), 2.35(\mathrm{~s}, 3 \mathrm{H}) ;{ }^{13} \mathrm{C}$ NMR $(100$ $\left.\mathrm{MHz}, \mathrm{CDCl}_{3}\right) \delta 143.3,140.8,140.7,140.5,138.6,137.6,135.3,133.9,133.5,131.5$, $128.94,128.93,128.5,128.2$, 127.9, 127.74, 127.70, 127.6, 127.13, 127.05, 126.0, 123.6, 21.6; HRMS (CI-TOF) m/z: $[\mathrm{M}+\mathrm{H}]^{+}$calcd for $\mathrm{C}_{29} \mathrm{H}_{23} \mathrm{O}_{2} \mathrm{~S}$ 435.1413, found 435.1408 . 


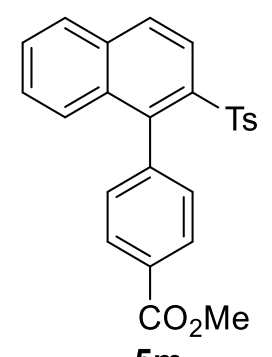

$5 \mathrm{~m}$

White solid (45.8 mg, 55\%); mp 124-126 ${ }^{\circ} \mathrm{C} ;{ }^{1} \mathrm{H}$ NMR (400 MHz, $\left.\mathrm{CDCl}_{3}\right) \delta 8.47$ (d, $J$ $=8.9 \mathrm{~Hz}, 1 \mathrm{H}), 8.08(\mathrm{~d}, J=8.9 \mathrm{~Hz}, 1 \mathrm{H}), 7.97(\mathrm{~d}, J=8.3 \mathrm{~Hz}, 2 \mathrm{H}), 7.94(\mathrm{~d}, J=8.4 \mathrm{~Hz}$, 1H), $7.62-7.57(\mathrm{~m}, 1 \mathrm{H}), 7.40-7.35(\mathrm{~m}, 1 \mathrm{H}), 7.23-7.18(\mathrm{~m}, 3 \mathrm{H}), 7.07-7.03(\mathrm{~m}$, 4H), 4.00 (s, 3H), 2.36 (s, 3H); ${ }^{13} \mathrm{C}$ NMR (100 MHz, $\left.\mathrm{CDCl}_{3}\right) \delta$ 166.9, 143.8, 140.1, 139.9, 138.6, 137.2, 135.1, 132.9, 131.1, 129.6, 129.2, 128.73, 128.65, 128.6, 128.0, 127.7, 127.4, 127.3, 123.7, 52.3, 21.5; HRMS (CI-TOF) m/z: $[\mathrm{M}+\mathrm{H}]^{+}$calcd for $\mathrm{C}_{25} \mathrm{H}_{21} \mathrm{O}_{4} \mathrm{~S}$ 417.1155, found 417.1155.

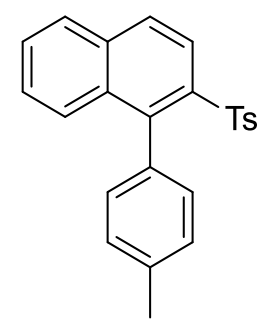

$5 n$

White solid (39.5 mg, 53\%); mp 149-151 ${ }^{\circ} \mathrm{C} ;{ }^{1} \mathrm{H}$ NMR (400 MHz, $\left.\mathrm{CDCl}_{3}\right) \delta 8.46$ (d, $J$ $=8.9 \mathrm{~Hz}, 1 \mathrm{H}), 8.03(\mathrm{~d}, J=8.9 \mathrm{~Hz}, 1 \mathrm{H}), 7.92(\mathrm{~d}, J=8.2 \mathrm{~Hz}, 1 \mathrm{H}), 7.57(\mathrm{dd}, J=7.7,6.6$ $\mathrm{Hz}, 1 \mathrm{H}), 7.35$ (q, $J=8.9 \mathrm{~Hz}, 2 \mathrm{H}), 7.21(\mathrm{~d}, J=8.0 \mathrm{~Hz}, 2 \mathrm{H}), 7.08(\mathrm{~d}, J=7.7 \mathrm{~Hz}, 2 \mathrm{H})$, $7.03(\mathrm{~d}, J=8.0 \mathrm{~Hz}, 2 \mathrm{H}), 6.82(\mathrm{~d}, J=7.6 \mathrm{~Hz}, 2 \mathrm{H}), 2.45(\mathrm{~s}, 3 \mathrm{H}), 2.35(\mathrm{~s}, 3 \mathrm{H}) ;{ }^{13} \mathrm{C} \mathrm{NMR}$ $\left(100 \mathrm{MHz}, \mathrm{CDCl}_{3}\right) \delta 143.3,141.4,138.8,137.5,137.4,135.2,133.7,131.9,130.9$, $128.9,128.4,128.0,128.0,127.8,127.77,127.75,127.0,123.6,21.5,21.4$; HRMS (CI-TOF) m/z: [M+H] $]^{+}$calcd for $\mathrm{C}_{24} \mathrm{H}_{21} \mathrm{O}_{2} \mathrm{~S} 373.1257$, found 373.1259. 


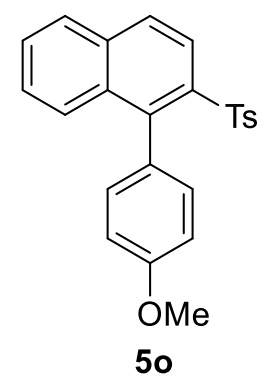

White solid (40.4 mg, 52\%); mp 118-119 ${ }^{\circ} \mathrm{C} ;{ }^{1} \mathrm{H}$ NMR (400 MHz, $\left.\mathrm{CDCl}_{3}\right) \delta 8.48$ (d, $J$ $=8.9 \mathrm{~Hz}, 1 \mathrm{H}), 8.03(\mathrm{~d}, J=8.9 \mathrm{~Hz}, 1 \mathrm{H}), 7.92(\mathrm{~d}, J=8.2 \mathrm{~Hz}, 1 \mathrm{H}), 7.57(\mathrm{ddd}, J=8.1$, 5.7, $2.3 \mathrm{~Hz}, 1 \mathrm{H}), 7.37(\mathrm{dd}, J=5.7,1.0 \mathrm{~Hz}, 2 \mathrm{H}), 7.21(\mathrm{~d}, J=8.3 \mathrm{~Hz}, 2 \mathrm{H}), 7.04(\mathrm{~d}, J=$ $8.0 \mathrm{~Hz}, 2 \mathrm{H}), 6.86-6.79(\mathrm{~m}, 4 \mathrm{H}), 3.90(\mathrm{~s}, 3 \mathrm{H}), 2.35$ (s, 3H); ${ }^{13} \mathrm{C}$ NMR (100 MHz, $\left.\mathrm{CDCl}_{3}\right) \delta 159.2,143.2,141.1,138.7,137.6,135.2,133.9,132.2,128.9,128.4,128.0$ 127.8, 127.7, 127.6, 127.0, 126.9, 123.6, 112.8, 55.3, 21.5; HRMS (CI-TOF) m/z: $[\mathrm{M}+\mathrm{H}]^{+}$calcd for $\mathrm{C}_{24} \mathrm{H}_{21} \mathrm{O}_{3} \mathrm{~S} 389.1206$, found 389.1209.<smiles>Cc1cc(S)c(-c2ccc(F)cc2)c2ccccc12</smiles>

5s

White solid (50.6 mg, 68\%); mp 179-181 ${ }^{\circ} \mathrm{C} ;{ }^{1} \mathrm{H}$ NMR (400 MHz, $\left.\mathrm{CDCl}_{3}\right) \delta 8.34$ (s, $1 \mathrm{H}), 8.06(\mathrm{~d}, J=8.5 \mathrm{~Hz}, 1 \mathrm{H}), 7.60(\mathrm{t}, J=7.4 \mathrm{~Hz}, 1 \mathrm{H}), 7.40-7.31(\mathrm{~m}, 3 \mathrm{H}), 7.25(\mathrm{t}, J$ $=7.6 \mathrm{~Hz}, 2 \mathrm{H}), 7.18(\mathrm{~d}, J=8.1 \mathrm{~Hz}, 2 \mathrm{H}), 7.01(\mathrm{~d}, J=8.0 \mathrm{~Hz}, 2 \mathrm{H}), 6.91(\mathrm{~d}, J=7.5 \mathrm{~Hz}$, 2H), $2.84(\mathrm{~s}, 3 \mathrm{H}), 2.33$ (s, 3H); ${ }^{13} \mathrm{C} \mathrm{NMR}\left(100 \mathrm{MHz}, \mathrm{CDCl}_{3}\right) \delta$ 143.2, 139.3, 138.7, $136.5,135.10,135.05,134.4,133.5,131.2,128.9,128.30,128.27,127.6,127.3,126.6$, 124.0, 123.7, 21.5, 19.7; HRMS (CI-TOF) m/z: $[\mathrm{M}+\mathrm{H}]^{+}$calcd for $\mathrm{C}_{24} \mathrm{H}_{21} \mathrm{O}_{2} \mathrm{~S}$ 373.1257 , found 373.1266 . 
<smiles>CCc1cc(F)c(-c2ccc(Br)cc2)c2ccccc12</smiles>

White solid (44.2 mg, 57\%); mp 127-129 ${ }^{\circ} \mathrm{C} ;{ }^{1} \mathrm{H}$ NMR (400 MHz, $\left.\mathrm{CDCl}_{3}\right) \delta 8.37$ (s, $1 \mathrm{H}), 8.13(\mathrm{~d}, J=8.6 \mathrm{~Hz}, 1 \mathrm{H}), 7.59(\mathrm{ddd}, J=8.4,6.2,1.8 \mathrm{~Hz}, 1 \mathrm{H}), 7.40-7.29(\mathrm{~m}, 3 \mathrm{H})$, $7.25(\mathrm{dd}, J=8.3,7.0 \mathrm{~Hz}, 2 \mathrm{H}), 7.17(\mathrm{~d}, J=8.3 \mathrm{~Hz}, 2 \mathrm{H}), 7.01(\mathrm{~d}, J=8.1 \mathrm{~Hz}, 2 \mathrm{H}), 6.94$ - $6.89(\mathrm{~m}, 2 \mathrm{H}), 3.25(\mathrm{q}, J=7.5 \mathrm{~Hz}, 2 \mathrm{H}), 2.33(\mathrm{~s}, 3 \mathrm{H}), 1.50(\mathrm{t}, J=7.5 \mathrm{~Hz}, 3 \mathrm{H}) ;{ }^{13} \mathrm{C}$ NMR $\left(100 \mathrm{MHz}, \mathrm{CDCl}_{3}\right) \delta 143.1,140.9,139.2,138.8,136.7,135.2,133.8,133.7$, $131.2,128.9,128.5,128.2,127.60,127.58,127.3,126.5,123.6,122.2,26.2,21.5$, 14.8; HRMS (CI-TOF) m/z: $[\mathrm{M}+\mathrm{H}]^{+}$calcd for $\mathrm{C}_{25} \mathrm{H}_{23} \mathrm{O}_{2} \mathrm{~S} 387.1413$, found 387.1415.<smiles>CC(C)c1cc(F)c(-c2ccc(Br)cc2)c2ccccc12</smiles>

White solid (43.9 mg, 55\%); mp 154-156 ${ }^{\circ} \mathrm{C} ;{ }^{1} \mathrm{H}$ NMR (400 MHz, $\left.\mathrm{CDCl}_{3}\right) \delta 8.44$ (s, $1 \mathrm{H}), 8.21(\mathrm{~d}, J=8.7 \mathrm{~Hz}, 1 \mathrm{H}), 7.60(\mathrm{ddd}, J=8.4,5.5,2.5 \mathrm{~Hz}, 1 \mathrm{H}), 7.35$ (ddd, $J=10.1$, 6.0, 4.7 Hz, 3H), $7.24(\mathrm{dd}, J=9.4,5.8 \mathrm{~Hz}, 2 \mathrm{H}), 7.17(\mathrm{~d}, J=8.3 \mathrm{~Hz}, 2 \mathrm{H}), 7.01(\mathrm{~d}, J=$ $8.1 \mathrm{~Hz}, 2 \mathrm{H}), 6.93-6.89(\mathrm{~m}, 2 \mathrm{H}), 3.92-3.80(\mathrm{~m}, 1 \mathrm{H}), 2.34(\mathrm{~s}, 3 \mathrm{H}), 1.54(\mathrm{~d}, J=6.8$ $\mathrm{Hz}, 6 \mathrm{H}) .{ }^{13} \mathrm{C}$ NMR $\left(100 \mathrm{MHz}, \mathrm{CDCl}_{3}\right) \delta 145.3,143.1,138.9,138.8,136.9,135.2$, 133.9, 133.3, 131.2, 128.9, 128.6, 128.2, 127.59, 127.56, 127.3, 126.3, 123.2, 119.4, 29.0, 23.4, 21.5; HRMS (CI-TOF) m/z: $[\mathrm{M}+\mathrm{H}]^{+}$calcd for $\mathrm{C}_{26} \mathrm{H}_{25} \mathrm{O}_{2} \mathrm{~S} 401.1570$, found 401.1578 . 
<smiles>Sc1cc(C2CCCCC2)c2ccccc2c1-c1ccccc1</smiles>

5v

White solid (45.9 mg, 52\%); mp 181-183 ${ }^{\circ} \mathrm{C} ;{ }^{1} \mathrm{H}$ NMR (400 MHz, $\left.\mathrm{CDCl}_{3}\right) \delta 8.42$ (s, $1 \mathrm{H}), 8.20(\mathrm{~d}, J=8.6 \mathrm{~Hz}, 1 \mathrm{H}), 7.60(\mathrm{ddd}, J=8.4,5.9,2.1 \mathrm{~Hz}, 1 \mathrm{H}), 7.40-7.29(\mathrm{~m}, 3 \mathrm{H})$, $7.27-7.21(\mathrm{~m}, 2 \mathrm{H}), 7.16(\mathrm{~d}, J=8.3 \mathrm{~Hz}, 2 \mathrm{H}), 7.01(\mathrm{~d}, J=8.0 \mathrm{~Hz}, 2 \mathrm{H}), 6.91(\mathrm{~d}, J=$ $7.1 \mathrm{~Hz}, 2 \mathrm{H}), 3.49-3.37(\mathrm{~m}, 1 \mathrm{H}), 2.34(\mathrm{~s}, 3 \mathrm{H}), 2.18-2.09(\mathrm{~m}, 2 \mathrm{H}), 2.05-1.96(\mathrm{~m}$, 2H), $1.94-1.87(\mathrm{~m}, 1 \mathrm{H}), 1.83-1.70(\mathrm{~m}, 2 \mathrm{H}), 1.68-1.53(\mathrm{~m}, 2 \mathrm{H}), 1.50-1.39(\mathrm{~m}$, 1H). ${ }^{13} \mathrm{C}$ NMR $\left(100 \mathrm{MHz}, \mathrm{CDCl}_{3}\right) \delta 144.6,143.1,138.84,138.83,136.8,135.3,134.0$, 133.3, 131.3, 128.9, 128.6, 128.1, 127.61, 127.56, 127.3, 126.3, 123.2, 119.9, 39.6, 34.0, 27.2, 26.3, 21.5; HRMS (CI-TOF) m/z: $[\mathrm{M}+\mathrm{H}]^{+}$calcd for $\mathrm{C}_{29} \mathrm{H}_{29} \mathrm{O}_{2} \mathrm{~S}$ 441.1883, found 441.1886 .<smiles>O=S(=O)(c1ccccc1)c1ccc2ccccc2c1-c1ccccc1</smiles>

$5 w$

Yellow solid (43.5 mg, 63\%); mp 135-137 ${ }^{\circ} \mathrm{C} ;{ }^{1} \mathrm{H}$ NMR (400 MHz, $\left.\mathrm{CDCl}_{3}\right) \delta 8.51(\mathrm{~d}$, $J=8.8 \mathrm{~Hz}, 1 \mathrm{H}), 8.06(\mathrm{~d}, J=8.9 \mathrm{~Hz}, 1 \mathrm{H}), 7.93(\mathrm{~d}, J=8.2 \mathrm{~Hz}, 1 \mathrm{H}), 7.58(\mathrm{t}, J=7.4 \mathrm{~Hz}$, 1H), $7.43-7.34(\mathrm{~m}, 3 \mathrm{H}), 7.31-7.19(\mathrm{~m}, 7 \mathrm{H}), 6.91(\mathrm{~d}, J=7.5 \mathrm{~Hz}, 2 \mathrm{H}) ;{ }^{13} \mathrm{C} \mathrm{NMR}$ $\left(100 \mathrm{MHz}, \mathrm{CDCl}_{3}\right) \delta 141.5,141.3,136.9,135.2,134.7,133.4,132.4,131.0,128.5$, 128.4, 128.2, 127.9, 127.7, 127.62, 127.55, 127.4, 127.1, 123.5; HRMS (CI-TOF) m/z: $[\mathrm{M}+\mathrm{H}]^{+}$calcd for $\mathrm{C}_{22} \mathrm{H}_{17} \mathrm{O}_{2} \mathrm{~S} 345.0944$, found 345.0939. 


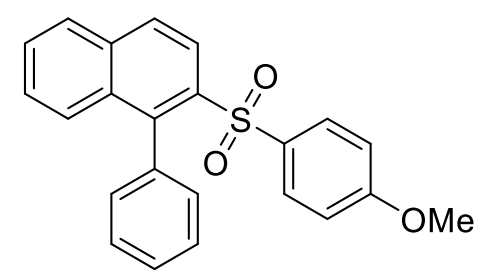

$5 x$

White solid (49.5 mg, 66\%); mp 129-131 ${ }^{\circ} \mathrm{C} ;{ }^{1} \mathrm{H}$ NMR (400 MHz, $\left.\mathrm{CDCl}_{3}\right) \delta 8.48(\mathrm{~d}, J$ $=8.8 \mathrm{~Hz}, 1 \mathrm{H}), 8.04(\mathrm{~d}, J=8.9 \mathrm{~Hz}, 1 \mathrm{H}), 7.92(\mathrm{~d}, J=8.2 \mathrm{~Hz}, 1 \mathrm{H}), 7.60-7.54(\mathrm{~m}, 1 \mathrm{H})$, $7.43-7.36(\mathrm{~m}, 2 \mathrm{H}), 7.35-7.27(\mathrm{~m}, 3 \mathrm{H}), 7.24-7.18(\mathrm{~m}, 2 \mathrm{H}), 6.99-6.93(\mathrm{~m}, 2 \mathrm{H})$, $6.73-6.67(\mathrm{~m}, 2 \mathrm{H}), 3.80(\mathrm{~s}, 3 \mathrm{H}) ;{ }^{13} \mathrm{C} \mathrm{NMR}\left(100 \mathrm{MHz}, \mathrm{CDCl}_{3}\right) \delta 162.8,141.0,137.5$, $135.2,135.0,133.5,133.2,131.1,129.9,128.4,128.2,127.9,127.8,127.7,127.4$, 127.0, 123.6, 113.6, 55.6; HRMS (CI-TOF) m/z: $[\mathrm{M}+\mathrm{H}]^{+}$calcd for $\mathrm{C}_{23} \mathrm{H}_{19} \mathrm{O}_{3} \mathrm{~S}$ 375.1049, found 375.1042.<smiles>O=S(=O)(c1ccc(Cl)cc1)c1ccc2ccccc2c1-c1ccccc1</smiles>

$5 y$

Light yellow solid (51.6 mg, 68\%); mp 142-143 ${ }^{\circ} \mathrm{C} ;{ }^{1} \mathrm{H} \mathrm{NMR}\left(400 \mathrm{MHz}, \mathrm{CDCl}_{3}\right) \delta$ $8.49(\mathrm{~d}, J=8.9 \mathrm{~Hz}, 1 \mathrm{H}), 8.07(\mathrm{~d}, J=8.9 \mathrm{~Hz}, 1 \mathrm{H}), 7.94(\mathrm{~d}, J=8.2 \mathrm{~Hz}, 1 \mathrm{H}), 7.60(\mathrm{t}, J=$ $7.4 \mathrm{~Hz}, 1 \mathrm{H}), 7.44-7.36(\mathrm{~m}, 2 \mathrm{H}), 7.32-7.27(\mathrm{~m}, 3 \mathrm{H}), 7.23-7.17(\mathrm{~m}, 4 \mathrm{H}), 6.94(\mathrm{~d}, J$ $=7.3 \mathrm{~Hz}, 2 \mathrm{H}) ;{ }^{13} \mathrm{C} \mathrm{NMR}\left(100 \mathrm{MHz}, \mathrm{CDCl}_{3}\right) \delta 141.3,140.0,139.0,136.6,135.3$, 134.7, 133.4, 131.1, 129.1, 128.7, 128.6, 128.4, 128.0, 127.9, 127.6, 127.5, 127.2, 123.3; HRMS (CI-TOF) m/z: $[\mathrm{M}+\mathrm{H}]^{+}$calcd for $\mathrm{C}_{22} \mathrm{H}_{16} \mathrm{ClO}_{2} \mathrm{~S} 379.0554$, found 379.0563.<smiles>Cc1cccc(S(=O)(=O)c2ccc3ccccc3c2-c2ccc(Br)cc2)c1</smiles> 
White solid (52.2 mg, 73\%); mp 121-123 ${ }^{\circ} \mathrm{C} ;{ }^{1} \mathrm{H}$ NMR (400 MHz, $\left.\mathrm{CDCl}_{3}\right) \delta 8.52(\mathrm{~d}, J$ $=8.8 \mathrm{~Hz}, 1 \mathrm{H}), 8.07(\mathrm{~d}, J=8.9 \mathrm{~Hz}, 1 \mathrm{H}), 7.94(\mathrm{~d}, J=8.2 \mathrm{~Hz}, 1 \mathrm{H}), 7.62-7.55(\mathrm{~m}, 1 \mathrm{H})$, $7.43-7.34(\mathrm{~m}, 2 \mathrm{H}), 7.32-7.24(\mathrm{~m}, 3 \mathrm{H}), 7.23-7.18(\mathrm{~m}, 1 \mathrm{H}), 7.16-7.11(\mathrm{~m}, 2 \mathrm{H})$, 7.03 (s, 1H), $6.95-6.89$ (m, 2H), 2.23 (s, 3H); $\left.{ }^{13} \mathrm{C} \mathrm{NMR} \mathrm{(100} \mathrm{MHz,} \mathrm{CDCl}_{3}\right) \delta 141.2$, $138.4,137.2,135.2,134.7,133.5,133.3,131.1,128.5,128.3,128.19,128.18,127.9$, 127.7, 127.3, 127.1, 124.8, 123.5, 21.1; HRMS (CI-TOF) m/z: $[\mathrm{M}+\mathrm{H}]^{+}$calcd for $\mathrm{C}_{23} \mathrm{H}_{19} \mathrm{O}_{2} \mathrm{~S} 359.1100$, found 359.1108.<smiles>O=S(=O)(c1ccc2ccccc2c1)c1ccc2ccccc2c1-c1ccccc1</smiles>

5aa

Light yellow solid (40.8 mg, 52\%); mp 148-150 ${ }^{\circ} \mathrm{C} ;{ }^{1} \mathrm{H}$ NMR (400 $\left.\mathrm{MHz}, \mathrm{CDCl}_{3}\right) \delta$ $8.58(\mathrm{~d}, J=8.8 \mathrm{~Hz}, 1 \mathrm{H}), 8.09(\mathrm{~d}, J=8.9 \mathrm{~Hz}, 1 \mathrm{H}), 7.94(\mathrm{~d}, J=8.3 \mathrm{~Hz}, 1 \mathrm{H}), 7.81$ (d, $J$ $=8.1 \mathrm{~Hz}, 1 \mathrm{H}), 7.70(\mathrm{t}, J=9.6 \mathrm{~Hz}, 3 \mathrm{H}), 7.63-7.51(\mathrm{~m}, 3 \mathrm{H}), 7.39(\mathrm{dd}, J=8.6,1.8 \mathrm{~Hz}$, $1 \mathrm{H}), 7.37-7.24(\mathrm{~m}, 3 \mathrm{H}), 7.10(\mathrm{t}, J=7.7 \mathrm{~Hz}, 2 \mathrm{H}), 6.85(\mathrm{~d}, J=7.1 \mathrm{~Hz}, 2 \mathrm{H}) ;{ }^{13} \mathrm{C} \mathrm{NMR}$ $\left(100 \mathrm{MHz}, \mathrm{CDCl}_{3}\right) \delta 141.5,138.0,136.8,135.3,134.6,133.5,131.7,131.0,129.8$, $129.5,128.8,128.7,128.6,128.3,128.0,127.9$, 127.7, 127.6, 127.3, 127.2, 127.1, 123.6, 122.5; HRMS (CI-TOF) m/z: $[\mathrm{M}+\mathrm{H}]^{+}$calcd for $\mathrm{C}_{26} \mathrm{H}_{19} \mathrm{O}_{2} \mathrm{~S} 395.1100$, found 395.1107 . 


\section{X-ray structures of products 4a, 5a, and 5d}

X-ray structure of 4 a with thermal ellipsoids shown at the $30 \%$ probability level (CCDC1575978)

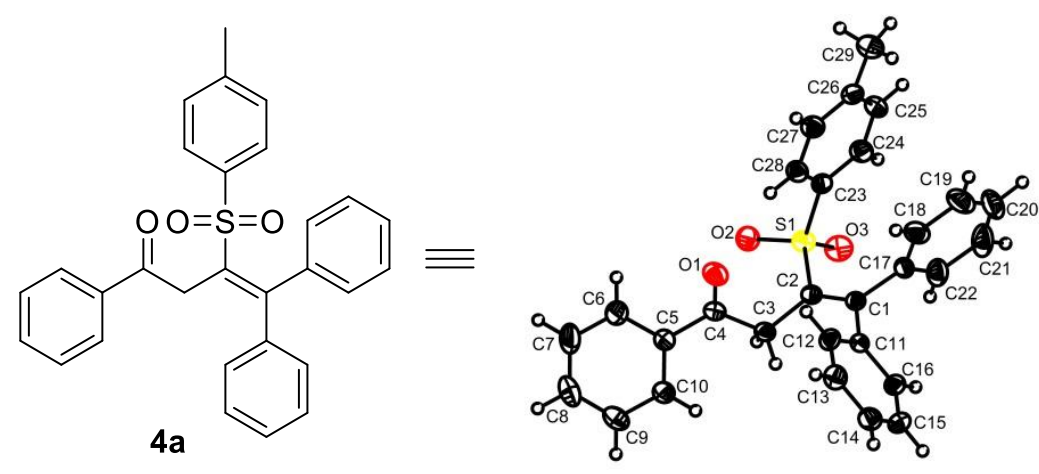

Crystal data and structure refinement for $\mathbf{4 a}$

Identification code

Empirical formula

Formula weight

Temperature

Wavelength

Crystal system

Space group

Unit cell dimensions

Volume

Z

Density (calculated)

Absorption coefficient

$\mathrm{F}(000)$

Crystal size

Theta range for data collection 4a

$\mathrm{C}_{29} \mathrm{H}_{24} \mathrm{O}_{3} \mathrm{~S}$

452.54

296(2) K

$0.71073 \AA$

Monoclinic

$\mathrm{P} 21 / \mathrm{n}$

$\mathrm{a}=12.1643(13) \AA \quad \alpha=90^{\circ}$.

$\mathrm{b}=14.1460(15) \AA \quad \beta=97.028(4)^{\circ}$.

$\mathrm{c}=14.0941(18) \AA \quad \gamma=90^{\circ}$.

2407.0(5) $\AA^{3}$

4

$1.249 \mathrm{Mg} / \mathrm{m}^{3}$

$0.163 \mathrm{~mm}^{-1}$

952

$0.450 \times 0.420 \times 0.380 \mathrm{~mm}^{3}$

2.218 to $27.541^{\circ}$. 
Index ranges

Reflections collected

Independent reflections

Completeness to theta $=25.242^{\circ}$

Absorption correction

Max. and min. transmission

Refinement method

Data / restraints / parameters

Goodness-of-fit on $\mathrm{F}^{2}$

Final R indices [I $>2 \operatorname{sigma}(\mathrm{I})]$

$\mathrm{R}$ indices (all data)

Extinction coefficient

Largest diff. peak and hole $-15<=\mathrm{h}<=15,-18<=\mathrm{k}<=16,-18<=\mathrm{l}<=18$

23500

$5512[\mathrm{R}(\mathrm{int})=0.0372]$

$99.7 \%$

Semi-empirical from equivalents

0.936 and 0.921

Full-matrix least-squares on $\mathrm{F}^{2}$

5512 / 0 / 299

1.058

$\mathrm{R} 1=0.0516, \mathrm{wR} 2=0.1193$

$\mathrm{R} 1=0.0867, \mathrm{wR} 2=0.1342$

$\mathrm{n} / \mathrm{a}$

0.195 and -0.438 e. $\AA^{-3}$

X-ray structure of 5 a with thermal ellipsoids shown at the $30 \%$ probability level (CCDC1575975)
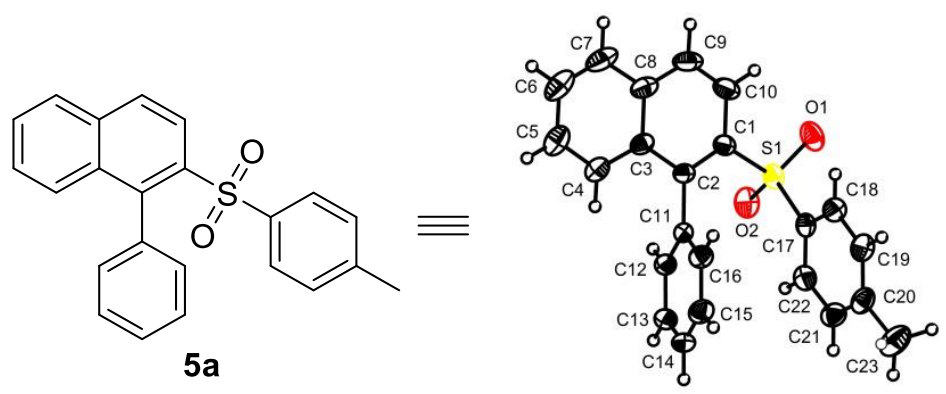

Crystal data and structure refinement for 5a

Identification code

Empirical formula

Formula weight

Temperature

Wavelength

Crystal system

Space group

Unit cell dimensions
$5 \mathbf{a}$

$\mathrm{C}_{23} \mathrm{H}_{18} \mathrm{O}_{2} \mathrm{~S}$

358.43

296(2) K

$0.71073 \AA$

Monoclinic

$\mathrm{P} 2{ }_{1} / \mathrm{c}$

$a=16.7547(9) \AA \quad \alpha=90^{\circ}$.

$\mathrm{b}=12.7100(6) \AA \quad \beta=96.098(2)^{\circ}$. 
Volume

Z

Density (calculated)

Absorption coefficient

$\mathrm{F}(000)$

Crystal size

Theta range for data collection

Index ranges

Reflections collected

Independent reflections

Completeness to theta $=25.242^{\circ}$

Absorption correction

Max. and min. transmission

Refinement method

Data / restraints / parameters

Goodness-of-fit on $\mathrm{F}^{2}$

Final R indices [I $>2 \operatorname{sigma}(\mathrm{I})]$

$\mathrm{R}$ indices (all data)

Extinction coefficient

Largest diff. peak and hole

$$
\mathrm{c}=8.7527(5) \AA \quad \gamma=90^{\circ} .
$$$$
1853.36(17) \AA^{3}
$$

4

$1.285 \mathrm{Mg} / \mathrm{m}^{3}$

$0.188 \mathrm{~mm}^{-1}$

752

$0.420 \times 0.380 \times 0.350 \mathrm{~mm}^{3}$

2.924 to $27.521^{\circ}$.

$-21<=\mathrm{h}<=18,-13<=\mathrm{k}<=16,-11<=\mathrm{l}<=11$

17488

$4206[\mathrm{R}$ (int) $=0.0304]$

$99.3 \%$

Semi-empirical from equivalents

0.932 and 0.916

Full-matrix least-squares on $\mathrm{F}^{2}$

4206 / 0 / 237

1.033

$\mathrm{R} 1=0.0446, \mathrm{wR} 2=0.1051$

$\mathrm{R} 1=0.0737, \mathrm{wR} 2=0.1170$

$0.016(3)$

0.204 and -0.309 e. $\AA^{-3}$

X-ray structure of $5 \mathrm{~d}$ with thermal ellipsoids shown at the $30 \%$ probability level (CCDC1575977)

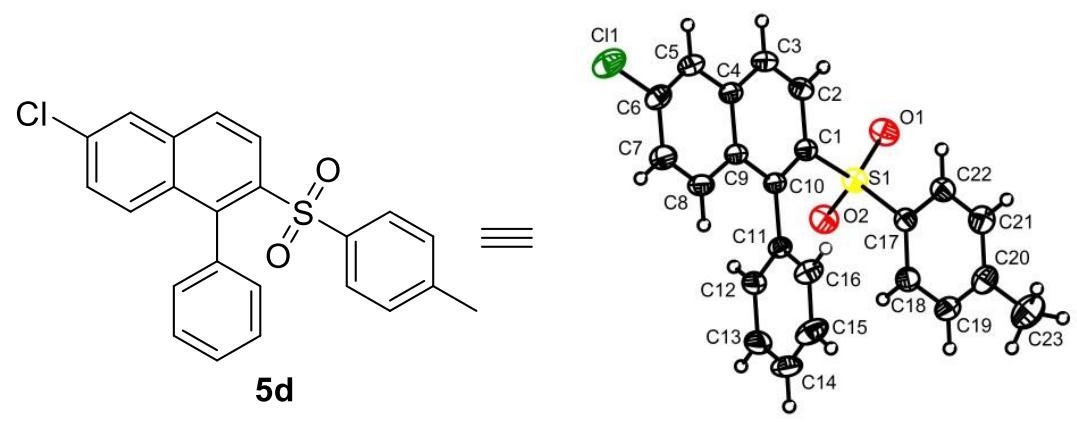




\section{Crystal data and structure refinement for $5 d$}

Identification code

Empirical formula

Formula weight

Temperature

Wavelength

Crystal system

Space group

Unit cell dimensions

Volume

Z

Density (calculated)

Absorption coefficient

$\mathrm{F}(000)$

Crystal size

Theta range for data collection

Index ranges

Reflections collected

Independent reflections

Completeness to theta $=25.242^{\circ}$

Absorption correction

Max. and min. transmission

Refinement method

Data / restraints / parameters

Goodness-of-fit on $\mathrm{F}^{2}$

Final R indices [I $>2 \operatorname{sigma}(\mathrm{I})]$

$\mathrm{R}$ indices (all data)

Extinction coefficient

Largest diff. peak and hole 5d

$\mathrm{C}_{23} \mathrm{H}_{17} \mathrm{ClO}_{2} \mathrm{~S}$

392.88

296(2) K

$0.71073 \AA$

Orthorhombic

Pbca

$\begin{array}{ll}\mathrm{a}=15.8989(6) \AA & \alpha=90^{\circ} . \\ \mathrm{b}=13.8847(5) \AA & \beta=90^{\circ} . \\ \mathrm{c}=17.7068(7) \AA & \gamma=90^{\circ} .\end{array}$

3908.8(3) $\AA^{3}$

8

$1.335 \mathrm{Mg} / \mathrm{m}^{3}$

$0.317 \mathrm{~mm}^{-1}$

1632

$0.320 \times 0.280 \times 0.250 \mathrm{~mm}^{3}$

2.262 to $27.537^{\circ}$.

$-20<=\mathrm{h}<=20,-17<=\mathrm{k}<=18,-18<=\mathrm{l}<=23$

33576

$4489[\mathrm{R}(\mathrm{int})=0.0415]$

$99.8 \%$

Semi-empirical from equivalents

0.922 and 0.901

Full-matrix least-squares on $\mathrm{F}^{2}$

4489 / 0 / 245

1.038

$\mathrm{R} 1=0.0481, \mathrm{wR} 2=0.1132$

$\mathrm{R} 1=0.0818, \mathrm{wR} 2=0.1277$

$\mathrm{n} / \mathrm{a}$

0.307 and -0.398 e. $\AA^{-3}$ 


\section{References}

(1) Gao, P.; Shen, Y.-W.; Fang, R.; Hao, X.-H.; Qiu, Z.-H.; Yang, F.; Yan, X.-B.; Wang, Q.; Gong, X.-J.; Liu, X.-Y.; Liang, Y.-M. Angew. Chem., Int. Ed. 2014, 53, 7629.

(2) Liu, S.; Zhao, J.; Kaminsky, L.; Wilson, R. J.; Marino, N.; Clark, D. A. Org. Lett. 2014, 16, 4456.

(3) Shen, R.; Yang, J.; Zhu, S.; Chen, C.; Wu, L. Adv. Synth. Catal. 2015, 357, 1259.

(4) Hirashita, T.; Suzuki, Y.; Tsuji, H.; Sato, Y.; Naito, K.; Araki, S. Eur. J. Org. Chem. 2012, 2012, 5668.

(5) Deguest, G.; Bischoff, L.; Fruit, C.; Marsais, F. Synth. Commun. 2008, 38, 841.

(6) (a) Jones, C. M.; Burkitt, M. J. J. Chem. Soc., Perkin Trans. II 2002, 2044. (b) Chen, Y.-R.; Mason, R. P. Biochem. J. 2002, 365, 461. (c) Takahashi, Y.; Ichimori, K.; Okano, M.; Goto, H. J. Clin. Biochem. Nutr. 2015, 56, 105. 
The determination of the structures of $4 \mathrm{~m}$ and $4 \mathrm{n}$

HMBC experiment of $\mathbf{4 m}$

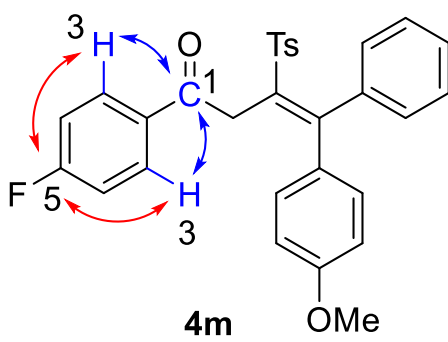

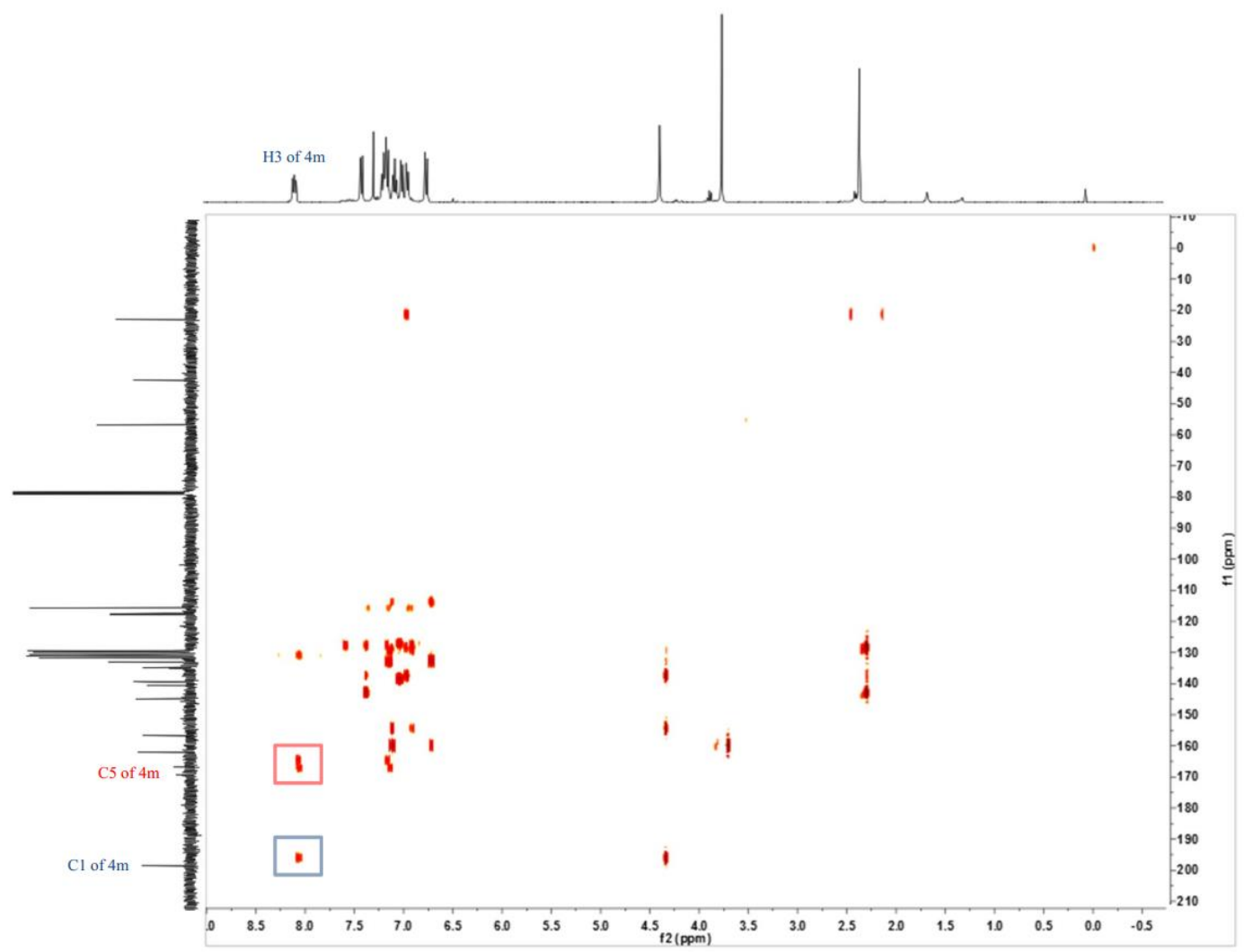


HMBC experiment of $\mathbf{4 n}$

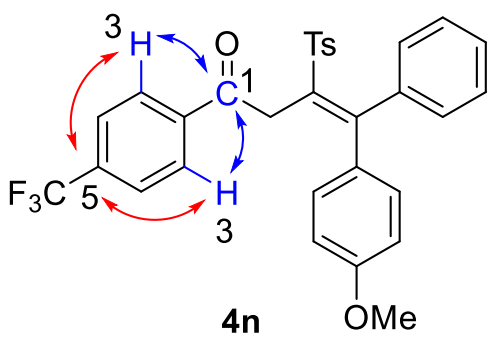

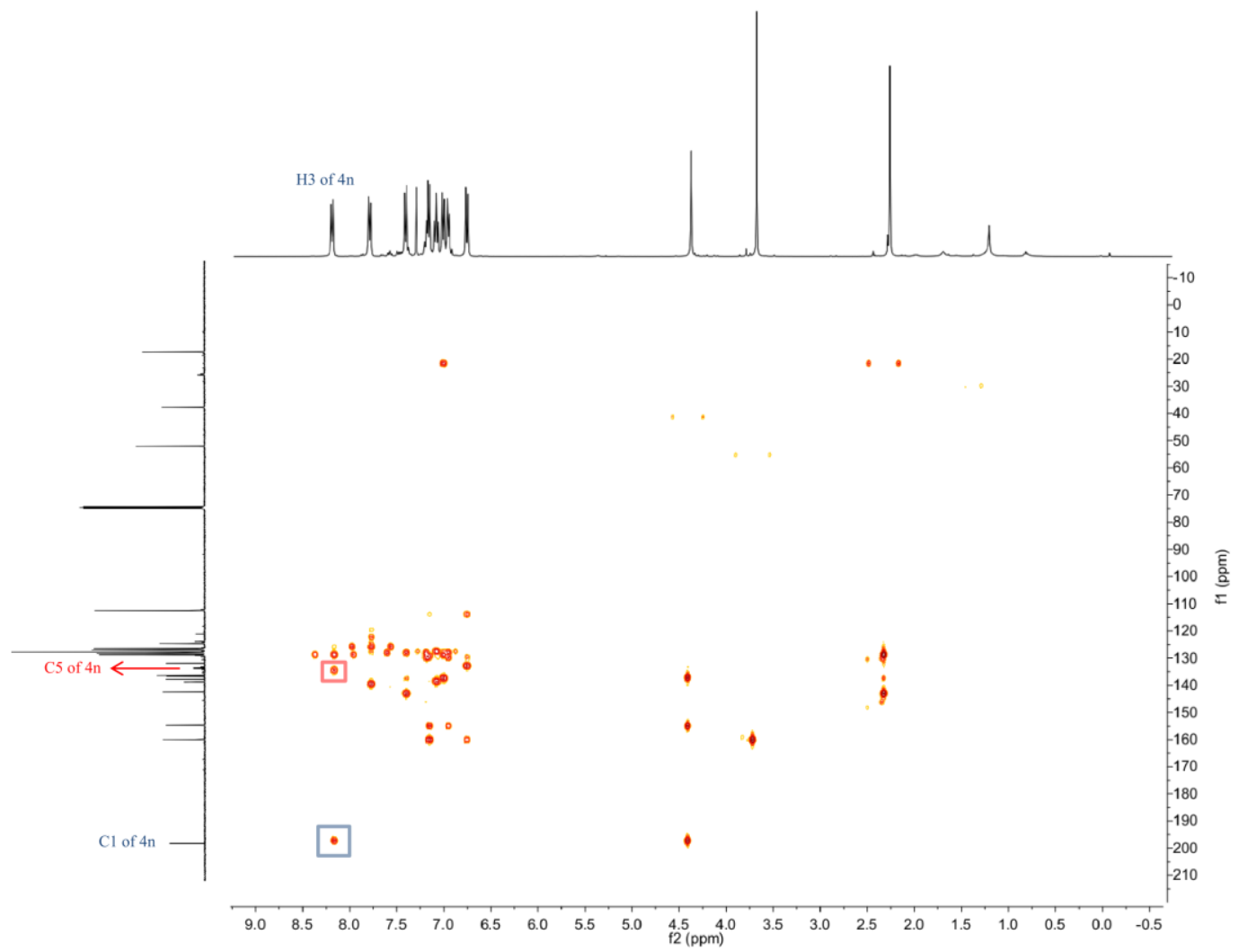



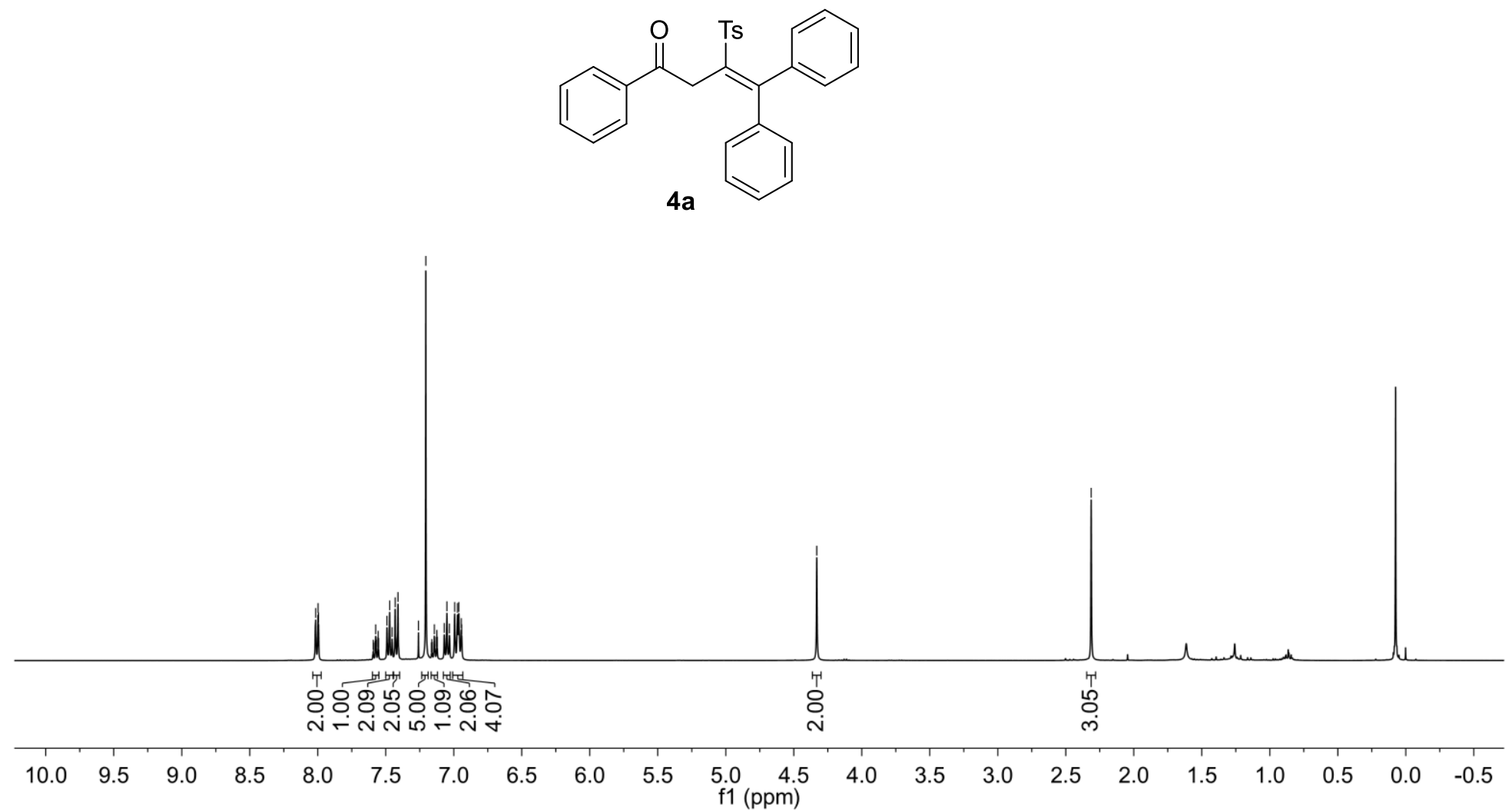

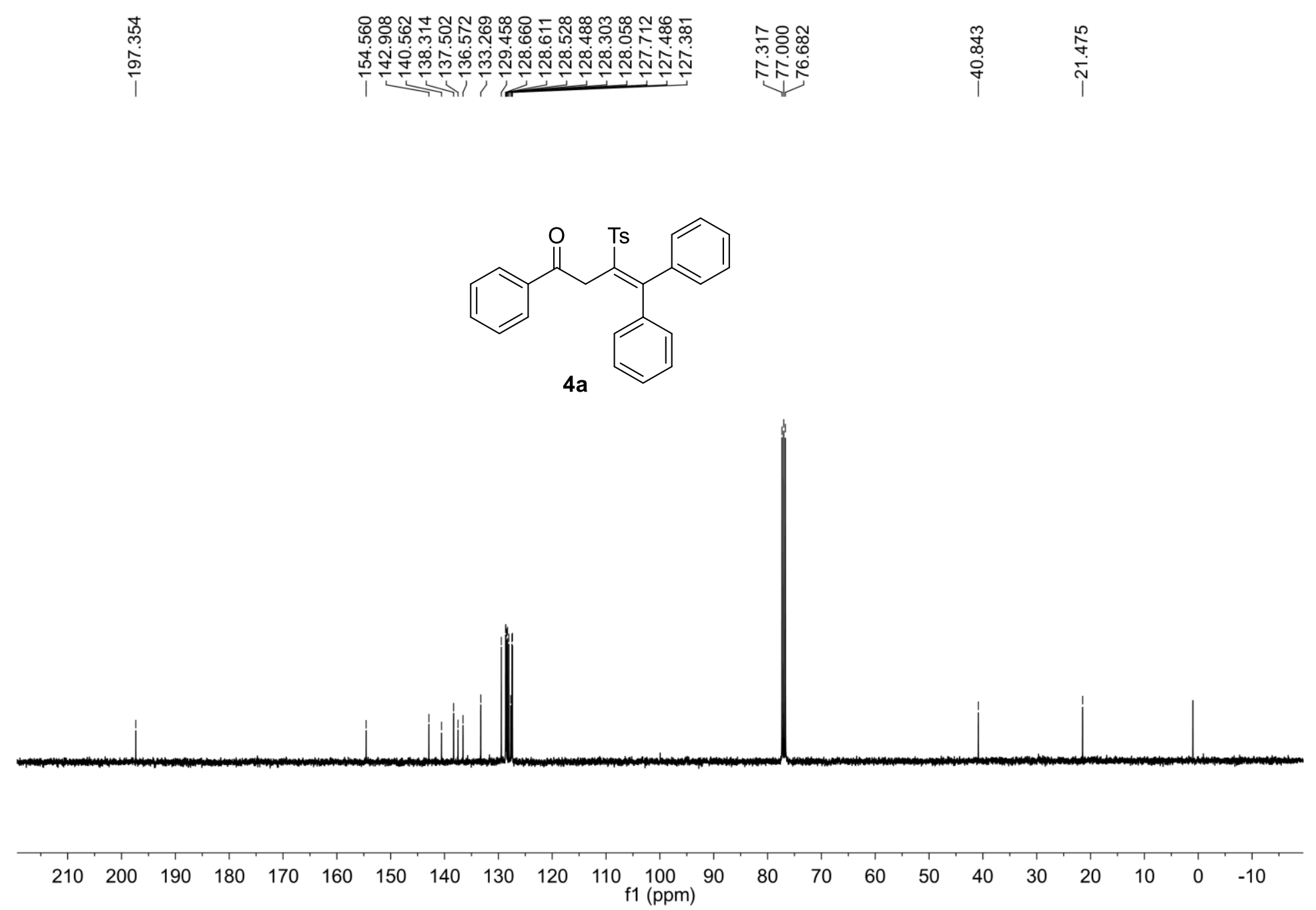


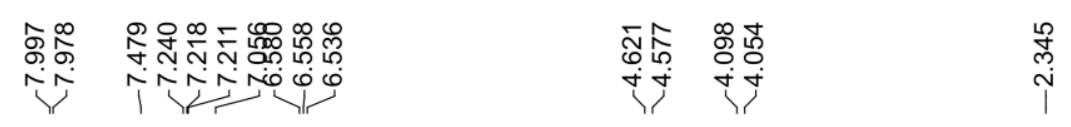
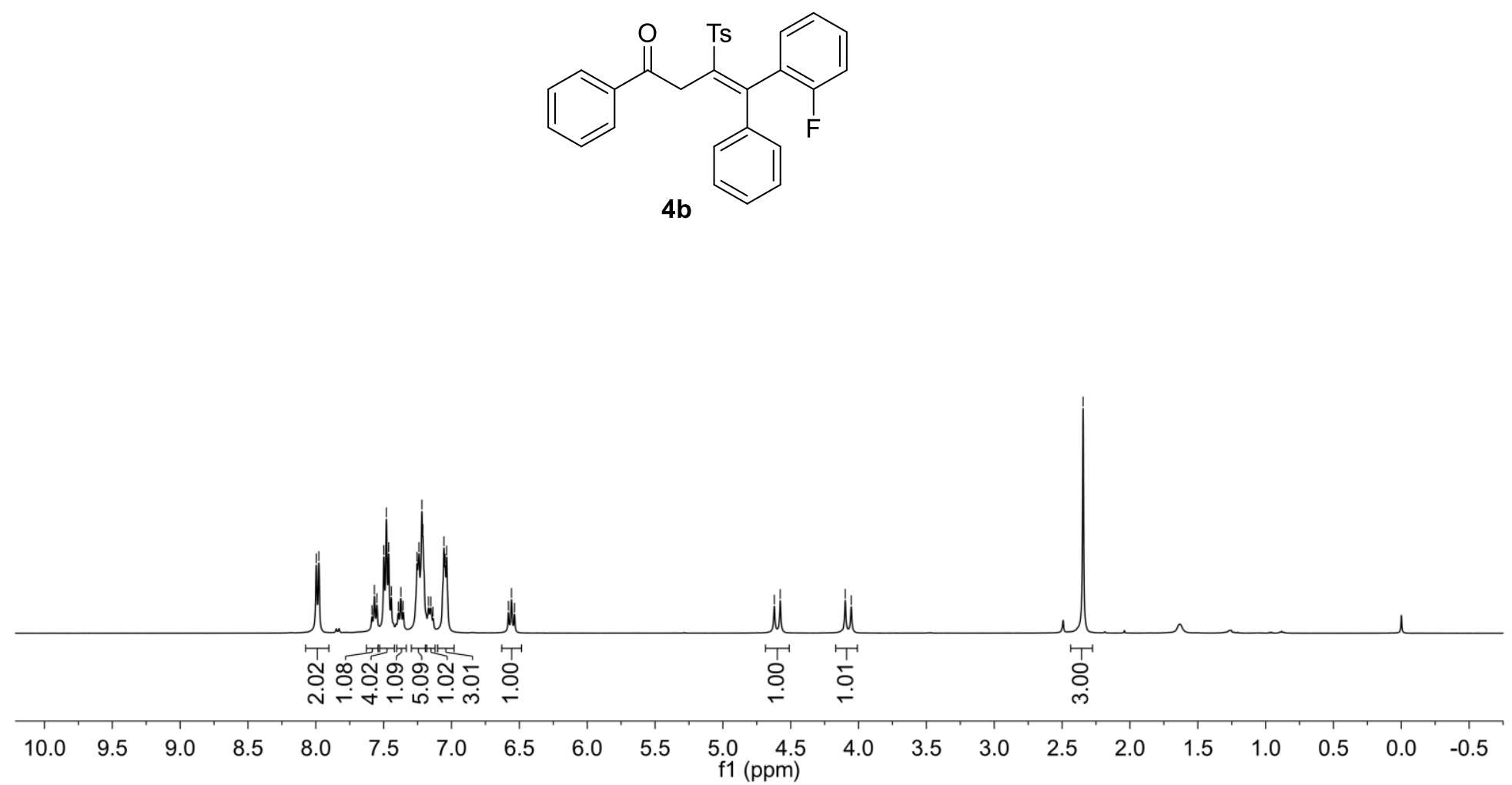

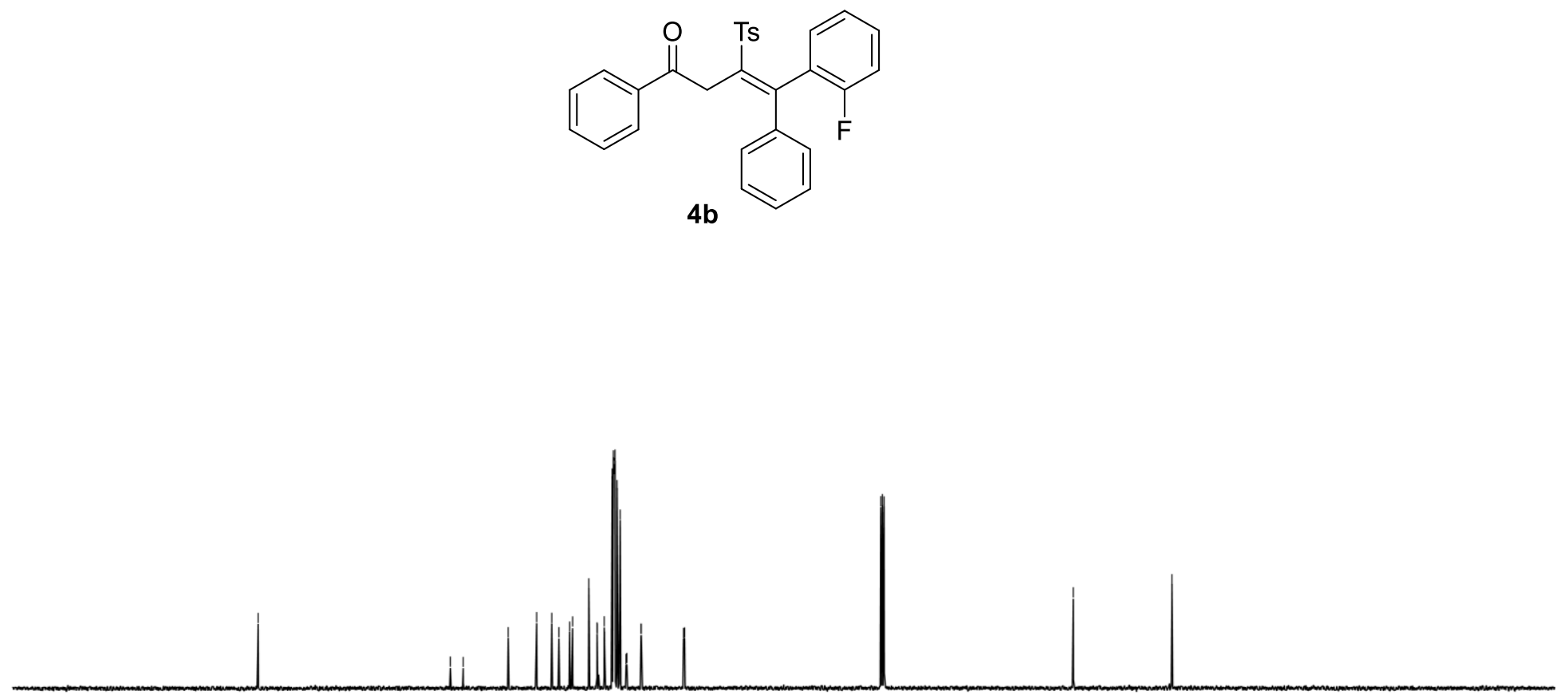

$\begin{array}{llllllllllllllllllllllllllllllllll}240 & 230 & 220 & 210 & 200 & 190 & 180 & 170 & 160 & 150 & 140 & 130 & 120 & 110 & 100 & 90 & 80 & 70 & 60 & 50 & 40 & 30 & 20 & 10 & 0 & -10 & -20 & -30 & -40 & -50\end{array}$ f1 (ppm) 

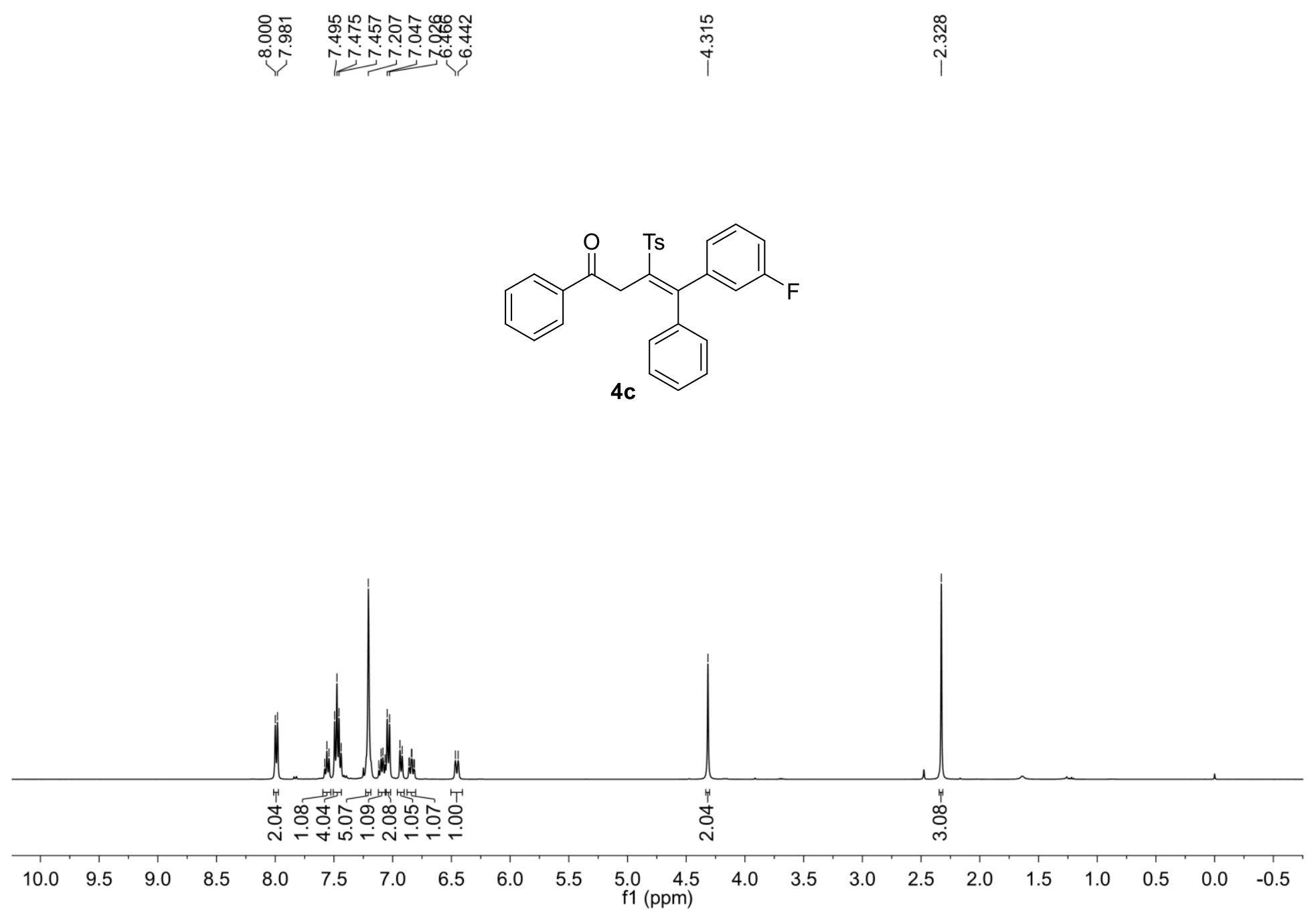

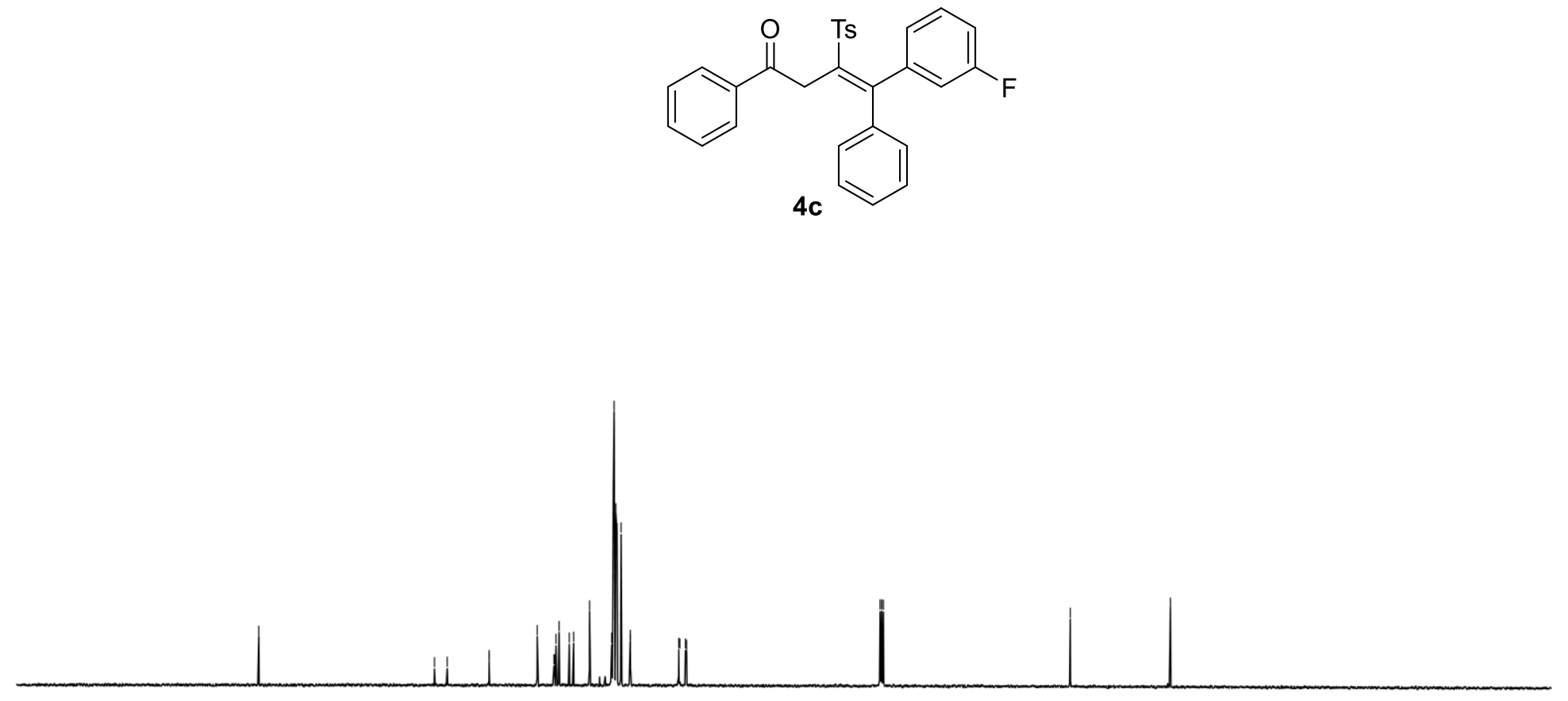

\begin{tabular}{llllllllllllllllllllllllllllllll}
\hline 240 & 230 & 220 & 210 & 200 & 190 & 180 & 170 & 160 & 150 & 140 & 130 & 120 & 110 & 100 & 90 & 80 & 70 & 60 & 50 & 40 & 30 & 20 & 10 & 0 & -10 & -20 & -30 & -40 & -5(
\end{tabular} f1 (ppm) 

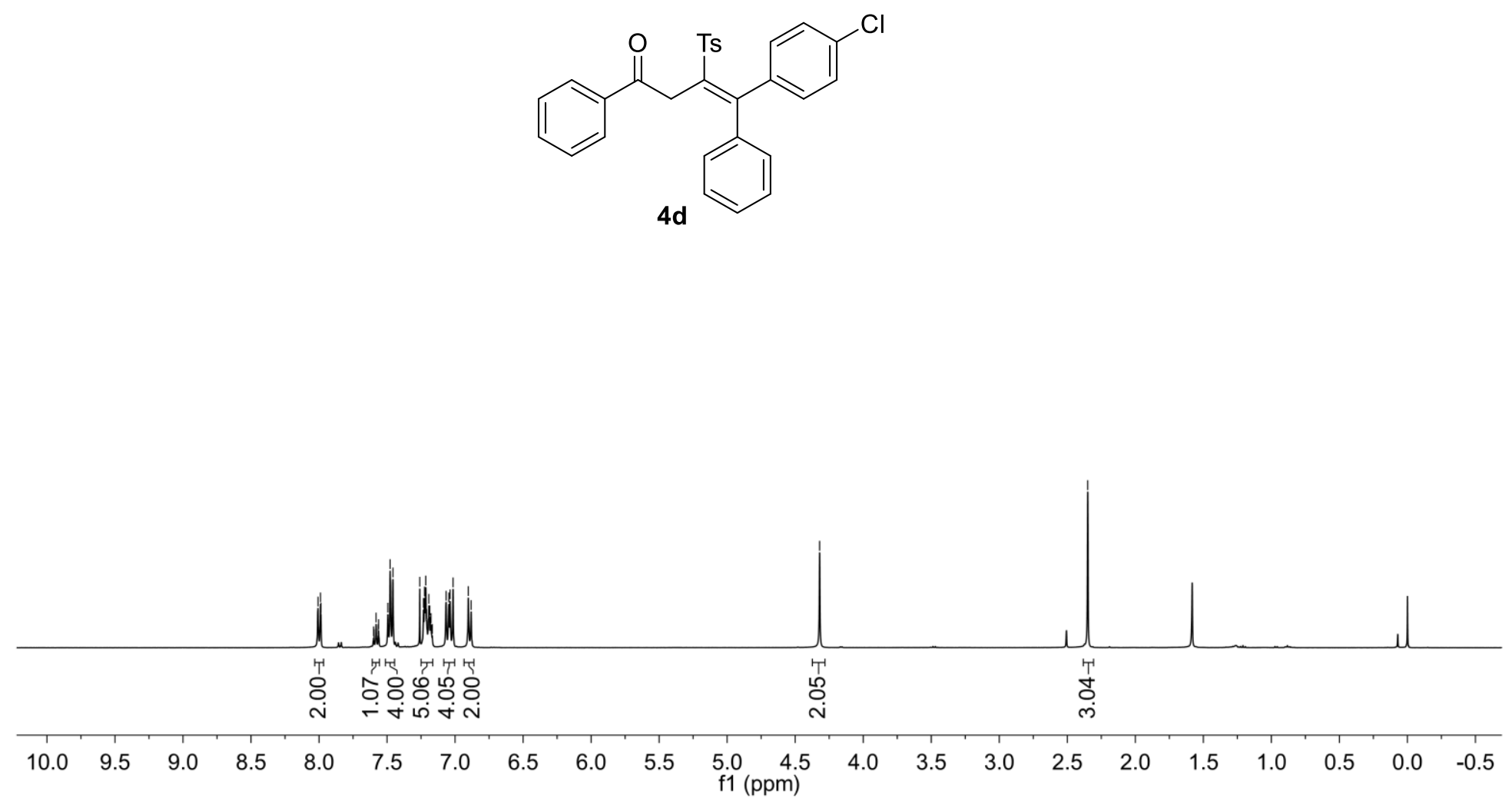

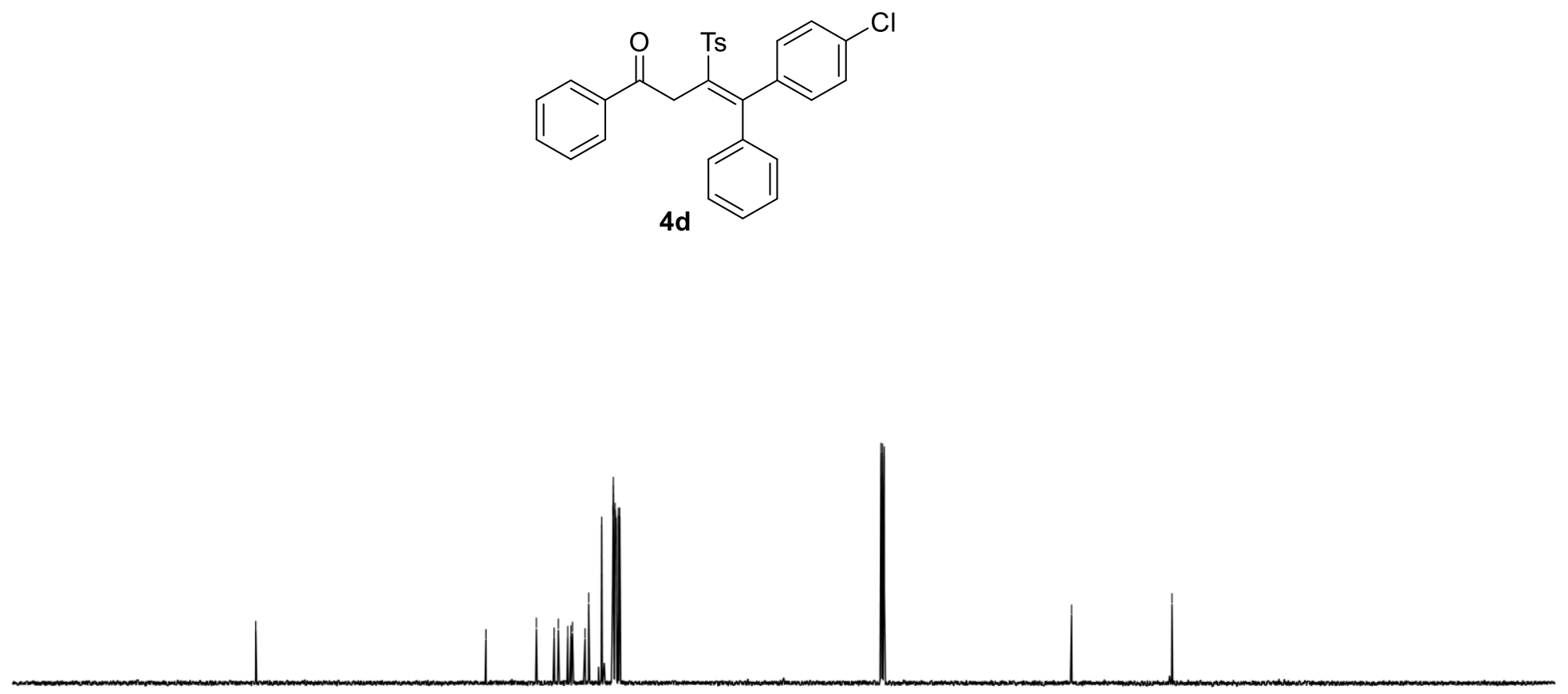

$\begin{array}{lllllllllllllllllllllllllllllllll}240 & 230 & 220 & 210 & 200 & 190 & 180 & 170 & 160 & 150 & 140 & 130 & 120 & 110 & 100 & 90 & 80 & 70 & 60 & 50 & 40 & 30 & 20 & 10 & 0 & -10 & -20 & -30 & -40 & -50\end{array}$ f1 (ppm) 

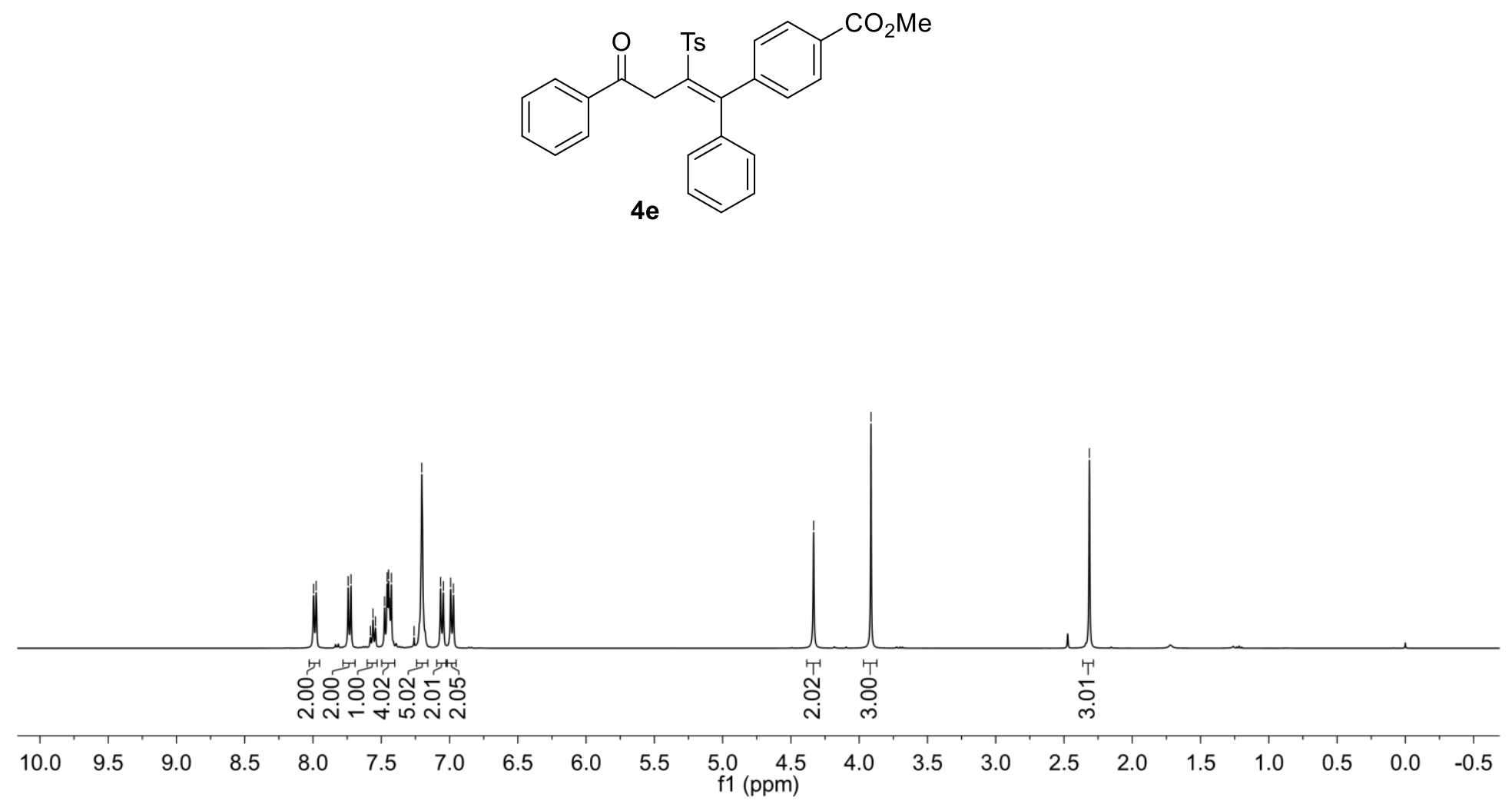

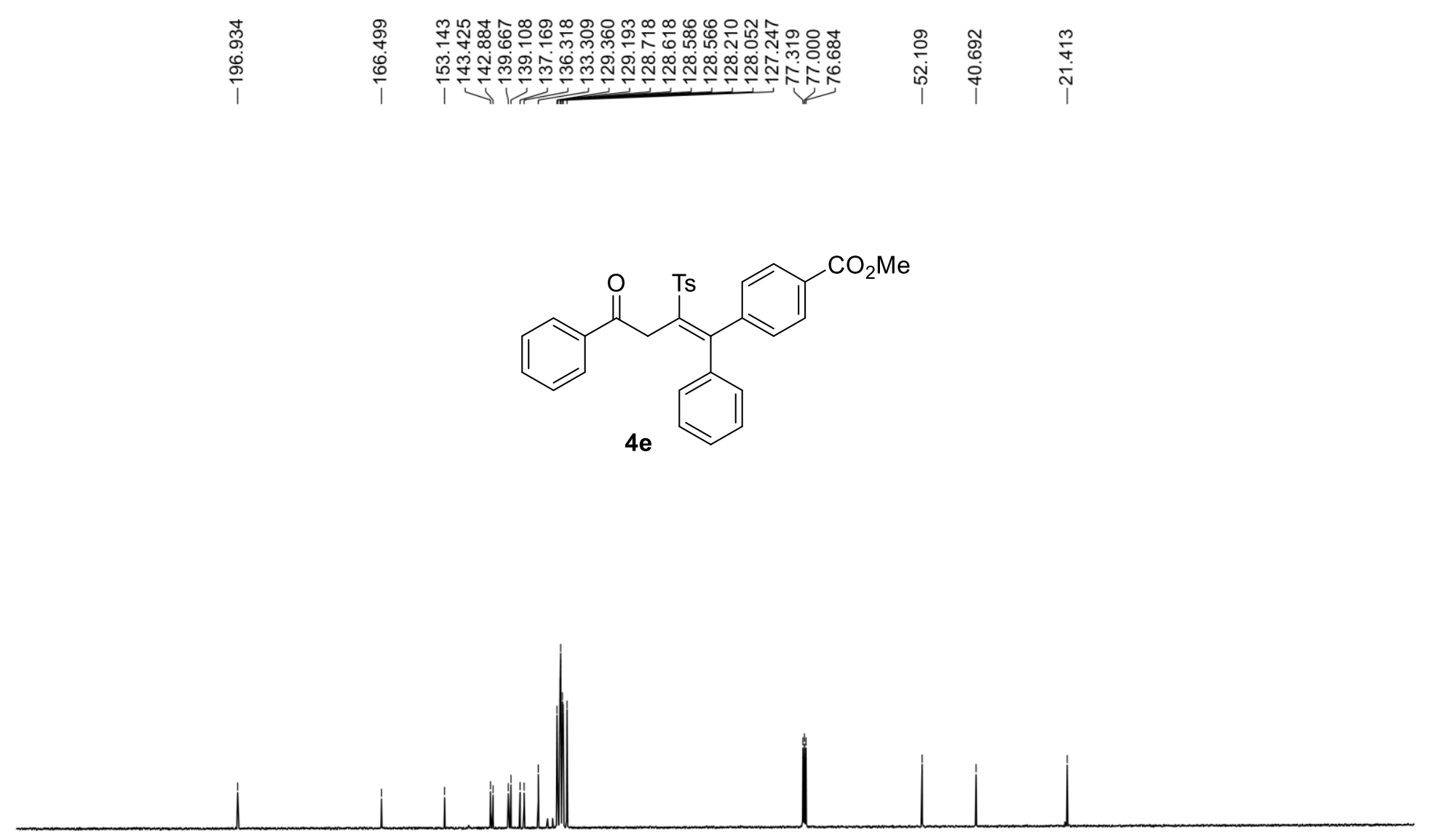

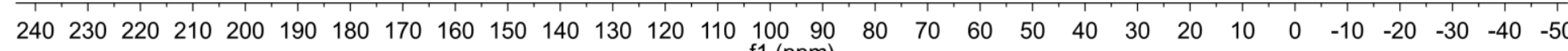
f1 (ppm) 

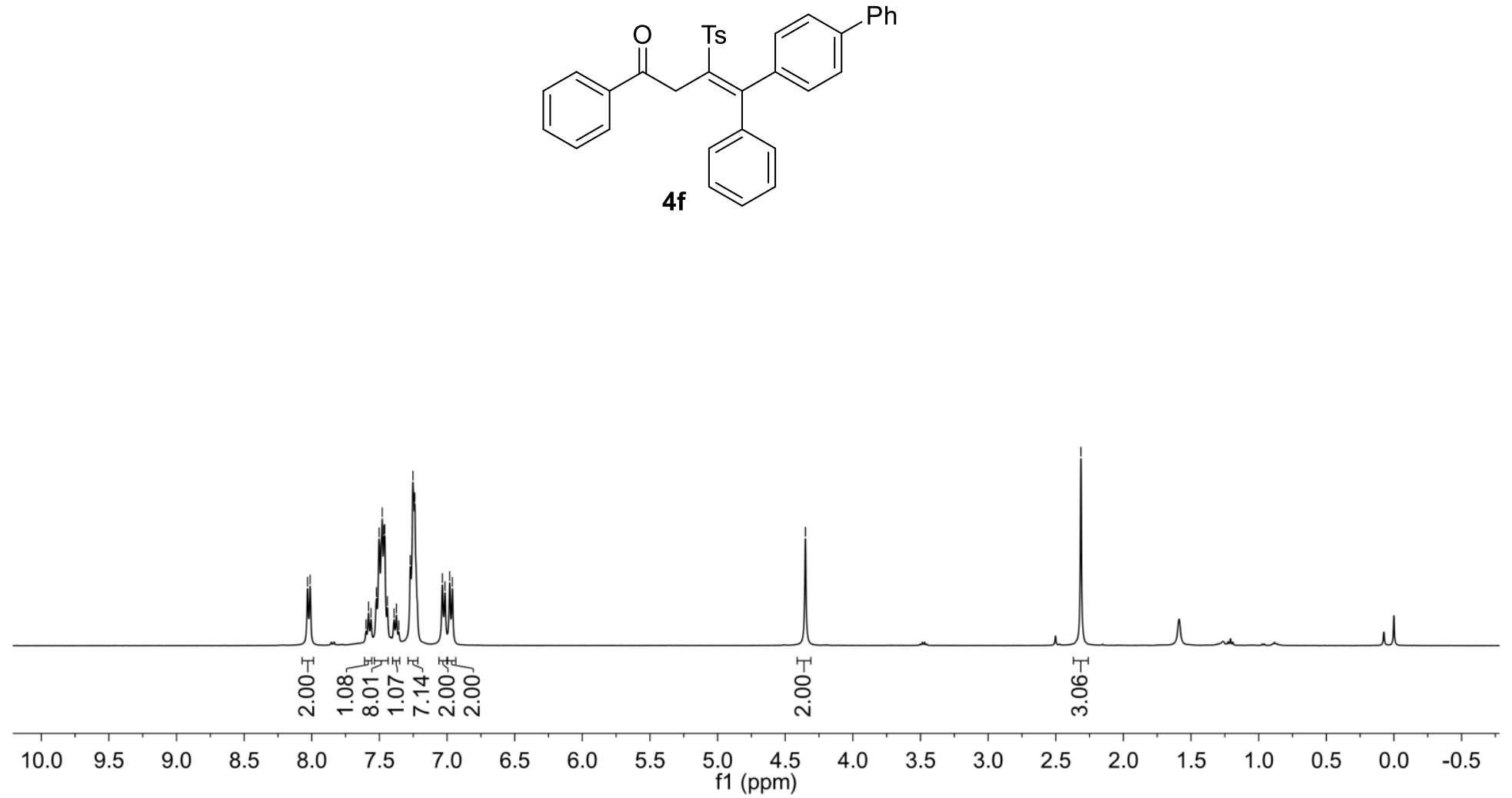

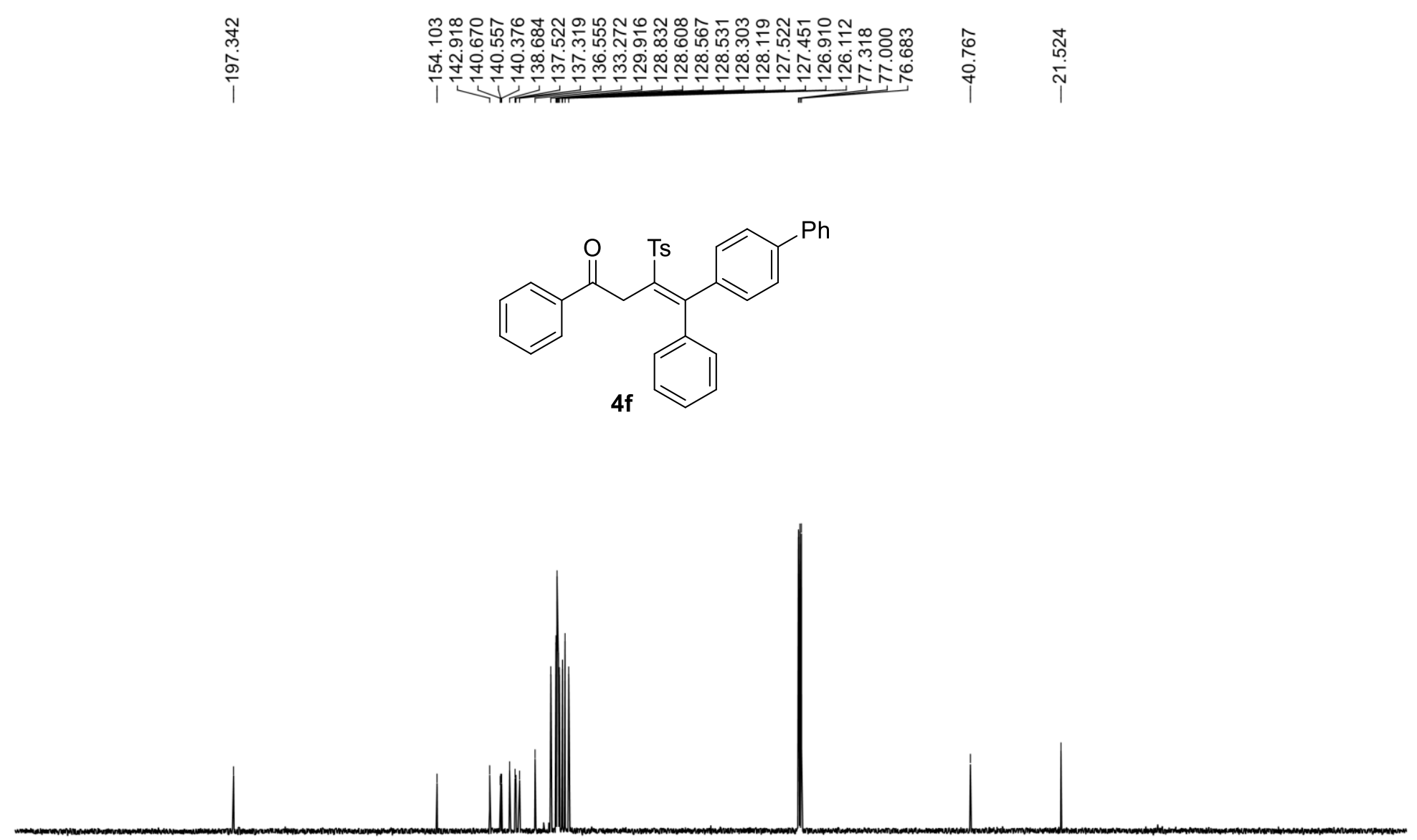

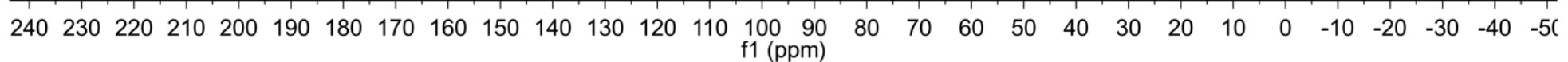



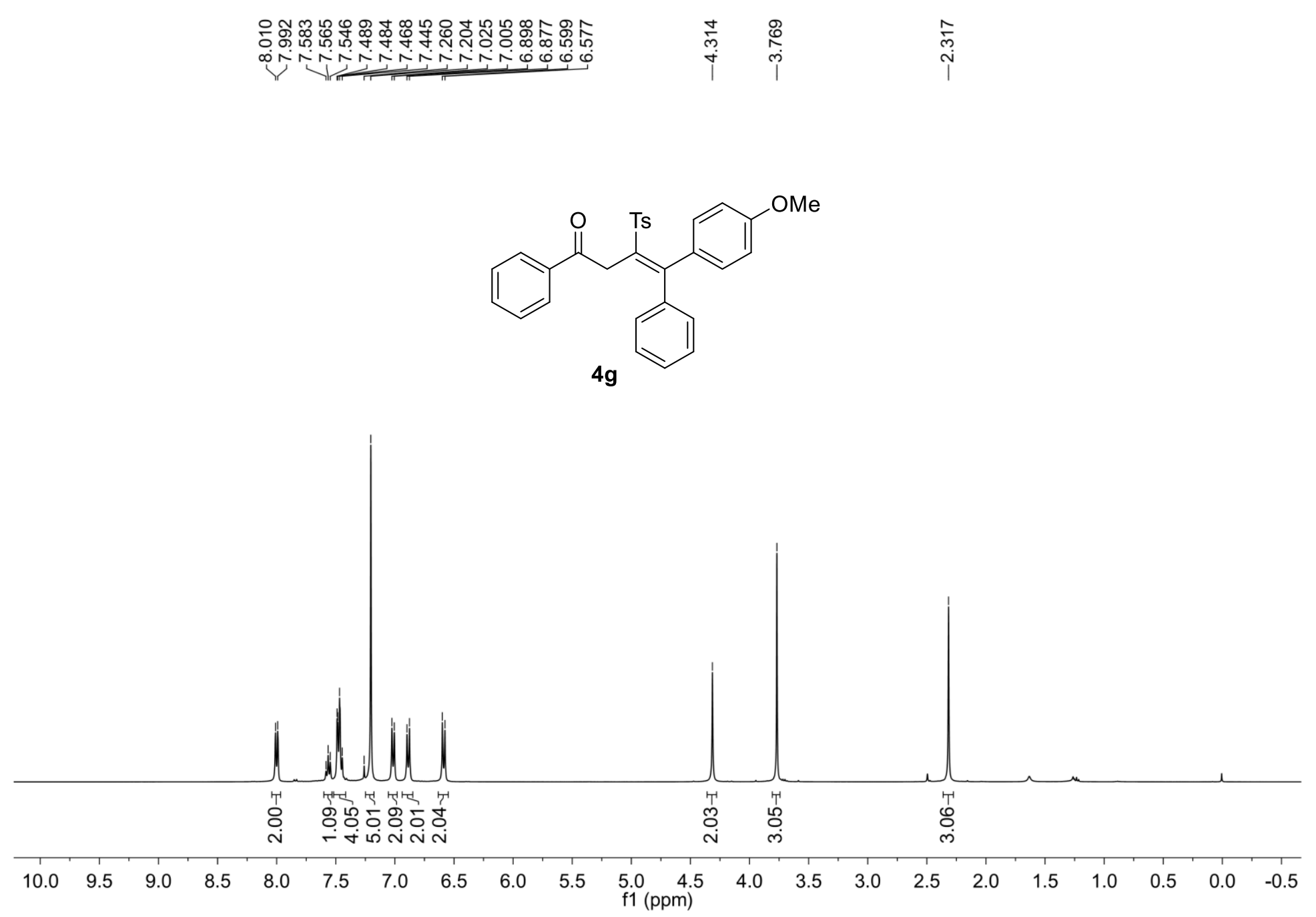

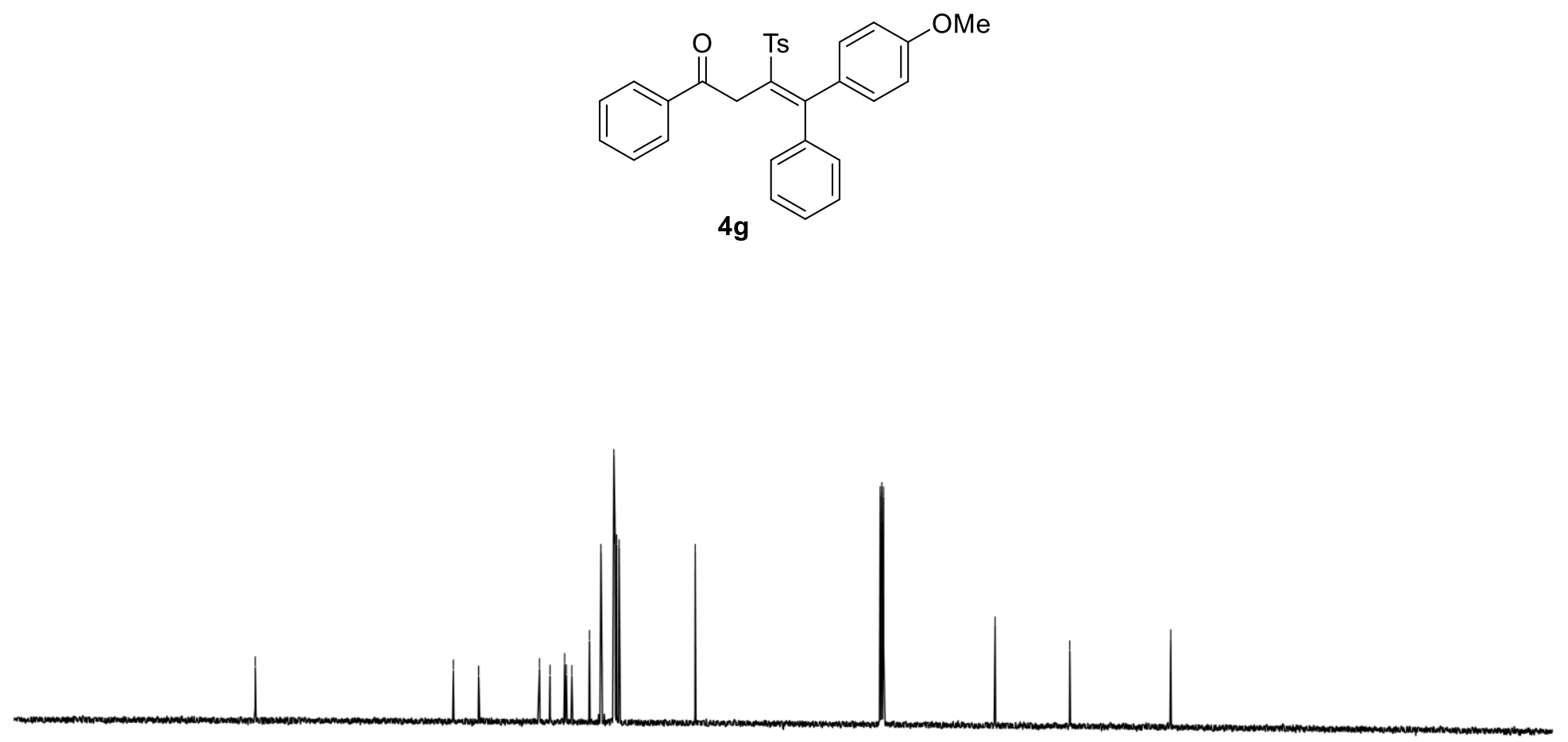

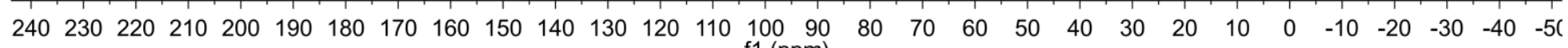
f1 (ppm) 
至

$\stackrel{\text { N }}{\text { N }}$
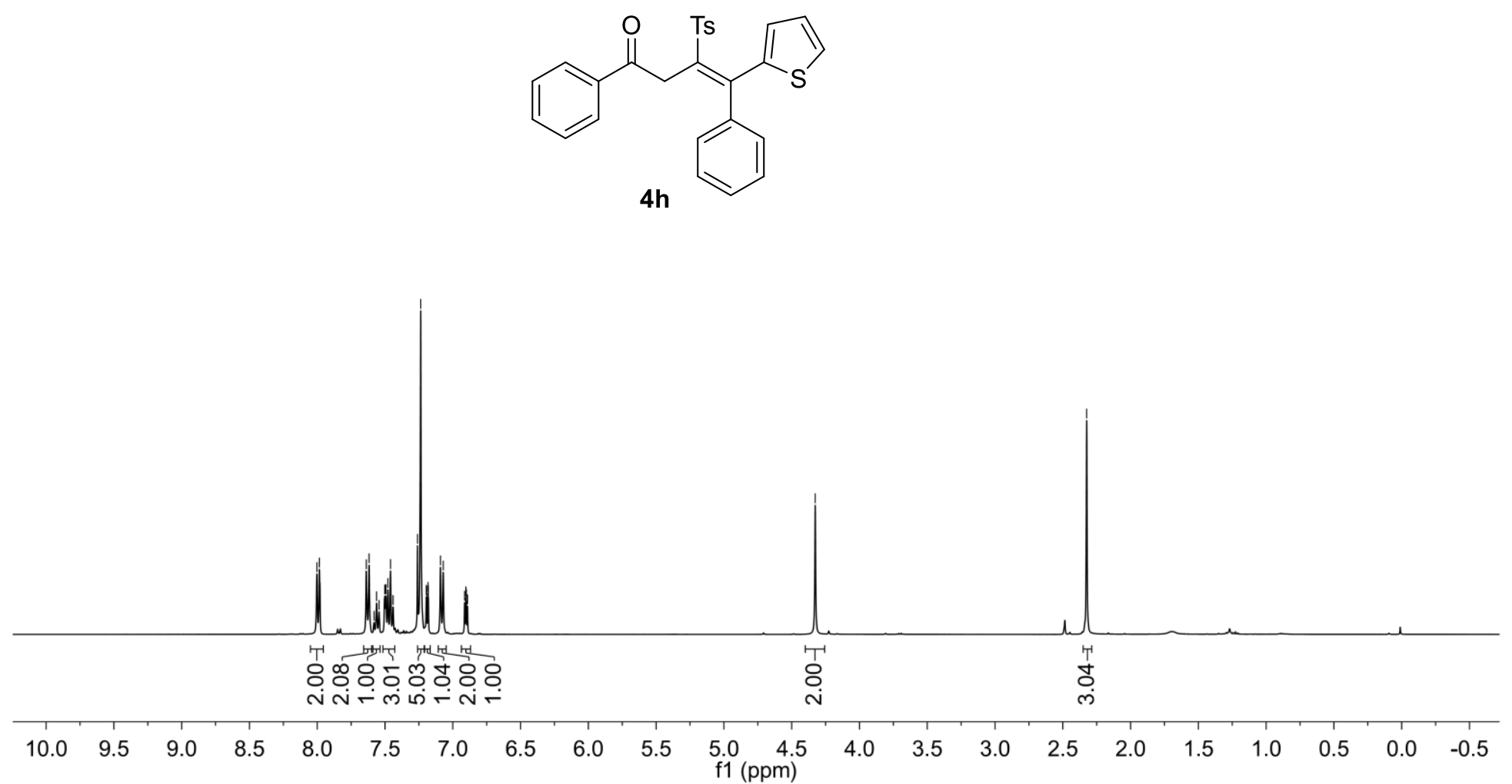

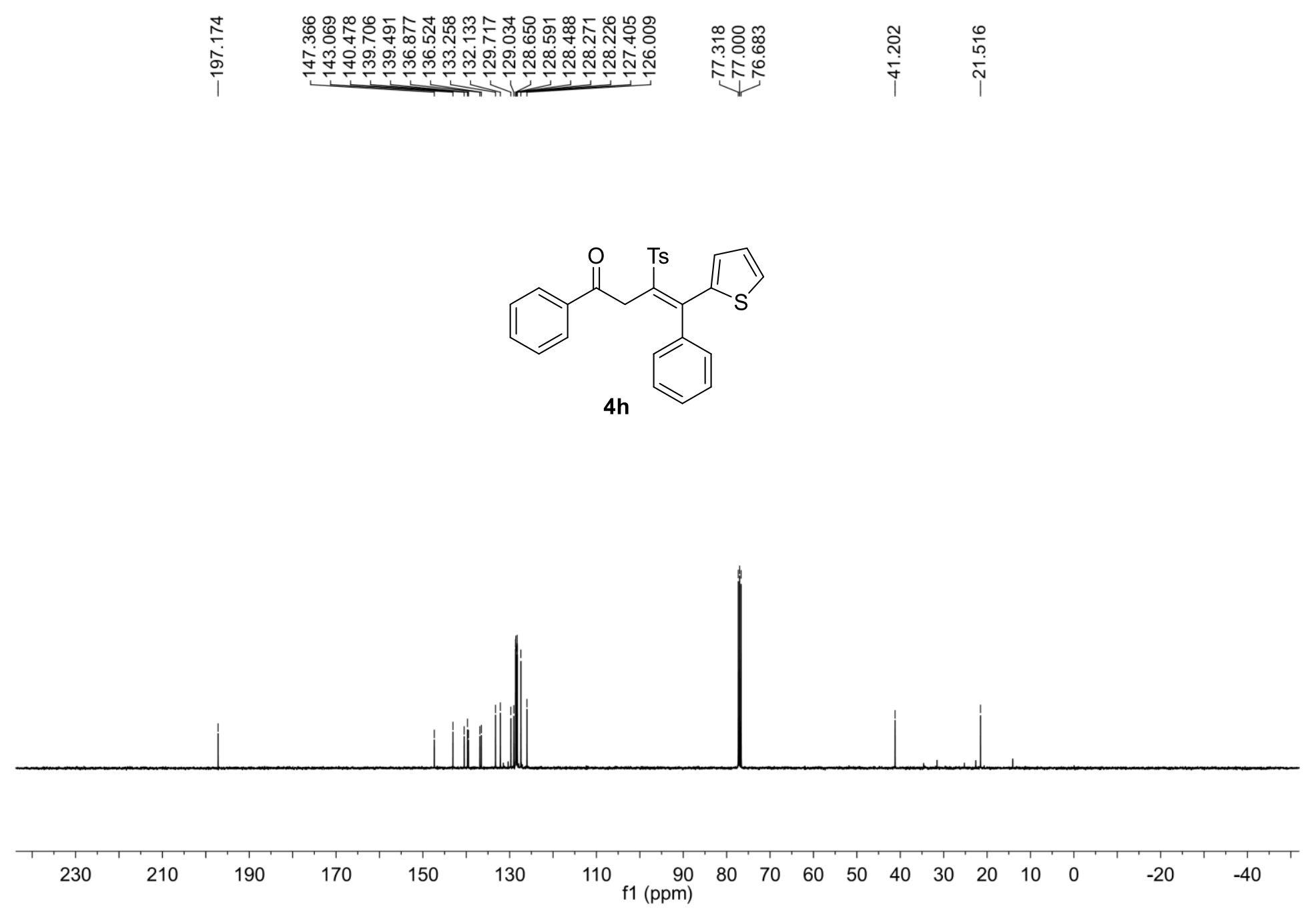

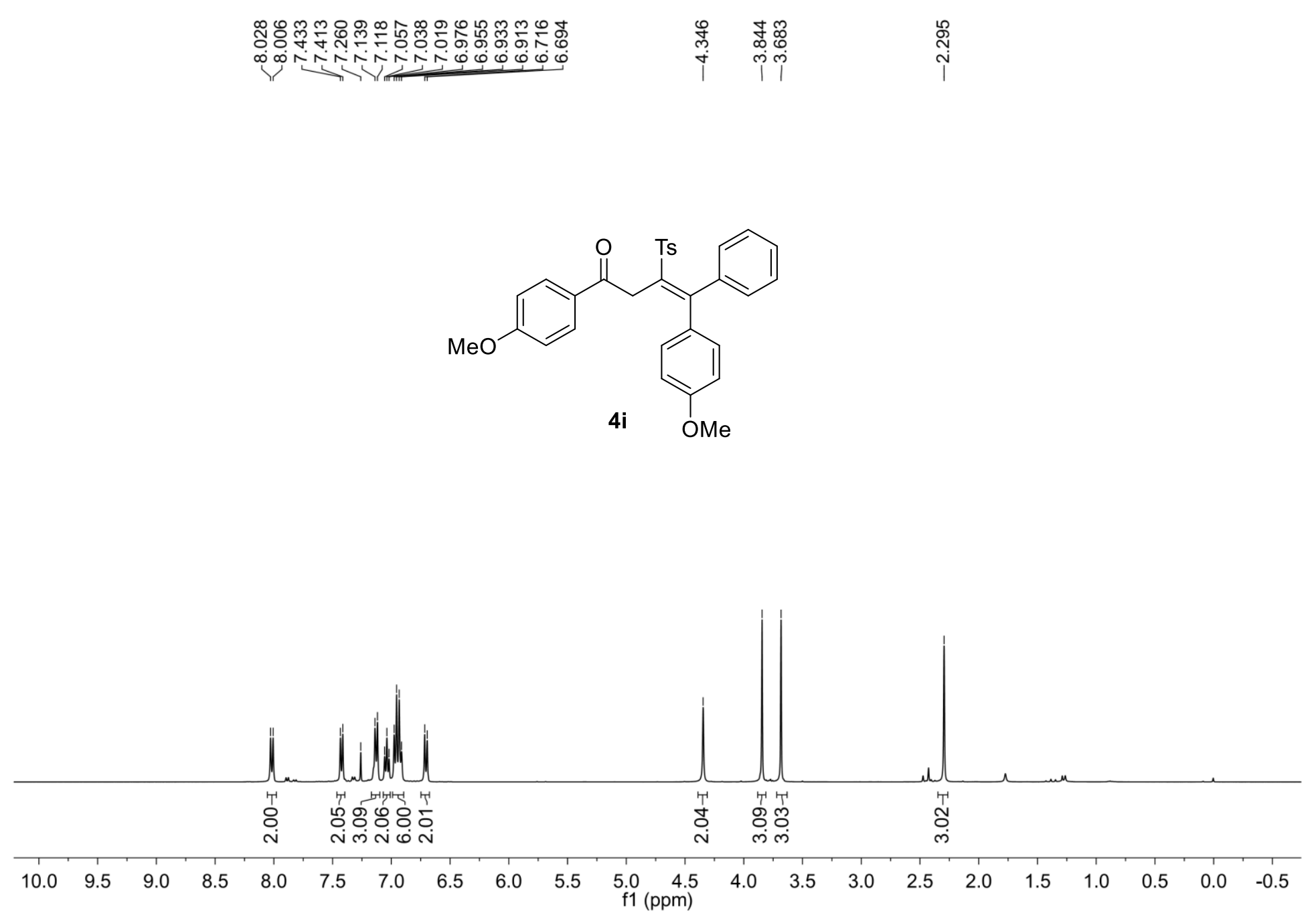


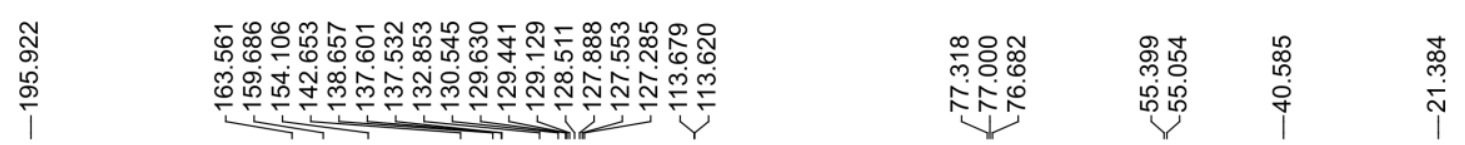
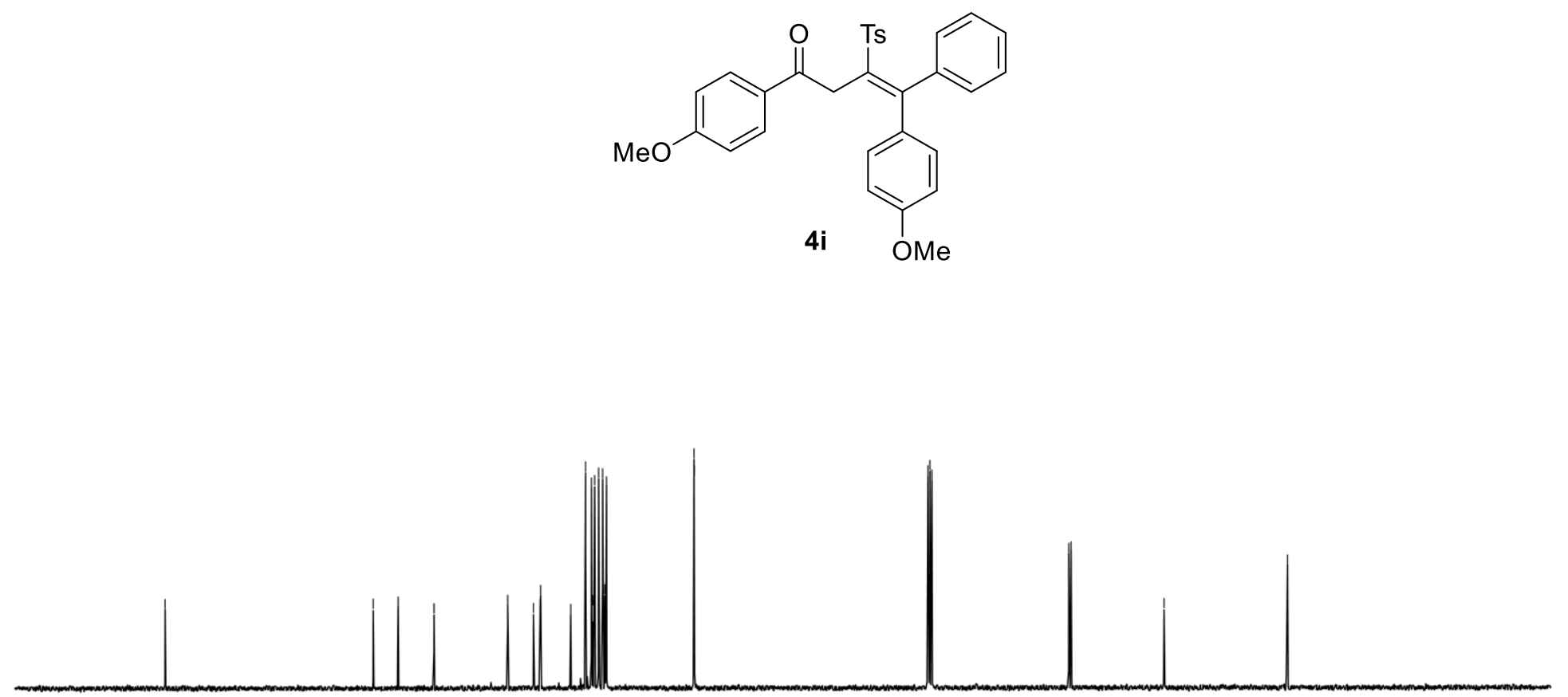

$\begin{array}{llllllllllllllllllllll}210 & 200 & 190 & 180 & 170 & 160 & 150 & 140 & 130 & 120 & 110 \begin{array}{c}100 \\ \mathrm{f} 1(\mathrm{ppm})\end{array} & 90 & 80 & 70 & 60 & 50 & 40 & 30 & 20 & 10 & 0 & -10\end{array}$ 


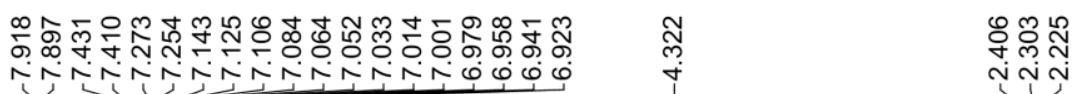
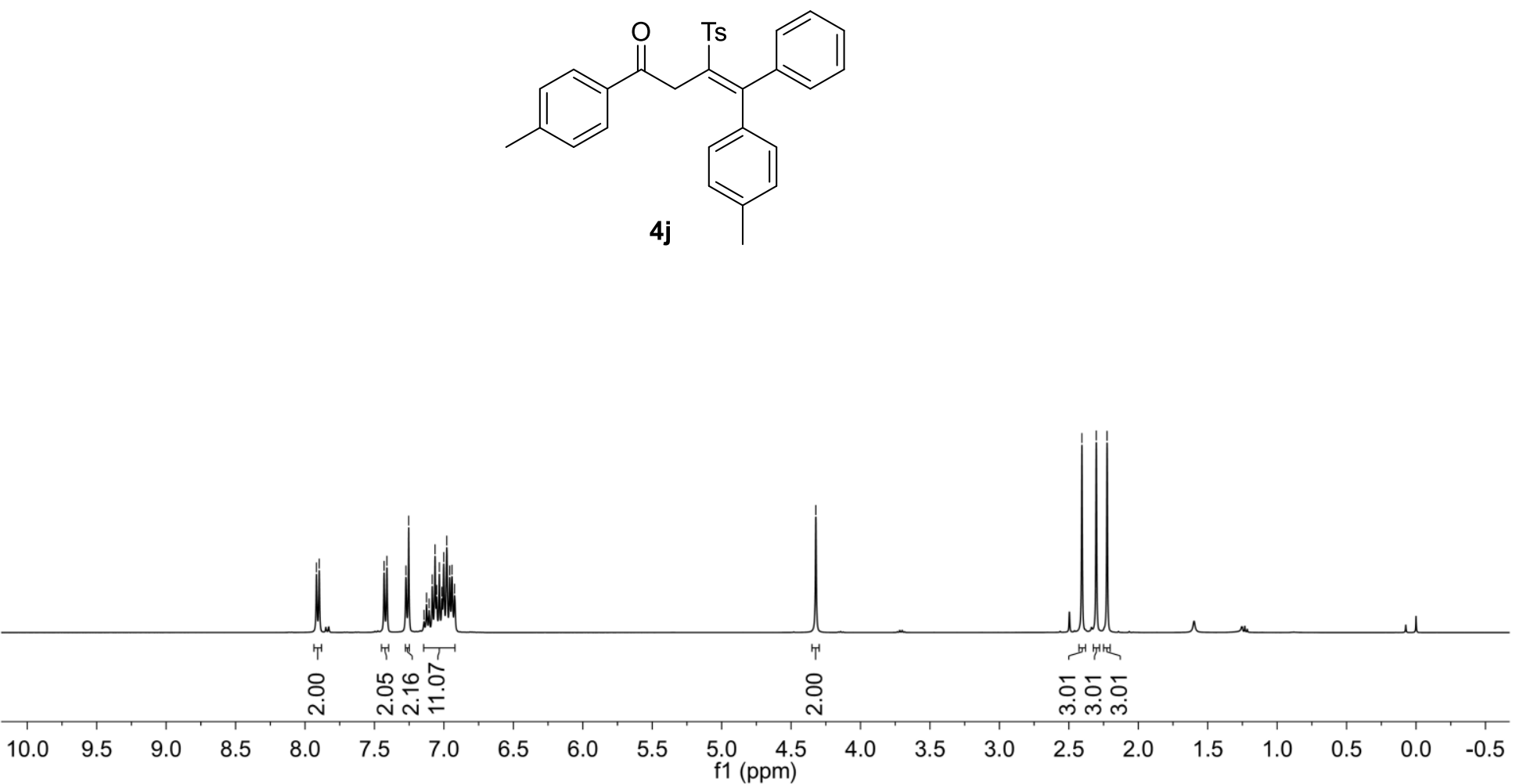

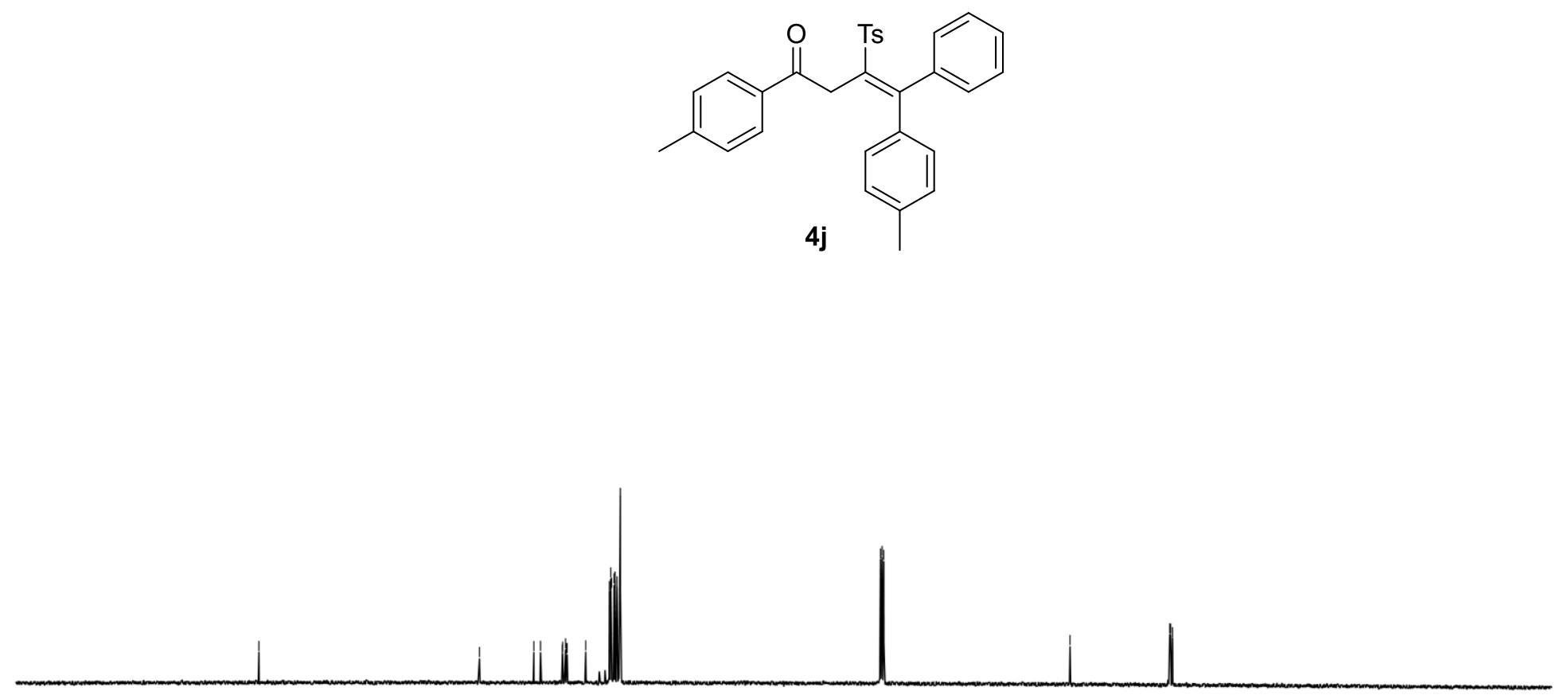

$\begin{array}{llllllllllllllllllllllllllllllll}240 & 230 & 220 & 210 & 200 & 190 & 180 & 170 & 160 & 150 & 140 & 130 & 120 & 110 & 100 & 90 & 80 & 70 & 60 & 50 & 40 & 30 & 20 & 10 & 0 & -10 & -20 & -30 & -40 & -5(\end{array}$ f1 (ppm) 


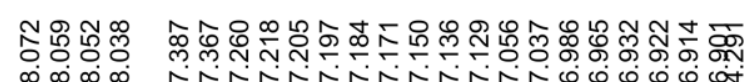

क्ष
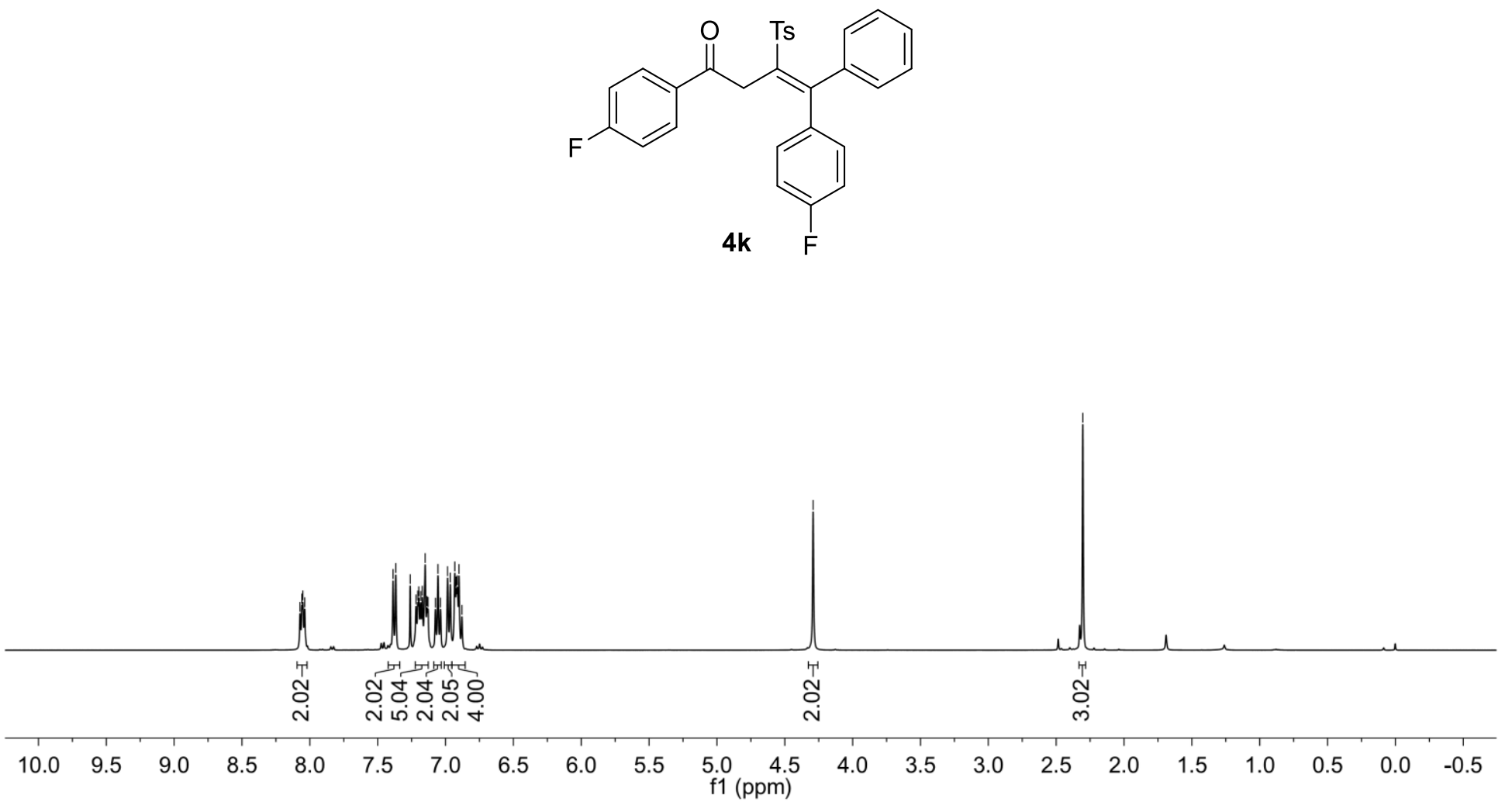


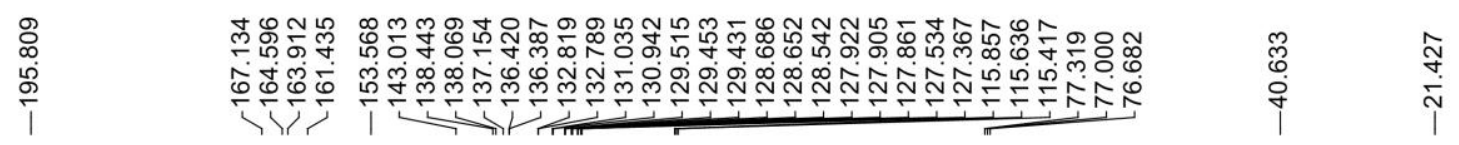
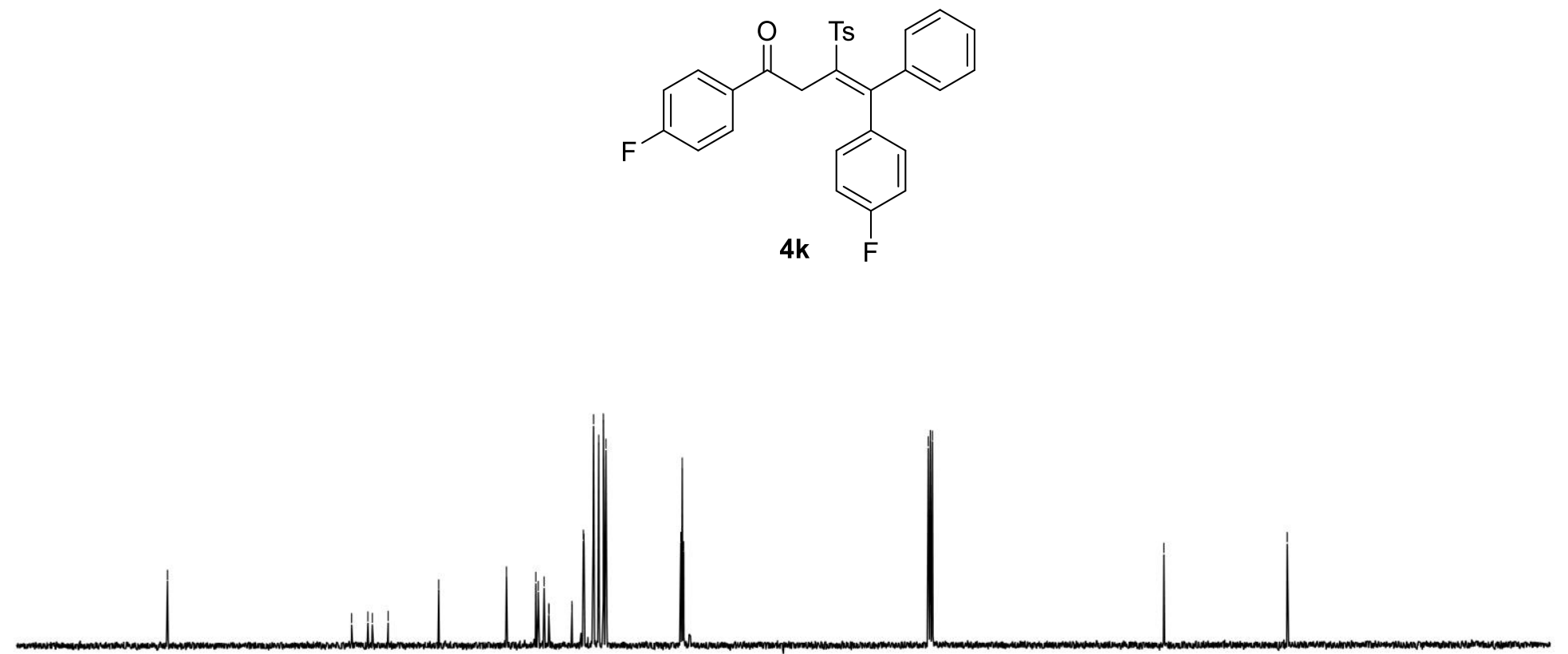

$\begin{array}{llllllllllllllllllllll}210 & 200 & 190 & 180 & 170 & 160 & 150 & 140 & 130 & 120 & 110 \begin{array}{c}100 \\ \mathrm{f} 1(\mathrm{ppm})\end{array} & 90 & 80 & 70 & 60 & 50 & 40 & 30 & 20 & 10 & 0 & -10\end{array}$ 


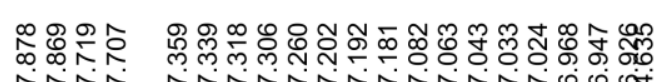

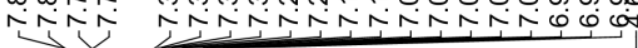

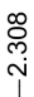
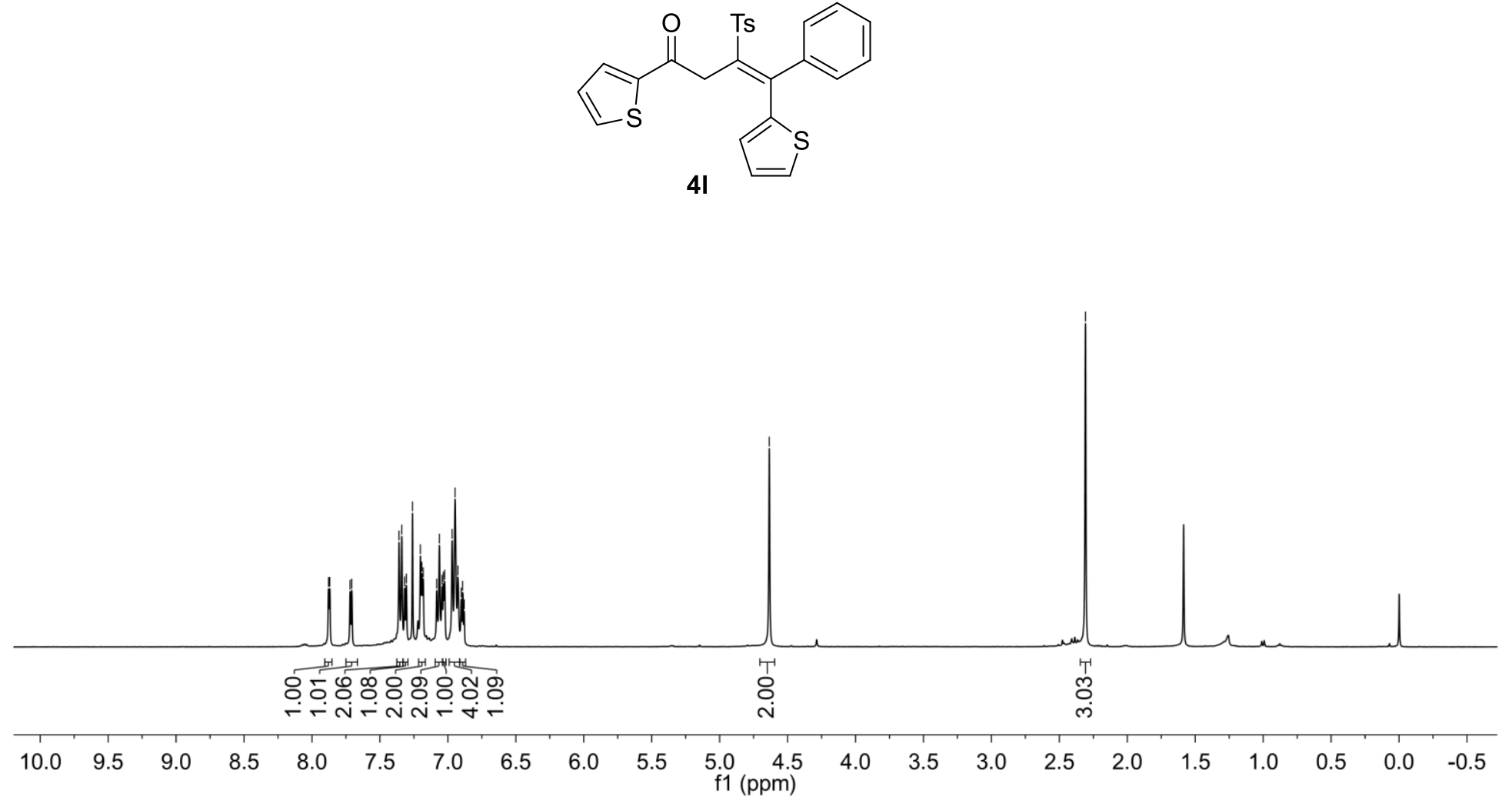


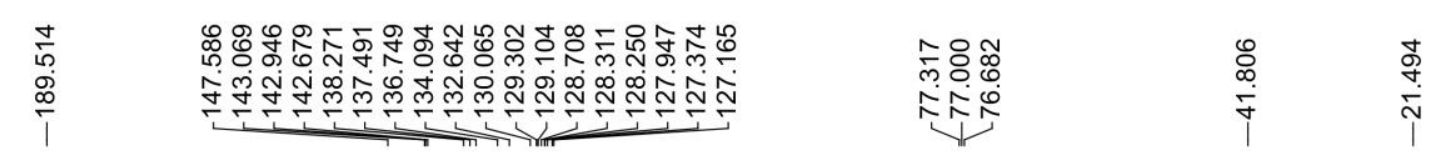
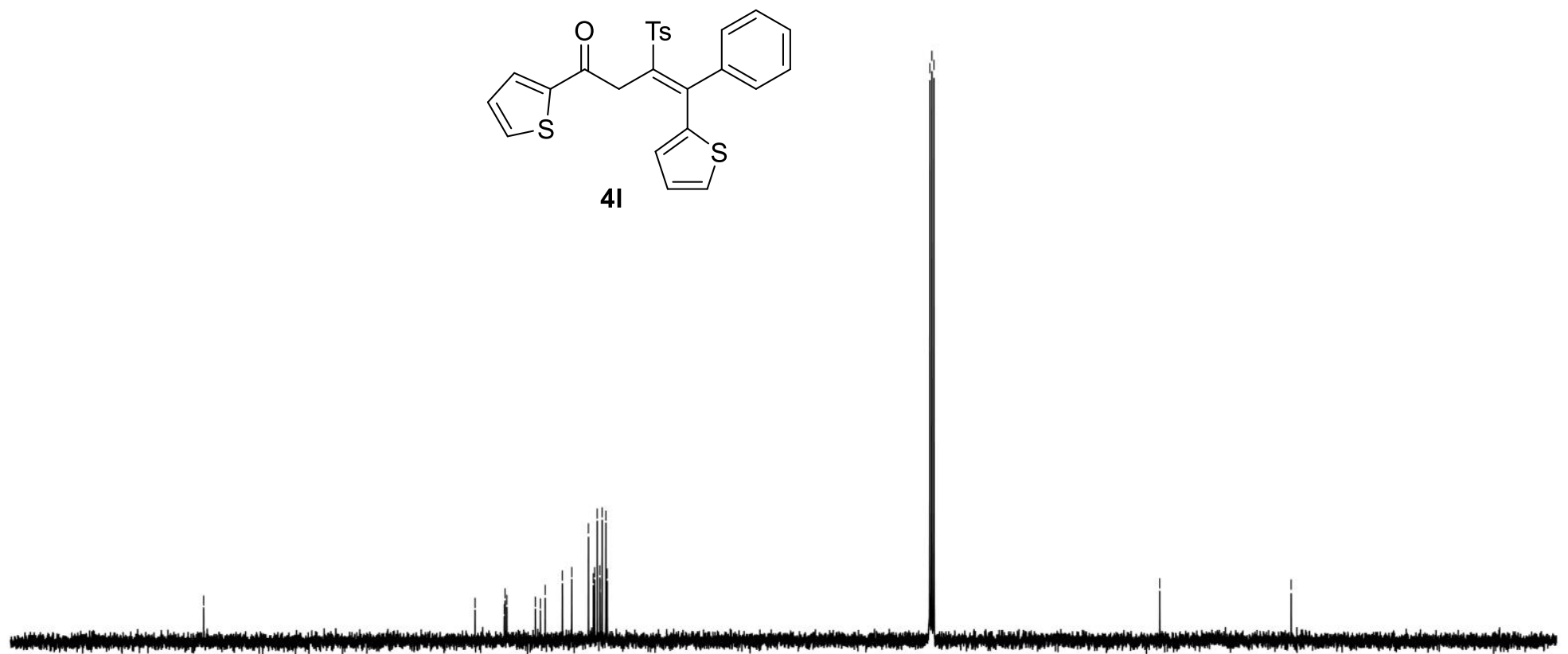

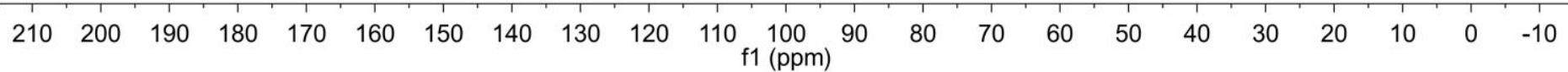




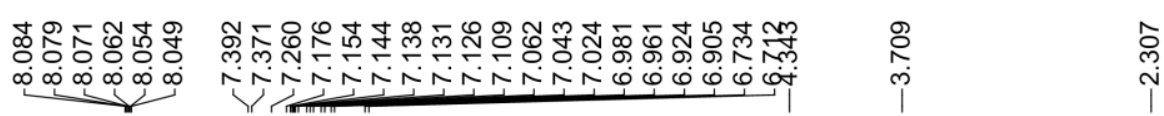
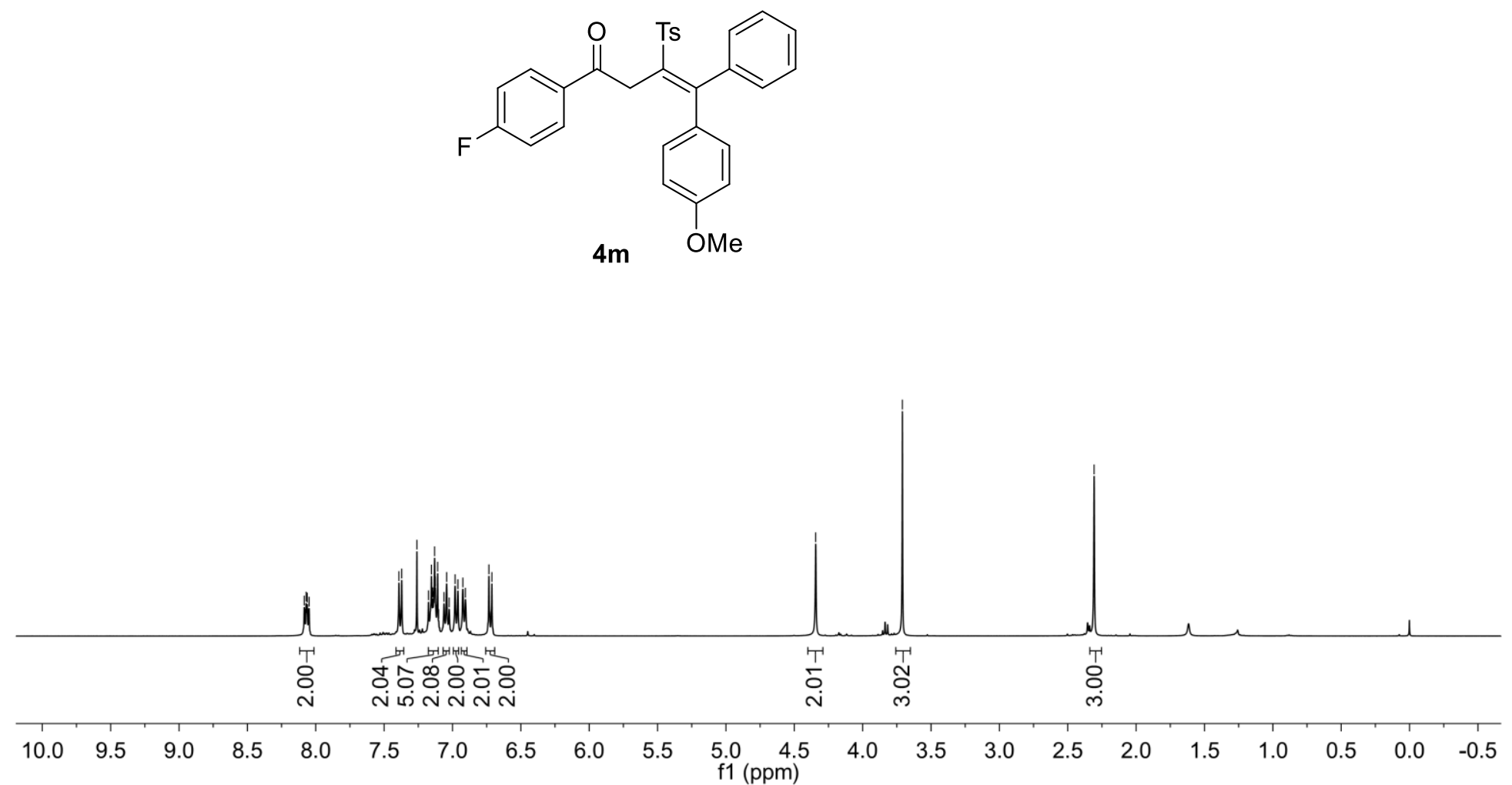


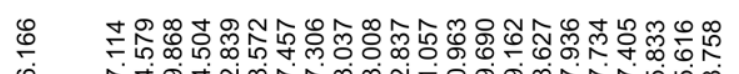

I

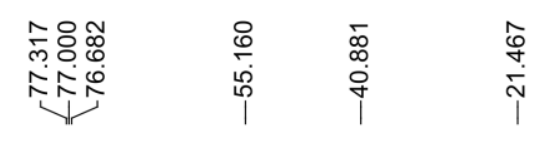

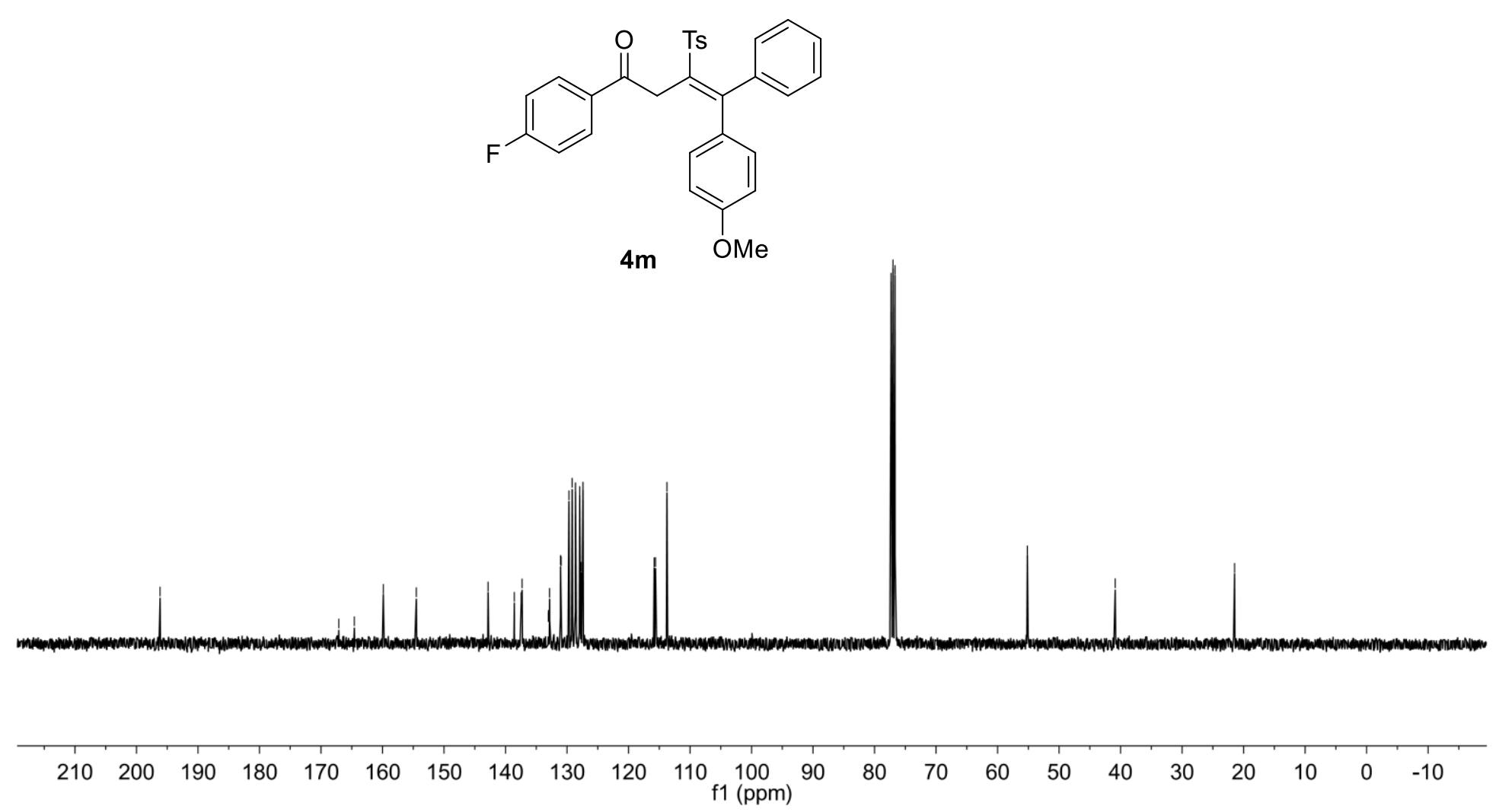



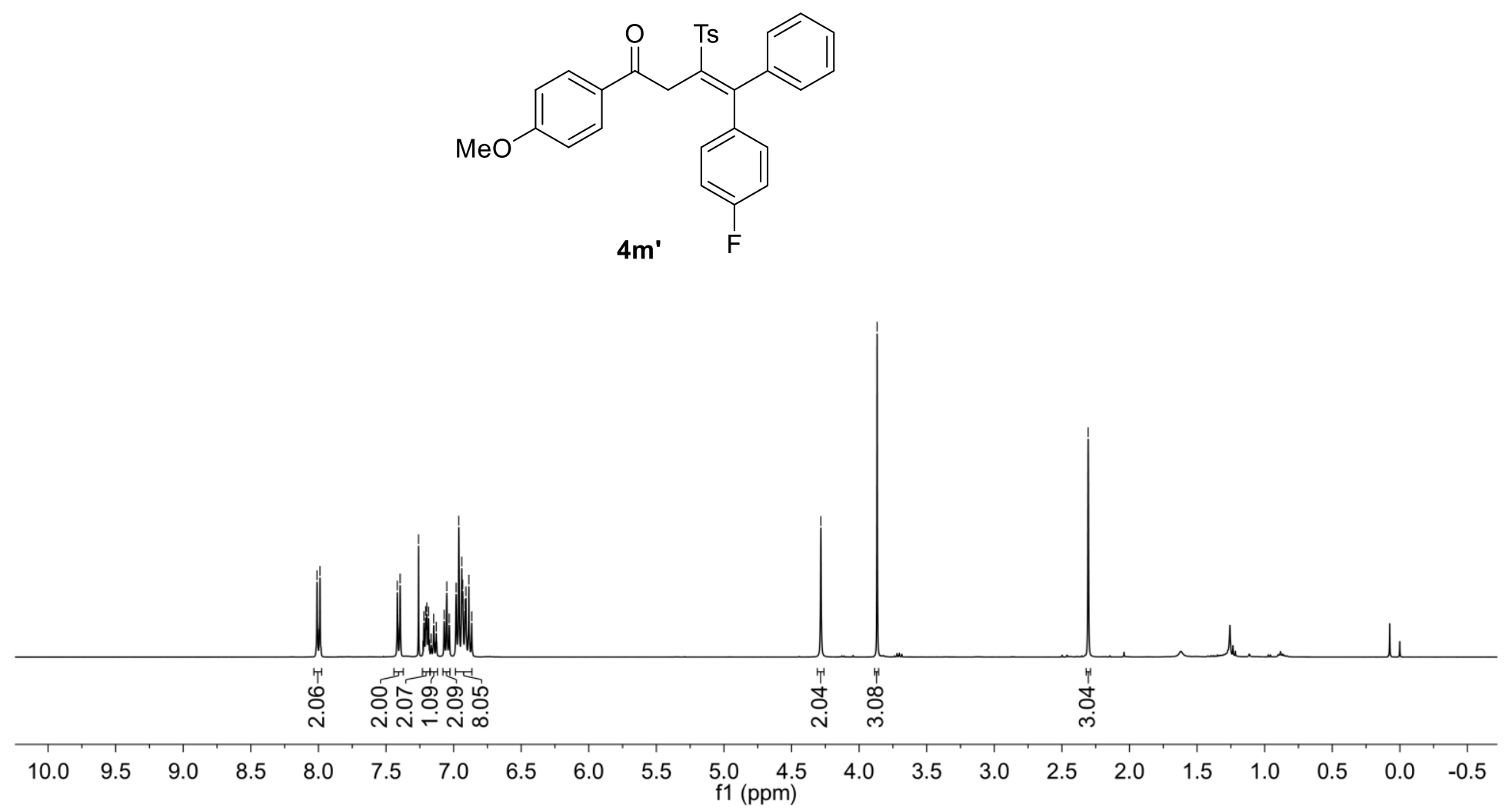

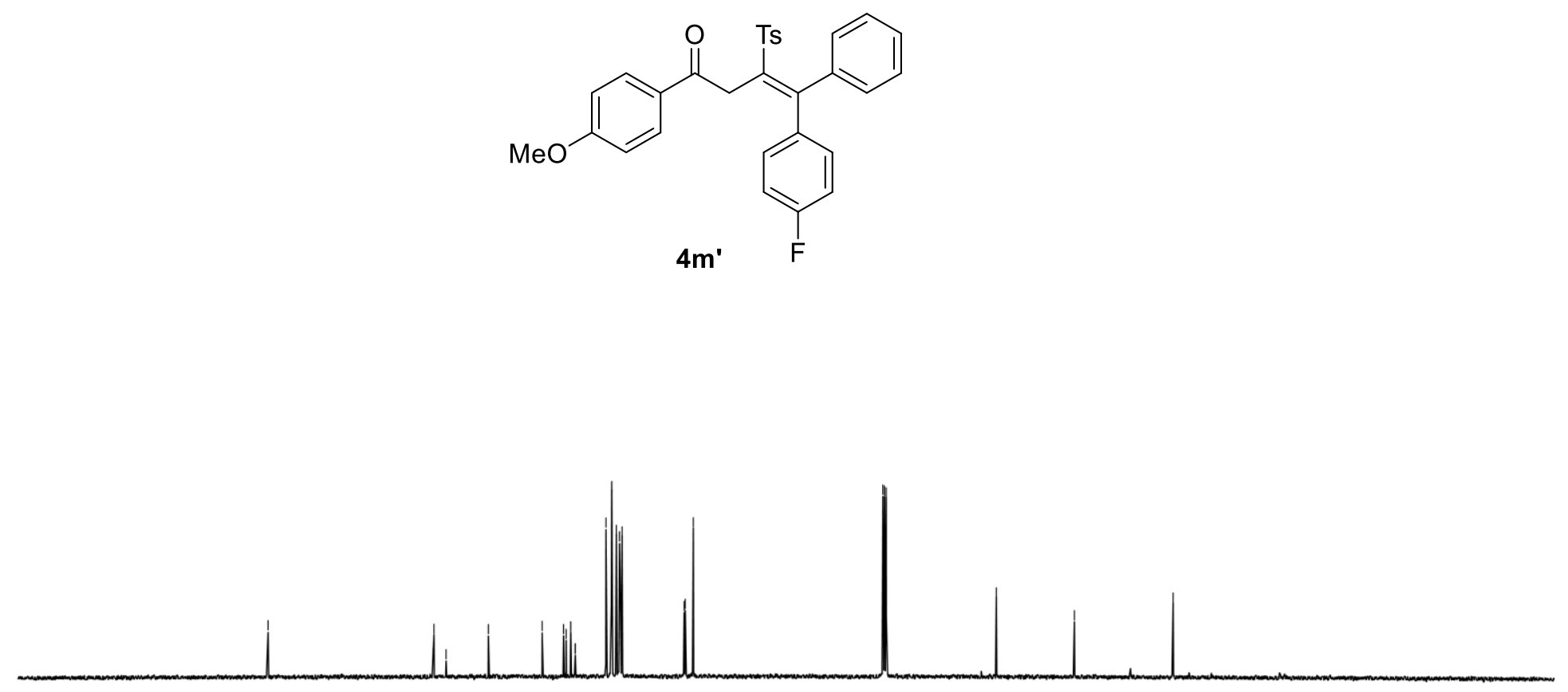

$\begin{array}{llllllllllllllllllllllllllllllll}240 & 230 & 220 & 210 & 200 & 190 & 180 & 170 & 160 & 150 & 140 & 130 & 120 & 110 & 100 & 90 & 80 & 70 & 60 & 50 & 40 & 30 & 20 & 10 & 0 & -10 & -20 & -30 & -40 & -5(\end{array}$ f1 (ppm) 


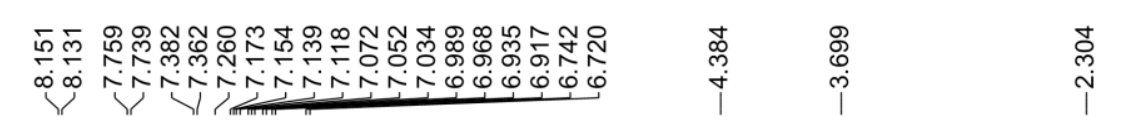
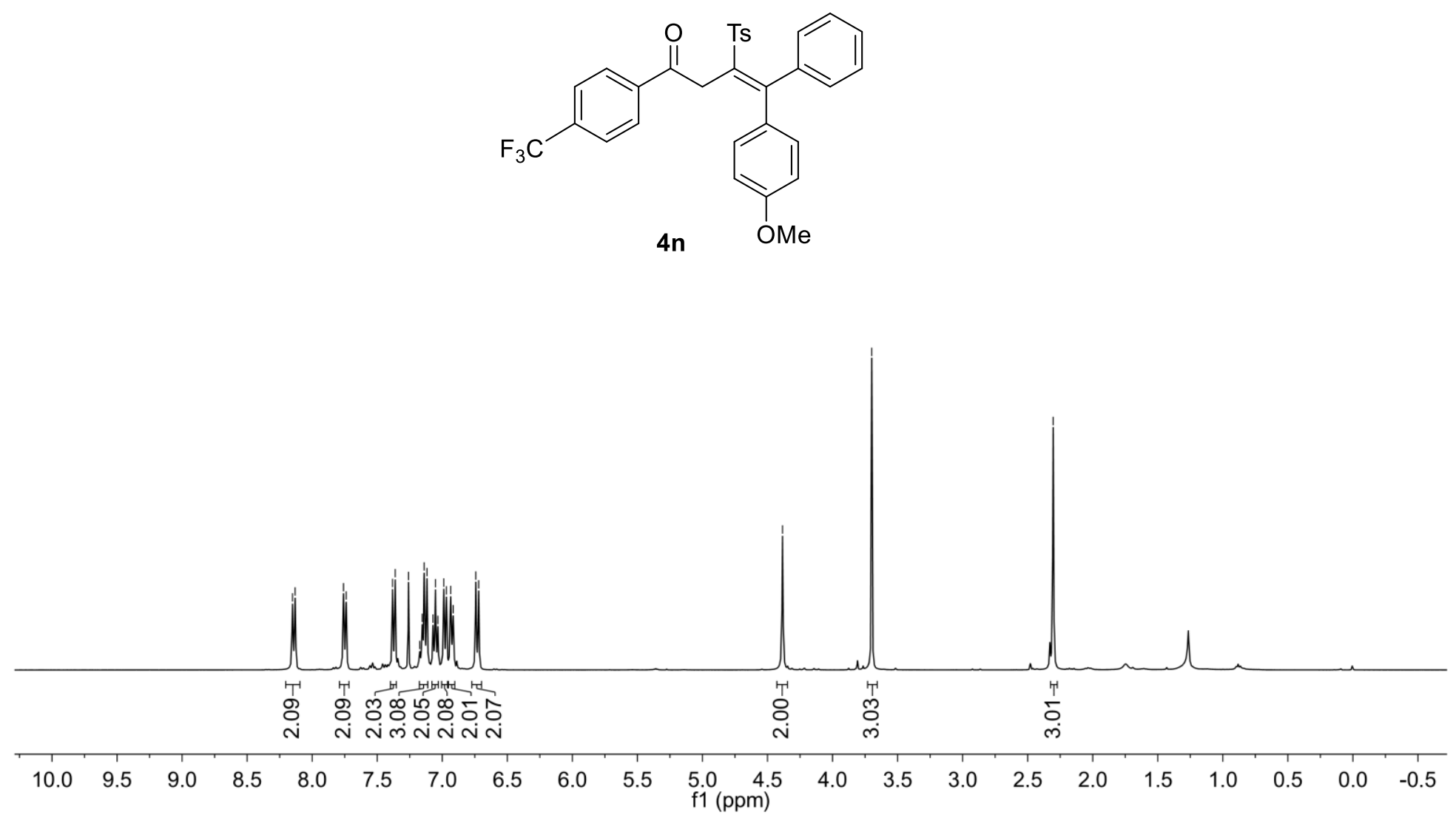


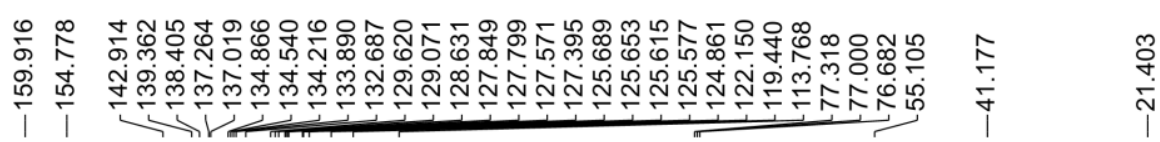
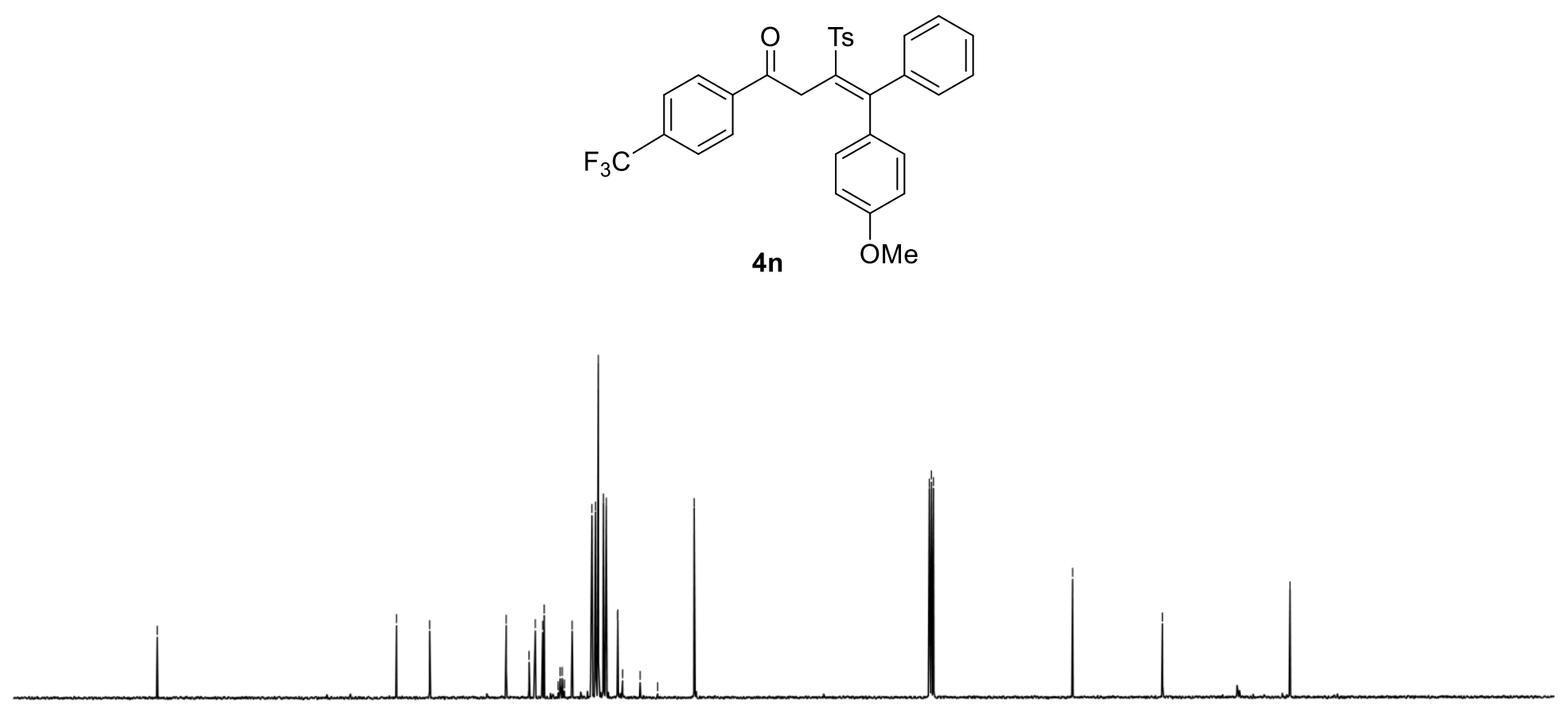

$\begin{array}{lllllllllllllllllllllll}210 & 200 & 190 & 180 & 170 & 160 & 150 & 140 & 130 & 120 & 110 & \begin{array}{c}100 \\ \mathrm{f} 1(\mathrm{ppm})\end{array} & 90 & 80 & 70 & 60 & 50 & 40 & 30 & 20 & 10 & 0 & -10\end{array}$ 

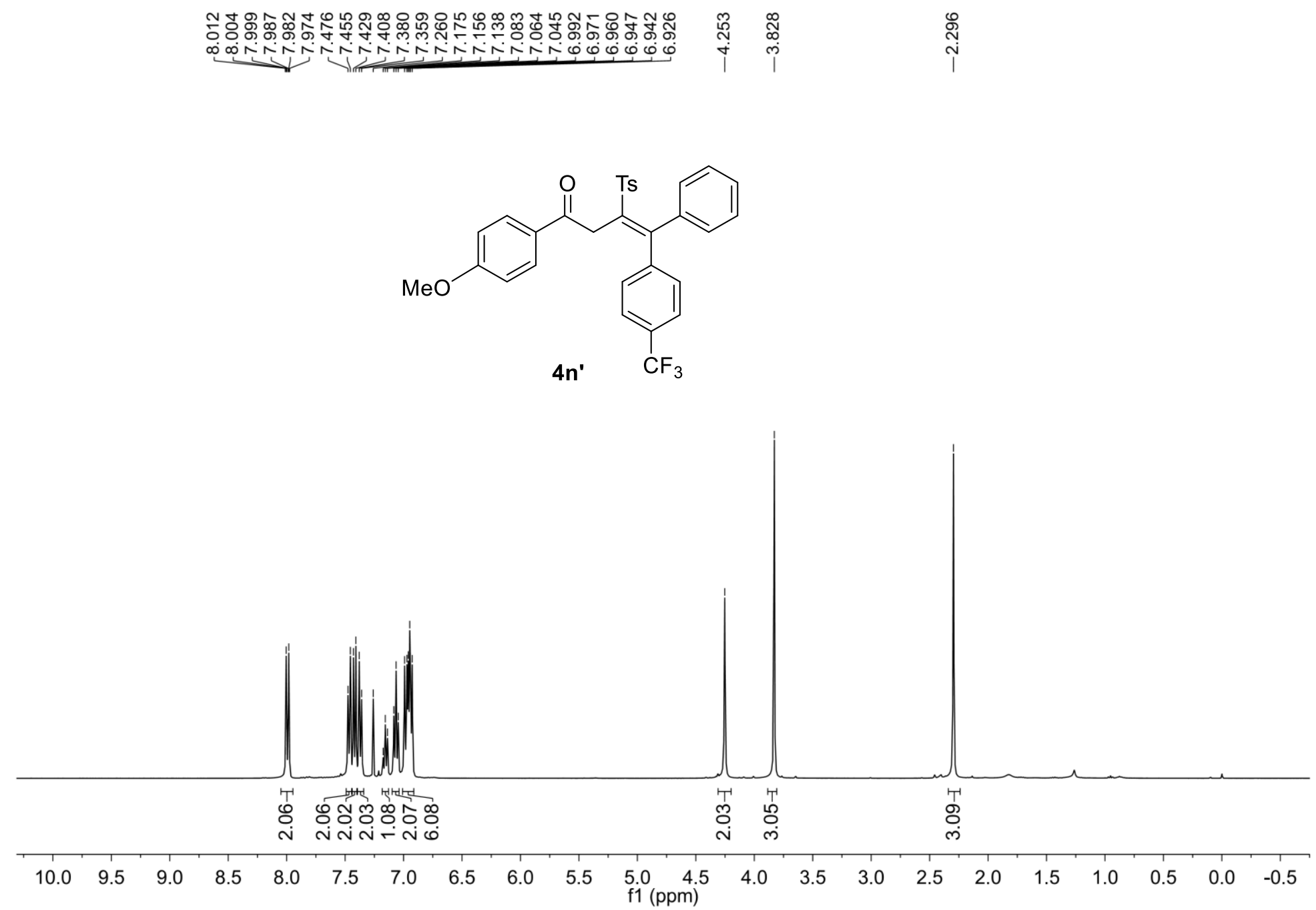


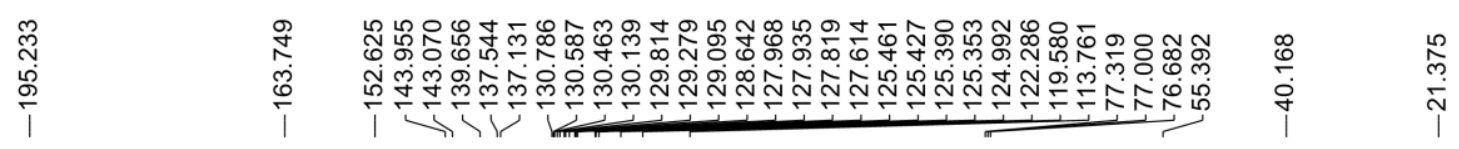
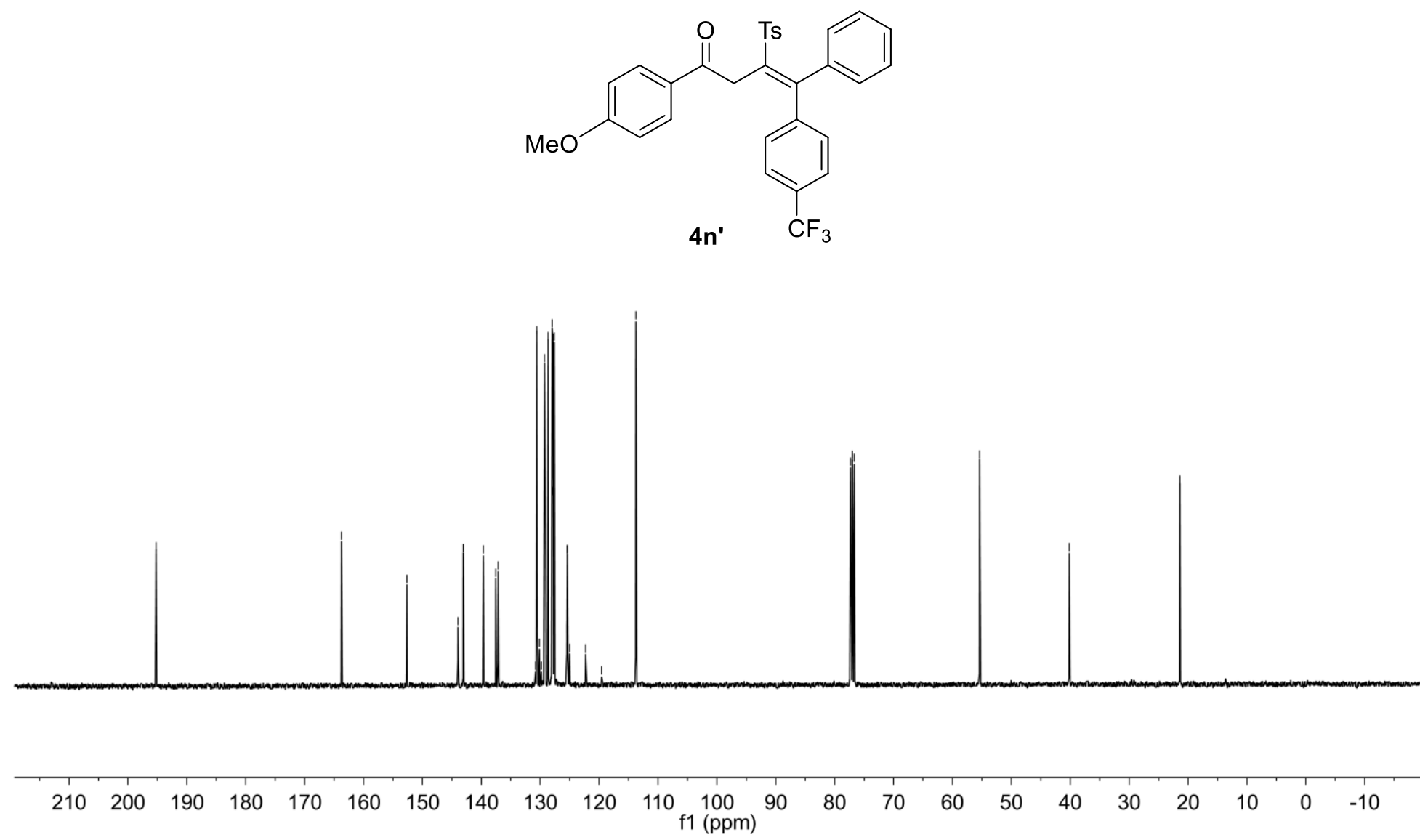


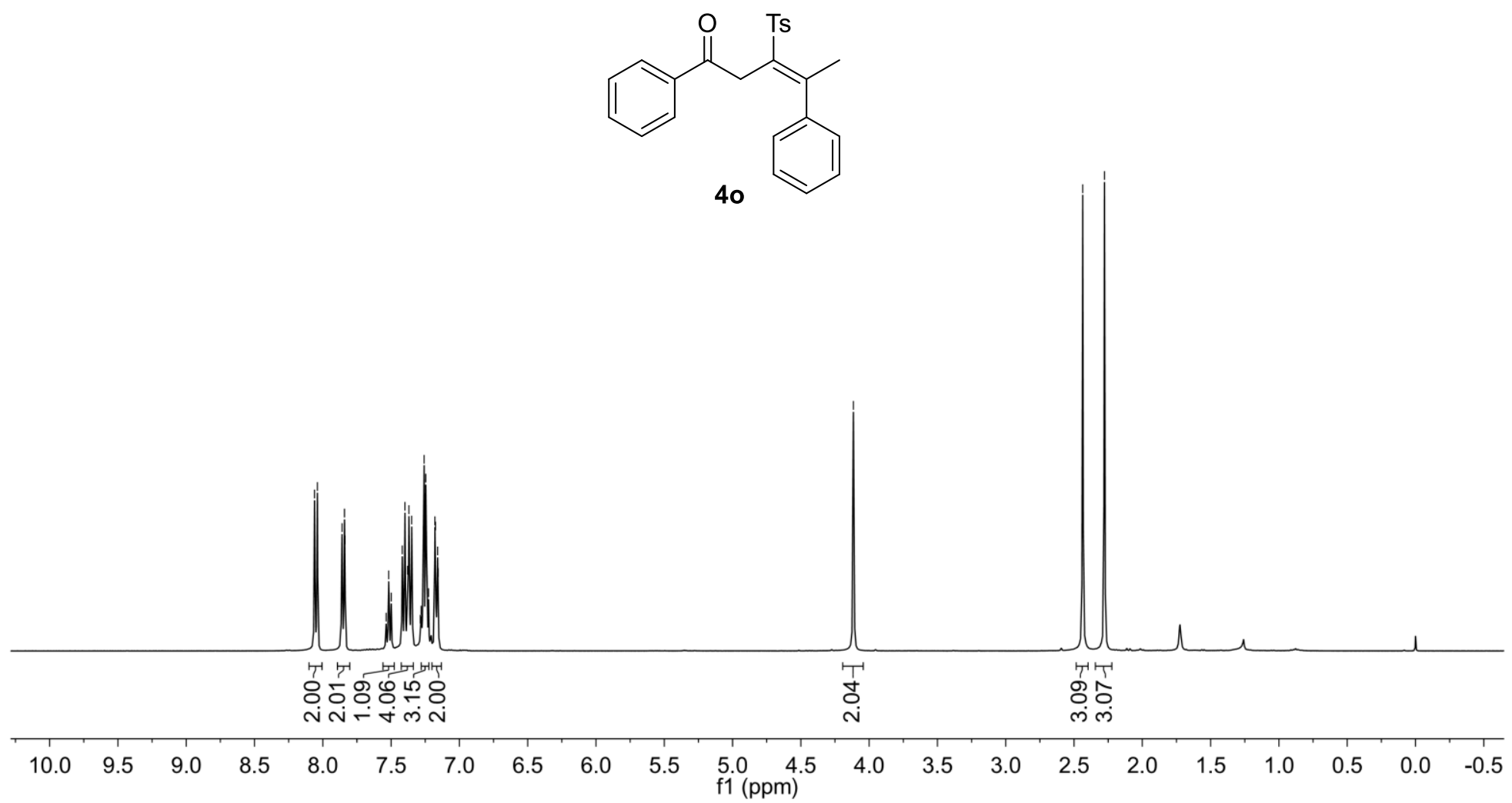



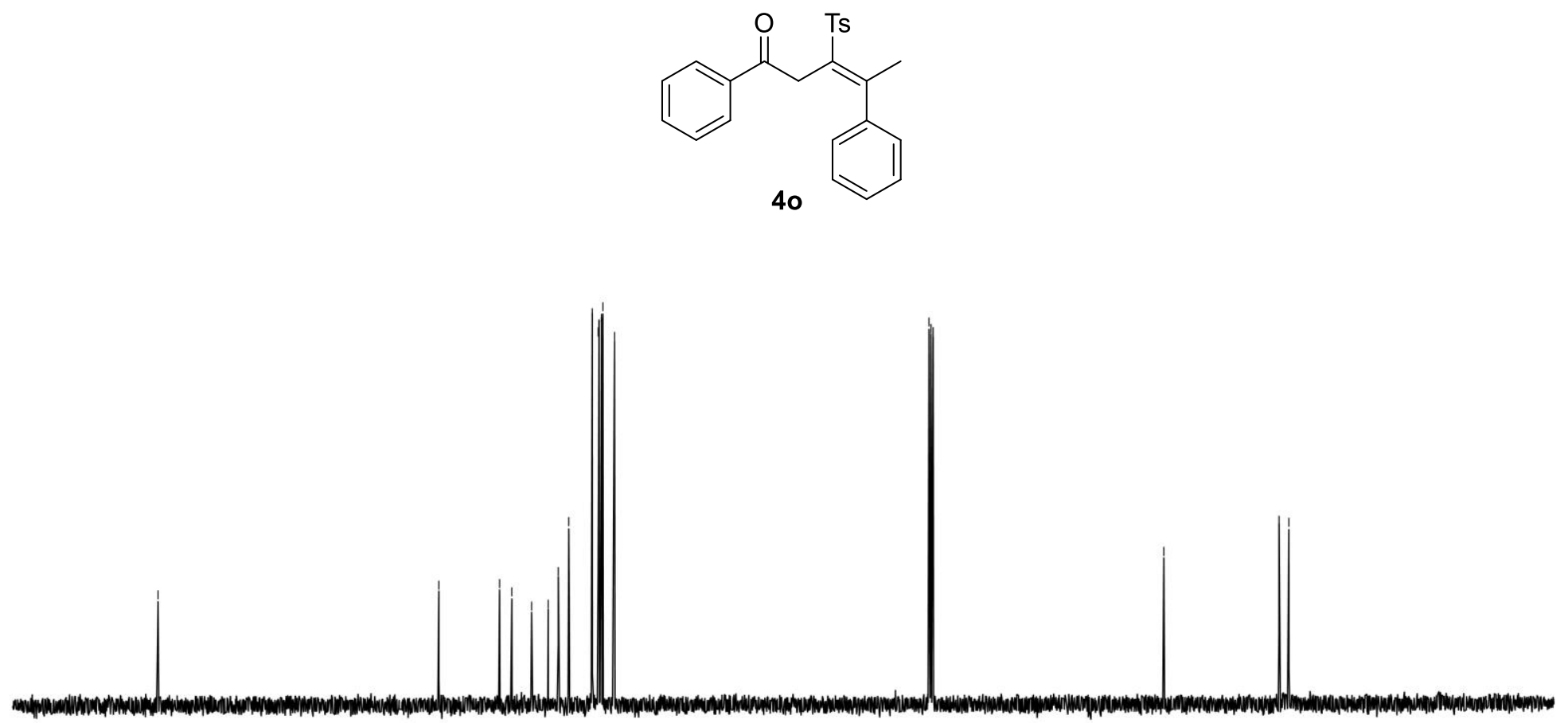

$\begin{array}{llllllllllllllllllllll}210 & 200 & 190 & 180 & 170 & 160 & 150 & 140 & 130 & 120 & 110 \begin{array}{c}100 \\ \mathrm{f} 1(\mathrm{ppm})\end{array} & 90 & 80 & 70 & 60 & 50 & 40 & 30 & 20 & 10 & 0 & -10\end{array}$ 


\section{罂英}

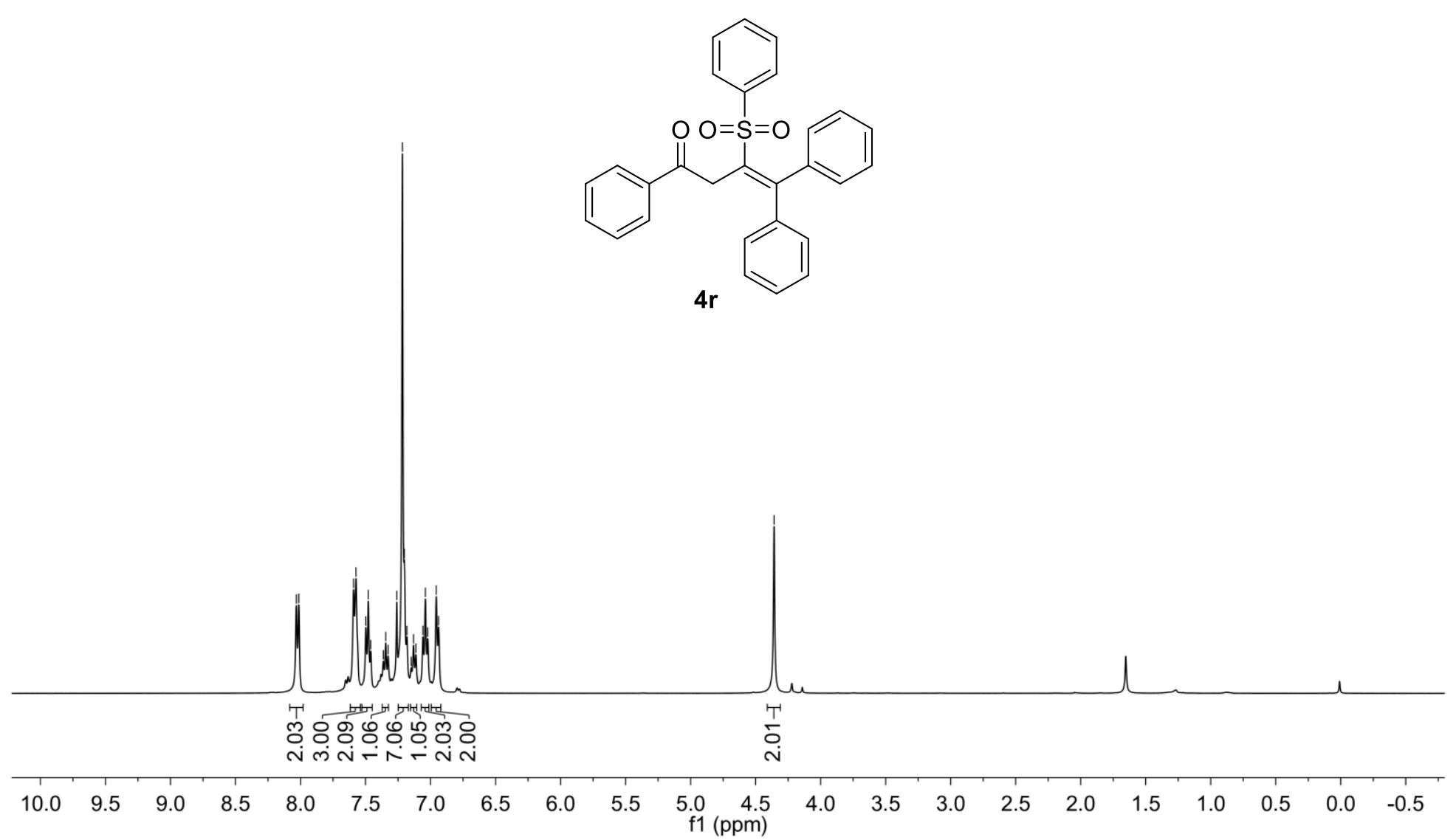



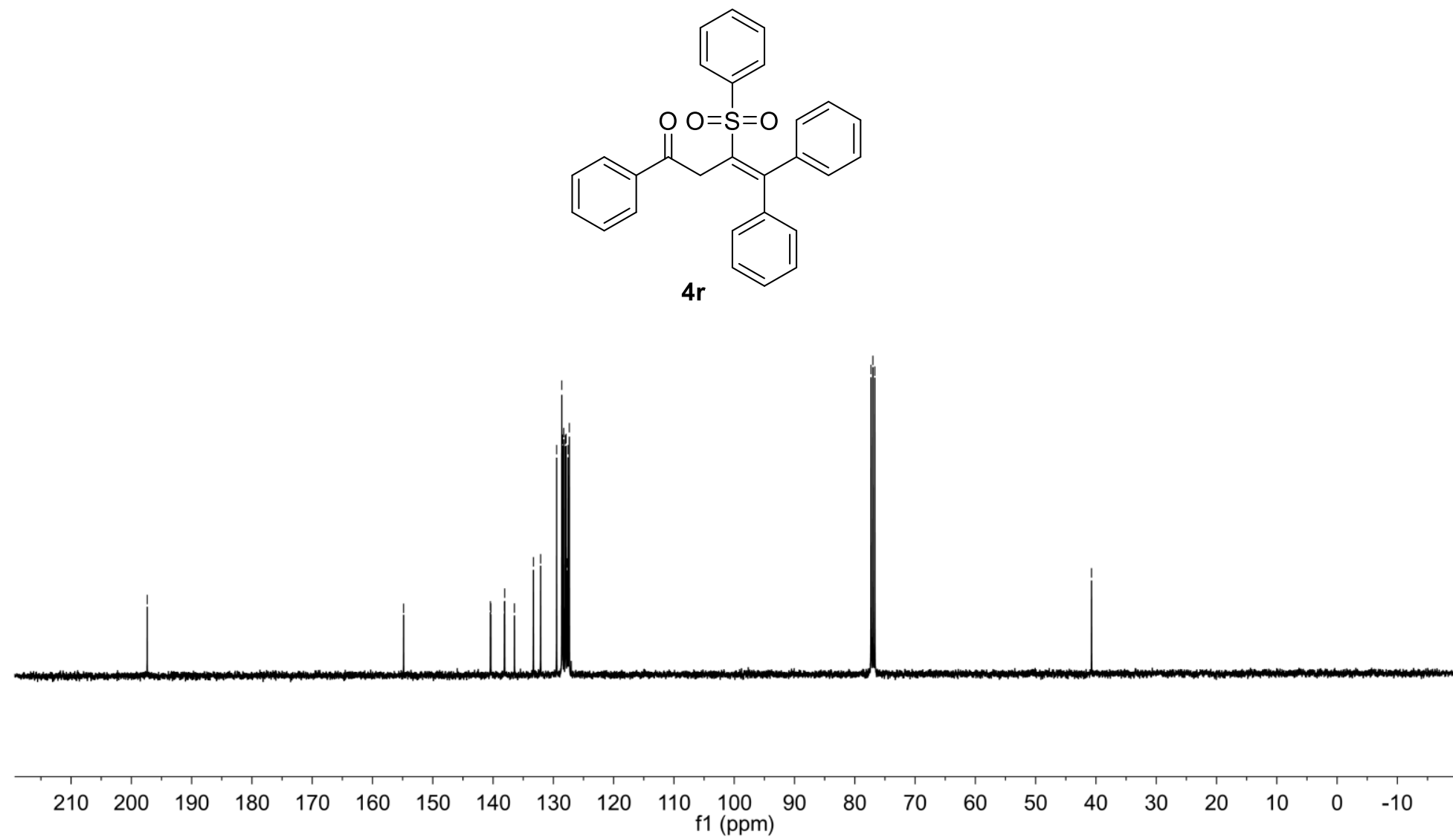

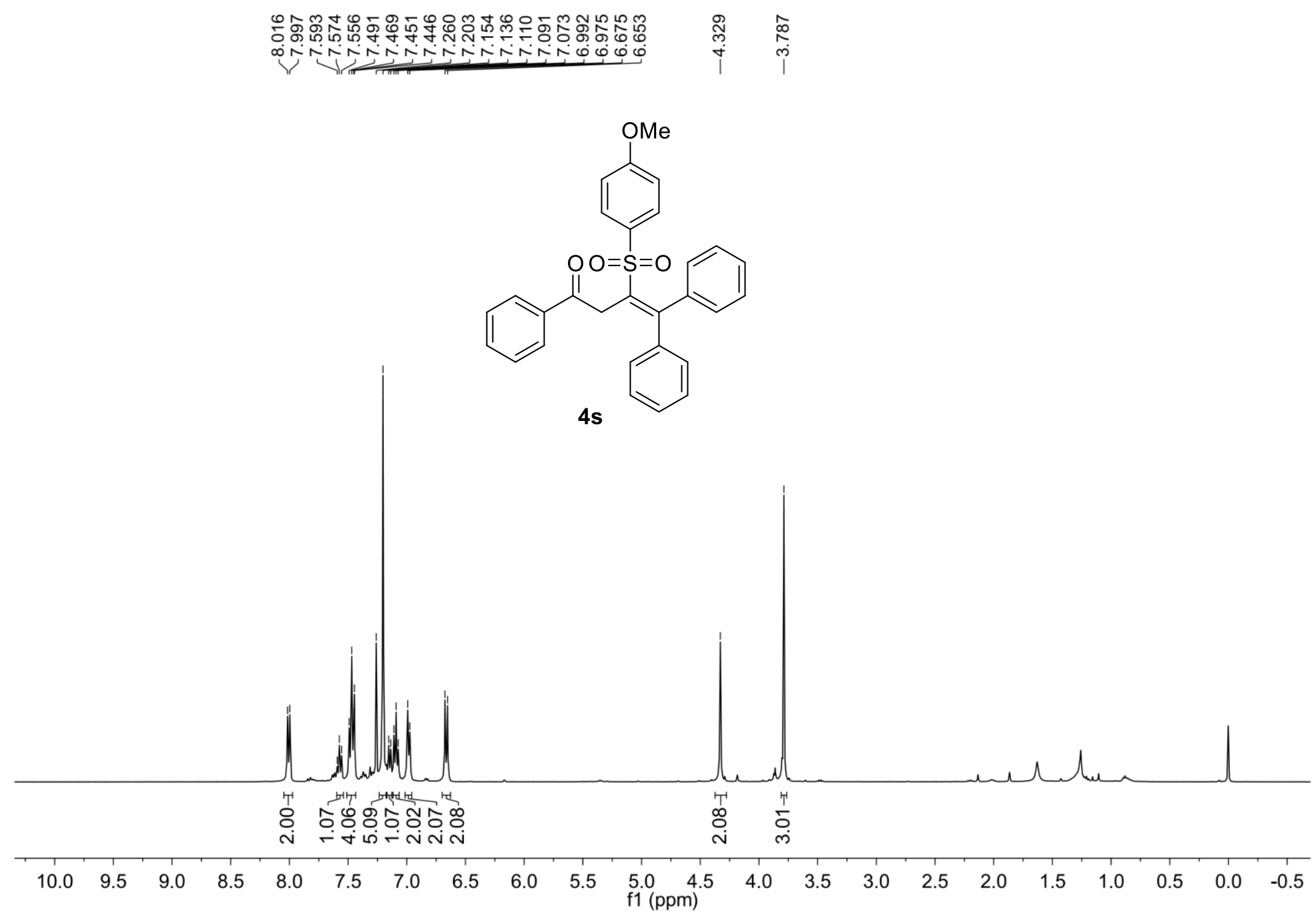


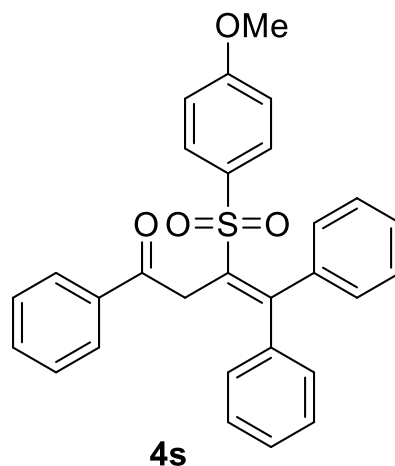



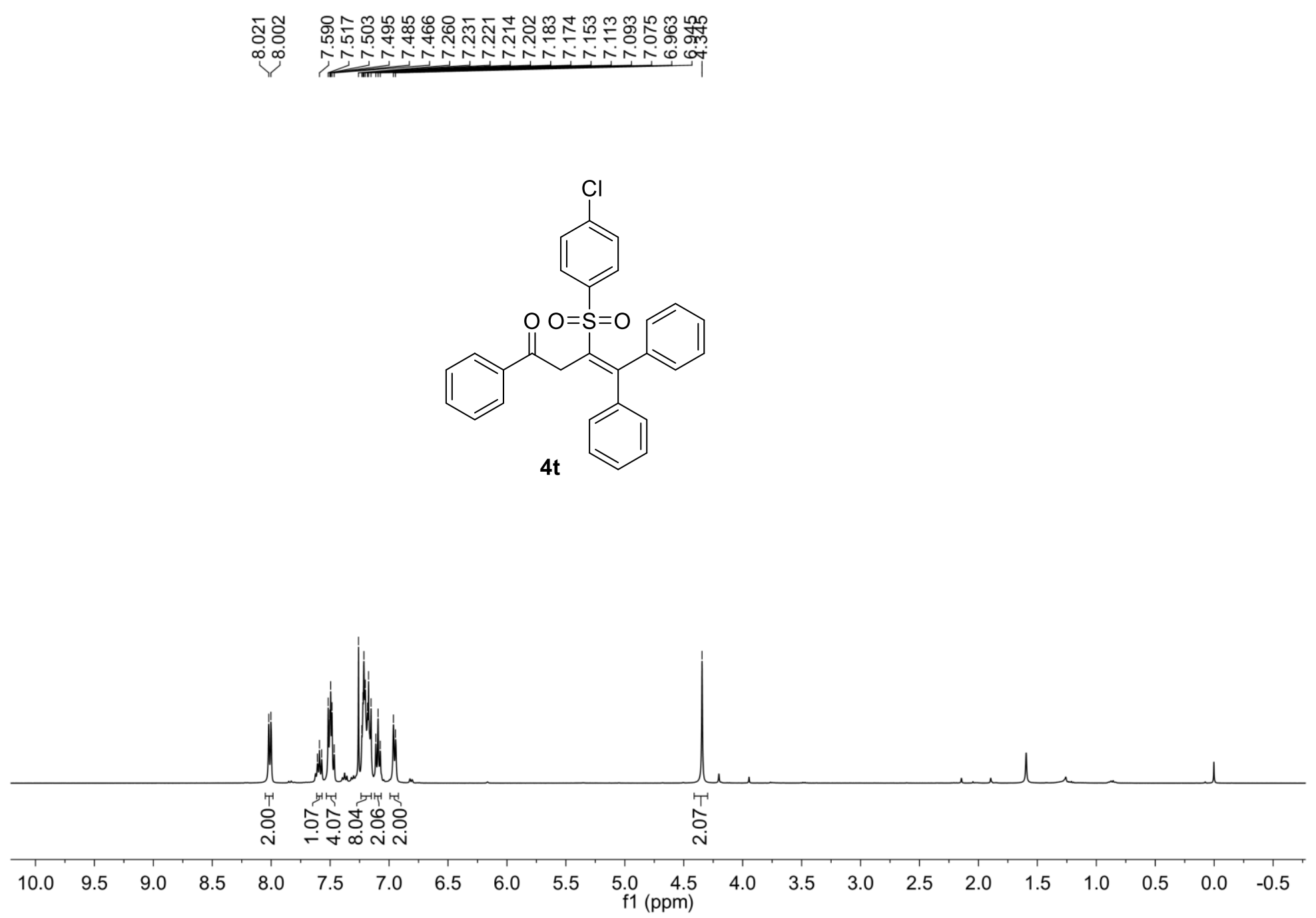

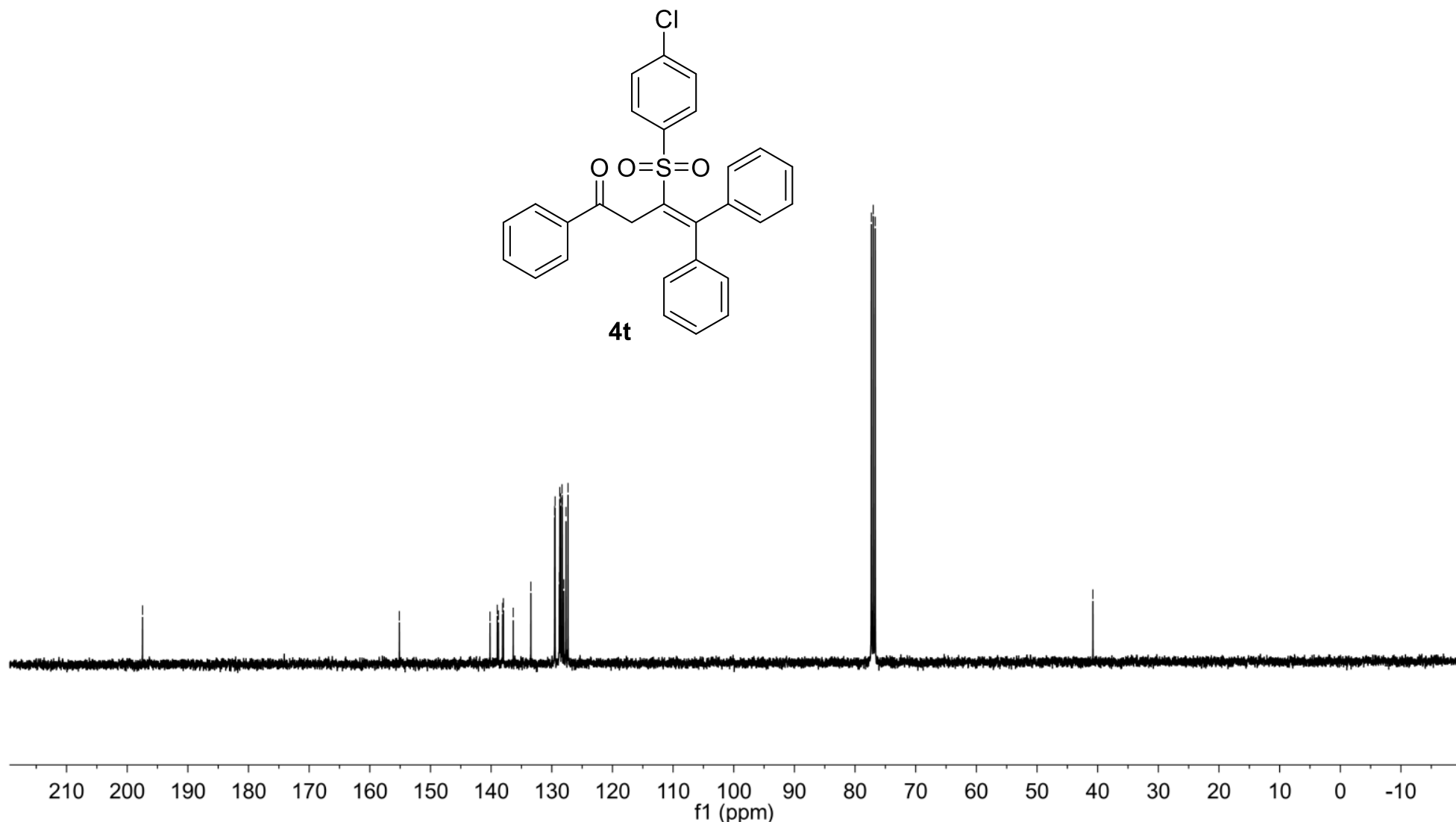


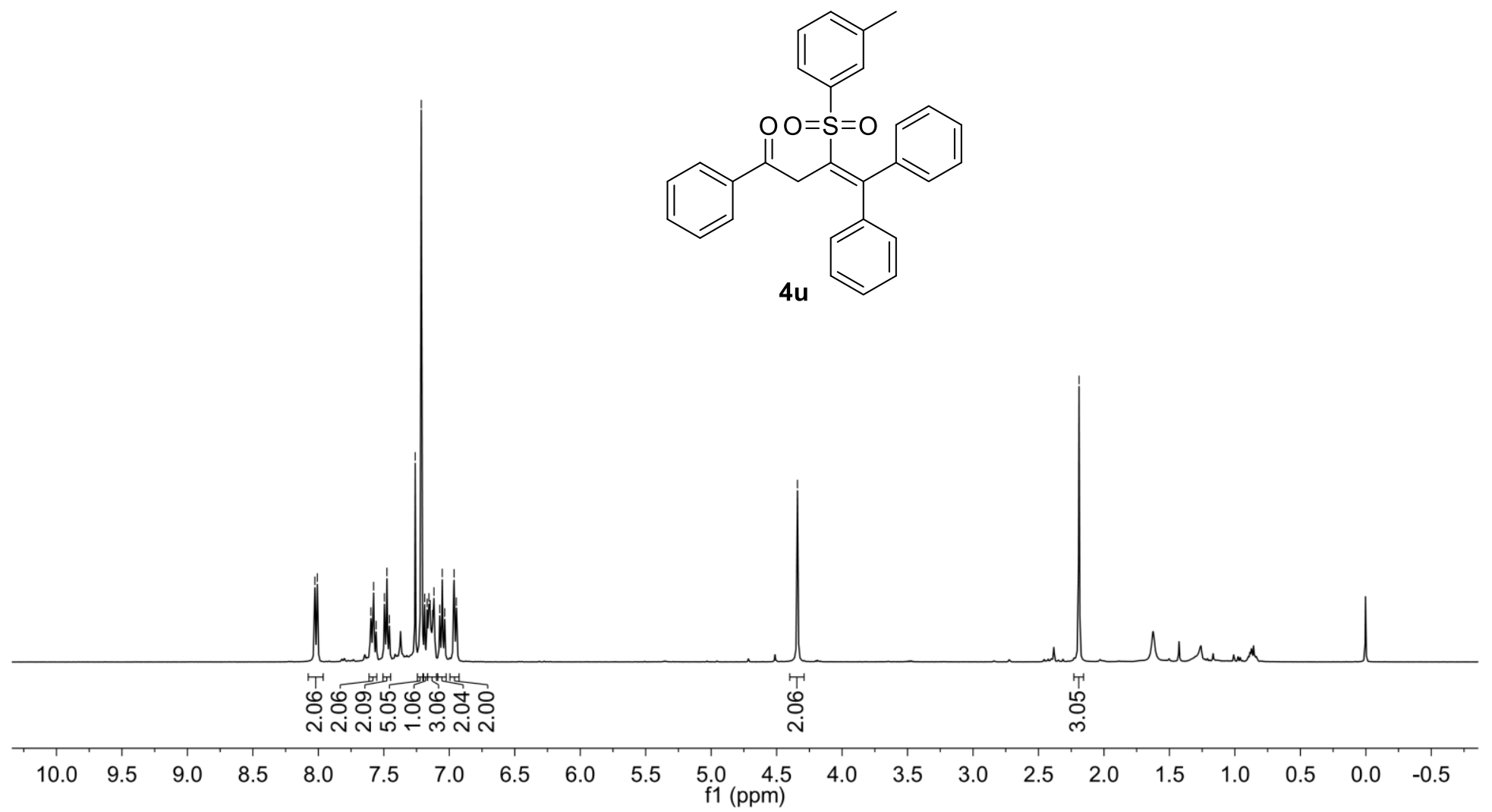




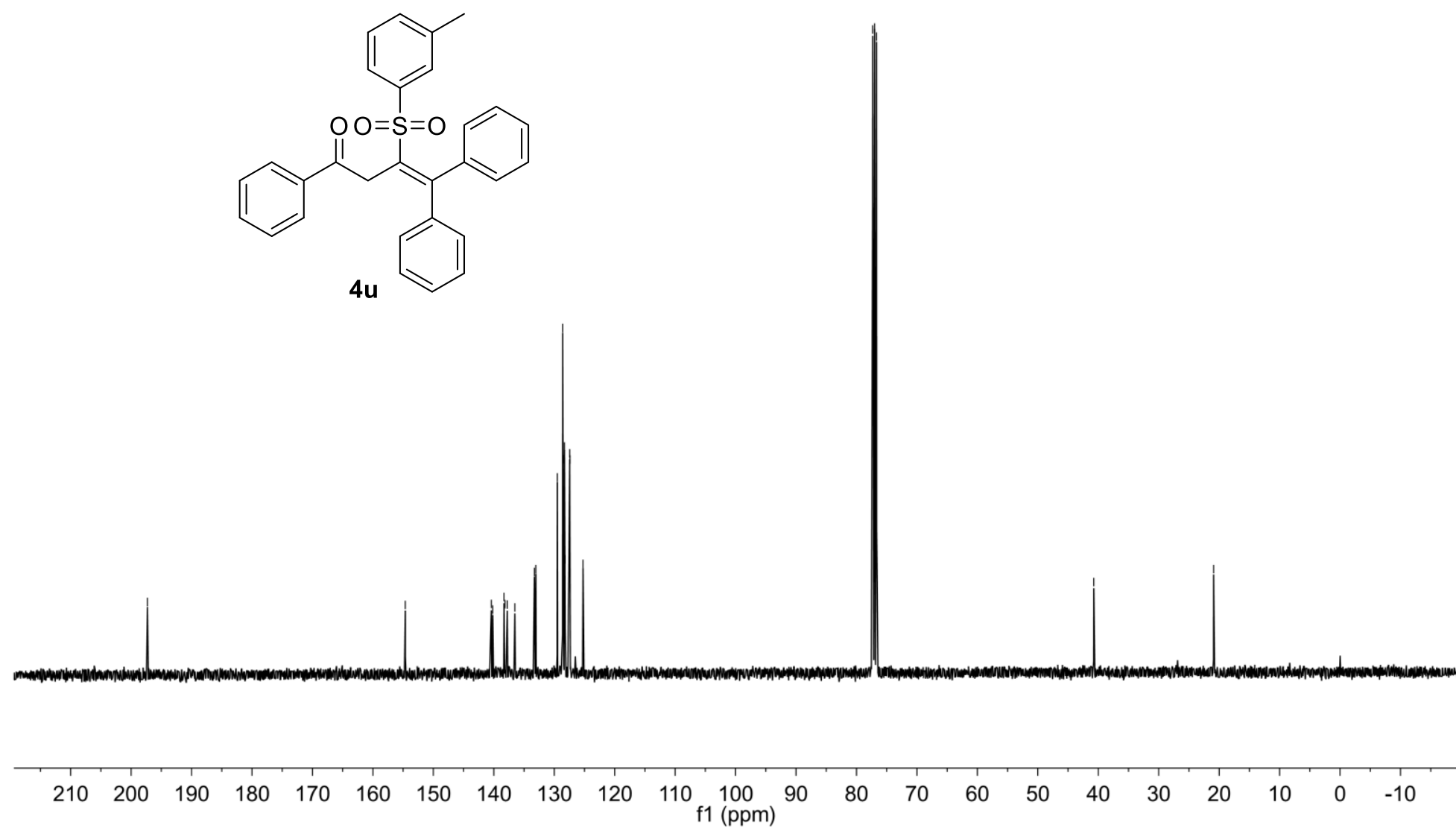




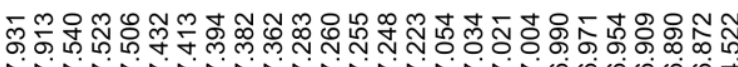

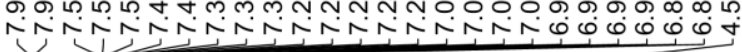
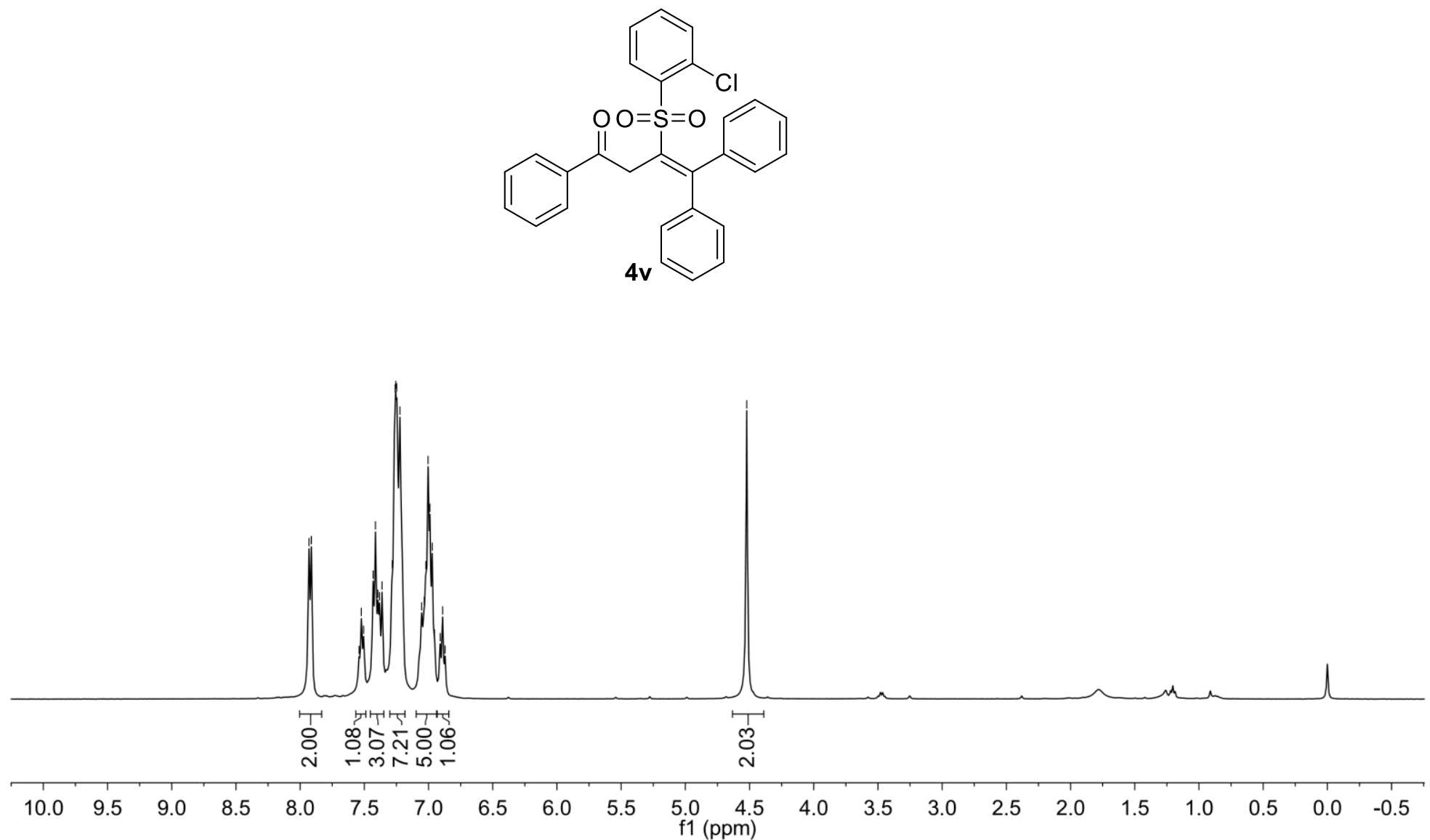


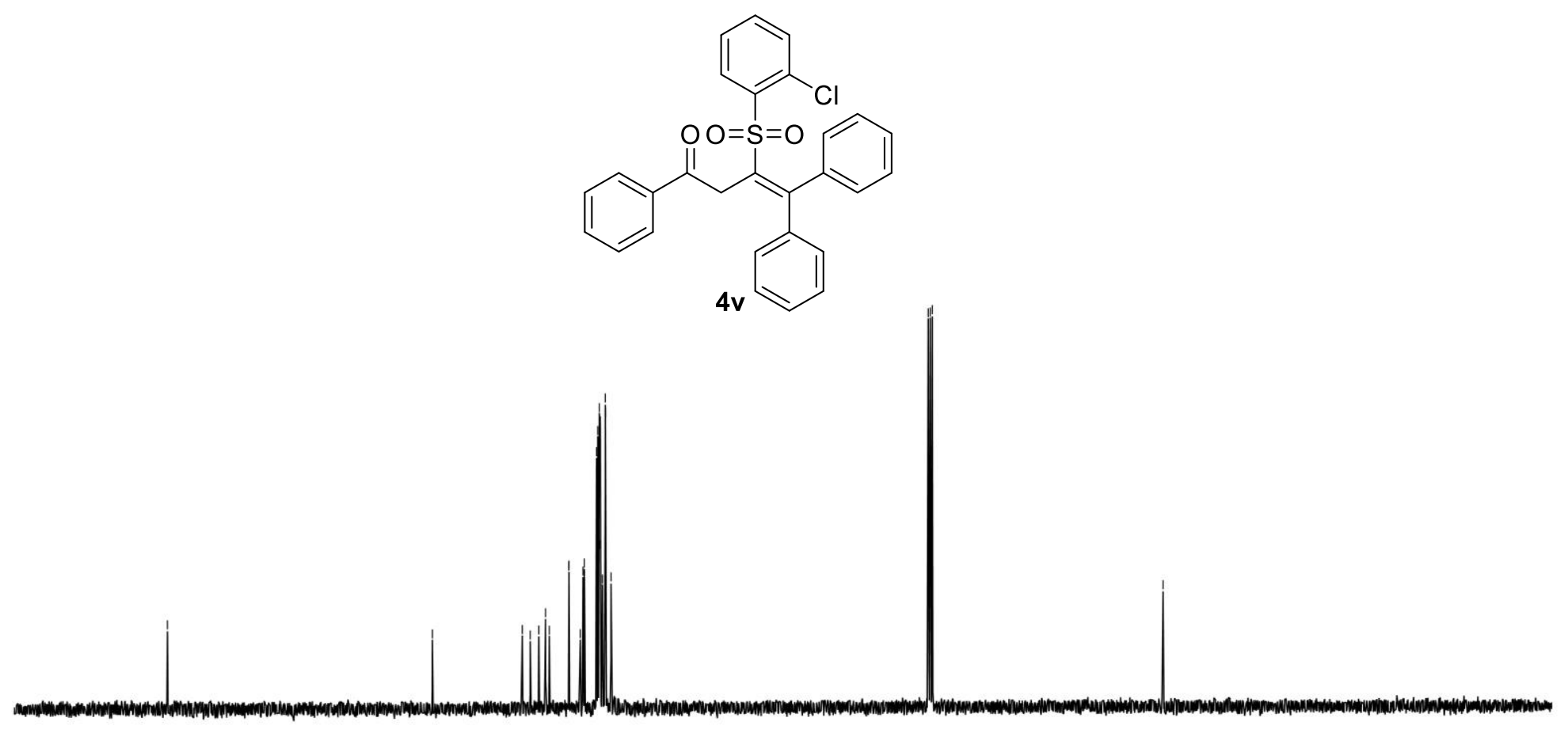

$\begin{array}{llllllllllll}210 & 200 & 190 & 180 & 170 & 160 & 150 & 140 & 130 & 120 & 110 & 100\end{array}$ f1 $(\mathrm{ppm})$ $\begin{array}{llllllllll}80 & 70 & 60 & 50 & 40 & 30 & 20 & 10 & 0 & -10\end{array}$ 


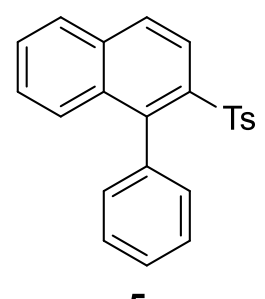

5a

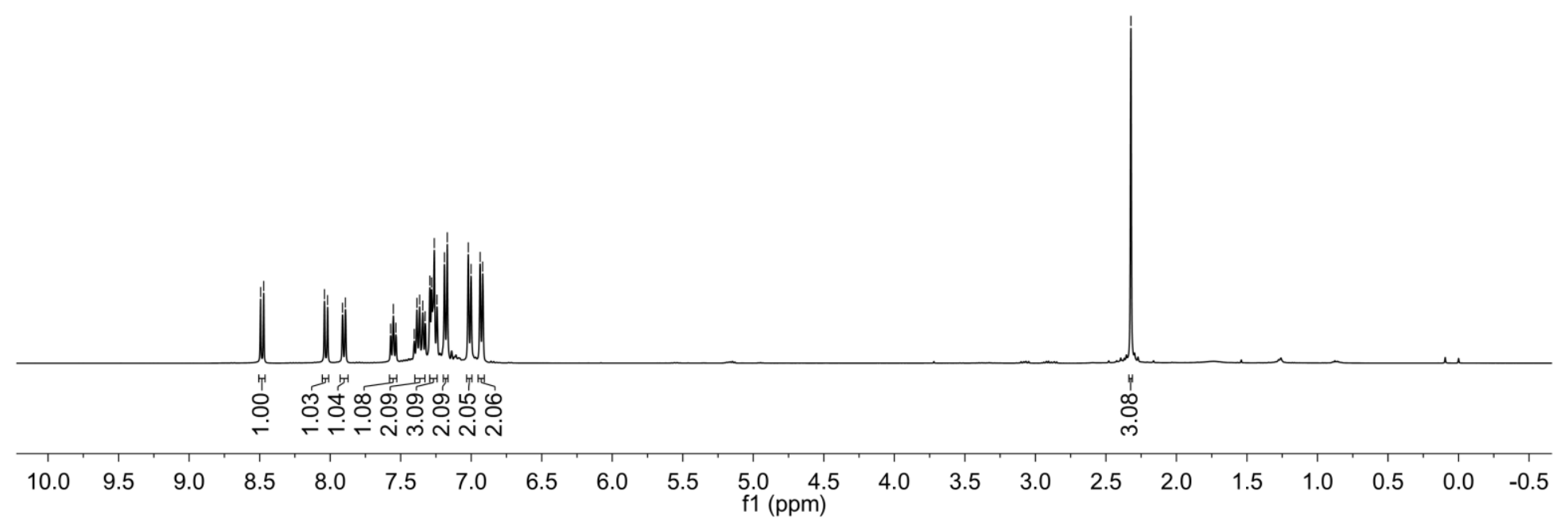




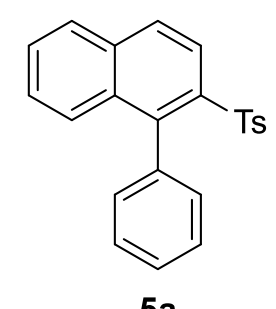

$5 a$

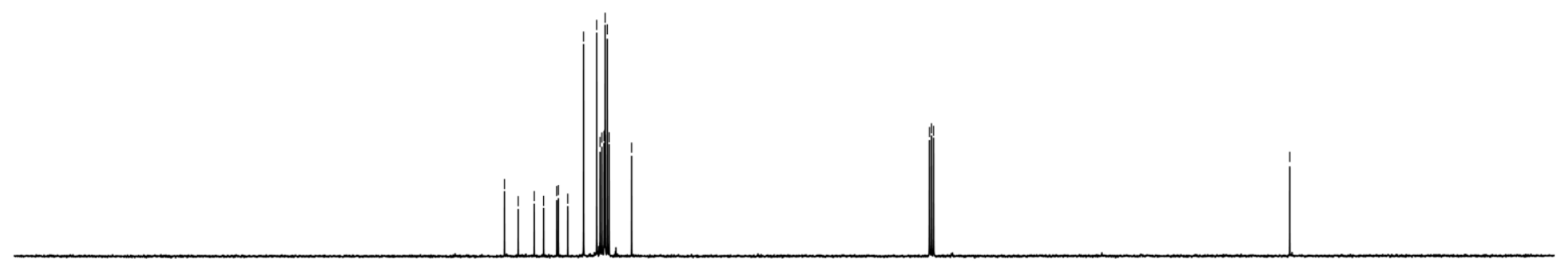

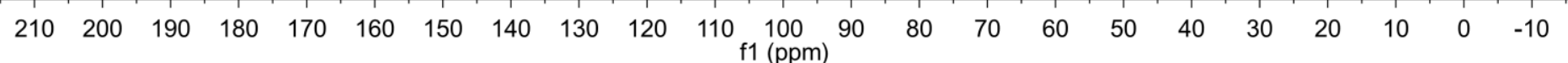




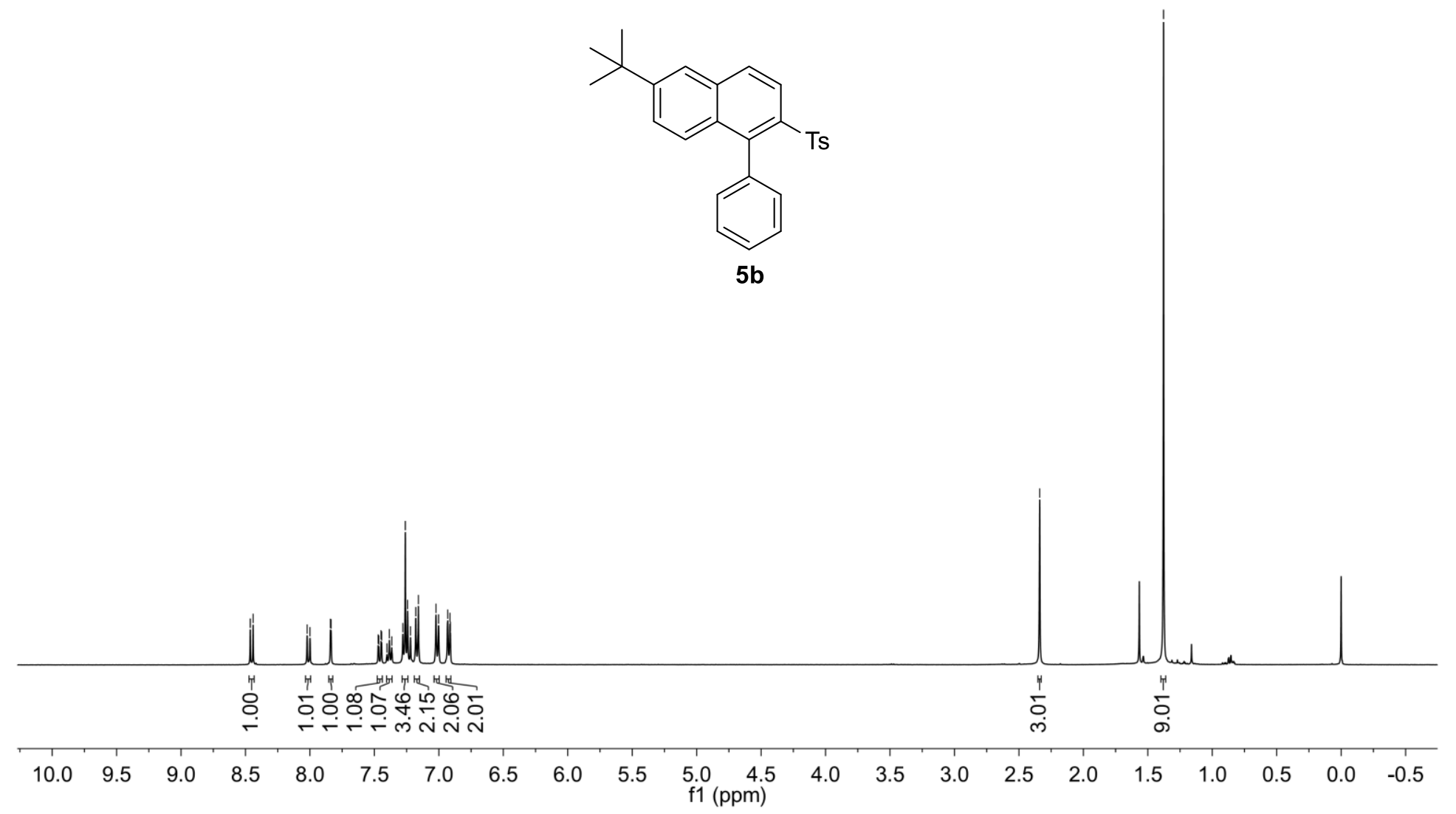



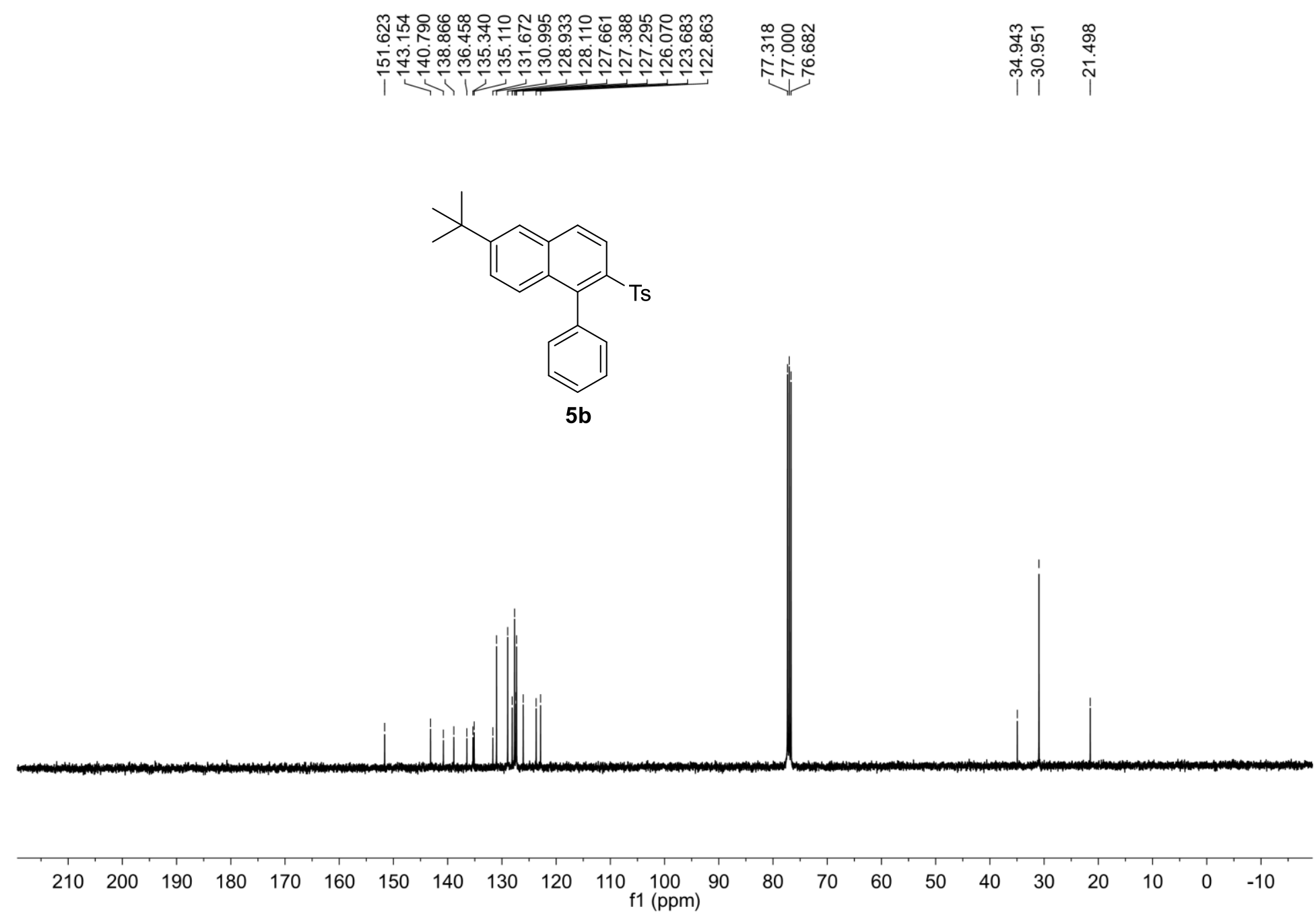

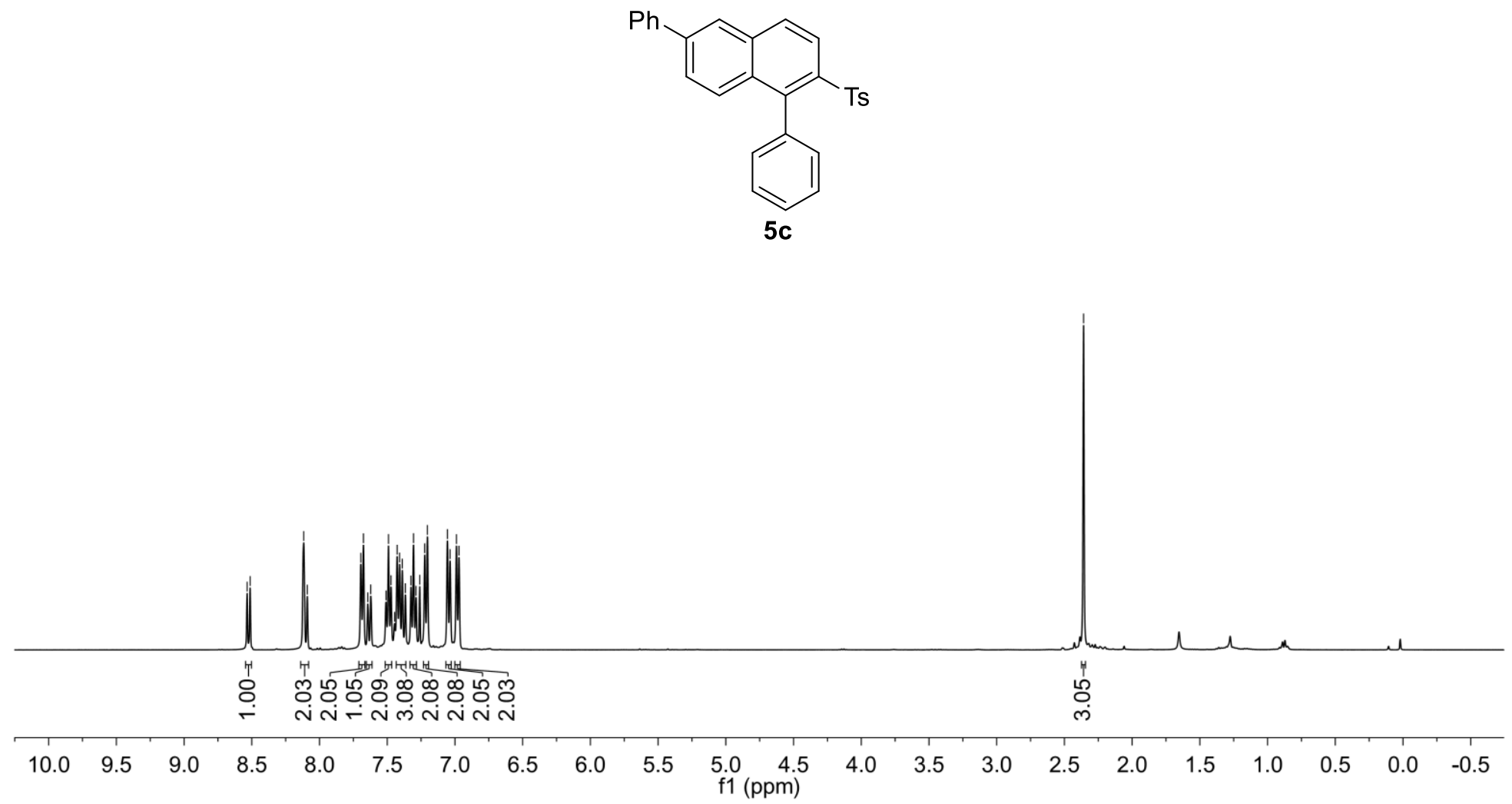

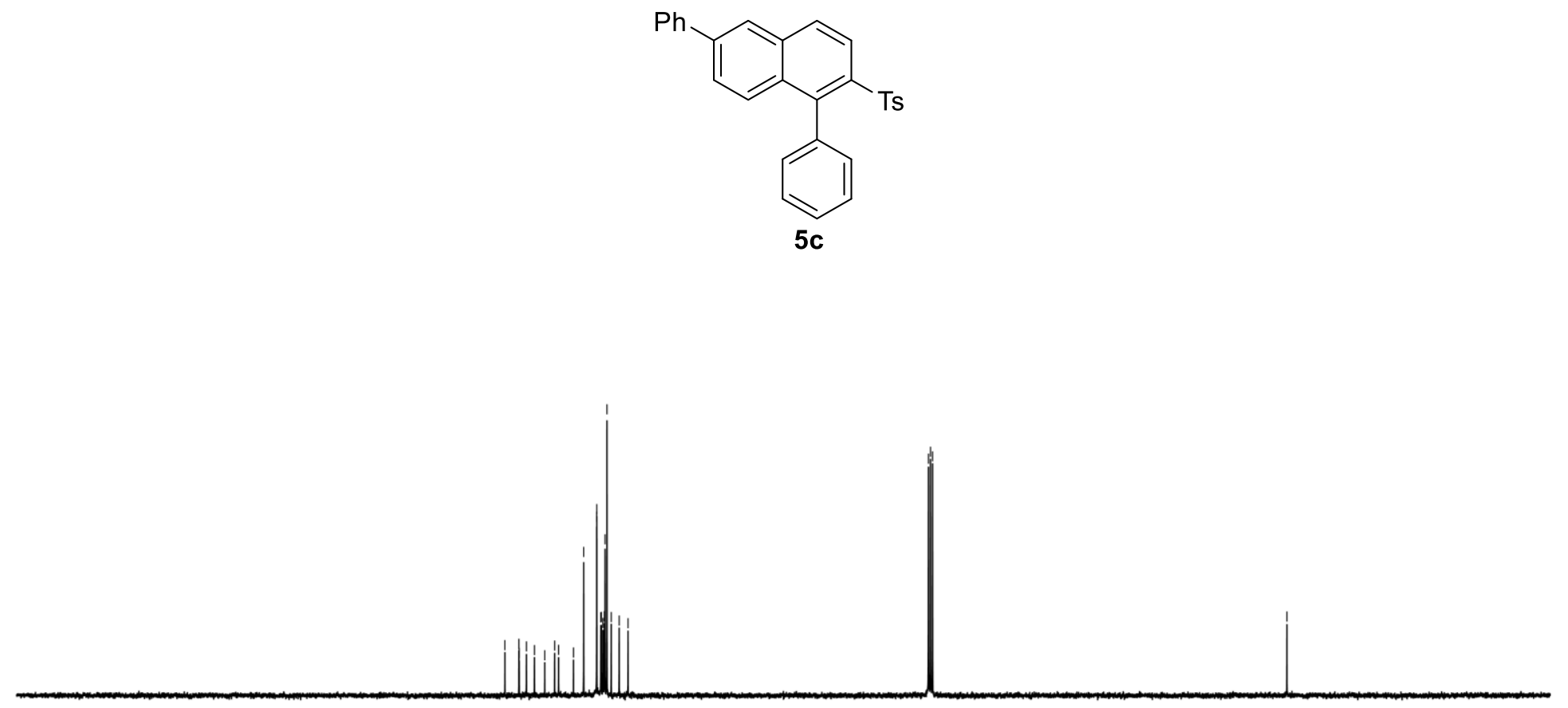

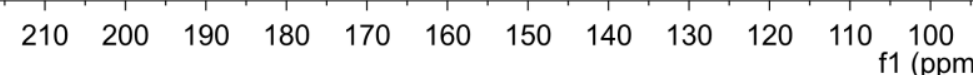



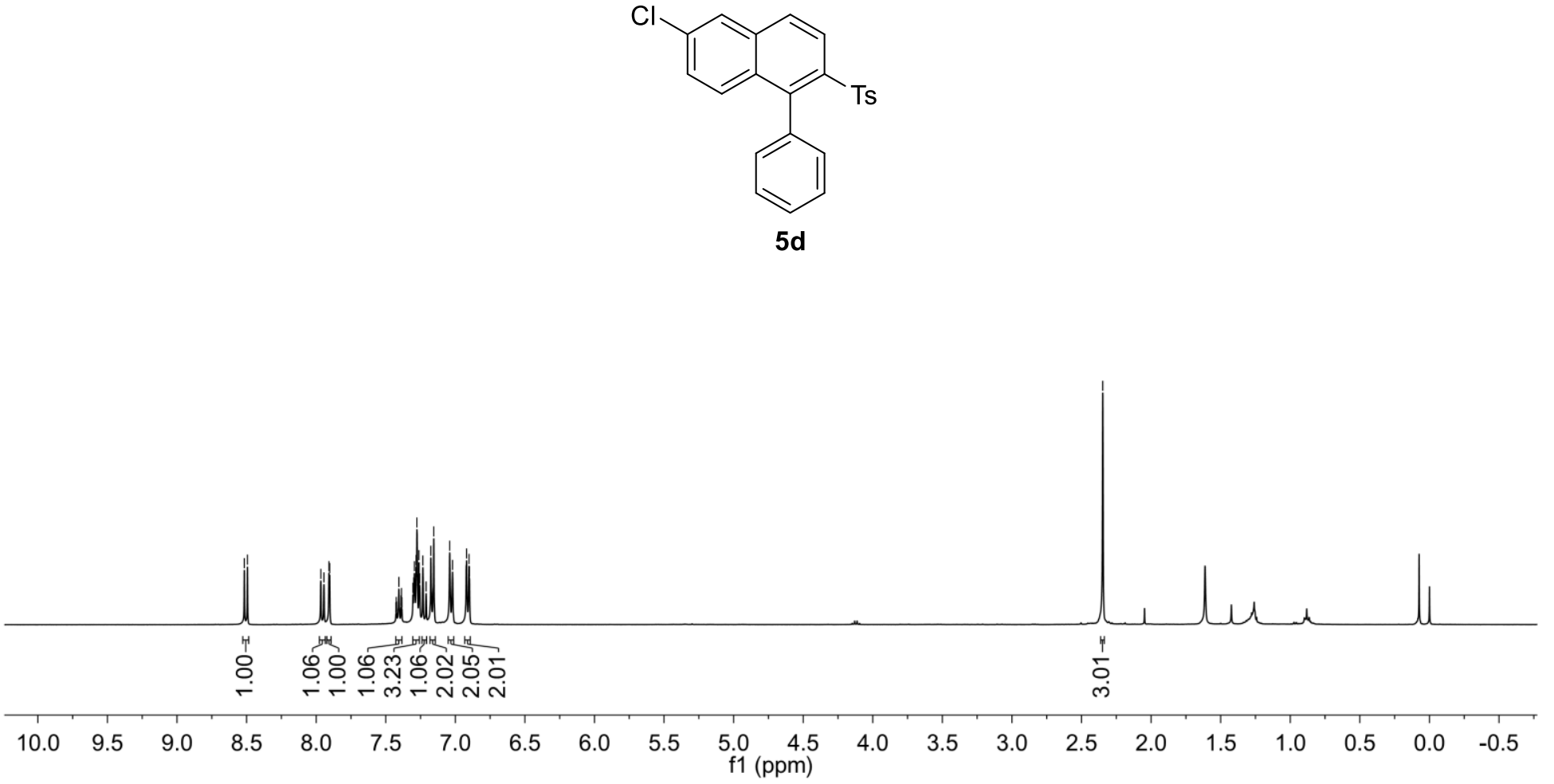

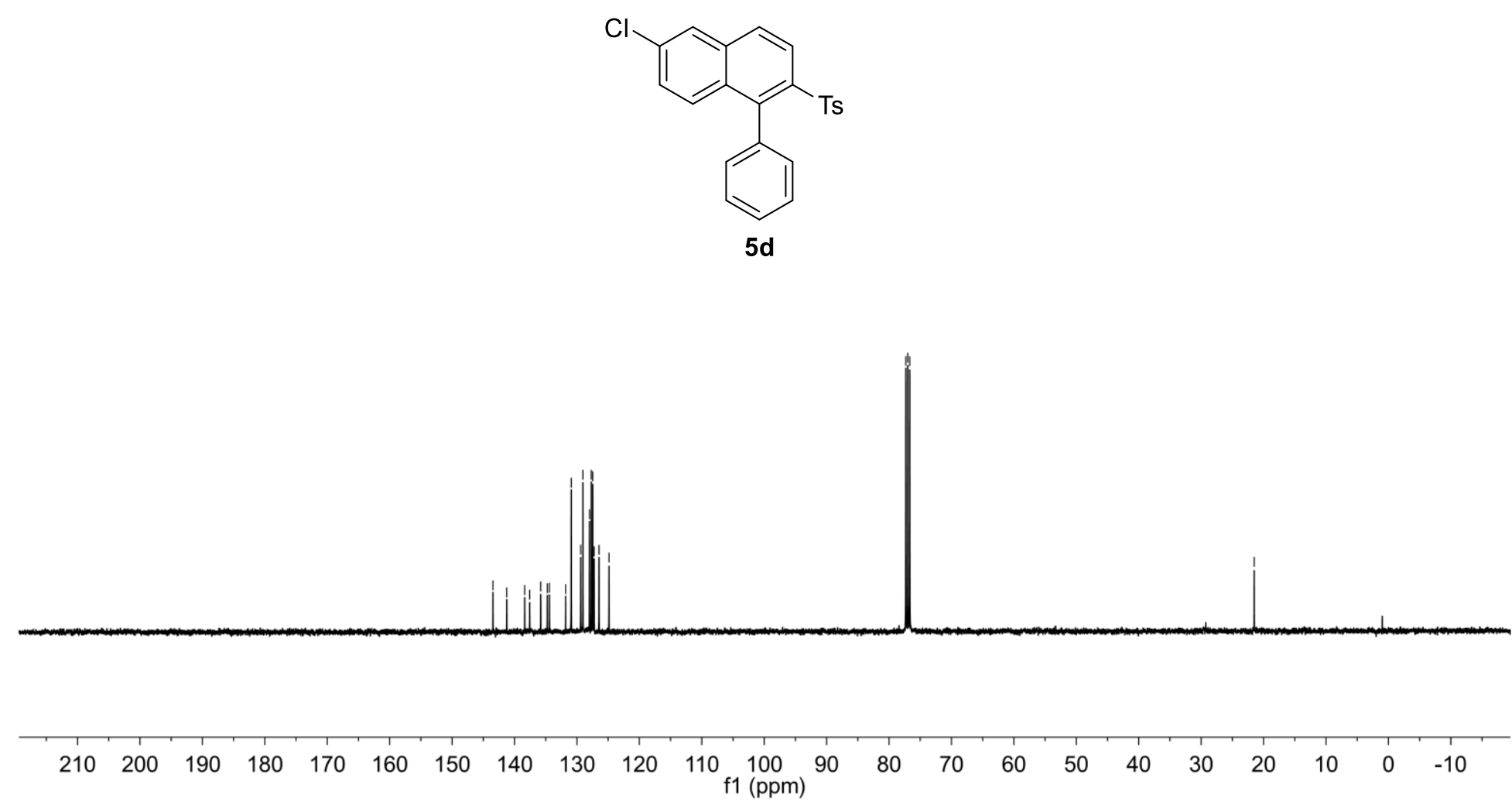


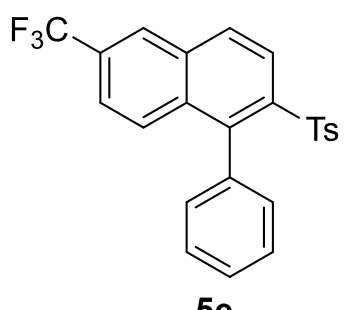

$5 e$

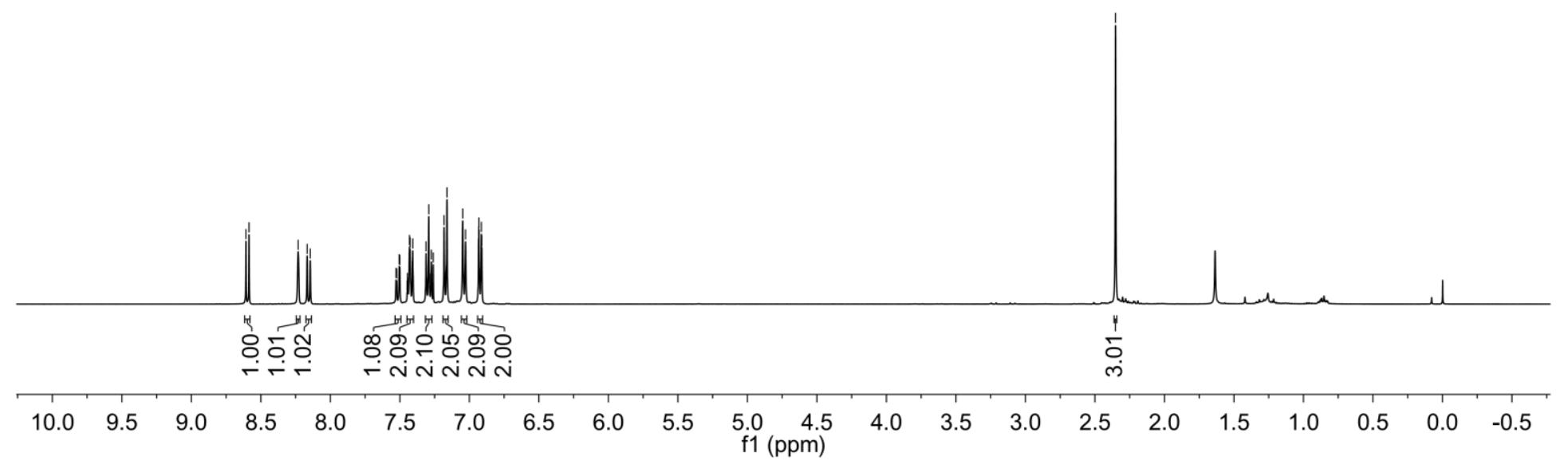




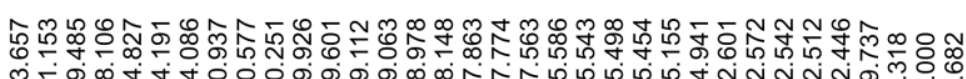

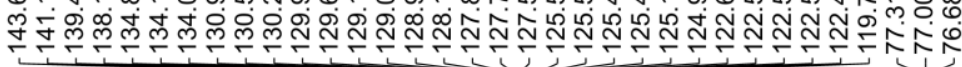
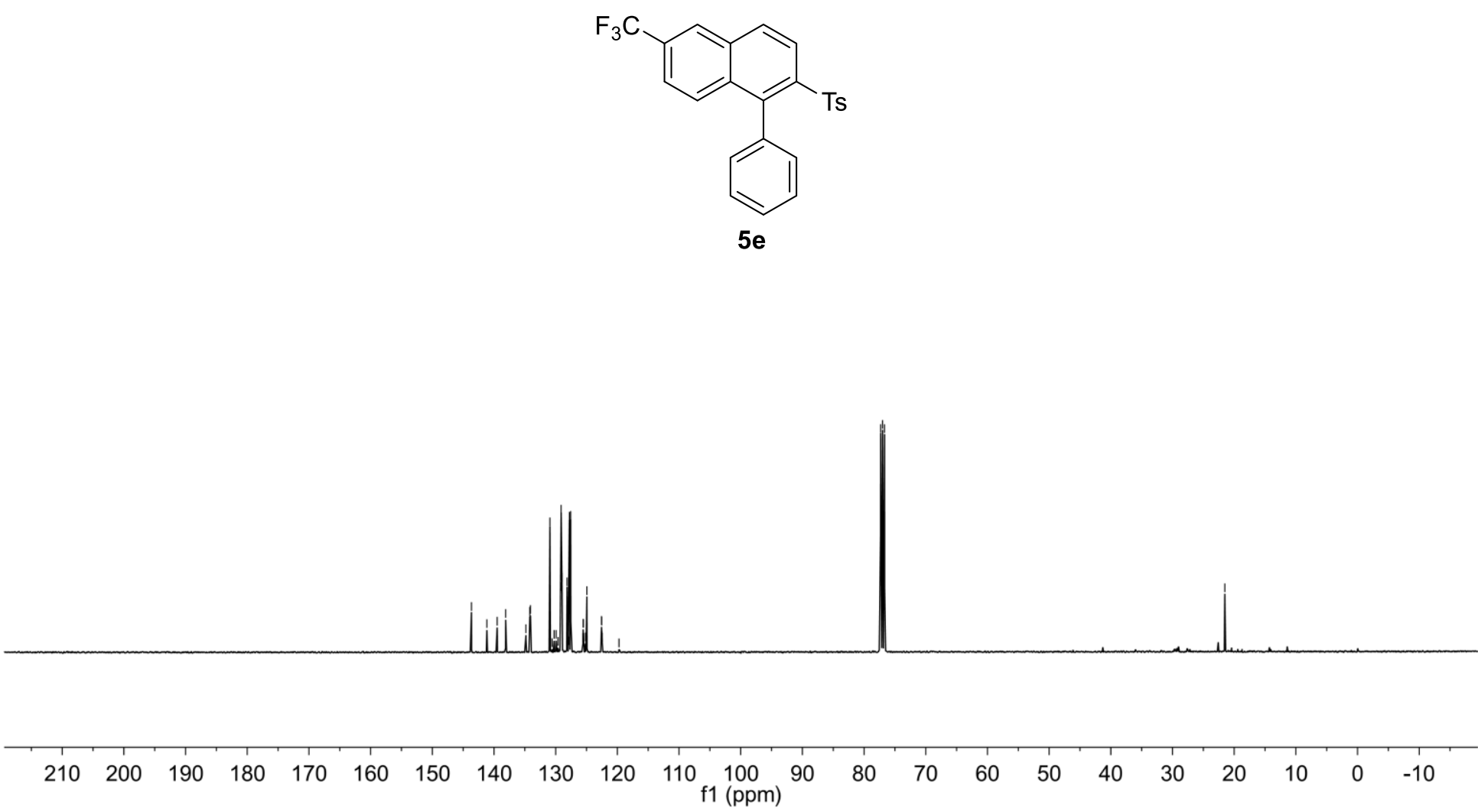

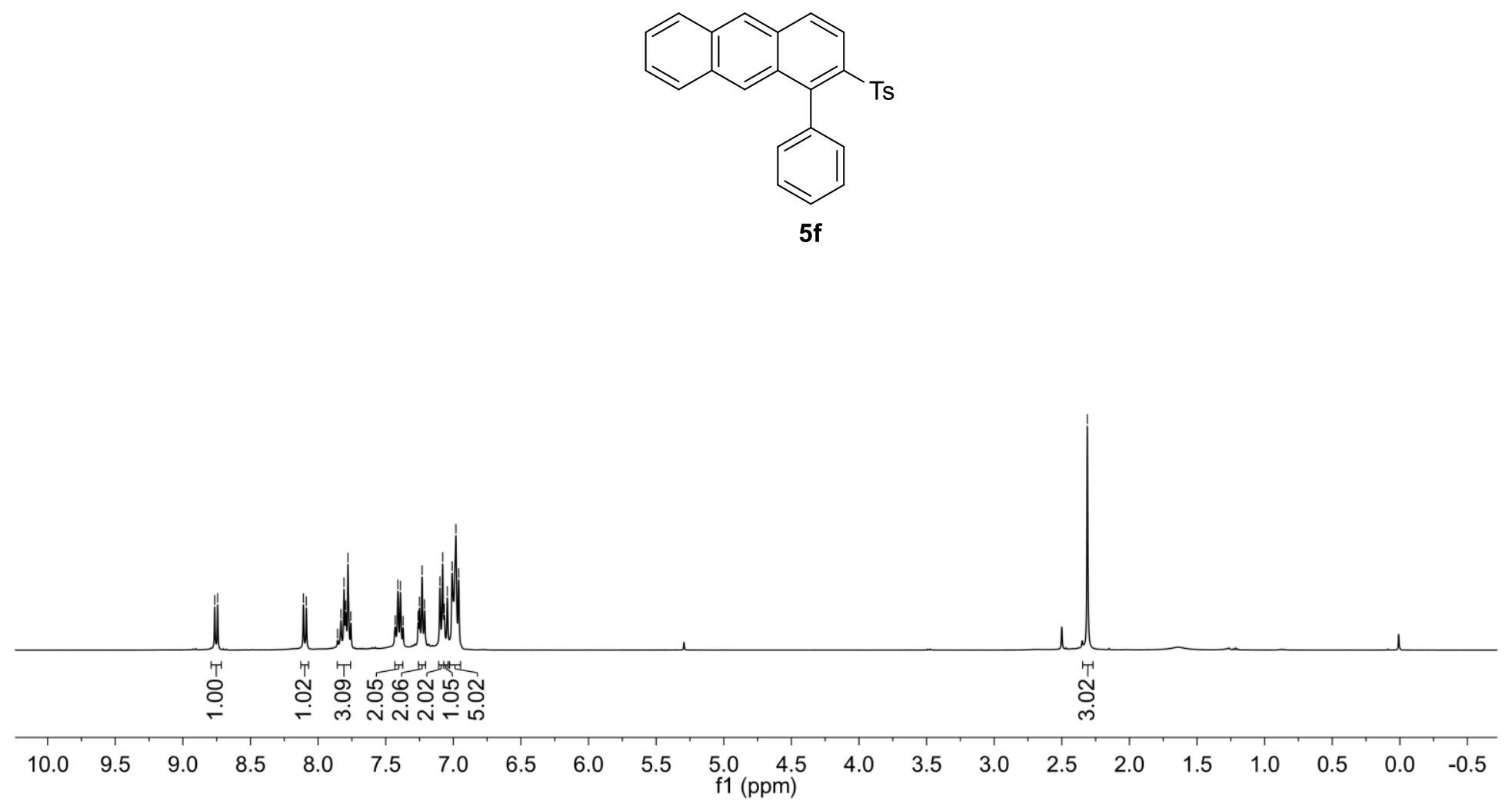

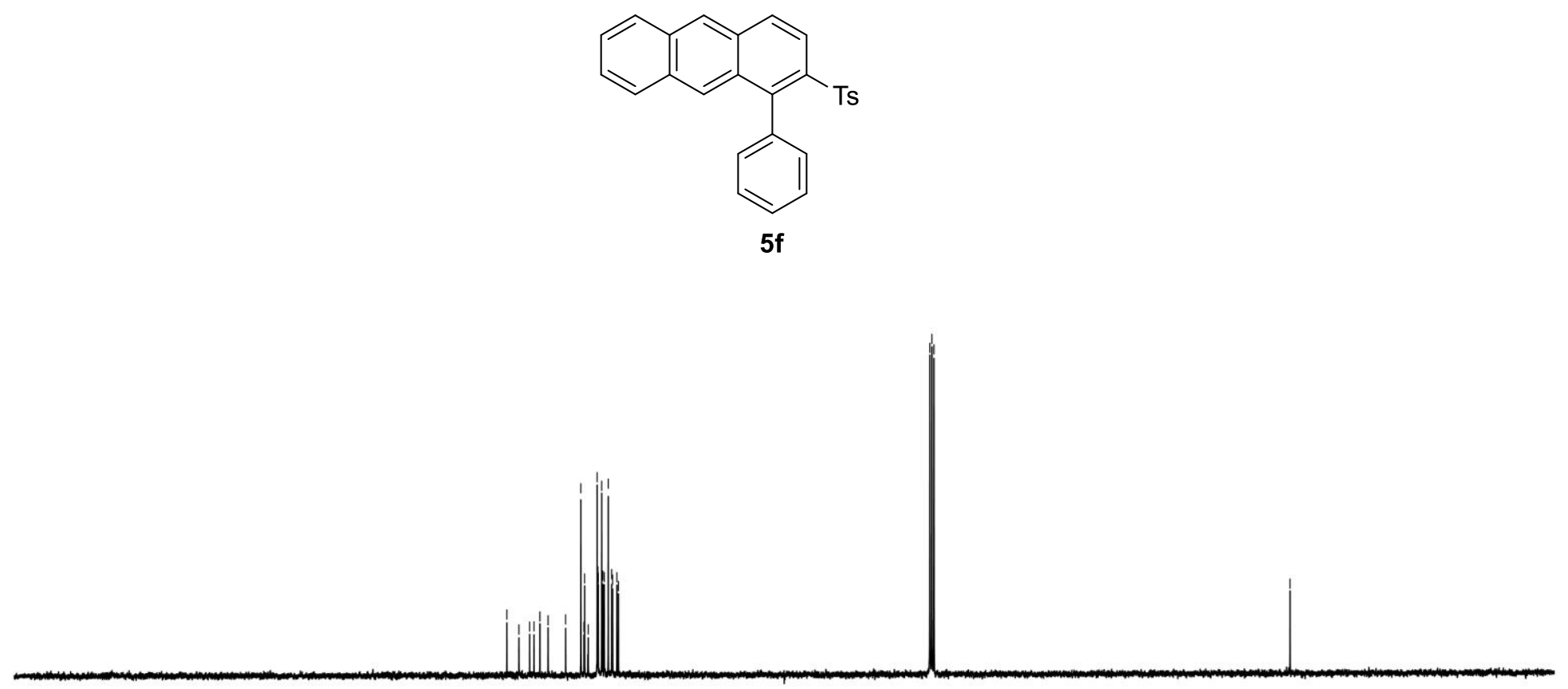

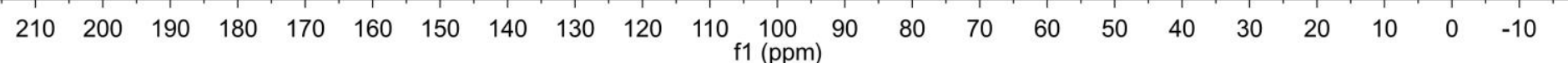



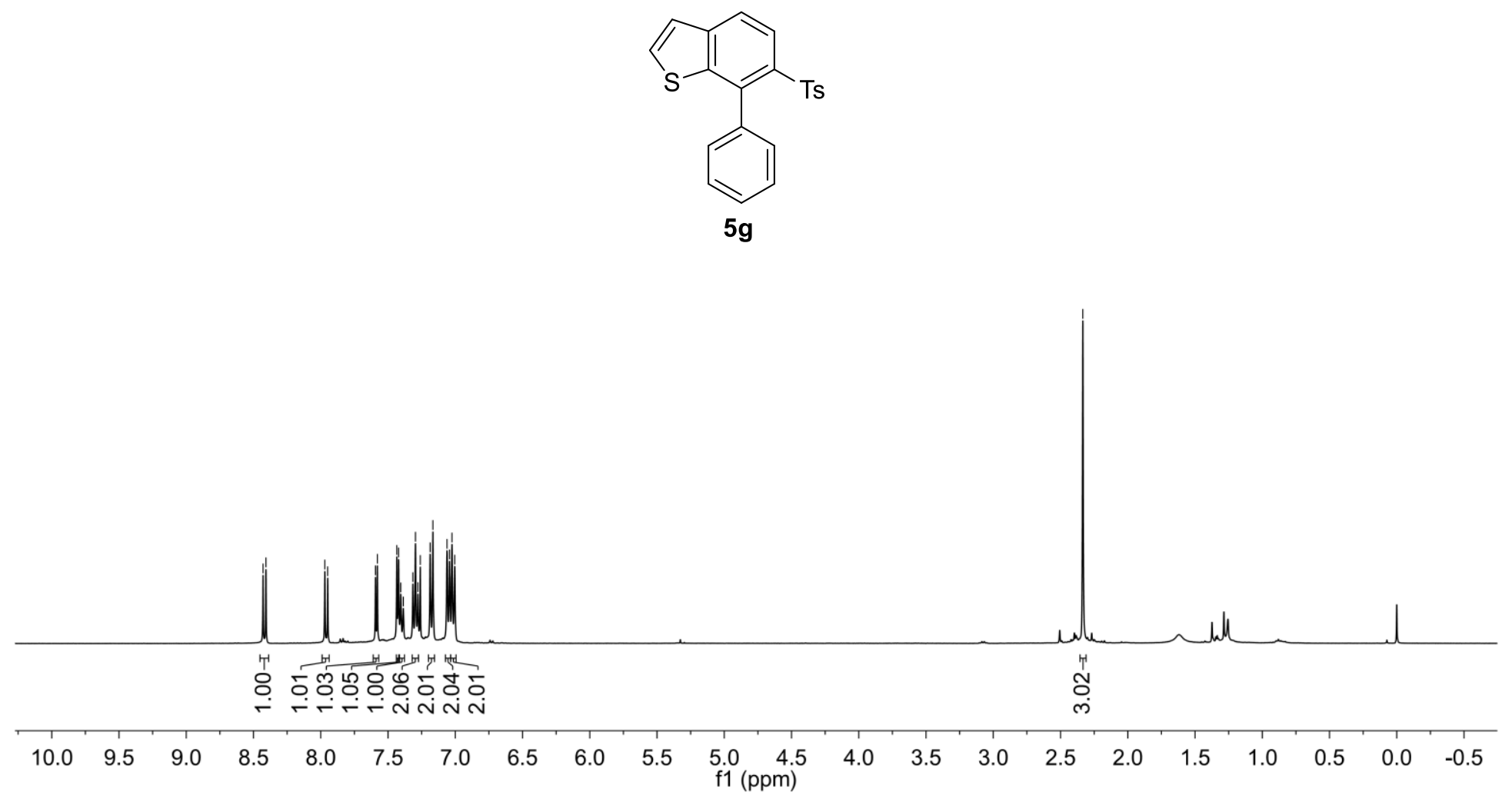


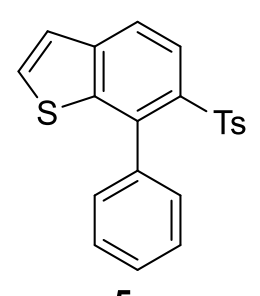

$5 \mathrm{~g}$

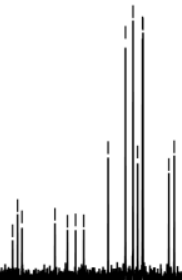

$\begin{array}{lllllllllll}210 & 200 & 190 & 180 & 170 & 160 & 150 & 140 & 130 & 120 & 110 \begin{array}{l}100 \\ \mathrm{f} 1(\mathrm{ppm})\end{array}\end{array}$ 


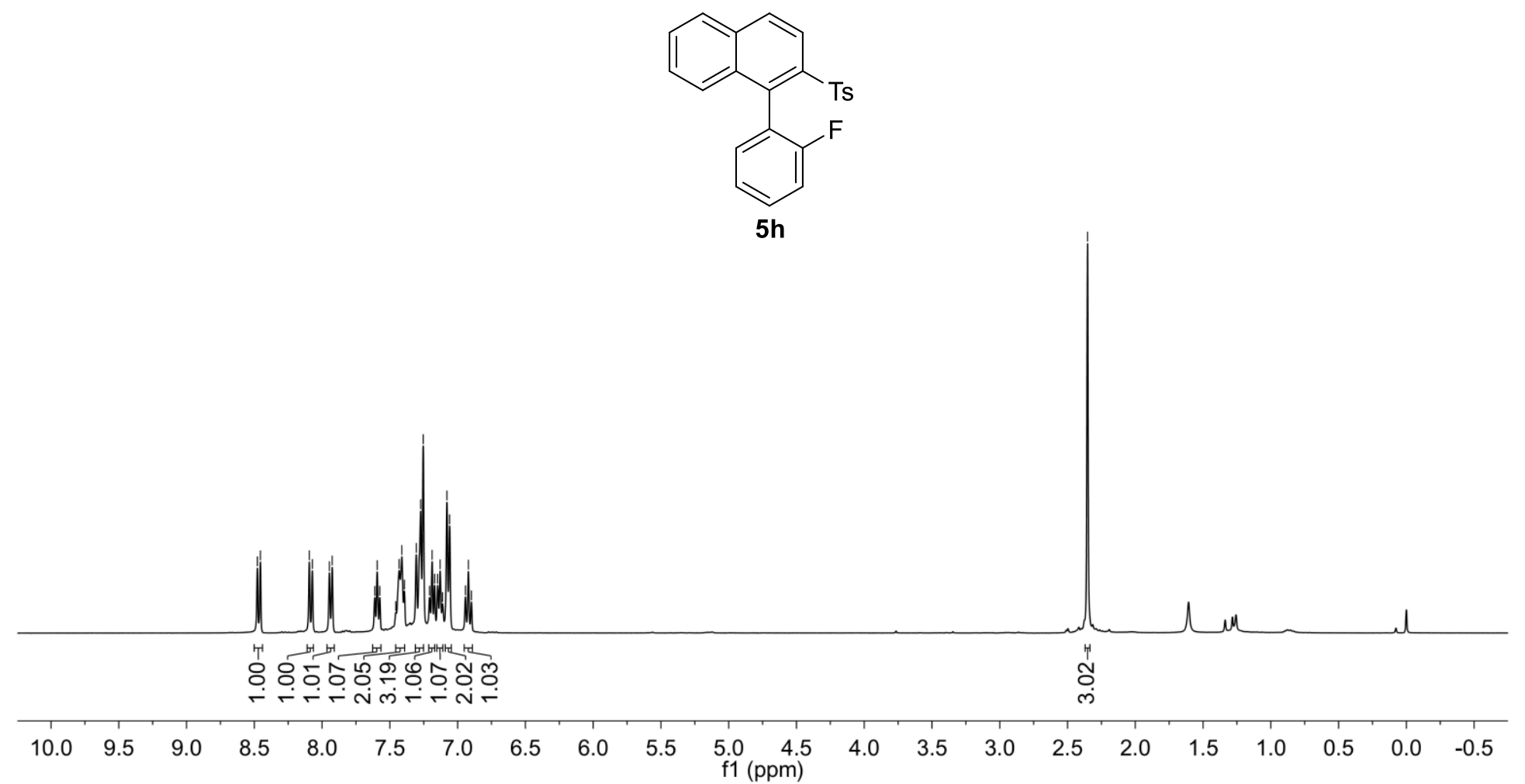




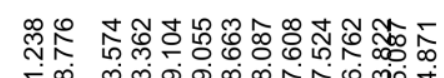

ঢํํำ

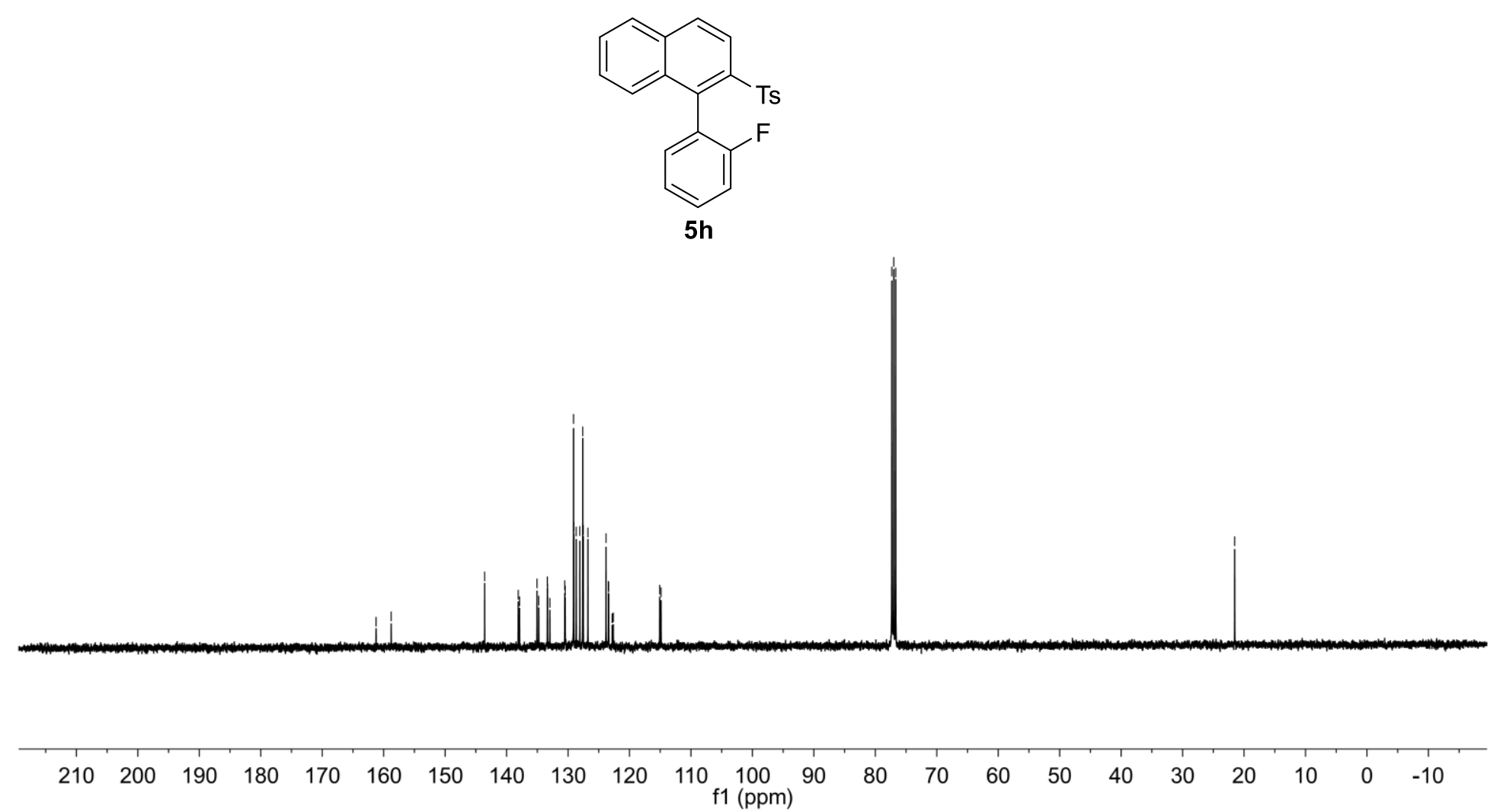




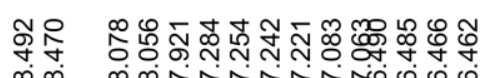

(9)
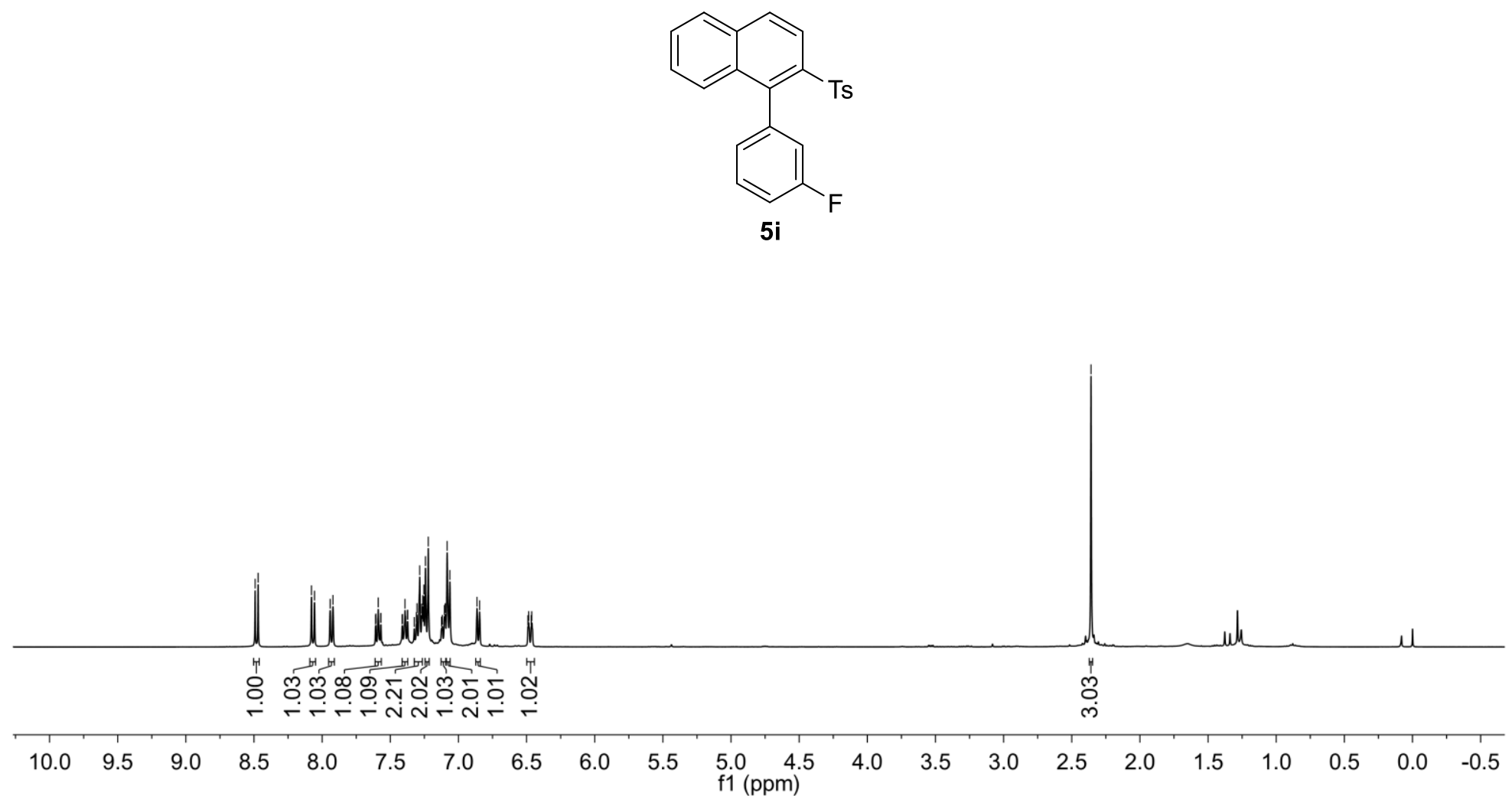


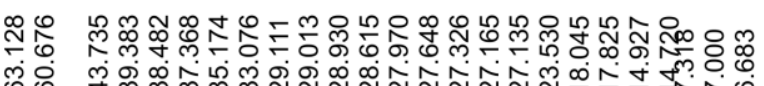

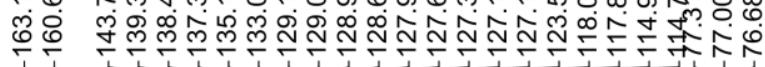
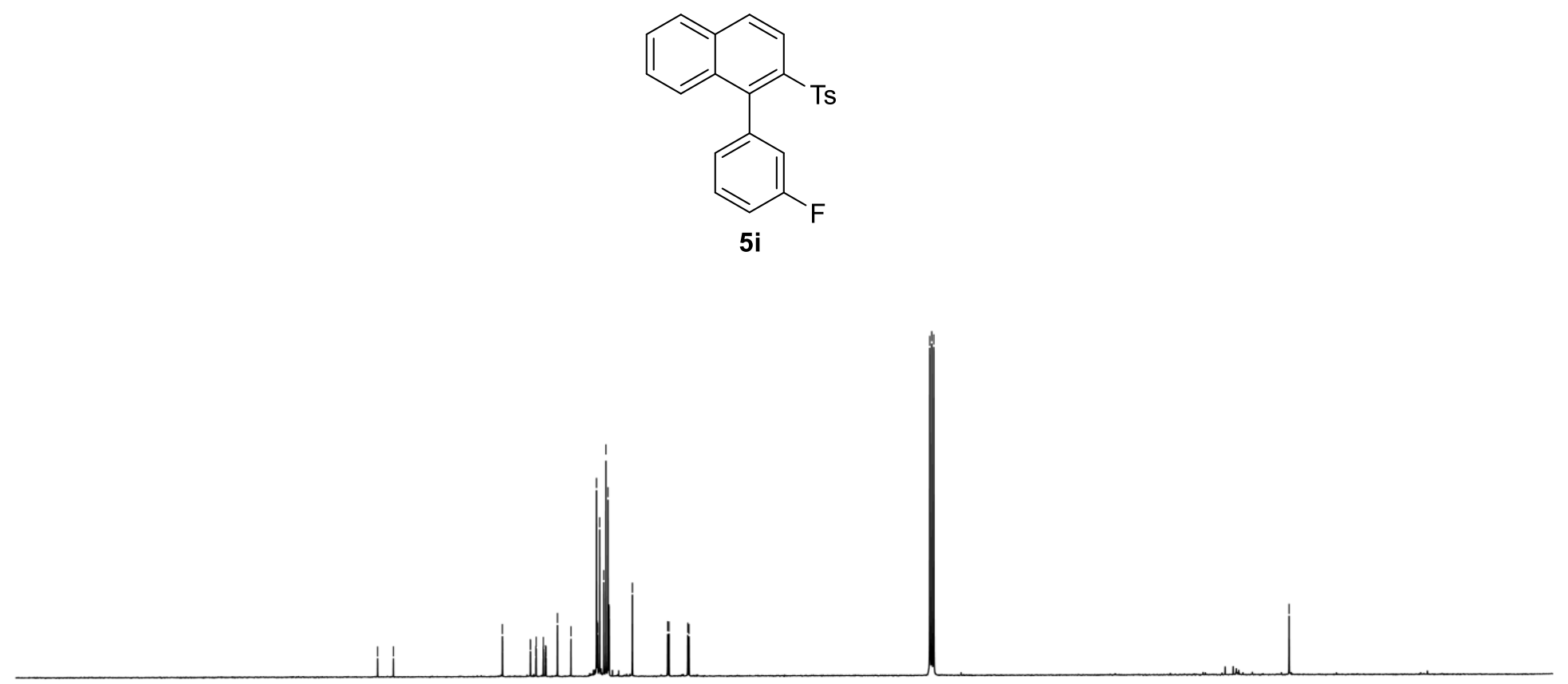

$\begin{array}{llllllllllll}210 & 200 & 190 & 180 & 170 & 160 & 150 & 140 & 130 & 120 & 110 & 100\end{array}$ f1 (ppm) 


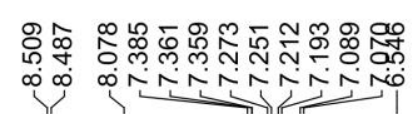
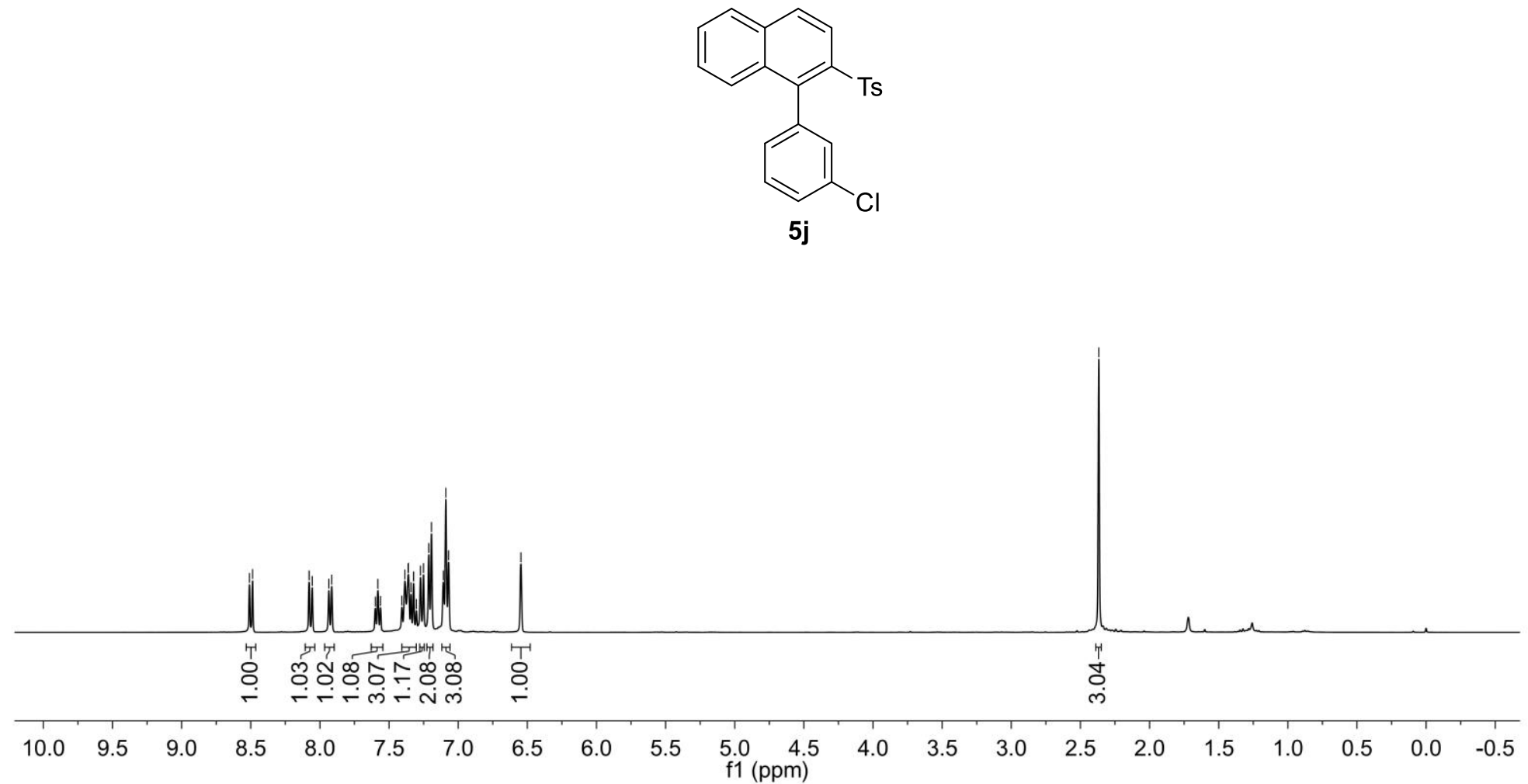

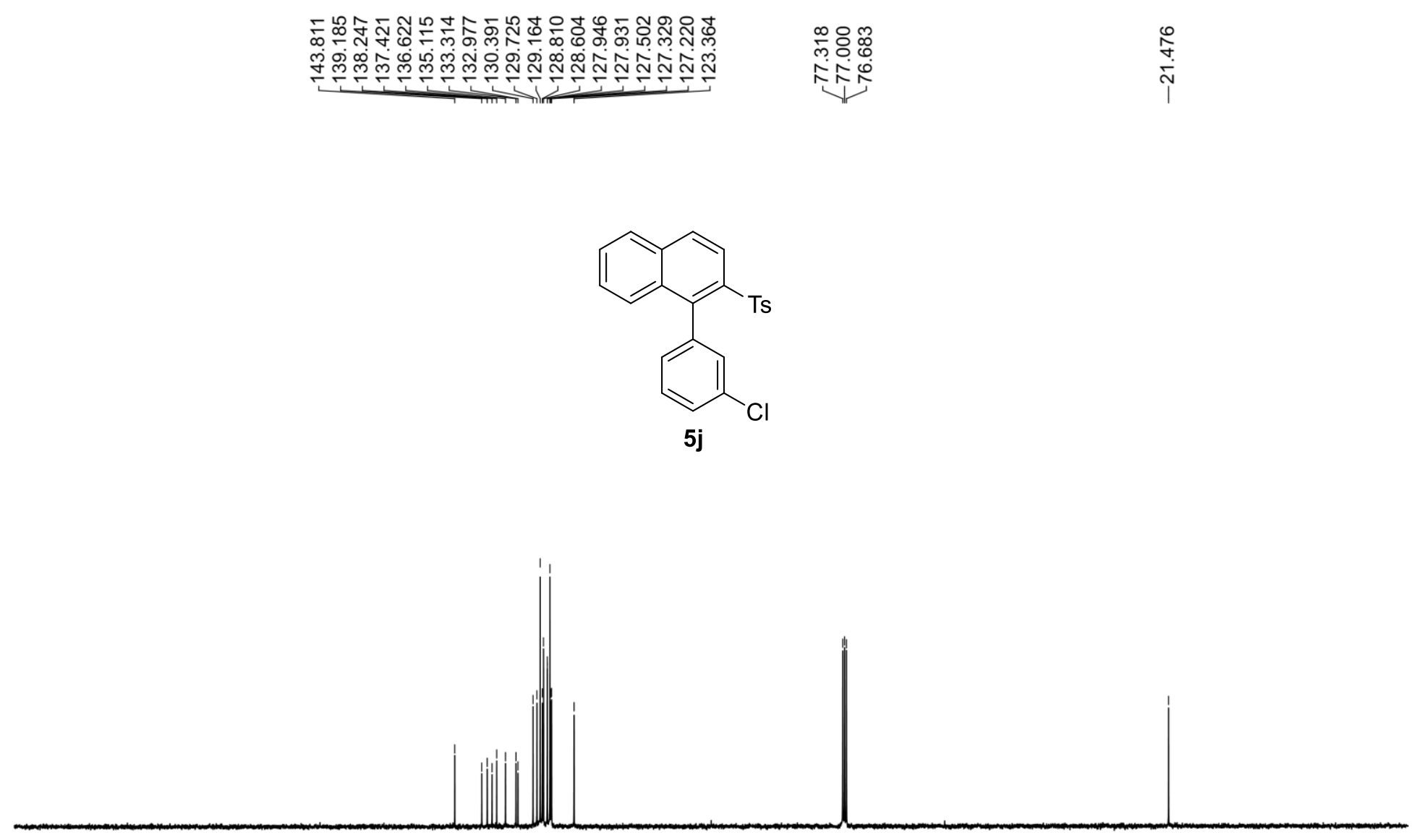

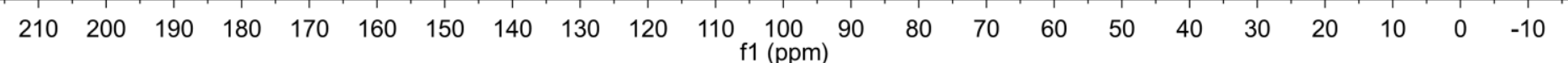


م

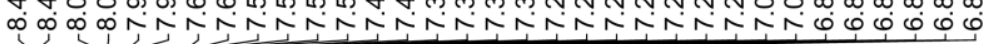

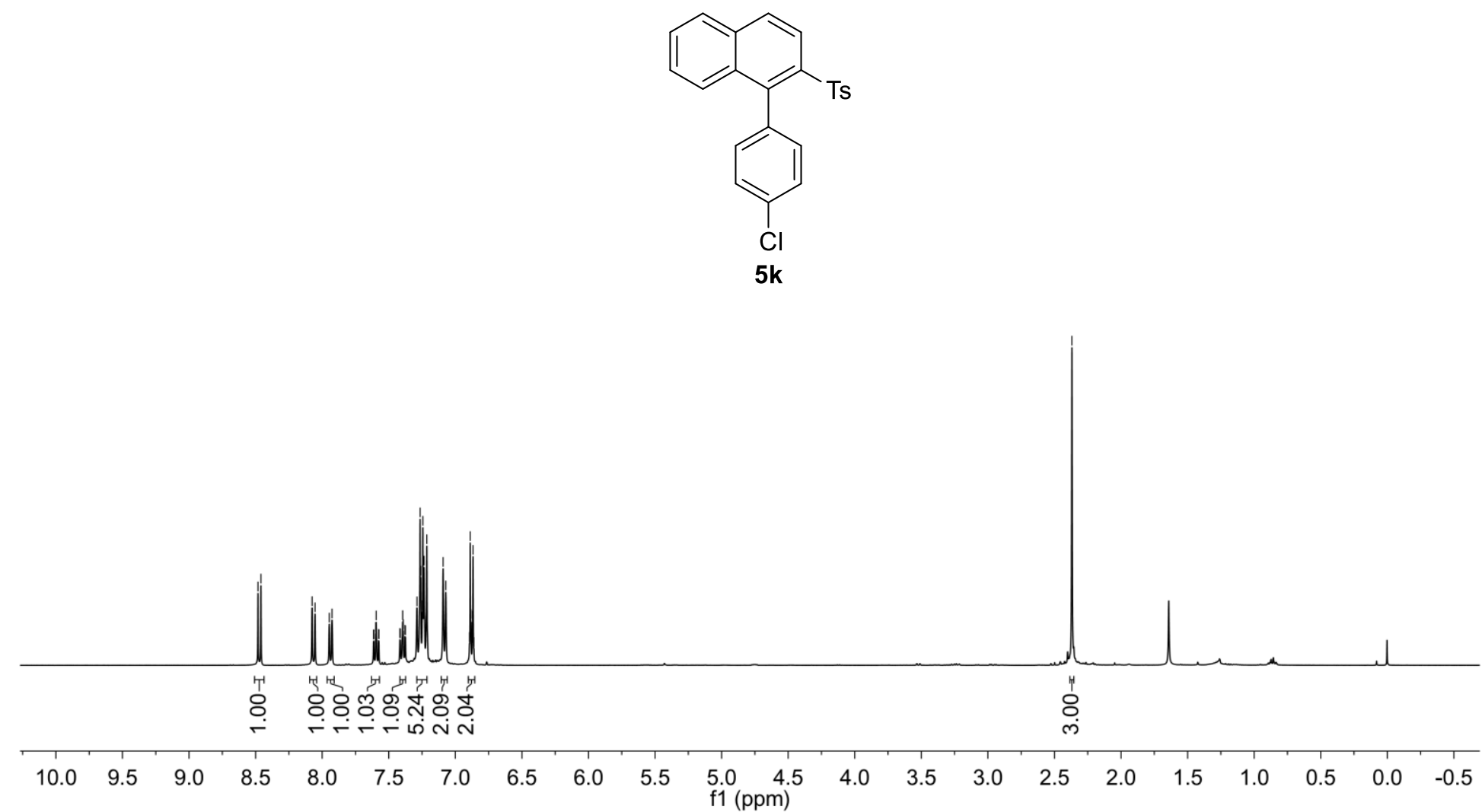



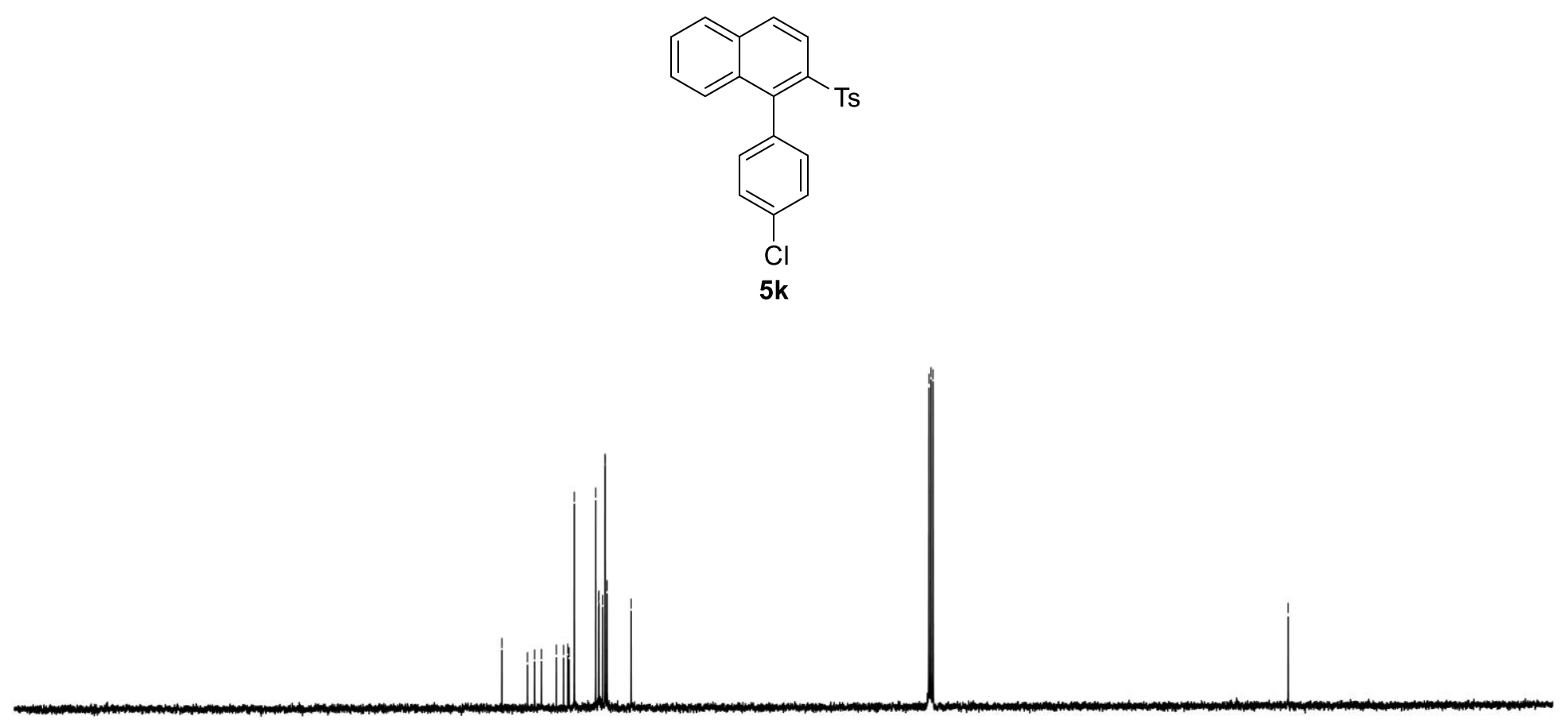

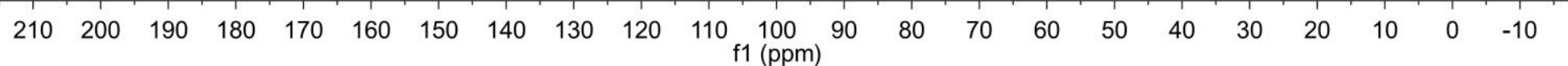




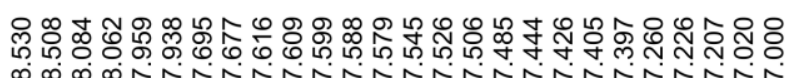

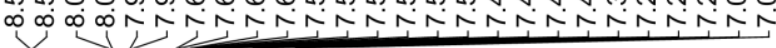

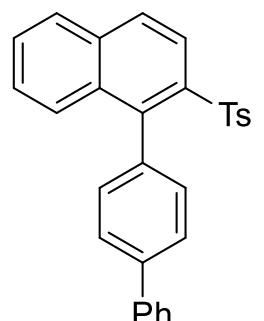

5

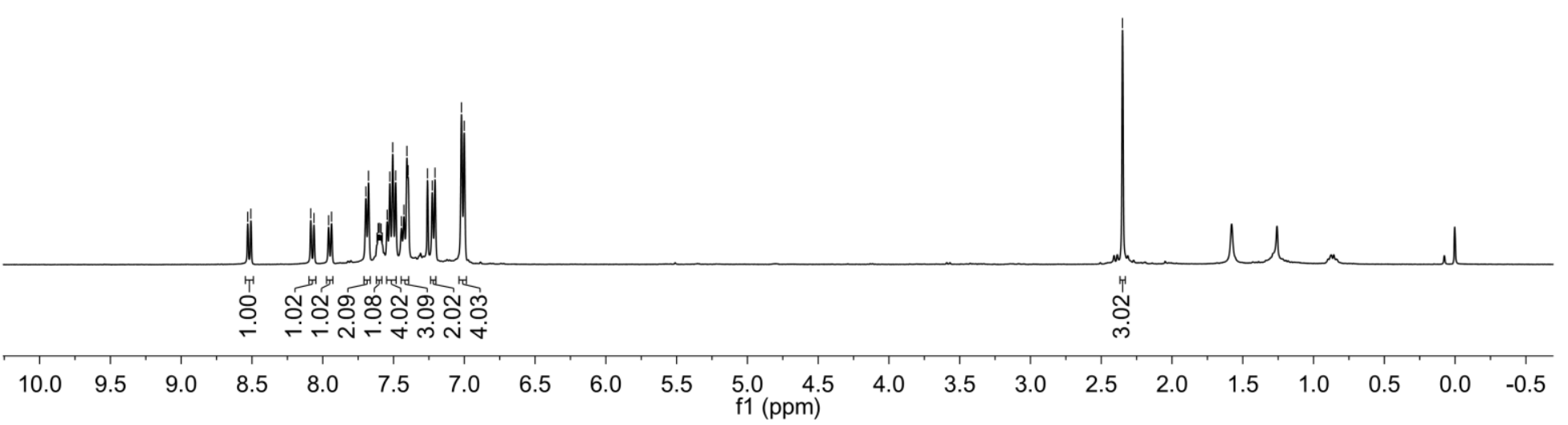




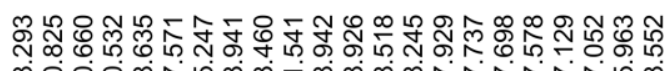

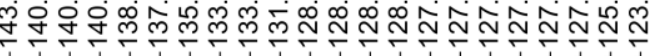
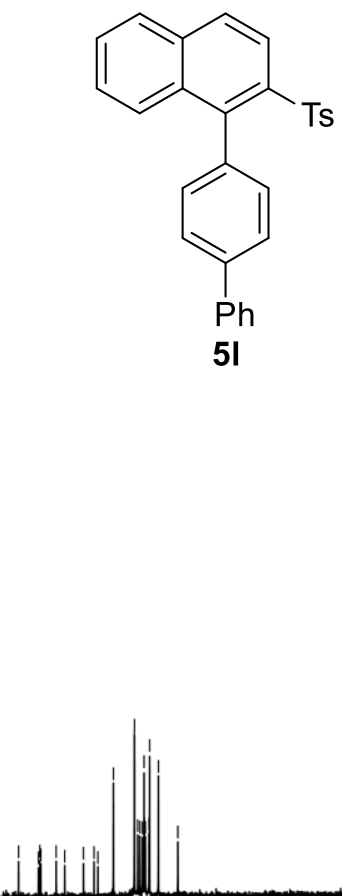

$\begin{array}{lllllllllll}210 & 200 & 190 & 180 & 170 & 160 & 150 & 140 & 130 & 120 & 110 \begin{array}{l}100 \\ \mathrm{f} 1(\mathrm{ppm})\end{array}\end{array}$ 


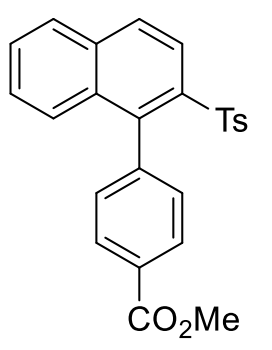

$5 \mathrm{~m}$

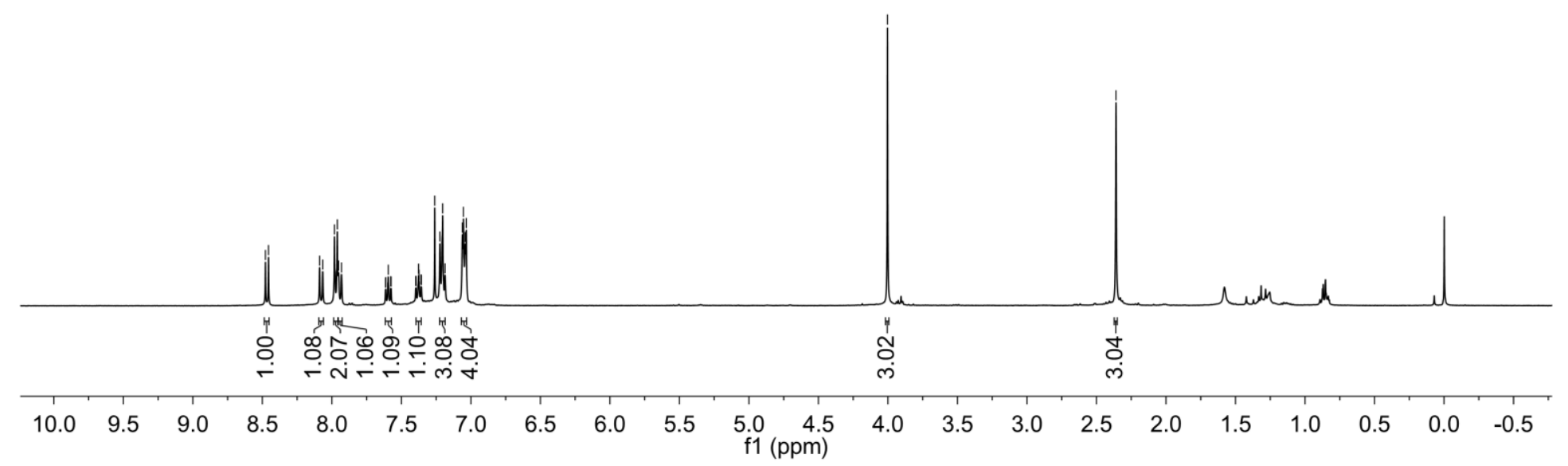




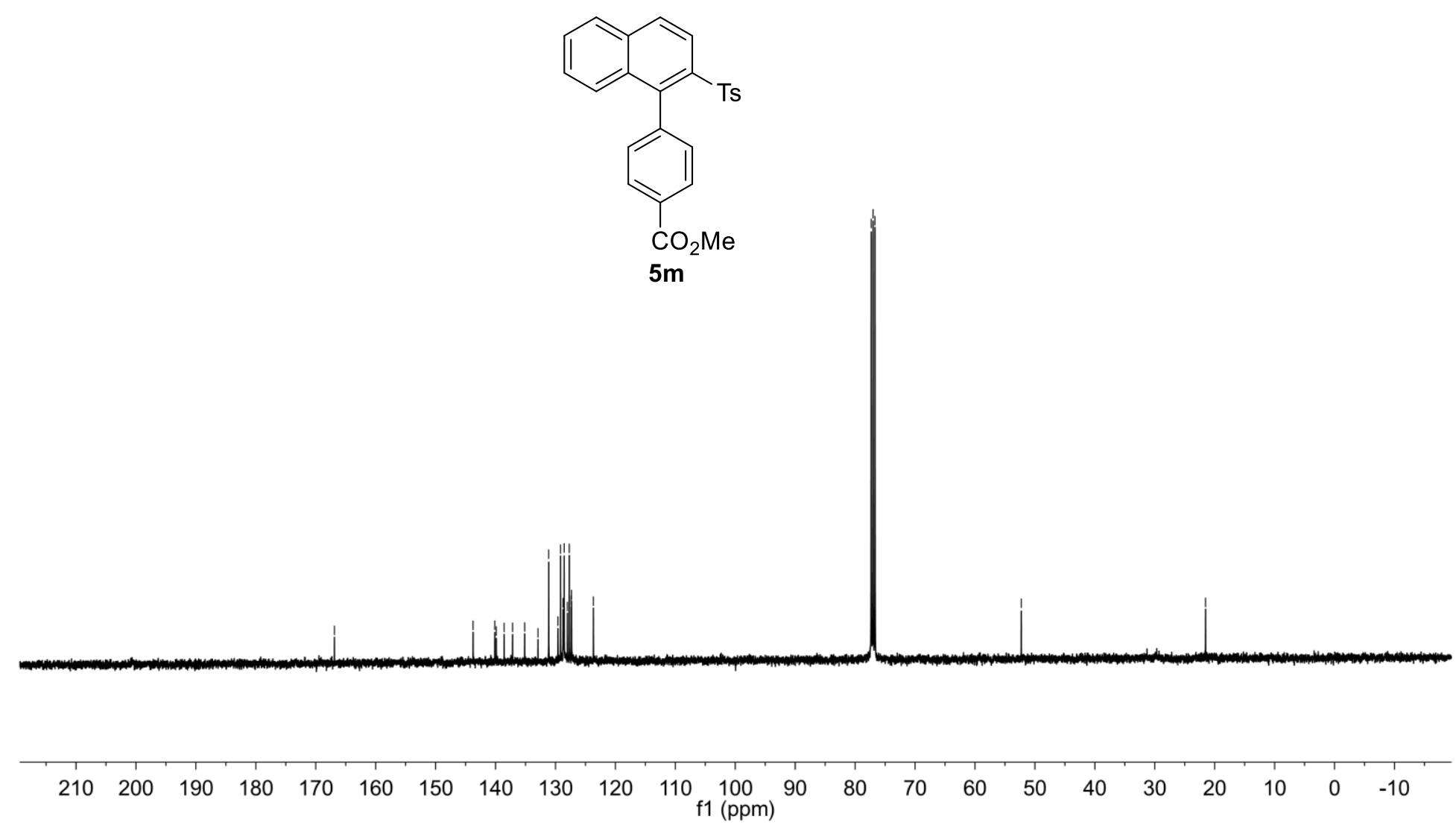




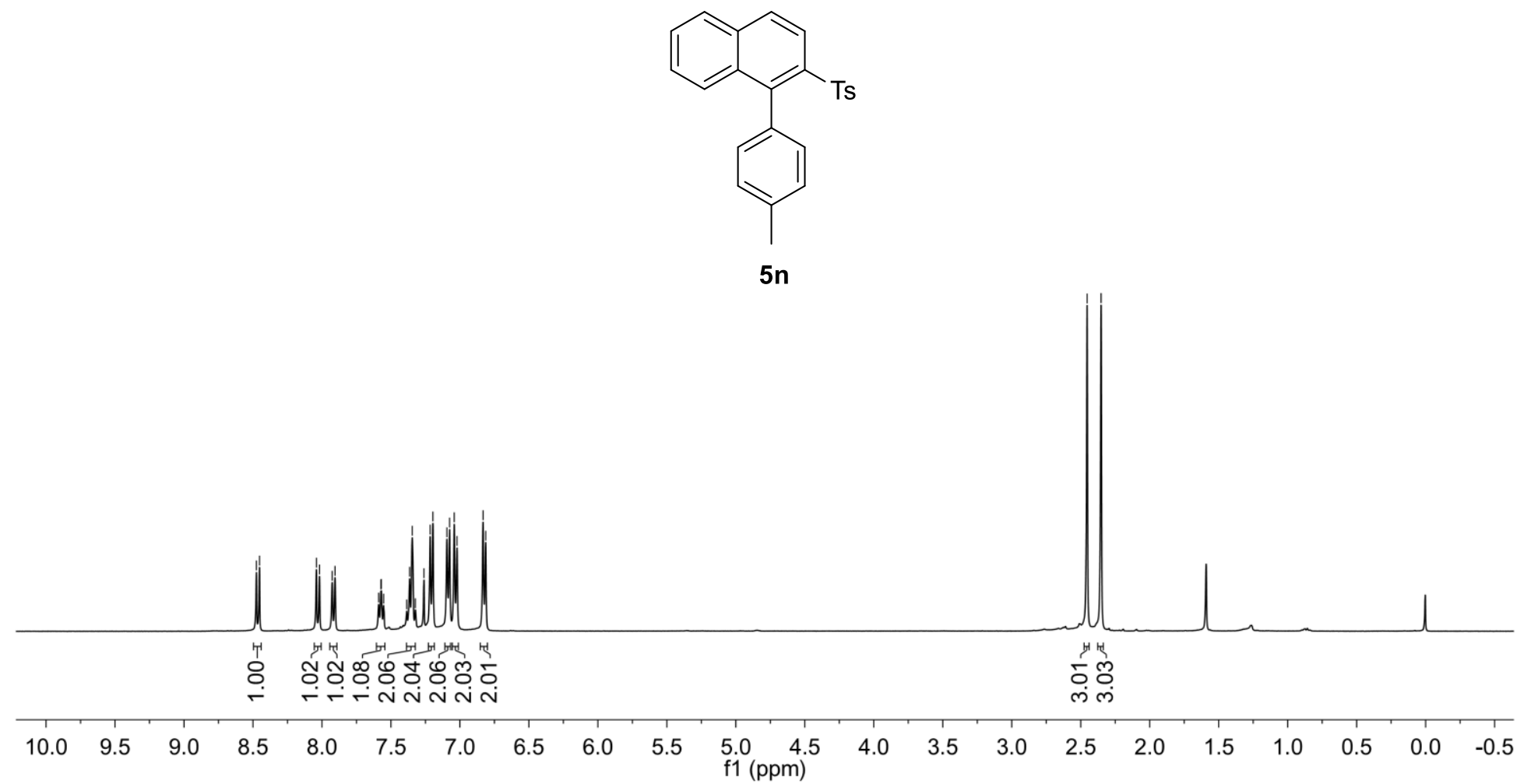




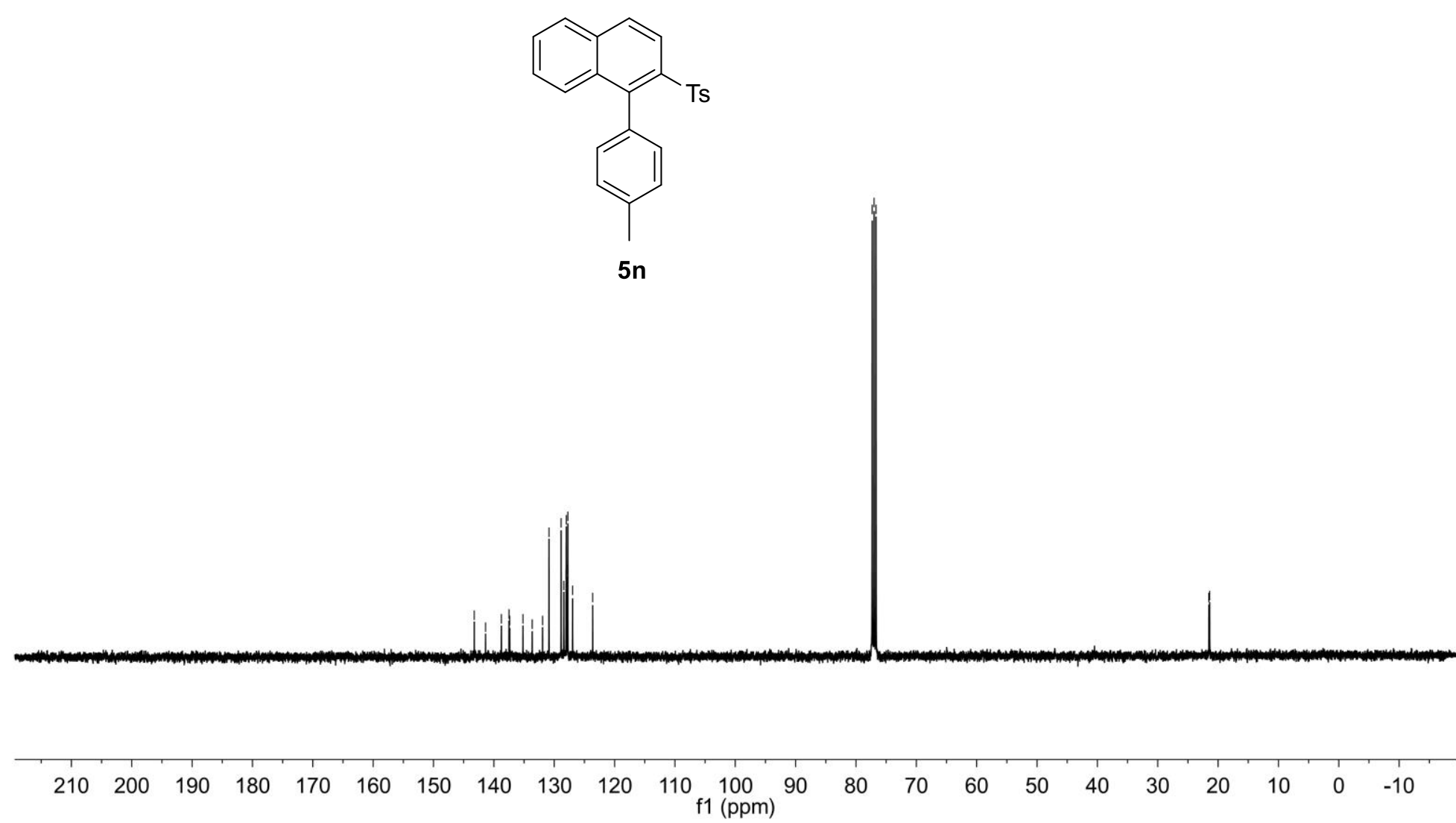




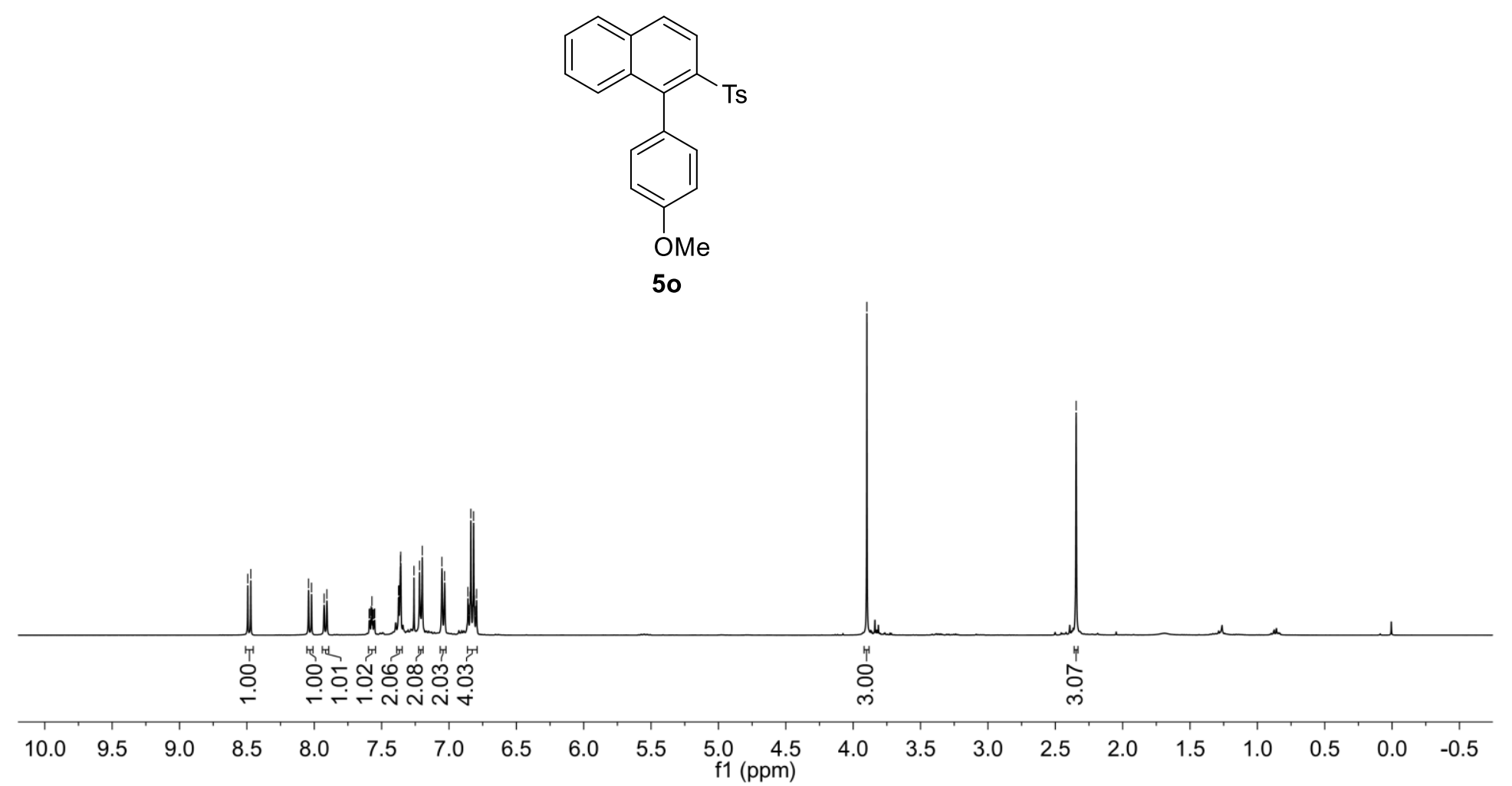




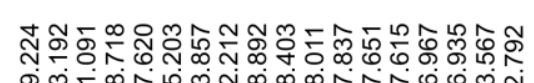

。ำ

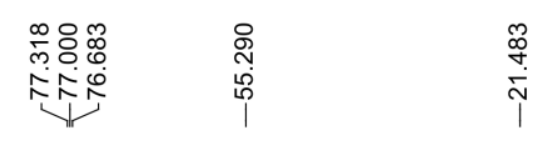

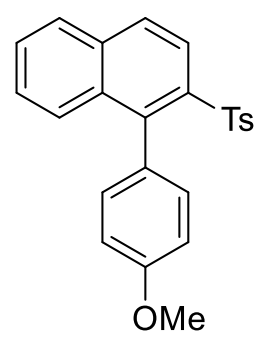

50

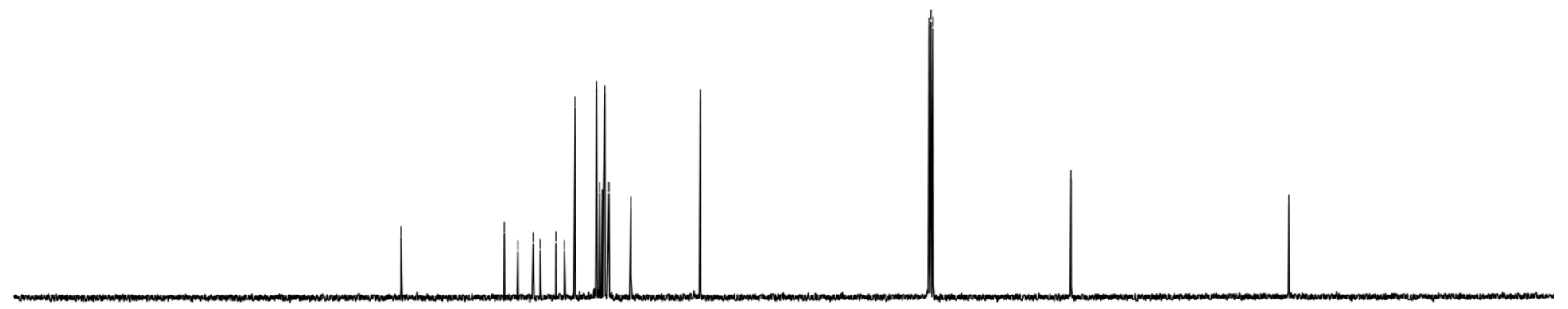

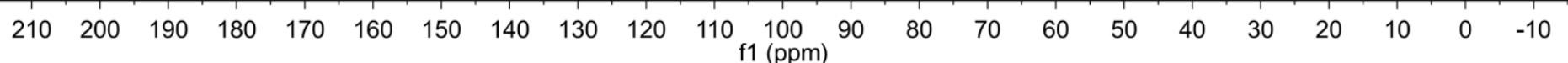



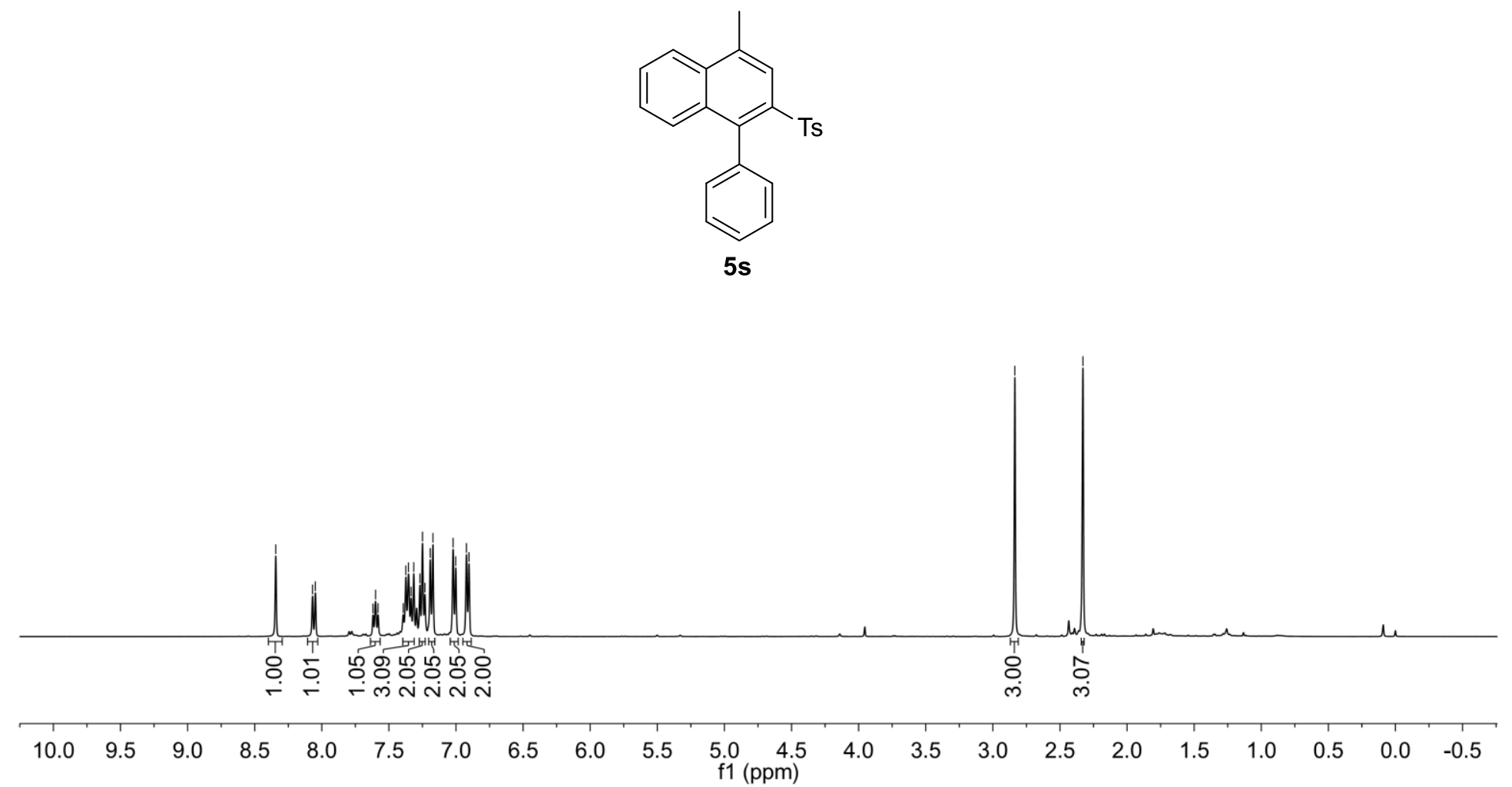

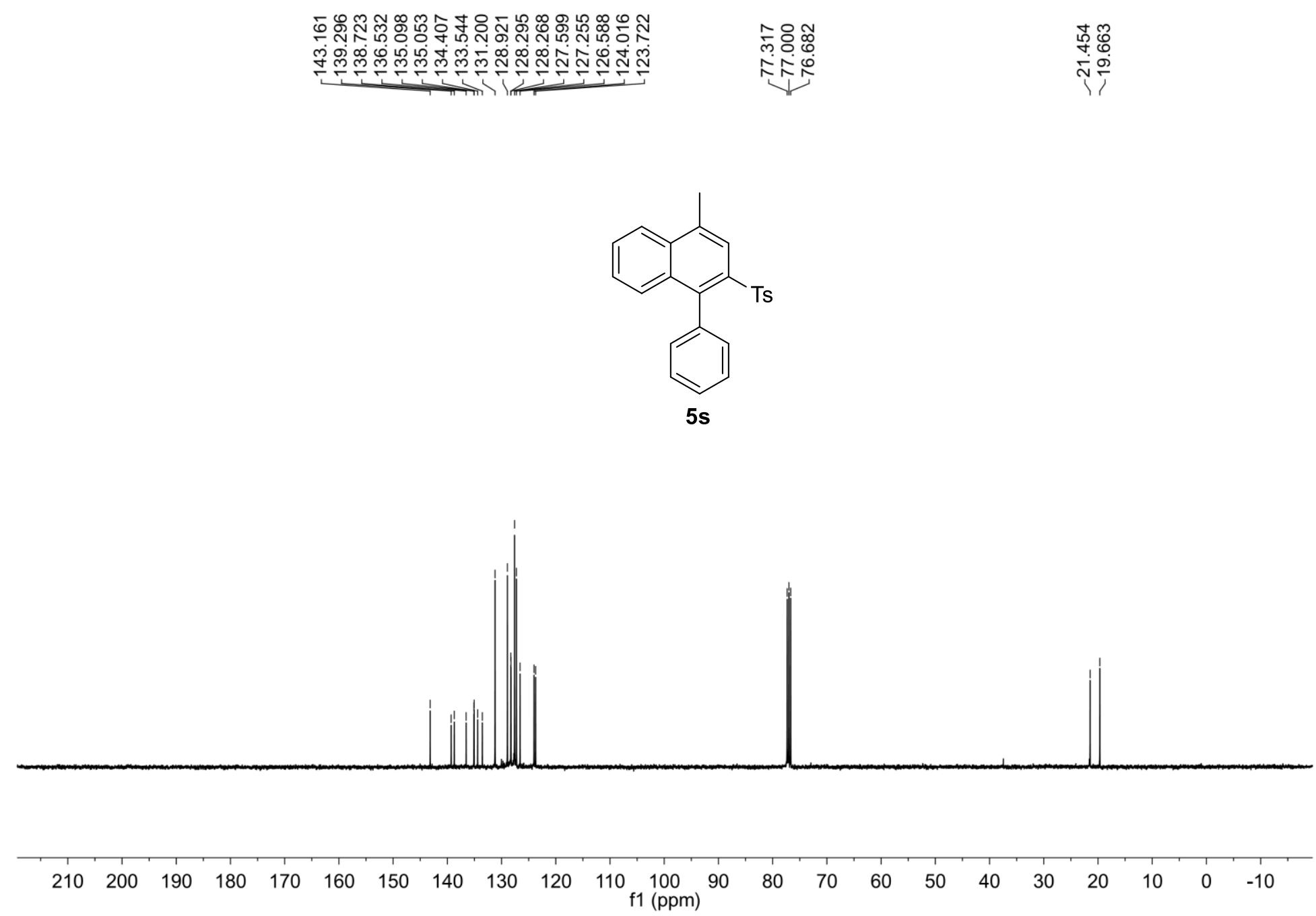


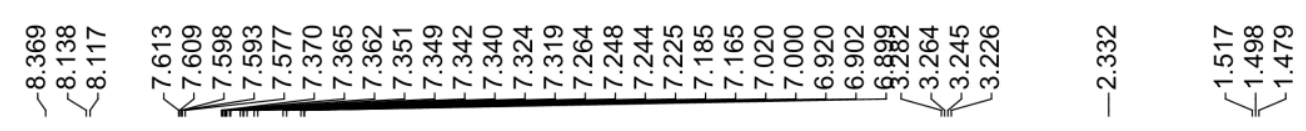
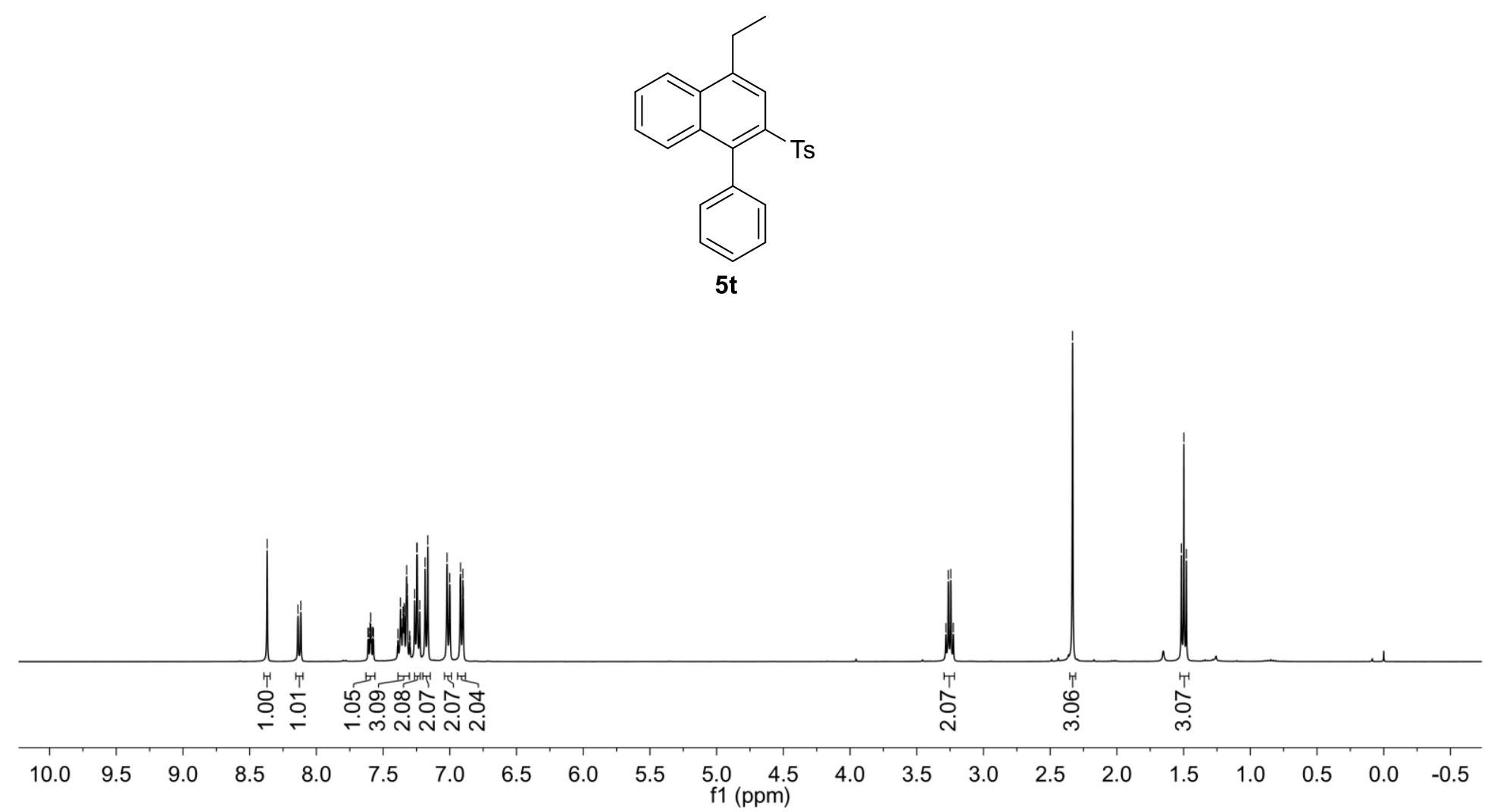

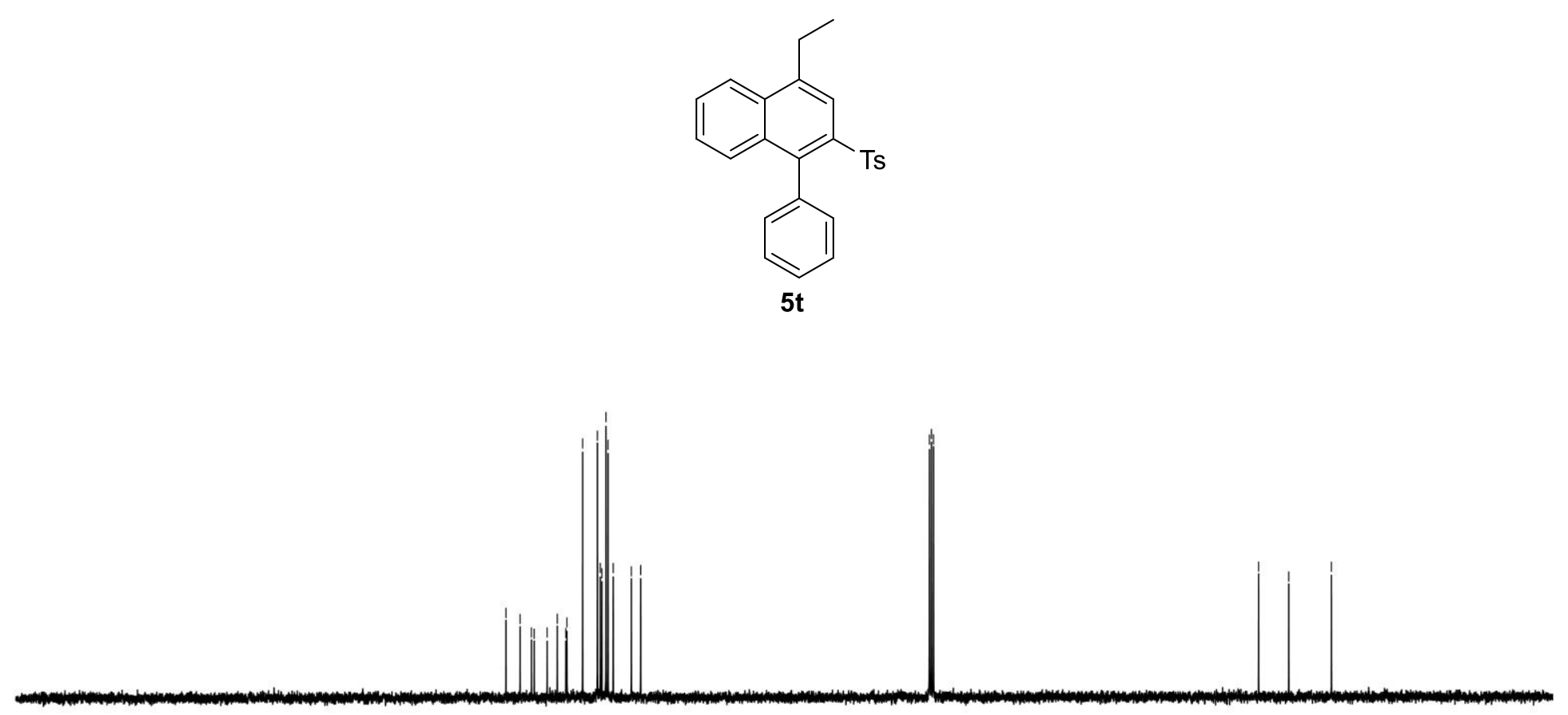

$\begin{array}{lllllllllllllllllllllll}210 & 200 & 190 & 180 & 170 & 160 & 150 & 140 & 130 & 120 & 110 & 100 & 90 & 80 & 70 & 60 & 50 & 40 & 30 & 20 & 10 & 0 & -10\end{array}$ 


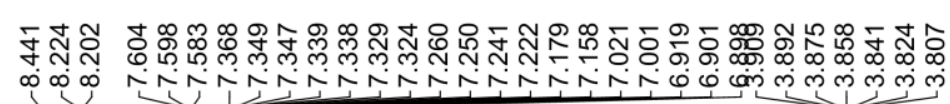

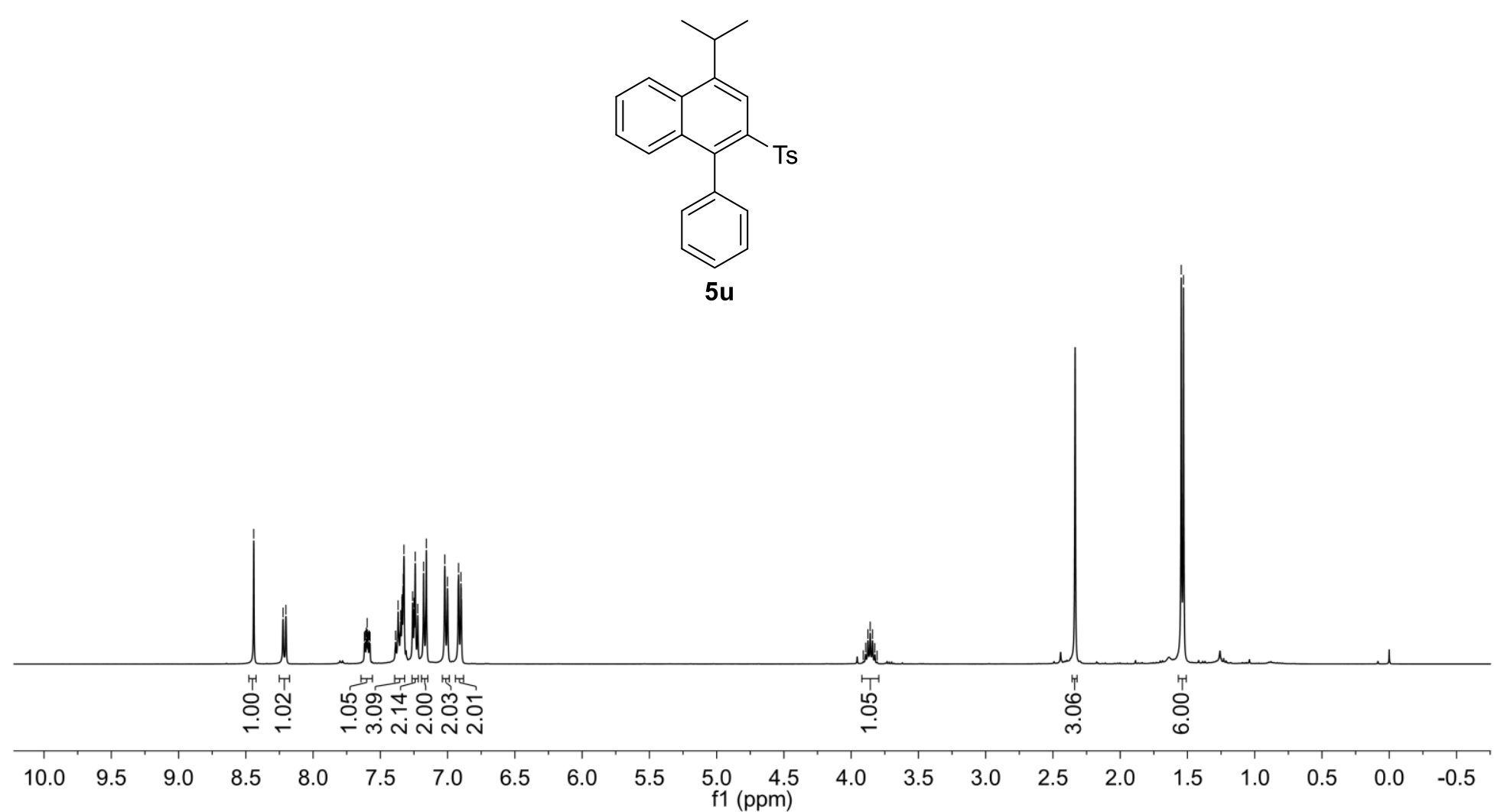

S114 

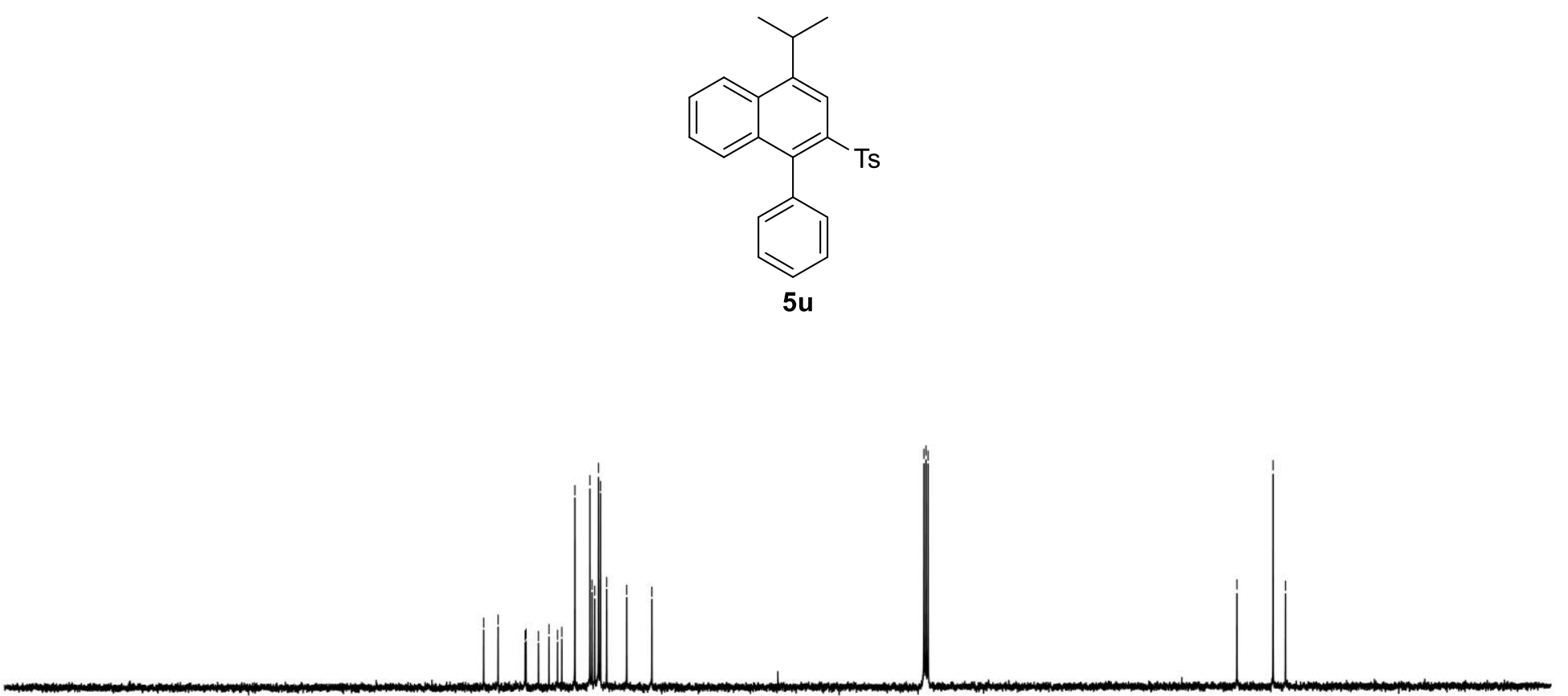

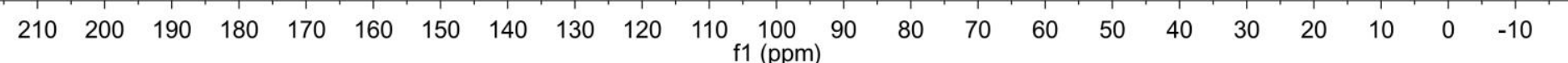




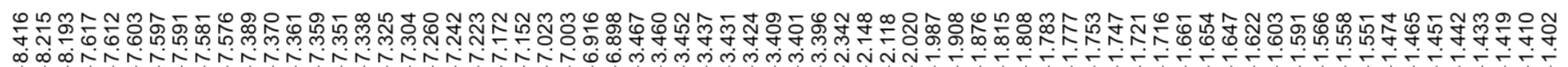

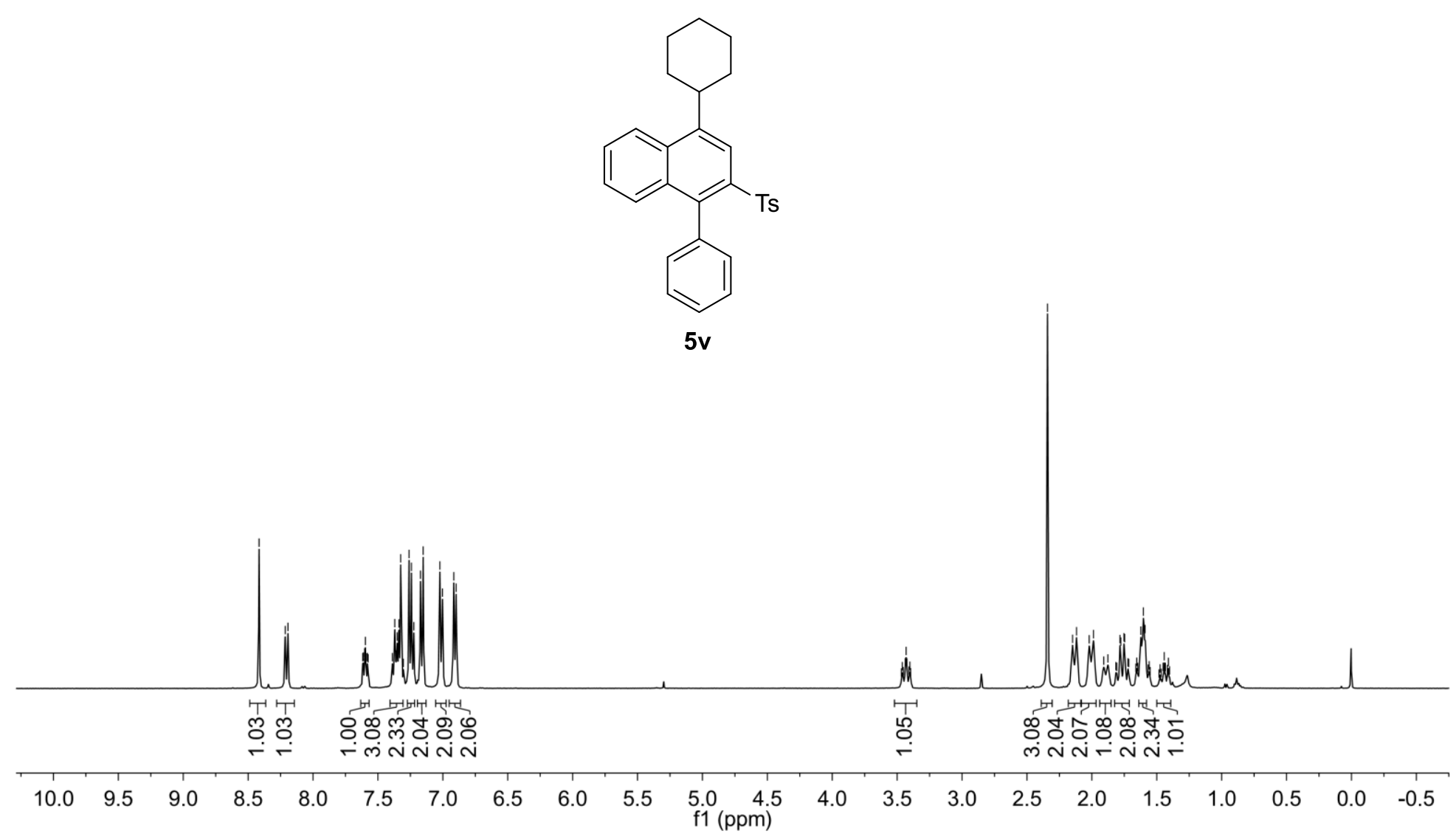

S116 

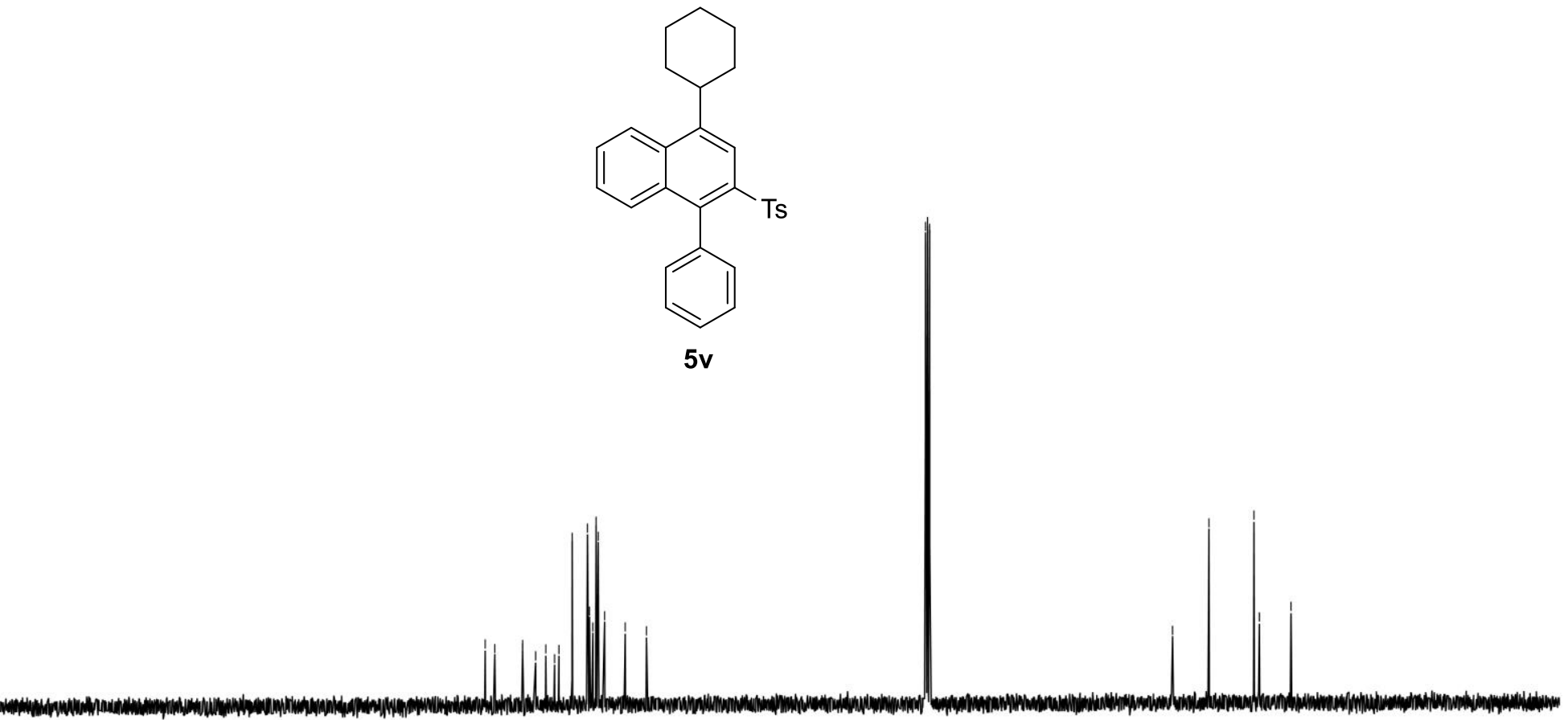

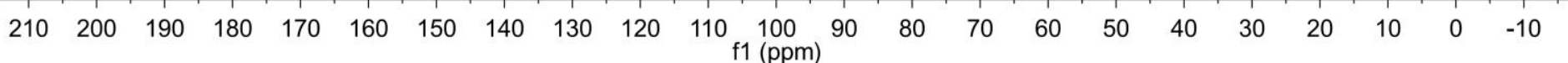




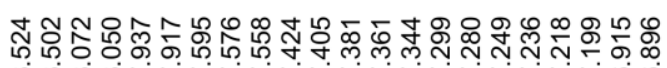

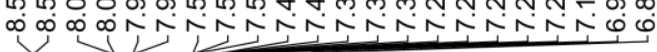
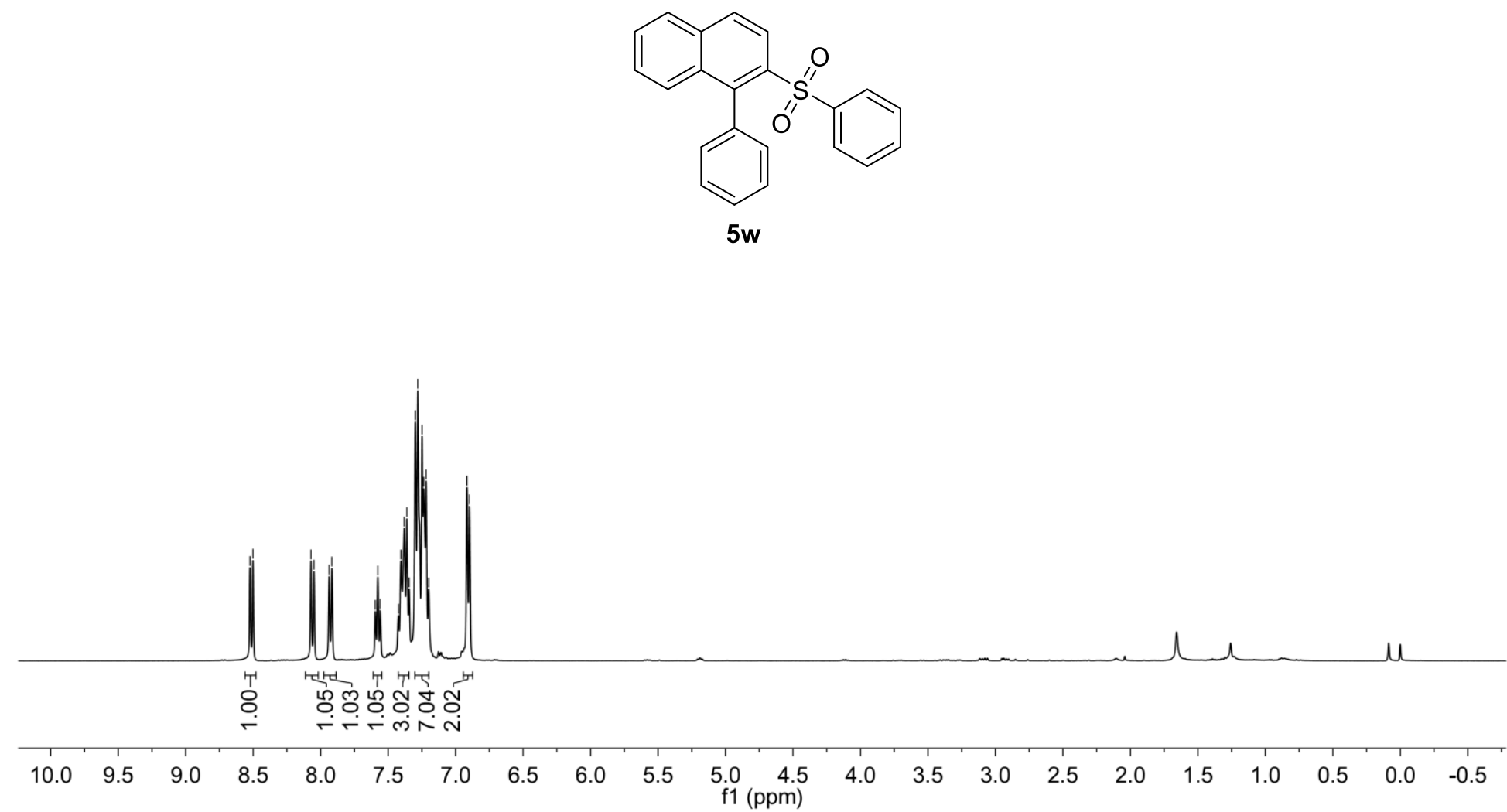

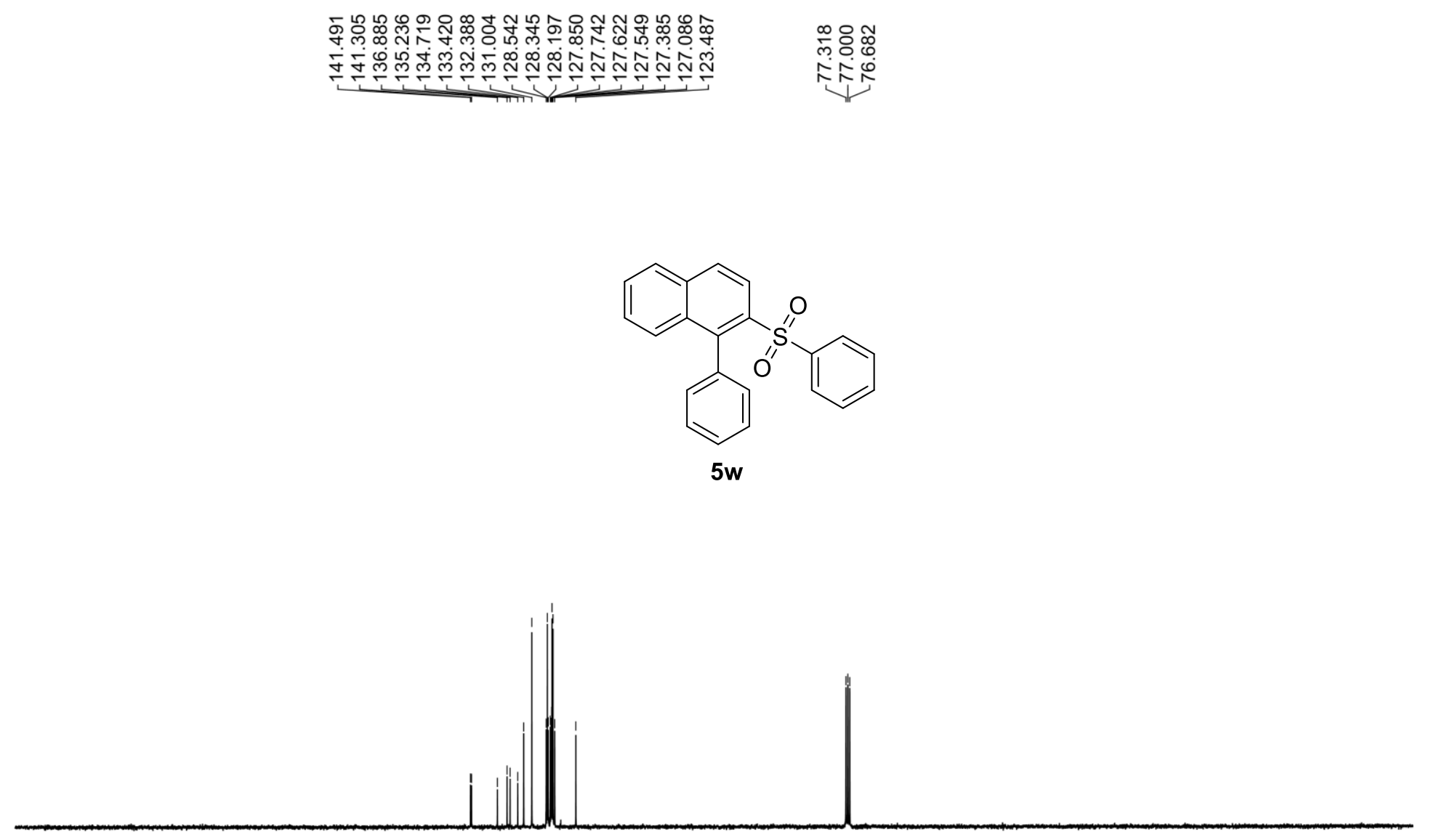

$\begin{array}{llllllllllllllllllllll}210 & 200 & 190 & 180 & 170 & 160 & 150 & 140 & 130 & 120 & 110 \begin{array}{l}100 \\ \mathrm{f} 1(\mathrm{ppm})\end{array} & 90 & 80 & 70 & 60 & 50 & 40 & 30 & 20 & 10 & 0 & -10\end{array}$ 


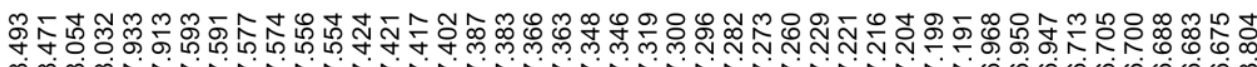

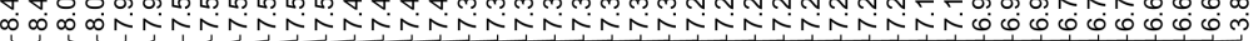
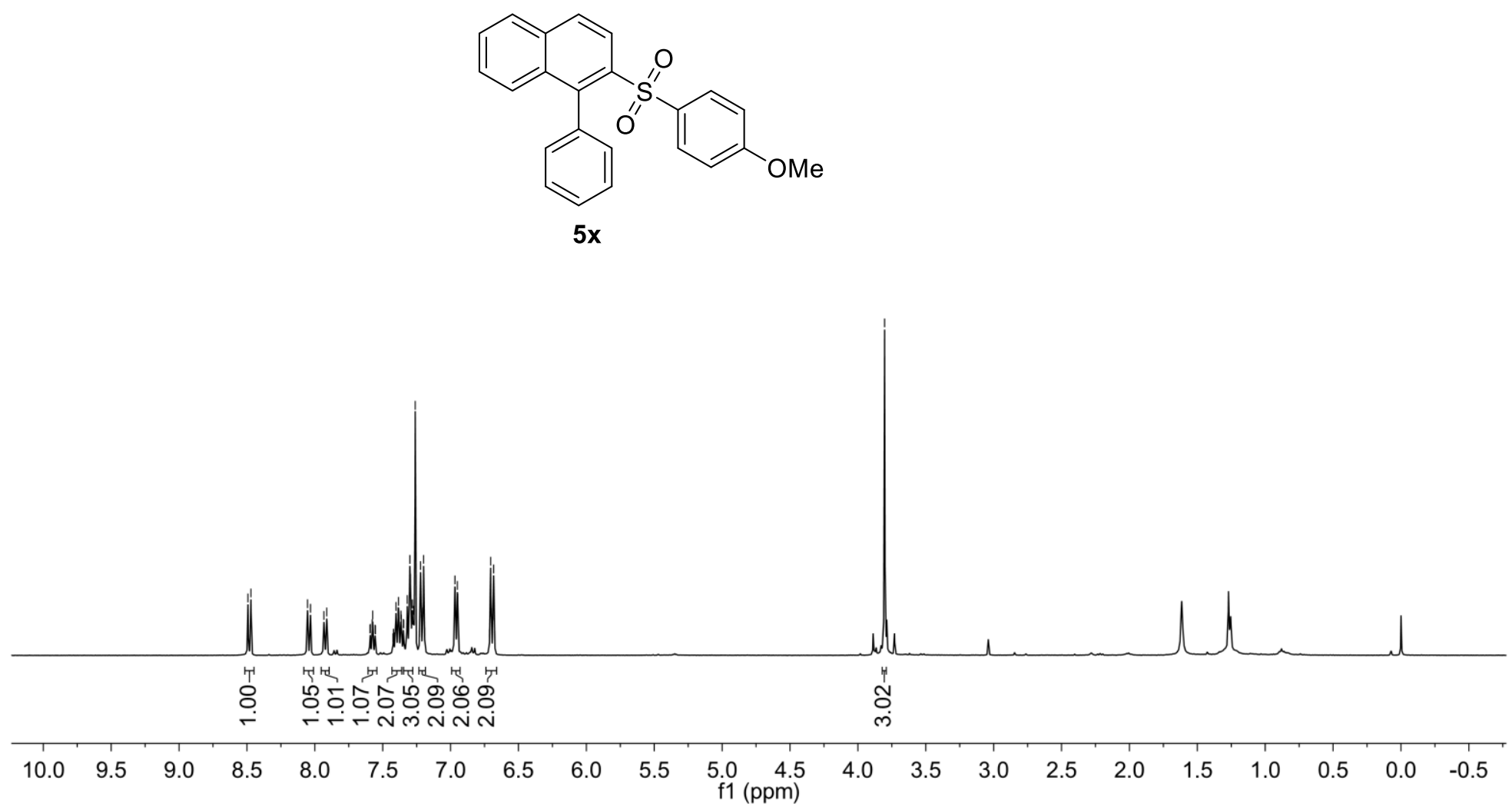


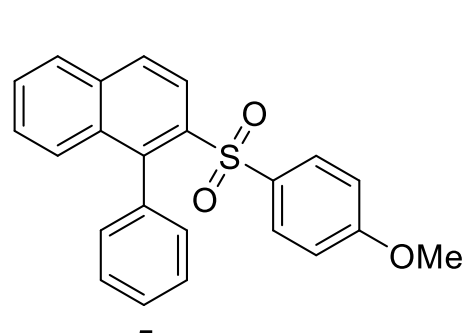

$5 \mathrm{x}$

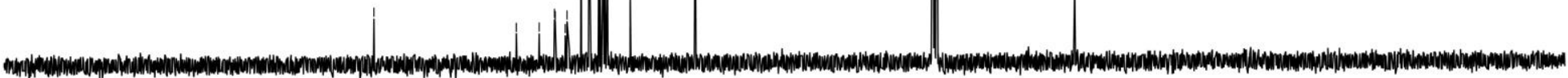

$\begin{array}{llllllllllllllllllllll}210 & 200 & 190 & 180 & 170 & 160 & 150 & 140 & 130 & 120 & 110 \begin{array}{c}100 \\ \mathrm{f} 1(\mathrm{ppm})\end{array} & 90 & 80 & 70 & 60 & 50 & 40 & 30 & 20 & 10 & 0 & -10\end{array}$ 


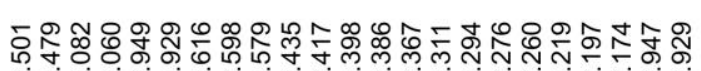

药
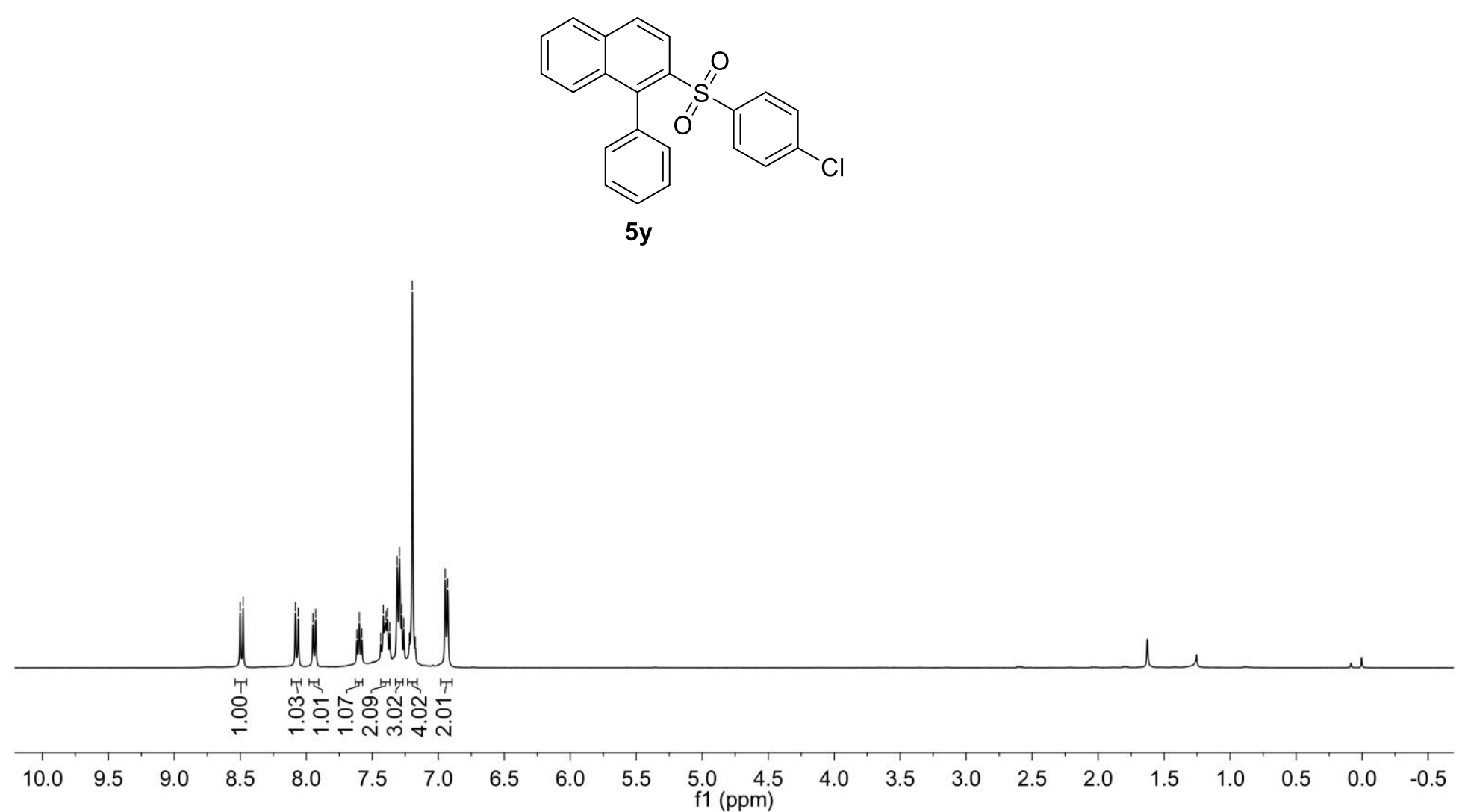

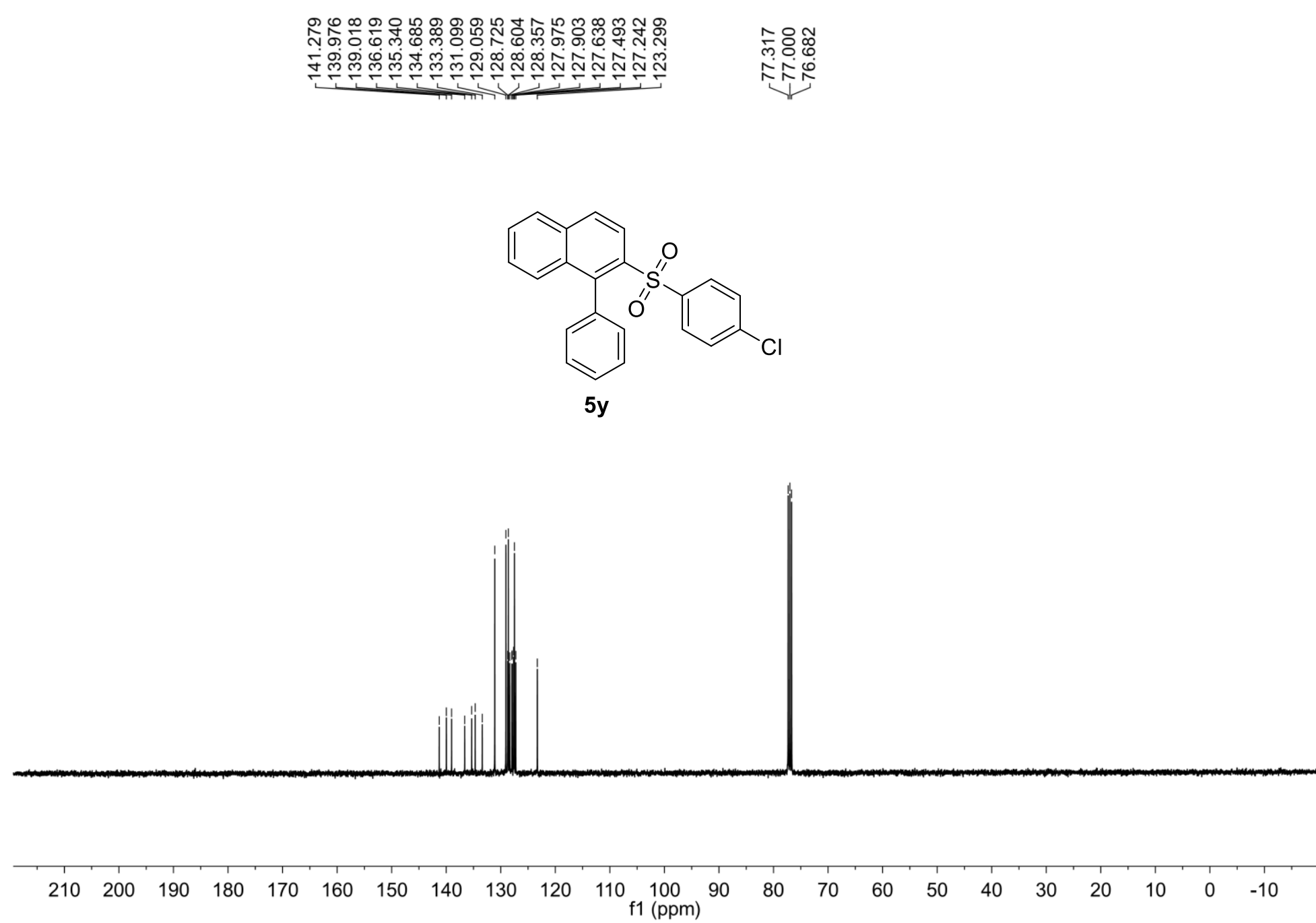


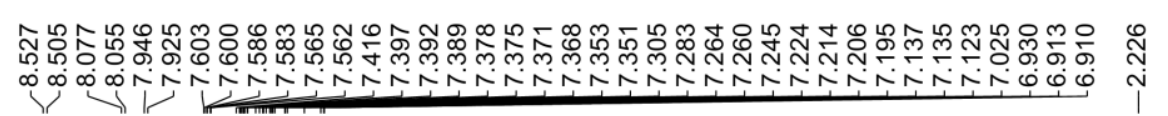

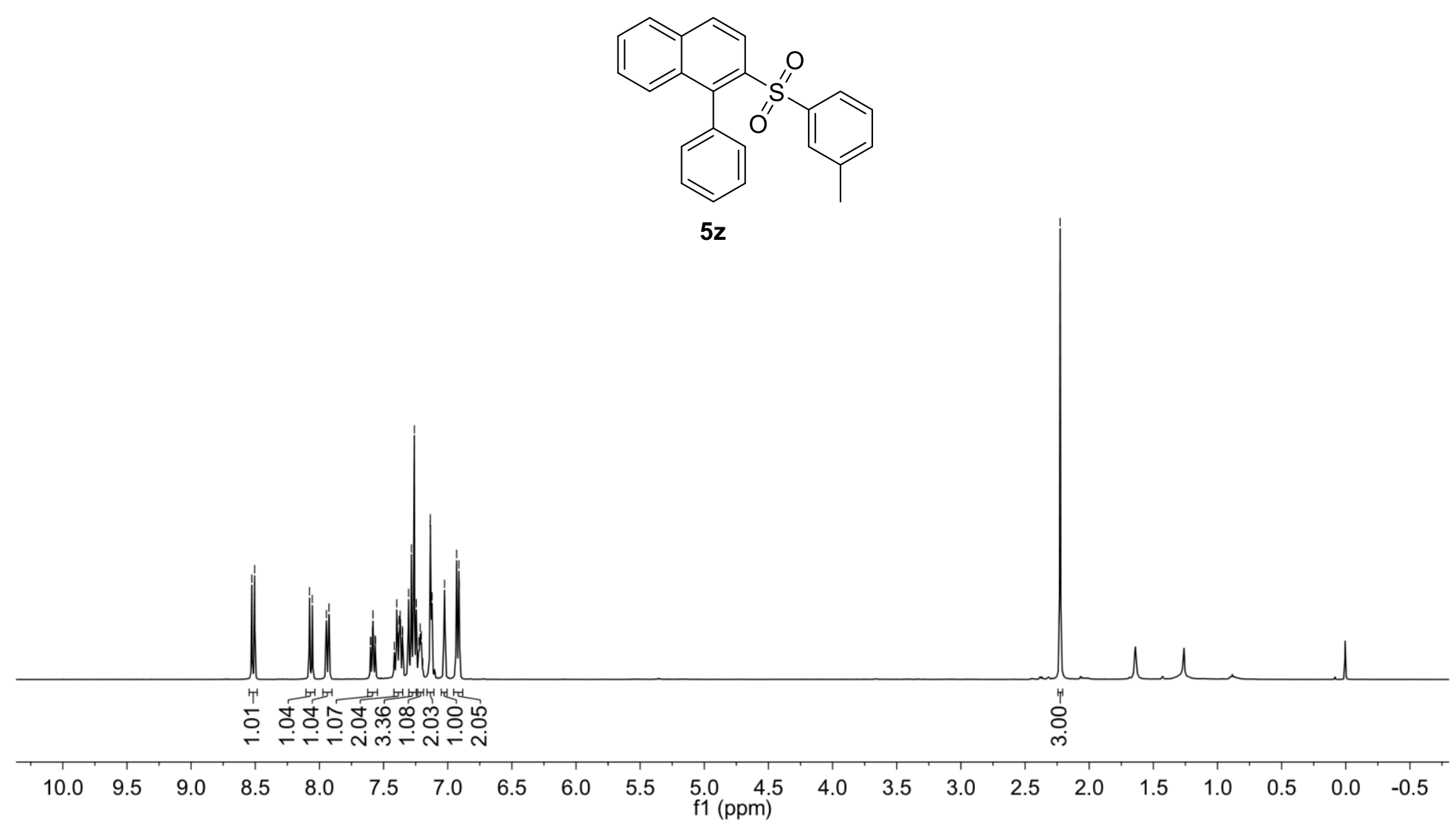




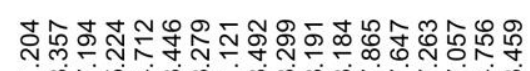

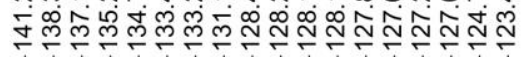

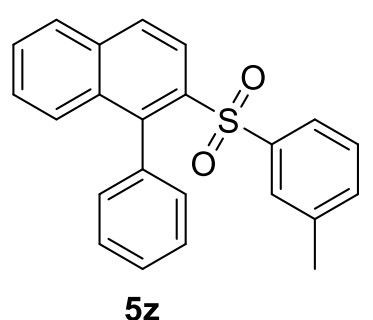

$5 z$

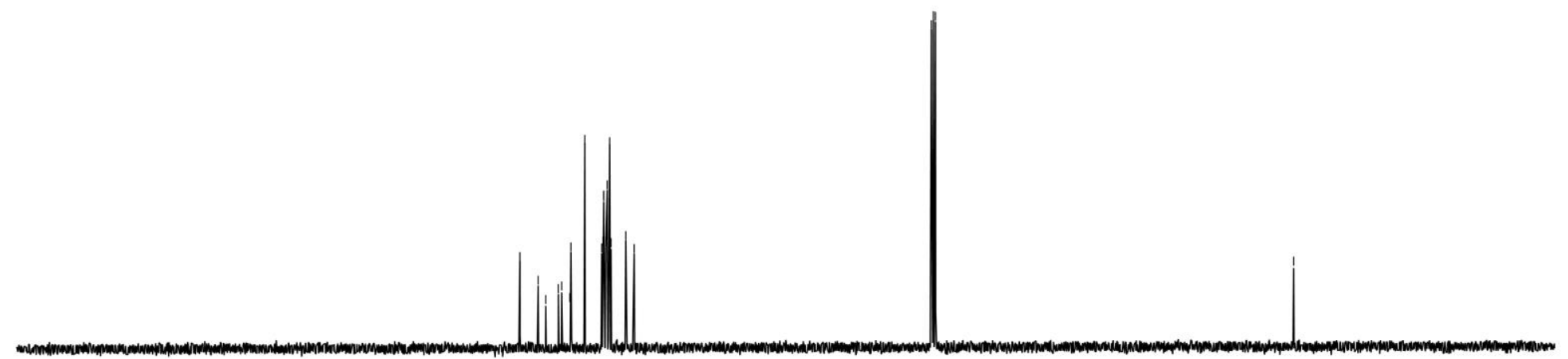

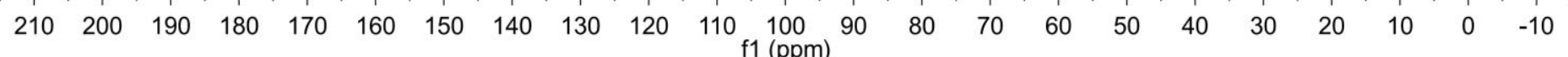




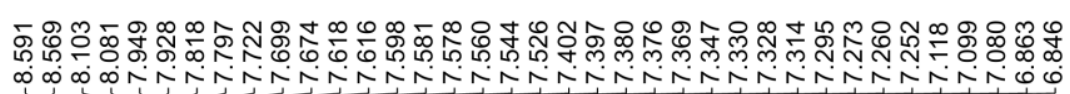
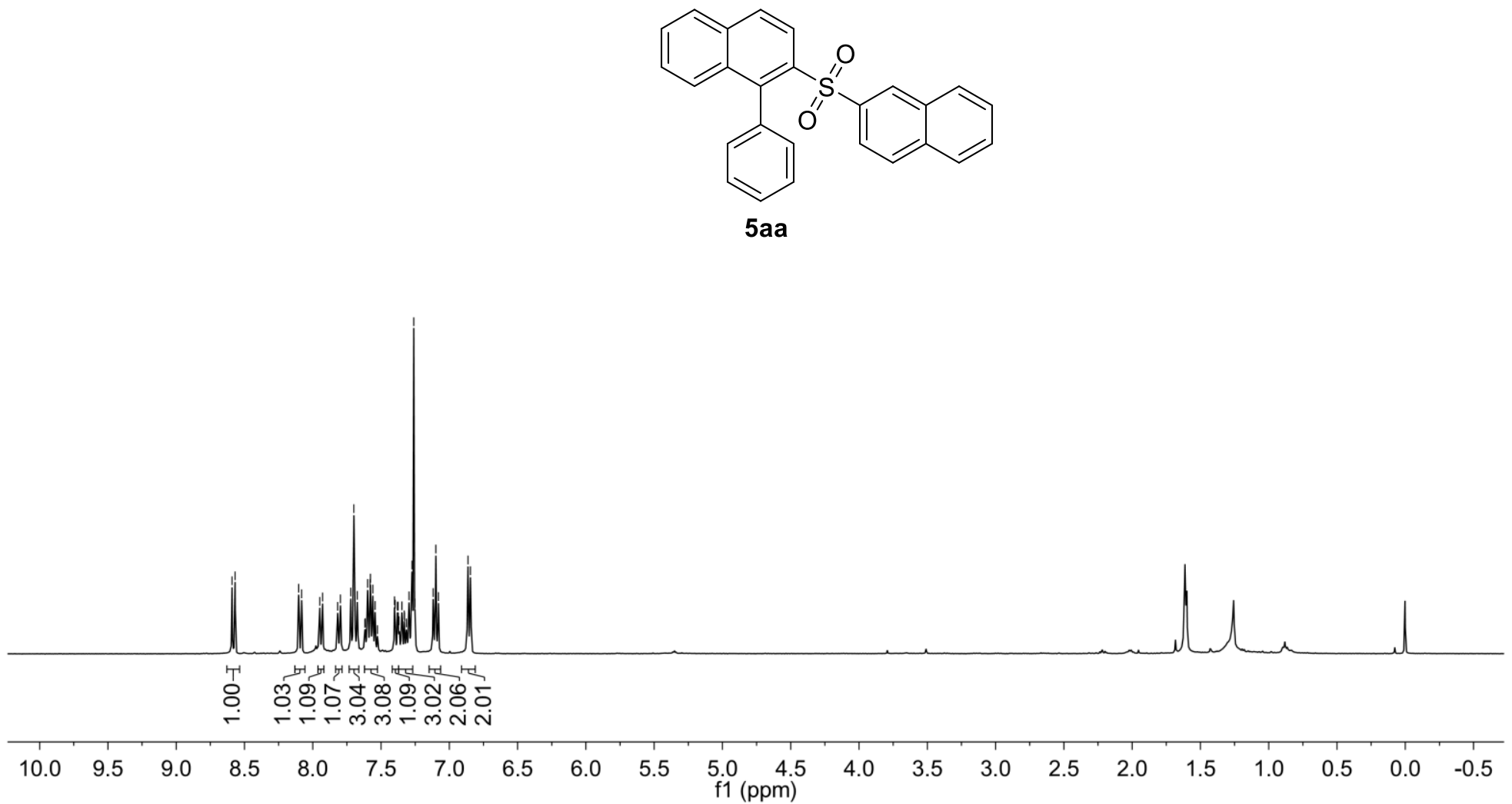


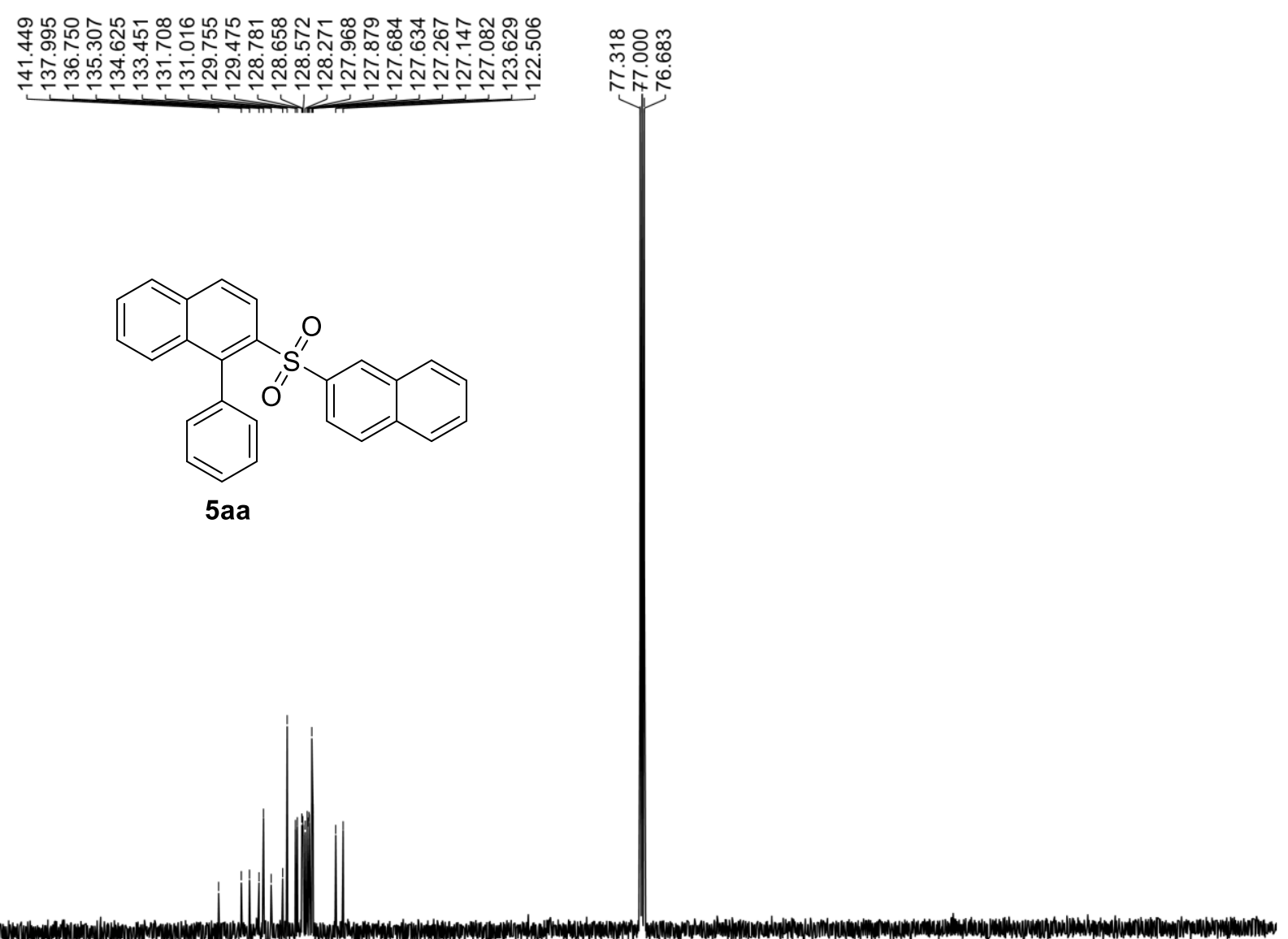

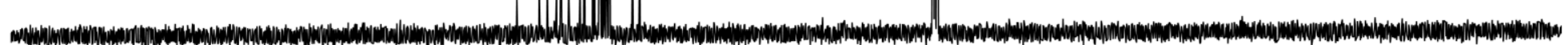

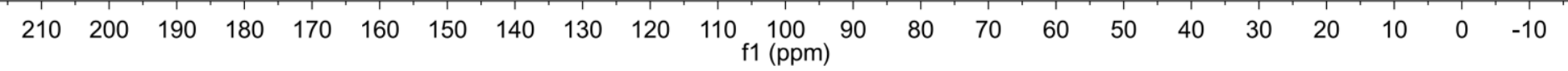

